\title{
Monitoring and modulating neuropsychiatric symptoms
}

Citation for published version (APA):

Mulders, A. E. P. (2020). Monitoring and modulating neuropsychiatric symptoms: in Parkinson's disease and obsessive-compulsive disorder. [Doctoral Thesis, Maastricht University]. Maastricht University. https://doi.org/10.26481/dis.20201105am

Document status and date:

Published: 01/01/2020

DOI:

10.26481/dis.20201105am

Document Version:

Publisher's PDF, also known as Version of record

\section{Please check the document version of this publication:}

- A submitted manuscript is the version of the article upon submission and before peer-review. There can be important differences between the submitted version and the official published version of record.

People interested in the research are advised to contact the author for the final version of the publication, or visit the DOI to the publisher's website.

- The final author version and the galley proof are versions of the publication after peer review.

- The final published version features the final layout of the paper including the volume, issue and page numbers.

Link to publication

\footnotetext{
General rights rights.

- You may freely distribute the URL identifying the publication in the public portal. please follow below link for the End User Agreement:

www.umlib.nl/taverne-license

Take down policy

If you believe that this document breaches copyright please contact us at:

repository@maastrichtuniversity.nl

providing details and we will investigate your claim.
}

Copyright and moral rights for the publications made accessible in the public portal are retained by the authors and/or other copyright owners and it is a condition of accessing publications that users recognise and abide by the legal requirements associated with these

- Users may download and print one copy of any publication from the public portal for the purpose of private study or research.

- You may not further distribute the material or use it for any profit-making activity or commercial gain

If the publication is distributed under the terms of Article $25 \mathrm{fa}$ of the Dutch Copyright Act, indicated by the "Taverne" license above, 
MONITORING AND MODULATING NEUROPSYCHIATRIC SYMPTOMS

In Parkinson's disease and obsessive-compulsive disorder

Anne Mulders 
(C) Anne E.P. Mulders, Maastricht 2020

Cover art: "Vast" by Emily Magone

www.emilymagone.com

Layout: $\quad$ Anne Mulders

Printed by: $\quad$ Ridderprint BV, Ridderkerk, the Netherlands

ISBN: $\quad$ 978-94-6380-956-6

All rights reserved. No part of this book may be reproduced or transmitted in any form or by any means, without prior permission in writing by the author, or when appropriate, by the publishers of the publication. 


\title{
MONITORING AND MODULATING NEUROPSYCHIATRIC SYMPTOMS
}

In Parkinson's disease and obsessive-compulsive disorder

\author{
PROEFSCHRIFT \\ ter verkrijging van de graad van doctor aan de Universiteit Maastricht, \\ op gezag van de Rector Magnificus, Prof.dr. Rianne M. Letschert \\ volgens het besluit van het College van Decanen, \\ in het openbaar te verdedigen op donderdag \\ 5 november 2020 om 12.00 uur
}

door

Anne Evangelia Philomena Mulders

Geboren op 22 december 1986 


\section{Promotores}

Prof. dr. A.F.G. Leentjens

Prof. dr. Y. Temel

\section{Copromotor}

Dr. A.H.J. Moonen

\section{Beoordelingscommissie}

Prof. dr. F.R.J. Verhey (voorzitter)

Prof. dr. B.R. Bloem (Radboudumc)

Prof. dr. S.A. Kotz

Dr. S. Rutten (Amsterdam UMC)

Dr. A.Y.J.M. Smeets

The research described in this thesis was performed at the School for Mental Health and Neuroscience, Department of Psychiatry and Neuropsychology and at the Department of Neurosurgery, Maastricht University, Maastricht, the Netherlands.

De Hersenstichting sponsored the research described in Chapter 4 and the Michael J. Fox Foundation for Parkinson's research sponsored the research presented in Chapters 8 and 9. Printing of this thesis was kindly supported by de Parkinson Vereniging.
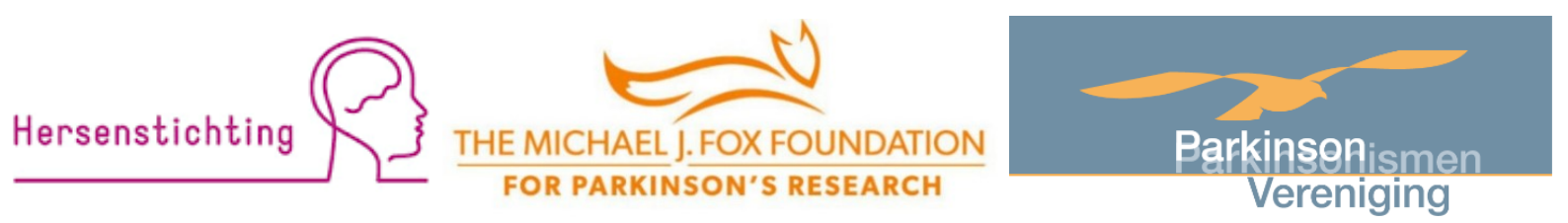


\section{"You can't stop the waves, but you can learn to surf", \\ Jon Kabat-Zinn}

To all (mental) health-care workers who continuously make an effort to improve the quality of life of patients 



\section{Contents}

$\begin{array}{lll}\text { Chapter } 1 \text { General introduction } & 9\end{array}$

Chapter 2 Deep brain stimulation of the subthalamic nucleus in 27 obsessive-compulsive disorder: neuroanatomical and pathophysiological considerations European Neuropsychopharmacology, 2016

Chapter 3 Choreatic side-effects of the anteromedial subthalamic nucleus in treatment-resistant obsessive-compulsive disorder World Neurosurgery, 2017

Chapter 4 Tryptophan depletion in PD patients treated with STN-DBS: effects on mood and behaviour

Chapter 5 The association between surgical characteristics and cognitive decline following deep brain stimulation of the subthalamic nucleus in Parkinson's disease

Submitted

Chapter 6 Network analysis of symptoms in a Parkinson patient using experience sampling data: $A n n=1$ study Movement Disorders, 2018

Chapter 7 Usability of the experience sampling method in Parkinson's disease on a group and individual level

Movement Disorders, 2020

Chapter 8 Cognitive behavioural therapy for anxiety disorders in Parkinson's disease: Design of a randomised controlled trial to assess clinical effectiveness and changes in cerebral connectivity

Journal of Psychosomatic Research, 2018

Chapter 9 Cognitive behavioural therapy for anxiety disorders in 183 Parkinson's disease: development and content of a specialised and tailored treatment programme Submitted

Chapter 10 General discussion

Summary

Samenvatting

Knowledge Valorisation

Dankwoord

Curriculum Vitae

Publications

Thesis defenses from MHeNS 




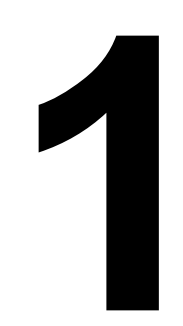

General Introduction 


\section{Neuropsychiatric symptoms and the basal ganglia}

Neuropsychiatric symptoms include a broad range of psychiatric symptoms that are believed to be mediated by alterations in brain functions and responsive to specific pharmacotherapeutic and psychotherapeutic interventions ${ }^{1-3}$. Common neuropsychiatric symptoms include depressive mood, euphoria, agitation and aggression, irritability or lability, anxiety, delusions and hallucinations, changes in eating behaviour, sleep disturbance and night-time behavioural disturbances, disinhibition, and stereotyped behaviours ${ }^{1}$. Neuropsychiatric symptoms frequently occur in neurological and neurodegenerative diseases, such as Parkinson's disease (PD), cerebrovascular disease, Alzheimer's disease and other dementias ${ }^{1}$. Moreover, certain conditions that were once considered to be manifestations of psychodynamic conflict or a strictly environmental disease, are now considered neuropsychiatric disorders, such as obsessive-compulsive disorder (OCD) ${ }^{2}$ and schizophrenia ${ }^{4}$.

Although neuropsychiatric symptoms and neuropsychiatric disorders are assumed to reflect changes in brain function, their exact underlying biological mechanisms remain largely unknown. Characterising obvious neuropathology in relation to neuropsychiatric symptoms is complicated by the large overlap of neuropsychiatric symptoms across disorders and diseases with different underlying pathologies ${ }^{1}$. It has been suggested that, regardless of the underlying pathology, neuropsychiatric symptoms are associated with disruption of cortical-basal ganglia-thalamocortical brain circuits that lead to similar symptoms across different diseases ${ }^{1,5}$.

The basal ganglia (BG) are a group of interconnected sub-cortical nuclei located at the diencephalic-mesencephalic areas. The BG consists of the caudate nucleus, putamen, pallidal complex, nucleus accumbens, substantia nigra (pars compacta and reticulate) and the subthalamc nucleus (STN) ${ }^{6}$. While in the past the basal ganglia were believed to play a role in motor functioning only, it has been recognised that these structures also play an important role in emotional, behavioural and cognitive functions ${ }^{7}$. The cortico-basal ganglia-thalamocortical circuits (or loops) originate from specific cortical areas, project to the striatum (caudate nucleus, putamen and ventral striatum), connect to the globus pallidus and substantia nigra, then to the thalamus, and finally back to the frontal cortex. While the anatomical structures are the same for all loops, they are believed to be partially segregated and integrated ${ }^{8}$ : the dorsolateral frontal cortex 
projects to the dorsolateral part of the caudate nucleus, the anterior cingulate cortex (ACC) projects to the medial striatal/nucleus accumbens area, and the orbitofrontal cortex (OFC) projects to the ventral striatal areas ${ }^{6,7,9}$. In addition, there are projections to and from other cortical and subcortical structures related to each circuit, resulting in a direct, indirect and hyperdirect pathway within each circuit ${ }^{10-12}$. Two disorders that are related to pathology of the frontal-basal ganglia circuitry are PD and $O C D^{13}$.

\section{Parkinson's disease}

Parkinson's disease (PD) is a common movement disorder and the second most prevalent neurodegenerative disorder after Alzheimer's disease. The estimated prevalence of $P D$ is $1-2 \%$ of the population of 65 years and older and increases with age $^{14,15}$. According to the Queens Square Brain Bank criteria, PD is defined by the presence of bradykinesia, together with at least one of the following motor symptoms: rigidity, resting tremor, or postural instability, and the absence of atypical features such as early severe autonomic disturbances, cerebellar or pyramidal features or lack of response to dopaminergic treatment ${ }^{16,}{ }^{17}$. Recently, new clinical criteria for PD diagnosis have been published on behalf of the International PD and Movement Disorders Society (the MDS-PD criteria) ${ }^{18}$. In the MDS-PD criteria, bradykinesia must occur in combination with resting tremor, rigidity or both. However, postural changes are left out. In addition, the MDS-PD criteria define supportive criteria, absolute exclusion criteria and red flags. At least two supportive criteria, the absence of absolute exclusion criteria, and no red flags are required for a diagnosis of "clinically established PD" (see Table 1).

The clinical motor symptoms of PD are caused by progressive loss of neurons in the substantia nigra pars compacta ( $\mathrm{SNpc}$ ), accompanied by the formation of Lewy neurites and intraneuronal Lewy bodies ${ }^{19}$. According to the staging procedure of PD pathology as proposed by Braak and colleagues (2004), a premotor period in which typical pathological changes, Lewy neuritis and Lewy bodies spread from the olfactory bulb and vagus nerve to lower brainstem regions (stages 1 and 2), is followed by a symptomatic period in which pathological changes involve the midbrain including the substantia nigra (stage 3), the mesocortex (stage 4) and eventually the neocortex (stage 5 and 6$)^{20}$. 
Table 1. MDS clinical diagnostic criteria for Parkinson's disease ${ }^{18}$

\begin{tabular}{|c|c|c|}
\hline Supportive criteria & $\begin{array}{c}\text { Absolute exclusion } \\
\text { criteria }\end{array}$ & Red flags \\
\hline 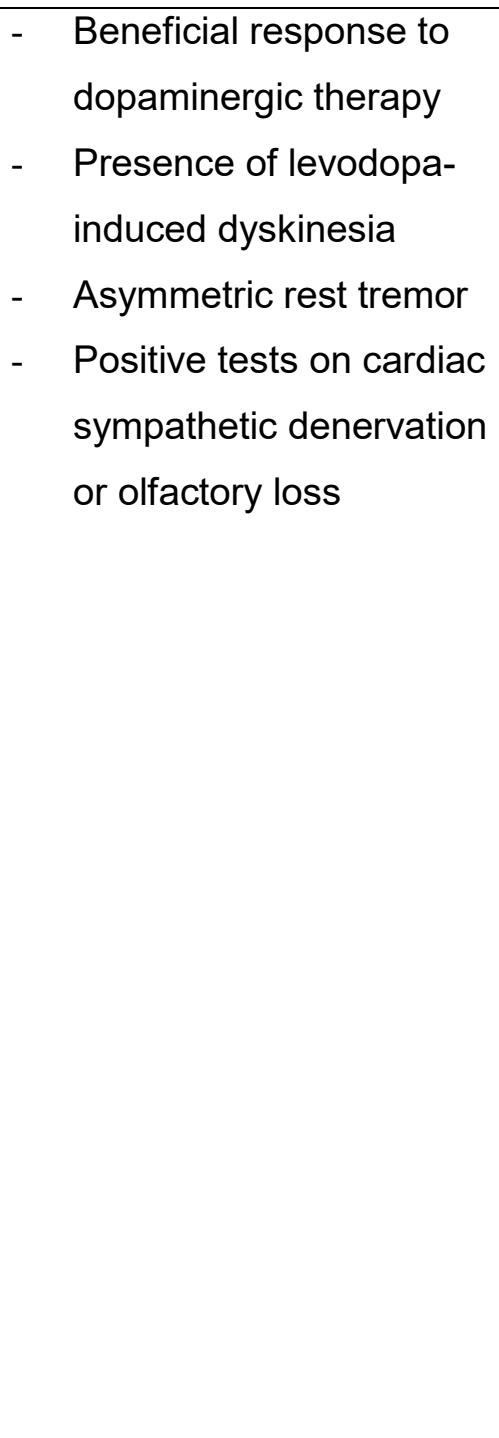 & $\begin{array}{l}\text { - } \begin{array}{l}\text { Cerebellar abnormalities } \\
\text { - }\end{array} \text { Frontotemporal } \\
\text { cognitive changes or } \\
\text { primary progressive } \\
\text { aphasia } \\
\text { - } \quad \text { Supra nuclear gaze } \\
\text { palsy } \\
\text { - Parkinsonian features } \\
\text { restricted to the lower } \\
\text { limbs for more than } 3 \\
\text { years } \\
\text { Use of anti- } \\
\text { dopaminergic therapy } \\
\text { Absence of levodopa } \\
\text { response } \\
\text { Cortical findings (e.g. } \\
\text { apraxia) }\end{array}$ & 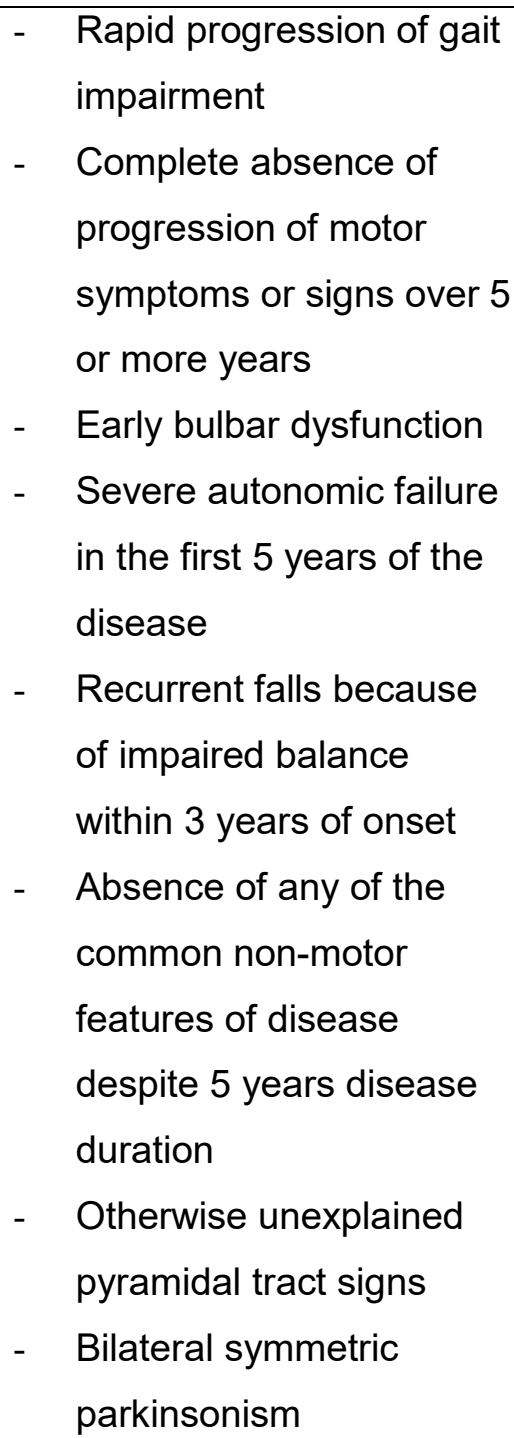 \\
\hline
\end{tabular}

PD is incurable and therapy is aimed at controlling symptoms by replacing the depleted dopamine or by enhancing dopaminergic functioning with antiparkinsonian medication. Most patients respond well to Levodopa ${ }^{21}$, and is therefore considered the gold standard pharmacotherapy for PD patients. However, as the disease progresses, motor fluctuations and disabling dyskinesia develop in approximately $50 \%$ of patients, usually starting with 'end-of dose wearing-off', but eventually leading to unpredictable and disabling fluctuations between on and off state ${ }^{22}$. Although the concomitant use of other neurochemical agents such as monoamine oxidase B inhibitors, dopaminergic agonists, anticholinergic agents, and amantadine may offset the diminished efficacy of levodopa, these agents eventually lose their efficacy and are associated with 
significant side-effects ${ }^{23}$. For patients who are affected by long-term complications of Levodopa therapy, deep brain stimulation (DBS) is increasingly accepted as an adjunct therapy ${ }^{24}$. DBS, which will be discussed in more detail later, is a surgical intervention that is aimed at restoring the balance of cortical-basal ganglia-thalamocortical brain circuits that are disrupted in $P D^{25}$.

\section{Neuropsychiatric symptoms in Parkinson's disease}

Apart from motor symptoms, PD is characterised by a variety of non-motor symptoms that contribute significantly to disability, reduced quality of life as well as caregiver distress $^{26-30}$. Common non-motor symptoms in PD include olfactory loss, sleep disorders, autonomic dysfunction, cognitive alterations, and neuropsychiatric symptoms, including depression, anxiety, apathy and hallucinations ${ }^{30,31}$. This thesis will mainly focus on cognitive and emotional disturbances in PD. Cognitive deficits in PD increase with age, disease-duration, and severity of PD, and commonly present as a dysexecutive syndrome with difficulties in abstract thinking, cognitive flexibility, decision-making and planning ${ }^{32}$. There is substantial heterogeneity in the clinical presentation and progression of cognitive impairments in PD, which likely reflects the diversity of brain damage caused by the disease ${ }^{32}$.

The most prevalent mood symptoms in PD are depression (20-40\%) and anxiety (35$55 \%)^{33}, 34$. Anxiety can have a negative impact on the motor symptoms of PD, including freezing, gait, dyskinesias and on-off fluctuations ${ }^{35}$, and depression has been found to, if untreated, result in greater functional disability and faster physical and cognitive deterioration $^{36}$. Due to the high overlap of symptoms of anxiety and depression on one hand, and other core symptoms of PD, such as sleep disturbances and fatigue, attentional and concentration changes, loss of appetite or weight changes on the other hand, mood symptoms in PD are often underdiagnosed and undertreated ${ }^{37}$. Moreover, recognising symptoms of anxiety and depression is further complicated by the fact that these symptoms, similar to motor symptoms, can be off-period related and fluctuate over the course of the day ${ }^{38,39}$, which makes it difficult to capture them with routine cross-sectional assessments that are usually retrospective in nature. Recently, it has been suggested that the experience sampling method (ESM), a digital diary tool that captures moment-to-moment changes in physical and mental states, as well as 
contextual factors, could be used to elucidate relationships between motor and mood states in PD patients ${ }^{40}$.

Mood symptoms often occur before the onset of motor symptoms in PD and can be responsive to antiparkinsonian medication in some patients. Therefore, it has been suggested that these symptoms reflect underlying disease-pathology ${ }^{38}$. While there is considerable evidence that PD is the result of abnormal activity in the frontal basalganglia 'motor' circuit due to loss of dopamine ${ }^{5}$, the frontal basal-ganglia 'limbic' circuit, which is involved in the regulation of emotional behaviour and includes the amygdala, ventral striatum, ventral ACC and OFC, is also highly dependent on dopaminergic neurotransmission ${ }^{41}$. However, symptoms do not always improve following dopaminergic replacement therapy, and studies have suggested that the noradrenergic and serotonergic neurotransmitter systems are also involved ${ }^{42-45}$. On the other hand, it has been suggested that fears and social implications associated with suffering from a neurodegenerative disorder or from the motor symptoms of PD can provoke or exacerbate mood symptoms in $\mathrm{PD}^{46}$. Indeed, common anxiety symptoms in PD include worry and insecurity of having PD, worry relating to motor symptoms, such as fear of falling, fear of further physical deterioration, but also worry relating to social embarrassment and social withdrawal due to motor symptoms and off-periods ${ }^{47}$. In addition, major life-events seem to play an important role for the onset of mood symptoms in PD, which seems to be modulated by social support and coping capacities $^{48}$. Given the complex interaction between physiological and psychological factors, the diversity of the disease, as well as the fact that a number of mood symptoms relate to PD symptomatology, interventions should be tailored to the specific needs and circumstances of PD patients.

To date there are few evidence-based treatments for depression in PD. There have been mixed reviews on the efficacy of antidepressants for depression in patients with PD. There is increasing evidence that treatment with selective serotonin reuptake inhibitors (SSRIs) or serotonin-norepinephrine reuptake inhibitors (SNRIs) are beneficial in alleviating the symptoms of depression in $\mathrm{PD}^{49}$. However, several metaanalyses have reported that tricyclic antidepressants are more effective compared to SSRIs or SNRIs ${ }^{50}$. Recently, cognitive behavioural therapy (CBT) has been proven to be effective for depression in $\mathrm{PD}^{51,52}$. CBT is a psychotherapeutic intervention that 
aims to change maladaptive patterns of thinking or behaviour. Evidence from neuroimaging studies suggest that CBT is able to alter activity and connectivity in several brain regions believed to be involved in mood symptoms ${ }^{53,54}$.

For anxiety in PD, no evidence-based treatment is available at present and it has been reported that up to $57 \%$ of $\mathrm{PD}$ patients with anxiety are untreated ${ }^{55}$. While pharmacological treatment, such as antidepressants or benzodiazepines are the most applied treatment by practitioners, one study reported that of the PD patients who received pharmacotherapy, $83 \%$ still experienced a current anxiety disorder ${ }^{55}$. Moreover, antidepressants often involve undesired side-effects, and benzodiazepines are associated with increased risk of falling ${ }^{35,56}$. These findings suggests that anxiety in PD is difficult to treat and there is an urgent need for the development of evidencebased treatment approaches tailored to address PD-specific anxiety symptomatology.

\section{Obsessive-compulsive disorder}

OCD is a neuropsychiatric disorder characterised by chronic intrusive and distressing thoughts or impulses (obsessions) and repetitive and ritualistic thoughts or mental acts (compulsions). OCD has a significant negative impact on multiple domains of quality of life ${ }^{57}$. The estimated lifetime prevalence of OCD is $1-3 \% 57-59$. Previously, OCD was grouped with "anxiety disorders" in the fourth edition of the Diagnostic and Statistical Manual for Mental Disorders (DSM-IV). However, findings from phenotypic, neuroimaging, genetic and treatment studies suggested that OCD is both phenomenologically and behaviourally distinct from other anxiety disorders, and was therefore placed in a new category called "obsessive-compulsive and related disorders' in the DSM-5 57, 60, 61. In this new classification, four clusters have been identified: symmetry/ordering, hoarding, contamination/cleaning, and obsessions/ checking. These heterogeneous symptoms have in common that they all feature repetitive thought and action, expressed in relation to external and internal stimuli58.

Recent evidence from neuroimaging studies suggest that OCD and OC-spectrum disorders are related to abnormalities in frontal-basal ganglia circuitry ${ }^{58}$. Especially the 'limbic' circuit seems to be implicated in the development of OCD. Functional magnetic resonance (FMRI) studies found altered activities in ACC, OFC and striatum during resting-state and during symptom expression ${ }^{62}, 63$. Moreover, altered functional connectivity between the striatum and PFC areas have been reported in OCD 
patients ${ }^{64-66}$. More evidence for the involvement of the frontal-basal ganglia circuits in OCD came from studies showing elevated rates of OC symptoms in $P D^{67-69}$. First line treatments for $O C D$ involve psychotherapeutic and/or pharmacological interventions. $C B T$ is the most effective evidence-based psychotherapeutic intervention for $O C D^{70,71}$, while SSRIs are the first choice medication for treating $O C D^{72}$. These are often used together in clinical practice ${ }^{73}$. About $60-80 \%$ of patients respond to first-line treatments, and approximately $90 \%$ to treatment including antipsychotics ${ }^{74}$. The remaining $10 \%$ of patients remain unresponsive to all therapies and suffer from treatment-refractory OCD. In recent years, DBS has become a potential treatment modality for treatmentrefractory OCD.

\section{Deep brain stimulation in Parkinson's disease and obsessive-compulsive disorder}

DBS is a neuromodulation procedure that involves stereotactic implantation of electrodes in deep targets in the brain, which are connected to a pacemaker delivering chronic and high frequency electrical stimulation and thereby modulate neural tissue at the target structure as well as surrounding and connected regions ${ }^{24}$. Although DBS is an invasive neurosurgical procedure, it has the advantage of being reversible. Furthermore, because of its adjustable stimulation parameters, it can be tailored for individual patients.

One of the most common targets for DBS in PD is the STN. The STN is a small lenticular shaped nucleus located in the diencephalon-mesencephalic junction, rostral to the substantia nigra. It distinguishes itself from all other inhibitory basal ganglia structures since it is the only glutamatergic, excitatory, nucleus ${ }^{11}$. The STN has three functional subdivisions: the dorsolateral sensorimotor region, the ventromedial associative region, and the medial tip/rostral limbic region ${ }^{11,75}$. STN-DBS has been proven effective in improving motor symptoms of PD such as tremor, rigidity and akinesia. In addition, it allows substantial reduction of dopaminergic medications and, thereby, reduces disabling dyskinesia and on-off fluctuations ${ }^{76}$. However, there have been conflicting reports on the occurrence of neuropsychiatric, behavioural and cognitive changes following STN-DBS, ranging from new onset or worsening of preexisting mood and cognitive symptoms to improvement of cognitive and neuropsychiatric symptoms. In addition, neuropsychiatric symptoms reported after 
STN-DBS range from mania and impulse control disorders to apathy and depression ${ }^{77,78}$. Changes in mood, behaviour and cognition may potentially counteract the beneficial effects of STN-DBS on the motor symptoms.

Several patient inherent risk factors have been identified that may partially explain cognitive decline following DBS, including older age, axial symptoms and prolonged levodopa dosage ${ }^{79,80}$. Furthermore, it has been suggested that stimulation and surgical factors, such as the volume of tissue activated within and outside the STN, the location of the electrode, or cortical or subcortical lesions due to the lead trajectory, are associated with changes in cognitive functioning ${ }^{81}$. However, research on the factors contributing to cognitive changes following STN-DBS have led to inconsistent findings. Similarly, the occurrence of mood symptoms following DBS, such as depression, has been associated with a number of causes including current spreading to the limbic region of the STN, post-operative withdrawal of dopaminergic medication, pre-existing depressive symptoms, and mal-adaptation to the new physical and psychosocial situation following DBS surgery ${ }^{77,82,83}$. Moreover, experimental studies showed that STN stimulation inhibited dorsal raphe nucleus neuronal firing and a reduced release of serotonin in the rat, which suggests that alteration in the serotonergic (5-HT) neurotransmitter might be involved in the neuropsychiatric sideeffects following STN-DBS ${ }^{84-86}$.

Interestingly, following the observations that STN-DBS resulted in an improvement in motor symptoms as well as obsessive and compulsive symptoms in PD patients with advanced PD and comorbid OCD, DBS gained attention as treatment option for treatment-refractory OCD patients ${ }^{87,88}$. In recent years, several clinical trials and case reports have published promising results regarding the effect of STN-DBS in OCD patients ${ }^{89}$. In addition, several other DBS targets that are part of the frontal-basal ganglia 'limbic' circuit have been studied, such as the ventral capsule/ventral striatum, the anterior limb of the internal capsule, the nucleus accumbens and the inferior thalamic peduncle ${ }^{89}$. Recent systematic reviews and meta-analyses found similar percentages of improvement in these different target areas with a reduction of $45-48 \%$ in obsessive and compulsive symptoms following DBS. Moreover, the global percentage of responders -a minimum reduction of $35 \%$ on the Yale-Brown Obsessive-Compulsive Scale- has been estimated at 58-67\% 89,90 . In light of these 
findings, many questions remain regarding the underlying mechanisms of DBS on frontal-basal ganglia circuits and condition-specific neurobiology.

\section{Aims of this thesis}

This thesis has several aims divided into two parts:

Part 1: DBS studies in $O C D$ and $P D$

The first aim was to explore the role of the STN in OCD circuitry and its pathophysiological mechanisms based on observations from DBS studies (Chapter 2 and 3). The second aim was to study underlying mechanisms of emotional and cognitive side-effects of STN-DBS in PD patients (Chapter 4 and 5).

Part 2: Mood symptoms in PD

The third aim of this thesis was to explore the usability of ESM in PD at the individual and population level by exploring associations between motor and mood symptoms in patients who suffer from motor fluctuations (Chapter 6 and 7). And finally, we aimed to develop a treatment programme for anxiety in PD, which is supported by the knowledge of the underlying aetiology and correlations of anxiety in PD (Chapter 8 and 9).

\section{Outline of this thesis}

Part 1

DBS in OCD Chapter 2 provides a narrative review of the functional role of the STN in the pathophysiology of OCD, in which we aimed to integrate neuroanatomical and pathophysiological findings from DBS studies.

Chapter 3 describes a patient who received anteromedial STN-DBS for treatment-resistant OCD, but developed motor side-effects that interfered with DBS programming.

DBS in PD In Chapter 4 we aimed to investigate the role of 5-HT on mood and behaviour in PD patients with STN-DBS by temporarily reducing the level of available $5-\mathrm{HT}$ in the brain by means of tryptophan depletion. 
In Chapter $\mathbf{5}$ we aimed to study whether cognitive decline following STN-DBS in PD was associated with surgical characteristics.

Part 2

ESM in PD In Chapter 6 we aimed to study longitudinal associations between motor symptoms and mood symptoms in a PD patient by using ESM data. In Chapter 7 we explored the usability and sensitivity of routinely collected ESM data in PD patients on a group and individual level.

CBT for PD Chapter 8 describes the rationale and methodology of a randomised controlled trial that aims to study the clinical effectiveness, alterations in brain circuitry, and cost-effectiveness of a specialised CBT programme for anxiety in PD. Chapter 9 presents the development, specific structure and content of the specialised treatment programme we developed for anxiety in PD, which is based on CBT principles.

Summary Chapter $\mathbf{1 0}$ provides a general discussion, including a summary of the main findings of the various chapters, and implications and recommendations for future research and clinical practice. 


\section{REFERENCES}

1. Husain M. Transdiagnostic neurology: neuropsychiatric symptoms in neurodegenerative diseases. Brain 2017;140(6):1535-1536.

2. Stein DJ. Obsessive-compulsive disorder. Lancet 2002;360(9330):397-405.

3. Taber KH, Hurley RA, Yudofsky SC. Diagnosis and treatment of neuropsychiatric disorders. Annu Rev Med 2010;61:121-133.

4. Price BH, Adams RD, Coyle JT. Neurology and psychiatry: closing the great divide. Neurology 2000;54(1):8-14.

5. DeLong MR, Wichmann T. Circuits and circuit disorders of the basal ganglia. Arch NeurolChicago 2007;64(1):20-24.

6. Alexander GE, DeLong MR, Strick PL. Parallel organization of functionally segregated circuits linking basal ganglia and cortex. Annu Rev Neurosci 1986;9:357-381.

7. Tekin S, Cummings JL. Frontal-subcortical neuronal circuits and clinical neuropsychiatry - An update. J Psychosom Res 2002;53(2):647-654.

8. Haynes WI, Haber SN. The organization of prefrontal-subthalamic inputs in primates provides an anatomical substrate for both functional specificity and integration: implications for Basal Ganglia models and deep brain stimulation. J Neurosci 2013;33(11):4804-4814.

9. Alexander GE, Crutcher MD, DeLong MR. Basal ganglia-thalamocortical circuits: parallel substrates for motor, oculomotor, "prefrontal" and "limbic" functions. Prog Brain Res 1990;85:119-146.

10. Alexander GE, Crutcher MD. Functional architecture of basal ganglia circuits: neural substrates of parallel processing. Trends Neurosci 1990;13(7):266-271.

11. Temel Y, Blokland A, Steinbusch HW, Visser-Vandewalle V. The functional role of the subthalamic nucleus in cognitive and limbic circuits. Prog Neurobiol 2005;76(6):393-413.

12. Nambu A, Tokuno $H$, Takada M. Functional significance of the cortico-subthalamo-pallidal 'hyperdirect' pathway. Neurosci Res 2002;43(2):111-117.

13. Ring HA, Serra-Mestres J. Neuropsychiatry of the basal ganglia. J Neurol Neurosur Ps 2002;72(1):12-21.

14. Wirdefeldt K, Adami HO, Cole P, Trichopoulos D, Mandel J. Epidemiology and etiology of Parkinson's disease: a review of the evidence. Eur J Epidemiol 2011;26 Suppl 1:S1-58.

15. Alves G, Forsaa EB, Pedersen KF, Gjerstad MD, Larsen JP. Epidemiology of Parkinson's disease. J Neurol 2008;255:18-32.

16. de Rijk MC, Rocca WA, Anderson DW, Melcon MO, Breteler MM, Maraganore DM. A population perspective on diagnostic criteria for Parkinson's disease. Neurology 1997;48(5):1277-1281.

17. Hughes AJ, Daniel SE, Kilford L, Lees AJ. Accuracy of clinical diagnosis of idiopathic Parkinson's disease: a clinico-pathological study of 100 cases. J Neurol Neurosurg Psychiatry 1992;55(3):181-184.

18. Postuma RB, Berg D, Stern M, et al. MDS clinical diagnostic criteria for Parkinson's disease. Mov Disord 2015;30(12):1591-1601.

19. Lang AE, Lozano AM. Parkinson's disease. First of two parts. N Engl J Med 1998;339(15):10441053.

20. Braak H, Ghebremedhin E, Rub U, Bratzke H, Del Tredici K. Stages in the development of Parkinson's disease-related pathology. Cell Tissue Res 2004;318(1):121-134.

21. LeWitt PA. Levodopa Therapy for Parkinson's Disease: Pharmacokinetics and Pharmacodynamics. Movement Disord 2015;30(1):64-72.

22. Ahlskog JE, Muenter MD. Frequency of levodopa-related dyskinesias and motor fluctuations as estimated from the cumulative literature. Movement Disord 2001;16(3):448-458.

23. Sharma A, Szeto K, Desilets AR. Efficacy and safety of deep brain stimulation as an adjunct to pharmacotherapy for the treatment of Parkinson disease. Ann Pharmacother 2012;46(2):248-254.

24. Benabid AL. Deep brain stimulation for Parkinson's disease. Current Opinion in Neurobiology 2003;13(6):696-706. 
25. Wichmann T, DeLong MR. Deep Brain Stimulation for Movement Disorders of Basal Ganglia Origin: Restoring Function or Functionality? Neurotherapeutics 2016;13(2):264-283.

26. Schrag A. Quality of life and depression in Parkinson's disease. J Neurol Sci 2006;248(1-2):151157.

27. Schrag A, Hovris A, Morley D, Quinn N, Jahanshahi M. Caregiver-burden in parkinson's disease is closely associated with psychiatric symptoms, falls, and disability. Parkinsonism Relat Disord 2006;12(1):35-41.

28. Weintraub D. Neuropsychiatric symptoms in Parkinson disease and dementia with Lewy bodies: what geriatric psychiatry can learn. Am J Geriatr Psychiatry 2013;21(6):497-500.

29. Weintraub D, Moberg PJ, Duda JE, Katz IR, Stern MB. Effect of psychiatric and other nonmotor symptoms on disability in Parkinson's disease. J Am Geriatr Soc 2004;52(5):784-788.

30. Weintraub D, Stern MB. Psychiatric complications in Parkinson disease. Am J Geriatr Psychiatry 2005;13(10):844-851.

31. Park A, Stacy M. Non-motor symptoms in Parkinson's disease. J Neurol 2009;256 Suppl 3:293298.

32. Dujardin $\mathrm{K}$, Leentjens $\mathrm{AF}$, Langlois $\mathrm{C}$, et al. The spectrum of cognitive disorders in Parkinson's disease: a data-driven approach. Mov Disord 2013;28(2):183-189.

33. Broen MP, Narayen NE, Kuijf ML, Dissanayaka NN, Leentjens AF. Prevalence of anxiety in Parkinson's disease: A systematic review and meta-analysis. Mov Disord 2016;31(8):1125-1133.

34. Reijnders JS, Ehrt U, Weber WE, Aarsland D, Leentjens AF. A systematic review of prevalence studies of depression in Parkinson's disease. Mov Disord 2008;23(2):183-189; quiz 313.

35. Chen JJ, Marsh L. Anxiety in Parkinson's disease: identification and management. Ther Adv Neurol Diso 2014;7(1):52-59.

36. Marsh L. Depression and Parkinson's disease: current knowledge. Curr Neurol Neurosci Rep 2013;13(12):409.

37. Shulman LM, Taback RL, Bean J, Weiner WJ. Comorbidity of the nonmotor symptoms of Parkinson's disease. Movement Disord 2001;16(3):507-510.

38. Aarsland D, Marsh L, Schrag A. Neuropsychiatric symptoms in Parkinson's disease. Mov Disord 2009;24(15):2175-2186.

39. van der Velden RMJ, Broen MPG, Kuijf ML, Leentjens AFG. Frequency of mood and anxiety fluctuations in Parkinson's disease patients with motor fluctuations: A systematic review. Mov Disord 2018;33(10):1521-1527.

40. Broen MP, Marsman VA, Kuijf ML, Van Oostenbrugge RJ, van Os J, Leentjens AF. Unraveling the Relationship between Motor Symptoms, Affective States and Contextual Factors in Parkinson's Disease: A Feasibility Study of the Experience Sampling Method. Plos One 2016;11(3):e0151195.

41. Phillips ML, Drevets WC, Rauch SL, Lane R. Neurobiology of emotion perception I: The neural basis of normal emotion perception. Biol Psychiat 2003;54(5):504-514.

42. Chaudhuri KR, Schapira AH. Non-motor symptoms of Parkinson's disease: dopaminergic pathophysiology and treatment. Lancet Neurol 2009;8(5):464-474.

43. Barone P. Neurotransmission in Parkinson's disease: beyond dopamine. Eur J Neurol 2010;17(3):364-376.

44. Remy P, Doder M, Lees A, Turjanski N, Brooks D. Depression in Parkinson's disease: loss of dopamine and noradrenaline innervation in the limbic system. Brain 2005;128:1314-1322.

45. Maillet $A$, Krack $P$, Lhommee E, et al. The prominent role of serotonergic degeneration in apathy, anxiety and depression in de novo Parkinson's disease. Brain 2016;139:2486-2502.

46. Prediger RD, Matheus FC, Schwarzbold ML, Lima MM, Vital MA. Anxiety in Parkinson's disease: a critical review of experimental and clinical studies. Neuropharmacology 2012;62(1):115-124.

47. Dissanayaka NN, O'Sullivan JD, Pachana NA, et al. Disease-specific anxiety symptomatology in Parkinson's disease. Int Psychogeriatr 2016;28(7):1153-1163.

48. Rod NH, Bordelon Y, Thompson A, Marcotte E, Ritz B. Major life events and development of major depression in Parkinson's disease patients. Eur J Neurol 2013;20(4):663-670. 
49. Bomasang-Layno E, Fadlon I, Murray AN, Himelhoch S. Antidepressive Treatments for Parkinson's Disease: A Systematic Review and Meta-Analysis. Am J Geriat Psychiat 2016;24(3):S102S103.

50. Kelberman MA, Vazey EM. New Pharmacological Approaches to Treating Non-Motor Symptoms of Parkinson's Disease. Curr Pharmacol Rep 2016;2(6):253-261.

51. Dobkin RD, Mann SL, Interian A, Gara MA, Menza M. Cognitive behavioral therapy improves diverse profiles of depressive symptoms in Parkinson's disease. Int J Geriatr Psychiatry 2019;34(5):722729.

52. Dobkin RD, Menza M, Allen LA, et al. Cognitive-behavioral therapy for depression in Parkinson's disease: a randomized, controlled trial. Am J Psychiatry 2011;168(10):1066-1074.

53. Porto PR, Oliveira L, Mari J, Volchan E, Figueira I, Ventura P. Does cognitive behavioral therapy change the brain? A systematic review of neuroimaging in anxiety disorders. J Neuropsychiatry Clin Neurosci 2009;21(2):114-125.

54. Clark DA, Beck AT. Cognitive theory and therapy of anxiety and depression: convergence with neurobiological findings. Trends Cogn Sci 2010;14(9):418-424.

55. Dissanayaka NNW, White E, O'Sullivan JD, et al. Characteristics and Treatment of Anxiety Disorders in Parkinson's Disease. Mov Disord Clin Pract 2015;2(2):155-162.

56. Bloem BR, Boers I, Cramer M, Westendorp RG, Gerschlager W. Falls in the elderly. I. Identification of risk factors. Wien Klin Wochenschr 2001;113(10):352-362.

57. Hirschtritt ME, Bloch MH, Mathews CA. Deep Brain Stimulation for Obsessive-Compulsive Disorder Reply. Jama-J Am Med Assoc 2017;318(4):393-393.

58. Burguiere E, Monteiro P, Mallet L, Feng GP, Graybiel AM. Striatal circuits, habits, and implications for obsessive-compulsive disorder. Current Opinion in Neurobiology 2015;30:59-65.

59. Thomsen PH. Obsessive-compulsive disorders. Eur Child Adoles Psy 2013;22:S23-S28.

60. Van Ameringen M, Patterson B, Simpson W. DSM-5 obsessive-compulsive and related disorders: clinical implications of new criteria. Depress Anxiety 2014;31(6):487-493.

61. Association AP. Diagnostic and Statistical Manual of Mental Disorders. 5th Edition. Washington, DC: American Psychiatric Association, 2013.

62. Whiteside SP, Port JD, Abramowitz JS. A meta-analysis of functional neuroimaging in obsessive-compulsive disorder. Psychiatry Res 2004;132(1):69-79.

63. Harrison BJ, Pujol J, Cardoner N, et al. Brain corticostriatal systems and the major clinical symptom dimensions of obsessive-compulsive disorder. Biol Psychiatry 2013;73(4):321-328.

64. Hou JM, Zhao M, Zhang W, et al. Resting-state functional connectivity abnormalities in patients with obsessive-compulsive disorder and their healthy first-degree relatives. J Psychiatry Neurosci 2014;39(5):304-311.

65. Sakai Y, Narumoto J, Nishida S, et al. Corticostriatal functional connectivity in non-medicated patients with obsessive-compulsive disorder. Eur Psychiatry 2011;26(7):463-469.

66. Beucke JC, Sepulcre J, Talukdar T, et al. Abnormally high degree connectivity of the orbitofrontal cortex in obsessive-compulsive disorder. JAMA Psychiatry 2013;70(6):619-629.

67. Lees AJ. The Neurobehavioural Abnormalities in Parkinson's Disease and their Relationship to Psychomotor Retardation and Obsessional Compulsive Disorders. Behav Neurol 1989;2(1):1-11.

68. Tomer R, Levin BE, Weiner WJ. Obsessive-Compulsive Symptoms and Motor Asymmetries in Parkinsons-Disease. Neuropsy Neuropsy Be 1993;6(1):26-30.

69. Alegret M, Junque C, Valldeoriola F, Vendrell P, Marti MJ, Tolosa E. Obsessive-compulsive symptoms in Parkinson's disease. J Neurol Neurosurg Psychiatry 2001;70(3):394-396.

70. Rosa-Alcazar Al, Sanchez-Meca J, Gomez-Conesa A, Marin-Martinez F. Psychological treatment of obsessive-compulsive disorder: a meta-analysis. Clin Psychol Rev 2008;28(8):1310-1325. 71. Ost LG, Havnen A, Hansen B, Kvale G. Cognitive behavioral treatments of obsessivecompulsive disorder. A systematic review and meta-analysis of studies published 1993-2014. Clin Psychol Rev 2015;40:156-169.

72. Hirschtritt ME, Bloch MH, Mathews CA. Obsessive-Compulsive Disorder: Advances in Diagnosis and Treatment. JAMA 2017;317(13):1358-1367. 
73. Cottraux J, Bouvard MA, Milliery M. Combining Pharmacotherapy with cognitive-behavioral interventions for obsessive-compulsive disorder. Cogn Behav Ther 2005;34(3):185-192.

74. Denys D. Pharmacotherapy of obsessive-compulsive disorder and obsessive-compulsive spectrum disorders. Psychiat Clin N Am 2006;29(2):553-+.

75. Parent A, Hazrati LN. Functional anatomy of the basal ganglia. II. The place of subthalamic nucleus and external pallidum in basal ganglia circuitry. Brain Res Brain Res Rev 1995;20(1):128-154.

76. Temel $Y$, Visser-Vandewalle V. Targets for deep brain stimulation in Parkinson's disease. Expert Opin Ther Tar 2006;10(3):355-362.

77. Temel Y. Limbic effects of high-frequency stimulation of the subthalamic nucleus. Vitam Horm 2010;82:47-63.

78. Abbes $\mathrm{M}$, Lhommee $\mathrm{E}$, Thobois $\mathrm{S}$, et al. Subthalamic stimulation and neuropsychiatric symptoms in Parkinson's disease: results from a long-term follow-up cohort study. J Neurol Neurosur Ps 2018;89(8):836-843.

79. Witt K, Daniels C, Volkmann J. Factors associated with neuropsychiatric side-effects after STNDBS in Parkinson's disease. Parkinsonism Relat Disord 2012;18 Suppl 1:S168-170.

80. Smeding HM, Speelman JD, Huizenga HM, Schuurman PR, Schmand B. Predictors of cognitive and psychosocial outcome after STN-DBS in Parkinson's Disease. J Neurol Neurosurg Psychiatry 2011;82(7):754-760.

81. Witt K, Granert O, Daniels C, et al. Relation of lead trajectory and electrode position to neuropsychological outcomes of subthalamic neurostimulation in Parkinson's disease: results from a randomized trial. Brain 2013;136(Pt 7):2109-2119.

82. Houeto JL, Mesnage V, Mallet L, et al. Behavioural disorders, Parkinson's disease and subthalamic stimulation. J Neurol Neurosurg Psychiatry 2002;72(6):701-707.

83. Faggiani E, Benazzouz A. Deep brain stimulation of the subthalamic nucleus in Parkinson's disease: From history to the interaction with the monoaminergic systems. Prog Neurobiol 2017;151:139156.

84. Temel Y, Boothman LJ, Blokland A, et al. Inhibition of 5-HT neuron activity and induction of depressive-like behavior by high-frequency stimulation of the subthalamic nucleus. Proc Natl Acad Sci U S A 2007;104(43):17087-17092.

85. Tan SK, Hartung H, Visser-Vandewalle V, Steinbusch HW, Temel Y, Sharp T. A combined in vivo neurochemical and electrophysiological analysis of the effect of high-frequency stimulation of the subthalamic nucleus on 5-HT transmission. Exp Neurol 2012;233(1):145-153.

86. Navailles S, Benazzouz A, Bioulac B, Gross C, De Deurwaerdere P. High-frequency stimulation of the subthalamic nucleus and L-3,4-dihydroxyphenylalanine inhibit in vivo serotonin release in the prefrontal cortex and hippocampus in a rat model of Parkinson's disease. J Neurosci 2010;30(6):23562364.

87. Mallet L, Mesnage V, Houeto JL, et al. Compulsions, Parkinson's disease, and stimulation. Lancet 2002;360(9342):1302-1304.

88. Fontaine $\mathrm{D}$, Mattei $\mathrm{V}$, Borg $\mathrm{M}$, et al. Effect of subthalamic nucleus stimulation on obsessivecompulsive disorder in a patient with Parkinson disease. Case report. J Neurosurg 2004;100(6):10841086.

89. Alonso P, Cuadras D, Gabriels L, et al. Deep Brain Stimulation for Obsessive-Compulsive Disorder: A Meta-Analysis of Treatment Outcome and Predictors of Response. Plos One 2015;10(7):e0133591.

90. Kisely S, Hall K, Siskind D, Frater J, Olson S, Crompton D. Deep brain stimulation for obsessivecompulsive disorder: a systematic review and meta-analysis. Psychol Med 2014;44(16):3533-3542. 



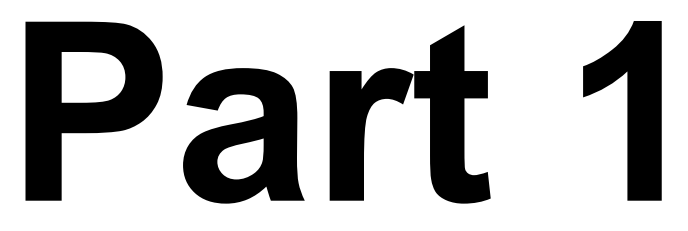

DBS studies in OCD and PD 



\section{2}

Deep brain stimulation of the subthalamic nucleus in obsessive-compulsive disorder: neuroanatomical and pathophysiological considerations

Anne Mulders, Birgit Plantinga, Koen Schruers, Annelien Duits, Marcus Janssen, Linda Ackermans, Albert Leentjens, Ali Jahanshahi, Yasin Temel European Neuropsychopharmacology (2016) 26, 1909 - 1919. 


\section{ABSTRACT}

Obsessive-compulsive disorder (OCD) is among the most disabling chronic psychiatric disorders and has a significant negative impact on multiple domains of quality of life. For patients suffering from severe refractory OCD, deep brain stimulation (DBS) of the subthalamic nucleus (STN) has been applied. Reviewing the literature of the last years we believe that through its central position within the cortico-basal gangliathalamocortical circuits, the STN has a coordinating role in decision-making and actionselection mechanisms. Dysfunctional information-processing at the level of the STN is responsible for some of the core symptoms of OCD. Research confirms an electrophysiological dysfunction in the associative and limbic (non-motor) parts of the STN. Compared to Parkinson's disease patients, STN neurons in OCD exhibit a lower firing rate, less frequent but longer bursts, increased burst activity in the anterior ventromedial area, an asymmetrical left-sided burst distribution, and a predominant oscillatory activity in the $\delta$-band. Moreover, there is direct evidence for the involvement of the STN in both checking behaviour and OCD symptoms, which are both related to changes in electrophysiological activity in the non-motor STN. Through a combination of mechanisms, DBS of the STN seems to interrupt the disturbed informationprocessing, leading to a normalisation of connectivity within the cortico-basal gangliathalamocortical circuits and consequently to a reduction in symptoms. In conclusion, based on the STN's strategic position within cortico-basal ganglia-thalamocortical circuits and its involvement in action-selection mechanisms that are responsible for some of the core symptoms of OCD, the STN is a mechanism-based target for DBS in OCD. 


\section{INTRODUCTION}

In the last decades the interest in neuromodulation therapies for psychiatric disorders, such as obsessive-compulsive disorder (OCD), has increased due to the advances in the field of deep brain stimulation (DBS). OCD is among the most disabling chronic psychiatric disorders and has a significant negative impact on multiple domains of quality of life ${ }^{1-3}$. Even after state-of-the-art psychotherapeutic and pharmacological treatments, approximately $10-20 \%$ of patients do not respond sufficiently to treatment ${ }^{4,5}$. For the most treatment-resistant OCD patients, DBS has resulted in a substantial relief of key symptoms ${ }^{6-8}$. The rationale for the current use of DBS for OCD is based on findings from both clinical and experimental studies, suggesting an involvement of abnormal functioning of cortico-basal ganglia-thalamocortical circuits $^{1,5,9}$. The main target areas for DBS for OCD are the ventral capsule/ventral striatum (VC/VS), the nucleus accumbens (NAc) with the involvement of the bed nucleus of the stria terminalis, the anterior limb of the internal capsule (ALIC), and the subthalamic nucleus (STN) 1, 8, 10,11. In this review we will focus on the latter target. The STN as a surgical target for OCD has recently been introduced and the therapeutic effects are promising ${ }^{3}, 12$. The STN is a key structure in the basal ganglia in which motor, cognitive and limbic projections come together ${ }^{13-15}$. It is like a crossroad in which different modalities of behaviour are being processed ${ }^{16}$. The present review aims the shed light on the functional role of the STN in the pathophysiology of OCD, by integrating neuroanatomical and pathophysiological findings.

\section{Obsessive-compulsive disorder}

OCD is characterised by the repeated occurrence of obsessions and/or compulsions that are recognised as excessive, inappropriate or unreasonable, causing marked distress, and significantly interfere with daily functioning ${ }^{1}$. Obsessions are defined as intrusive, repetitive and distressing thoughts, images, or impulses that can cause anxiety or distress. Compulsions can take the form of repetitive, stereotyped, or ritualised overt behaviours or covert mental acts, both of which perform a neutralisation function in order to reduce distress, to weaken an obsession, or to prevent some feared outcome $^{3,17}$. According to epidemiological studies, the prevalence of OCD in the general population and across countries has been estimated at approximately $1-3 \%{ }^{18}$. 
The treatment of OCD is based on assessment of the severity of OCD, age, and the presence of comorbid disorders ${ }^{17,18}$. Conventional treatments include psychoeducation, reduction of psychosocial stress, cognitive behavioural therapy (CBT) and/or pharmacotherapy $3,17,18$. Selective serotonin reuptake inhibitors (SSRIs) are the first choice of medication in $O C D^{17,19}$. However, the response to treatment can take months and high dosages are often required for good efficacy ${ }^{20,}{ }^{21}$. Moreover, up to $60 \%$ of OCD patients do not respond to treatment with SSRIs ${ }^{19,20,22}$.

\section{Deep brain stimulation}

In DBS, electrical current is delivered to specific locations in the brain through permanently implanted electrodes ${ }^{9}$. Because of its adjustable stimulation parameters, DBS therapy can be tailored for individual patients ${ }^{7}$. DBS is a treatment option only for OCD patients who have failed to respond to: (1) three treatment attempts with SSRIs, including clomipramine for at least 10-12 weeks at the maximum doses; (2) augmentation with a neuroleptic; and, (3) a minimum of $16-20$ sessions of $\mathrm{CBT}^{1,3}$.

To date more than 100 patients with refractory-OCD have received DBS in one of the following targets: the VC/NS; the inferior thalamic peduncle; the NAC; the ALIC; the medial forebrain bundle ${ }^{23}$; and, the STN (see ${ }^{1,8,10,24}$ ). The STN is a well-known target for DBS in patients with early and advanced Parkinson's disease (PD) ${ }^{25,26}$. The interest for the STN as a potential target for DBS in OCD came from case observations. In two patients with advanced PD and comorbid OCD, STN-DBS resulted in an improvement of the Parkinsonian symptoms and a substantial reduction of obsessive and compulsive symptoms ${ }^{27}$. A similar observation was published two years later ${ }^{28}$. Based on these findings and the long-term effects of stimulation of the STN in PD patients, a larger double-blind cross-over study of STN-DBS was performed in patients with refractory OCD in order to assess the efficacy and safety ${ }^{3}$. After three months of stimulation, $70 \%$ of patients with active stimulation showed a reduction of $35 \%$ or more of the Yale-Brown Obsessive-Compulsive Scale (Y-BOCS) and $62 \%$ of patients achieved satisfactory global functioning (as measured with the Global Assessment of Functioning scale (GAF)), compared to $12 \%$ of patients with sham stimulation. This led to the conclusion that STN-DBS can be an effective therapy for patients with refractory OCD. Complications included one intracerebral haemorrhage, two infections and three cases of transient, stimulation-induced hypomania ${ }^{3,29}$. Infections are not uncommon in 
OCD patients who have received DBS ${ }^{1}$. According to our clinical experience this is related to an increased tendency of OCD patients, compared to other patient groups, to touch their wounds. Table 1 summarises the main findings of studies with STN-DBS in OCD.

\section{Intrinsic organisation and connectivity of the STN}

The STN is a small, densely populated and highly vascularised lenticular nucleus ${ }^{15,30}$. It is located in the diencephalo-mesencephalic area rostral to the substantia nigra ${ }^{31}$, between the zona incerta medially/superiorly and the substantia nigra inferiorly. It is medially and laterally encapsulated by major fiber bundles, such as the medial forebrain bundle and the internal capsule, respectively. STN neurons are tightly placed next to each other, without any intervening glial membranes. Dendrites extend to a relatively small part of the STN ${ }^{32}$. It is believed that most STN neurons are long-axoned projection neurons that emerge directly from the cell soma ${ }^{15}$. It is the only glutamatergic nucleus in the basal ganglia.

The STN participates in cortico-basal ganglia-thalamocortical circuits that mediate motor, cognitive and emotional functioning ${ }^{14,15}$. The current idea is that these circuits are partially segregated and integrated ${ }^{33}$. In line with this, the STN can be divided into three functional divisions: a dorsolateral motor region, a ventromedial associative region, and the medial tip/rostral (limbic) region (see Fig. 1) 15, 32, 34, 35.

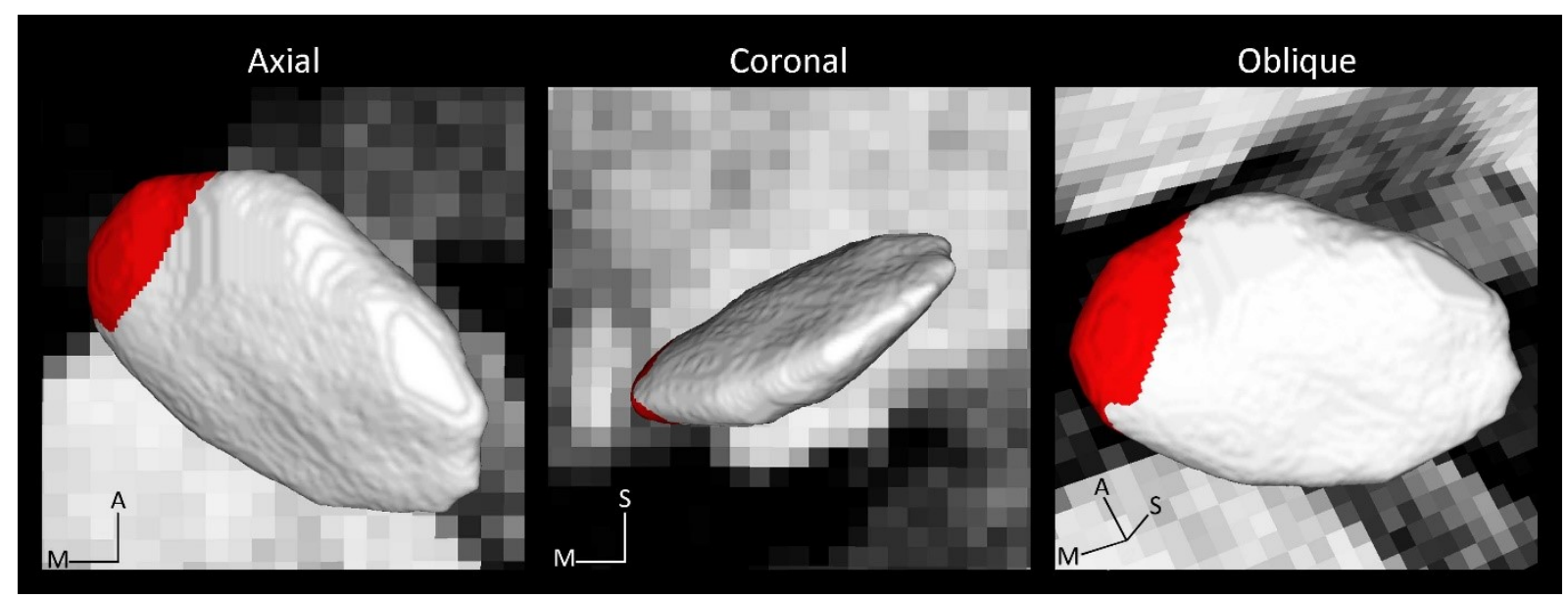

Figure 1. Three views of the limbic tip (red) of the right STN in one healthy subject, based on the STN's connectivity to limbic cortical areas as determined with diffusion weighted ultrahigh field MRI (7T, courtesy of $\mathrm{Y}$. Temel). $\mathrm{A}=$ anterior, $\mathrm{M}=$ medial, $\mathrm{S}=$ superior. 
Table 1. Reports of subthalamic nucleus-deep brain stimulation for obsessive-compulsive disorder

\begin{tabular}{|c|c|c|c|}
\hline Author & Patients & Effects & Side-effects/comment \\
\hline Mallet et al., 2002 & 2 & $\begin{array}{l}\text { Positive case } \\
\text { report }\end{array}$ & $\begin{array}{l}\text { Treatment not intended for OCD. } \\
\text { Beneficial effect of STN-DBS in patients } \\
\text { with Parkinson disease with comorbid } \\
\text { OCD }\end{array}$ \\
\hline Fontaine et al., 2004 & 1 & $\begin{array}{l}\text { Positive } \\
\text { report }\end{array}$ & $\begin{array}{l}\text { Beneficial effect of STN-DBS in a patient } \\
\text { with Parkinson disease with comorbid } \\
\text { OCD }\end{array}$ \\
\hline Mallet et al., 2008 & 17 & $\begin{array}{l}\text { Y-BOCS } \\
\text { reduced by } 41 \%\end{array}$ & $\begin{array}{l}1 \text { intracerebral haemorrhage with } \\
\text { permanent finger palsy, } 2 \text { infections, and } \\
\text { other reversible side-effects, including } \\
\text { hypomania }\end{array}$ \\
\hline Le Jeune et al., 2010 & $\begin{array}{l}10 \text { patients } \\
\text { included in } \\
\text { Mallet et al., } \\
2008\end{array}$ & & $\begin{array}{l}\text { Analysis of prefrontal metabolism after } \\
\text { STN-DBS in OCD in sham- and active } \\
\text { stimulation }\end{array}$ \\
\hline Piallat et al., 2011 & $\begin{array}{l}9 \text {, of which } 3 \\
\text { were included } \\
\text { in Mallet et al., } \\
2008\end{array}$ & & $\begin{array}{l}\text { No clinical data. Analysis of neuronal } \\
\text { firing }\end{array}$ \\
\hline Welter et al., 2011 & $\begin{array}{l}12 \text { patients } \\
\text { included in } \\
\text { Mallet et al., } \\
2008\end{array}$ & & $\begin{array}{l}\text { Analysis of subthalamic neuronal activity } \\
\text { in relation to obsessions and } \\
\text { compulsions severity }\end{array}$ \\
\hline $\begin{array}{l}\text { Chabardès et al., } \\
2012\end{array}$ & $\begin{array}{l}4 \text {, of which } 2 \\
\text { were included } \\
\text { in Mallet et al., } \\
2008\end{array}$ & $\begin{array}{l}\text { Benefit that can } \\
\text { reach } 50-75 \% \\
\text { on the Y-BOCS }\end{array}$ & $\begin{array}{l}\text { Weight-gain, voltage-dependent side- } \\
\text { effects, including hypomania and anxiety }\end{array}$ \\
\hline Barcia et al., 2014 & 2 & $\begin{array}{l}\text { Pre- and } \\
\text { postoperative } \\
\text { Y-BOCS scores } \\
\text { were } 33 \text { and } 16 \\
\text { respectively, } \\
\text { and } 33 \text { and } 3 \text {, } \\
\text { respectively }\end{array}$ & $\begin{array}{l}\text { Screening for the best target between } \\
\text { the right and left STN and NAc. In both } \\
\text { patients, the combination of electrodes, } \\
\text { which best relieved the OCD symptoms } \\
\text { was both the left STN and left NAc }\end{array}$ \\
\hline Bastin et al., 2014 & $\begin{array}{l}7, \text { of which } \\
\text { some were } \\
\text { included in } \\
\text { Mallet et al., } \\
2008 \text { and } \\
\text { Chabardès et } \\
\text { al., } 2013\end{array}$ & & $\begin{array}{l}\text { Analyses of electrophysiological } \\
\text { recordings of the STN during acute } \\
\text { symptoms of OCD }\end{array}$ \\
\hline Burbaud et al., 2014 & $\begin{array}{l}10 \text { patients } \\
\text { included in } \\
\text { Mallet et al., } \\
2008\end{array}$ & & $\begin{array}{l}\text { Analyses of subthalamic neuronal } \\
\text { activity while patients performed a } \\
\text { cognitive task }\end{array}$ \\
\hline \multicolumn{4}{|c|}{$\begin{array}{l}\text { This table summarised the studies reporting on the effects of DBS of the STN in patients with OCD. } \\
\text { The number of patients, the main effects and the side-effects are described. Some patients have been } \\
\text { included in more than } 1 \text { publication. In these cases, data are only presented once. DBS = deep brain } \\
\text { stimulation, STN = subthalamic nucleus, OCD = obsessive-compulsive disorder, NAc = nucleus } \\
\text { accumbens. }\end{array}$} \\
\hline
\end{tabular}


A dysfunction in the cortico-basal ganglia-thalamocortical limbic circuit, which processes motivational and affective processes, has especially been implicated in the pathophysiology of $\mathrm{OCD}^{24}, 36,37$. The limbic circuit consists of the hippocampal formation, the amygdala, the medial orbito-frontal cortex (OFC), the ventromedial prefrontal cortex (VmPFC), the anterior cingulate cortex (ACC), the VS, ventral pallidum and the dorsomedial nucleus of the thalamus (DM) ${ }^{13}, 15$. The medial tip of the STN is reciprocally connected with the ventral pallidum, also known as a major limbic output region ${ }^{15,38}$. The ventral pallidum, in turn, receives projections from the VS, which consists of the ventromedial portions of the caudate, the NAc, and the medium-celled portion of the olfactory tubercle. This indirectly connects the STN with the NAc. The VS receives excitatory projections from the hippocampal formation, the amygdala and the limbic and paralimbic cortices ${ }^{13}, 15,38$. The STN also receives direct cortical input, also known as the hyperdirect pathway ${ }^{39}$. Via the ventral pallidum, the limbic circuit is closed by a thalamo-cortical glutamateric pathway ${ }^{15}$.

\section{The behavioural role of the STN}

The STN has a pivotal place in a complex neuronal network in which indirect and hyperdirect pathways come together. The success and widespread use of the STN as a target for PD has provided the opportunity to record its electrical activity. This has contributed to a better understanding of the functional roles of the STN in the context of $P D^{40}$.

According to current computational models the STN acts by converging a variety of information from several cortical regions, and by establishing a decisional threshold in order to assess the amount of information needed for behavioural expression during situations involving conflict ${ }^{41}$. Multiple lines of evidence have consistently shown impaired response inhibition during conflict situations as assessed by different paradigms that require the withholding or inhibition of a prepotent motor response ${ }^{42-46}$. Moreover, electrophysiological and imaging studies suggest that the STN is involved in both reactive and proactive inhibition ${ }^{47}$, which are associated with changes in beta band activity $(13-35 \mathrm{~Hz})^{48,49}$. In addition, correlates of conflict in theta $(4-8 \mathrm{~Hz})$ and delta $(2-4 \mathrm{~Hz})$ bands of neuronal activity within the STN provided further evidence for the role of the STN in inhibitory processes ${ }^{50,51}$. 
Non-motor side-effects observed after STN-DBS in PD patients, such as impulse control disorders, (hypo)mania, and acute depression ${ }^{15,52,53}$, but also improvement in neuropsychiatric symptoms following STN-DBS ${ }^{54}$, indicated that the STN is not purely a motor structure, but is also involved in cognitive and emotional functioning. Indeed, deficits in non-motor inhibition have been demonstrated following DBS of the STN in $P D$, including increased errors on the Stroop interference task, which requires the suppression of habitual responses ${ }^{55}$. Another study showed changes in beta-band activity prior to response during incongruent trials in the Stroop task ${ }^{56}$.

Together with structures such as the right inferior frontal gyrus (rIFG) and presupplementary motor area (preSMA) ${ }^{49}, 57$, the STN seems to be essential in decision-making and action-selection processes, by its ability to stop or inhibit automatic and unwanted response outputs ${ }^{58,59}$. Interestingly, a recent study found synchronised activity in populations of STN neurons which reflected the subjective value of reward and the subjective cost of effort that could predict decision-making in an effort-based decision-making test ${ }^{60}$. Thus, instead of signaling conflict, STN LFP responses varied in proportion to subjective value, which led the authors to suggest that this STN signal may be understood in the context of task-engagement and resource allocation, thereby implying a role for the STN in motivational and rewardrelated processes ${ }^{60}$.

Findings from other studies that have used DBS to explore the role of the STN in emotional processing suggest that the STN is not just involved in specific categories or sub-processes of (different) emotions, but rather plays a more general coordinating role ${ }^{46}$. Similar to the computational models proposed for action and cognition as described previously, it is believed that the STN is involved in producing temporally organised neuronal co-activation patterns at the cortical and subcortical levels that are crucial for the regulation of thoughts and emotions ${ }^{46}$. Taken together, it appears that, through its central position in which motor, associative, and limbic circuits come together, the STN gathers different types of information on the environment to determine the required amount of information for any behavioural program to be expressed $^{29}$. 


\section{Electrophysiological recordings in the STN in patients with OCD}

Similar to studies in PD patients, single-neuron and local field potential (LFP) recordings in patients with $O C D$ are beginning to provide insight into the pathophysiology of OCD. Based on studies that found an electrophysiological signature in the dorsolateral (motor) region of the STN in patients with PD, including increased neuronal activity, and occurrence of bursts and oscillations ${ }^{61-63}$, some argued that in OCD an electrophysiological signature should be found in the ventromedial parts of the STN ${ }^{64,65}$. In one study, recordings of 9 OCD patients and 11 PD patients were analysed ${ }^{65}$ and in another study 12 patients with OCD and 12 patients with $\mathrm{PD}^{64}$. Both studies reported a lower mean STN firing rate in patients with OCD when compared to STN neurons of PD patients $(20.5 \pm 11.0 \mathrm{~Hz}$ vs $30.8 \pm 15.6 \mathrm{~Hz}$, respectively; and $22.4 \pm 13.7 \mathrm{~Hz}$ vs $31.6 \pm 13.5 \mathrm{~Hz}$, respectively), and a significantly higher intraburst frequency in PD than in OCD $(83.4 \pm 131.2 \mathrm{~Hz}$ vs $50.9 \pm 27.8 \mathrm{~Hz}$, respectively; and $74.4 \pm 34.7$ vs $53.7 \pm 52.6$, respectively) ${ }^{64,65}$. The mean interspike interval was also found to be significantly higher in OCD $(71.8 \pm 63.5 \mathrm{~ms})$, compared to PD $(40.1 \pm 26.3 \mathrm{~ms})^{64}$.

Interestingly, although the proportion of burst neurons did not differ significantly between OCD and PD patients, in $69 \%-67 \%^{64}$ and in $75 \%-73 \%{ }^{65}$, respectively, a significant difference in the proportion of bursty firing neurons within the non-motor STN and motor STN in OCD was found, being $83 \%$ and $50 \%$, respectively ${ }^{65}$. In contrast, in PD patients an opposite pattern was observed, with a burst proportion of $55 \%$ in the non-motor STN and a burst proportion of $81 \%$ in the motor STN ${ }^{65}$. In addition, in OCD patients the mean interspike interval of the STN neurons located in the motor part was found to be significantly higher compared to the mean interspike interval of the STN neurons located in the associative and limbic parts in OCD patients, and compared to all STN regions in PD patients ${ }^{64}$.

The observed mean burst duration of STN neurons in both studies was significantly higher in OCD patients compared to PD patients $(307.0 \pm 177.0$ s vs $243.0 \pm 131.0 \mathrm{~ms}$, respectively; and $823.5 \pm 665.0 \mathrm{~ms}$ vs $340.3 \pm 246.1 \mathrm{~ms}$, respectively) ${ }^{64,65}$. Furthermore, in OCD the proportion of burst neurons was asymmetrically distributed (83\% left side and $59 \%$ right side), whereas no asymmetry was found in $\mathrm{PD}^{65}$. 
Additionally, oscillatory activity in the STN was analysed, as well as the relationship between STN burst characteristics and OCD symptom severity and the clinical response to DBS ${ }^{64}$. Briefly, compared to the STN of PD patients, the proportion of neurons displaying oscillatory activity was lower in the STN of OCD patients $(46 \%$ in OCD vs $68 \%$ in PD), as well as the mean frequency in the $\beta$-low band $(12-20 \mathrm{~Hz})$ in STN neurons in OCD $(14.4 \pm 2.8 \mathrm{~Hz} \text { in OCD vs } 15.8 \pm 2.5 \mathrm{~Hz})^{64}$. Except for the presence of more $\theta$-band $(4-8 \mathrm{~Hz})$ activity in the STN of OCD patients and more $\beta$-low band activity in the STN of PD patients, the distribution of oscillatory activity was similar between OCD and PD patients ${ }^{64}$. Over the entire frequency range, more oscillatory activity was observed in the non-motor part of the STN in OCD, whereas in the STN of $\mathrm{PD}$ oscillatory activity was greater in the motor part ${ }^{64}$. Further, a relationship was found between STN neuronal activity and symptom severity and between STN neuronal activity and clinical improvements with DBS in OCD patients ${ }^{64}$. Specifically, the lower the burst duration and interburst interval, and the higher the mean discharge and intraburst frequencies, peak and power in the $\delta$-band $(1-4 \mathrm{~Hz})$, the more severe obsessions were. Moreover, the greater the intraburst frequency and the proportion of oscillatory activity in the $\alpha$-band $(8-12 \mathrm{~Hz})$, the more severe the compulsions were. Finally, patients with higher intraburst frequency, but with lower interburst interval had the best clinical outcome ${ }^{64}$.

Altogether, five main characteristics were identified that distinguished STN neurons in OCD from those in PD: (1) a lower firing rate, (2) a bursty pattern with less frequent but longer bursts, and lower intraburst frequency, (3) increased burst activity in the anterior ventromedial area, (4) an asymmetrical left-sided burst distribution, and (5) a predominant oscillatory activity in the $\delta$-band ${ }^{64}, 65$. Moreover, some burst characteristics were found to be related to OCD severity, and clinical improvement as a result of DBS seems to be dependent on STN neuronal activity, with a higher response for patients with higher intraburst frequency, but lower interburst interval ${ }^{64}$.

Although these findings strongly point into the direction of involvement of the non-motor part of the STN in OCD, one should be cautious in interpreting these characteristics as an electrophysiological signature of OCD. Firstly, in the absence of control data, it can be questioned whether the observed characteristics are unique to and specifically reflect OCD pathology. Secondly, while burst activity and oscillatory activity in the 
motor region of the STN in PD are related to a disturbed dopamine system ${ }^{61,66}$, we do not know how these two are related in OCD. Lastly, it should be taken into account that neurons within different sub-regions of the STN were recorded in OCD and PD patients respectively. Although the authors argued that it is unlikely that the observed differences between STN neurons in OCD and PD are due to morphological and physiological differences between these sub-regions ${ }^{64}$, the existence of neural clusters within the STN with different types of electrical properties has been proposed ${ }^{15,52}$.

Nonetheless, additional evidence for the involvement of the STN in OCD pathology came from studies that performed electrophysiological recordings of the STN in OCD during cognitive tasks and during obsessions. One study recorded STN activity in two patients during acute OCD symptoms that occurred during a stop-signal task, a few days after the implantation of the DBS leads in the non-motor part of the STN ${ }^{67}$. In comparison to five patients who did not show any acute OCD symptoms, significant increases of STN oscillations on the majority of STN contacts within the $\delta-\alpha(1-12 \mathrm{~Hz})$ frequency range were observed ${ }^{67}$. The authors suggested that this modified activity reflects a mixture of both pathological activity corresponding to repetitive unsuccessful action selection and compensatory mechanisms that reflect the maintenance of an over stable motor/cognitive/emotional set $^{67}$. Another single-neuron recording study of STN activity during a decision-making task in which OCD subjects had the possibility of unrestricted repetitive checking, revealed that checking behaviour favoured by doubt was associated with a higher STN firing rate in the non-motor part ${ }^{68}$, which confirms its role in repetitive doubt-related thinking, one of the main clinical characteristics of OCD $^{69}$.

\section{STN and OCD}

The involvement of the basal ganglia in the pathophysiology of OCD has been confirmed by multiple lines of research, including neuroimaging, neuropsychological, and electrophysiological studies ${ }^{90}$. However, there is still a lot of uncertainty with regard to the relationship between certain brain abnormalities and clinical OCD symptoms. Moreover, whereas the conventional view on OCD considers compulsions as a coping mechanism in order to neutralise distress and weaken obsessions ${ }^{3,17}$, it has been proposed that obsessions and obsession-related anxiety precede or follow, rather than cause compulsive behaviour ${ }^{70,71}$. Recent findings have shown that increased checking 
behaviour in OCD was unrelated to stimulus-evoked anxiety ${ }^{72}$. Accordingly, it has been suggested that compulsions represent the core feature of OCD, and result from a disruption of balance between goal-directed action and automatic habits ${ }^{70,73}$. As was pointed out before, the STN has a coordinating role in decision-making and actionselection mechanisms, by integrating multiple streams of information from the environment to determine the required amount of information necessary for behavioural expression ${ }^{41}$. At this point the question arises how neuronal activity within the non-motor part of the STN is related to OCD phenomenology.

Based on the electrophysiological data, there seems to be a disruption of the information-processing system of the associative and limbic circuits, at the level of the STN, in $O C D^{65,68}$. As already proposed by others, a dysfunction in this system could contribute to OCD symptomatology, such as increased checking behaviour or the incapacity to make a decision because of permanent doubt, since there is no smooth progression from a cognitive framework that is responsible for establishing priorities for potential behaviours to behavioural selection ${ }^{37}$. These anxiety-independent processes subsequently lead to unsuccessful action selection and freezing of thoughts ${ }^{65,67}$.

The disruption of information-processing could be the result of an imbalance between direct and indirect pathways, according to some similar to the dysfunction seen in movement disorders, such as $\mathrm{PD}^{74}$. While the direct pathway is thought to facilitate movement and the indirect pathway is thought to suppress unwanted movement, OCD can be conceptualised as the over-activation of the direct pathway with limited inhibition by the indirect pathway, only now focusing on cognitive and limbic functions ${ }^{29,74}$. It has been hypothesised that as a result of this imbalance, which is reflected by the hyperactivity of the non-motor part of the STN in OCD, the STN is incapable of inhibiting unwanted behavioural programs ${ }^{29,68}$. This is consistent with findings of impaired performance of patients with $\mathrm{OCD}$ on response inhibition, switching and interference tasks (see ${ }^{75,76}$ ). Consequently, no proper feedback signals are being sent out to the cortex, resulting in a state of permanent doubt, as well as in the perpetuation of pathological repetitive behaviours. How and whether the hyperdirect pathway is involved in these processes needs further investigation. 
Alternatively, checking compulsions could be seen as an attempt to gather more information to reach the threshold, whereas obsessional content would be created by the patients in order to rationalise the compulsions ${ }^{29}$, or explain their bad habits ${ }^{70,73}$. It is also possible that the STN's decisional threshold would not be reset after the expression of certain behaviour due to NAc dysfunction, which is, like the STN ${ }^{68}$, associated with impaired goal-directed motivated behaviour in $\mathrm{OCD}^{77}$. This dysfunction would in turn lead to repetitive behaviour observed in OCD patients through excessive fronto-striatal connectivity. The pathological doubt that occurs in OCD could finally be caused by cortical dysfunctions that modify the decisional threshold ${ }^{29}$.

Based on the electrophysiological evidence and the proposed role of the STN in OCD, we believe that STN-DBS interrupts the disturbed information-processing that seems to be responsible for the pathological repetitive behaviours characteristic for OCD by interfering with the pathological activity of the STN. As a consequence, other compensatory connective pathways can take over and normalise the feedback signals towards the cortex, resulting in a decrease in symptoms. In other words, local stimulation of the STN seems to restore the imbalance between the direct and indirect pathway, resulting in normalisation of the pathological connectivity within the corticobasal ganglia-thalamocortical circuit.

This mechanism, known as the disruption hypothesis ${ }^{78}$, is consistent with findings of decreased OFC and medial PFC metabolism, as well as ACC activity in OCD patients following STN-DBS ${ }^{79}$, confirming the distal effects of DBS. Likewise, it is consistent with the symptom-related changes observed in the STN in OCD, which suggest that DBS might directly act on pathological STN activity ${ }^{64}$. Moreover, the idea that DBS acts on neural connectivity can account for findings of similar percentages of improvement of DBS in the different target areas in patients with OCD, which are all part of the proposed dysfunctional circuit ${ }^{10,80}$. It has been suggested that underlying mechanisms of DBS are largely independent of the target ${ }^{81}$, and that DBS resets the network dynamics within a circuit by functionally overriding disruptive neuronal activity between different brain network nodes ${ }^{82}$ that are assumed to be involved in the disorder. For OCD, examples include the involvement of the NAc in goal-directed motivated behaviour ${ }^{77}$, and the role of the caudate nucleus in error-detection ${ }^{83}$. 
In fact, there is currently no consensus with regard to the optimal anatomical target for $O C D^{10,80}$. It can be argued that other commonly used targets, including the ALIC, VC/VS and NAc, are more or less part of one and the same larger region. That is, DBS at the ALIC, which is part of the internal capsule, was found to be most successful when more ventral regions are targeted, which resulted in the exploration of the VC/VS as target for $\mathrm{OCD}^{84,85}$. For VC/VS stimulation, the most ventral contact is usually placed in the NAC area, whereas the middle contacts are placed to stimulate the ventral portion of the ALIC ${ }^{86}$. For stimulation of the NAc, which is located just beneath the ALIC at the head of the caudate and the anterior portion of the putamen, dorsal contacts at the ventral border of the internal capsule seem to be involved in more favourable improvements ${ }^{86-89}$. The use of larger electrodes (model 3387, Medtronic, Minneapolis, $\mathrm{MN}$ ) for these regions can benefit the search for the target with the greatest benefit for each patient. However, research has also shown that there is a high variability of contact placement ${ }^{89}$, which makes it difficult to compare the efficacy of the different structures in the search for the best target area for DBS in OCD. On the contrary, the STN is a relative small nucleus. Based on its central position within the cortico-basal ganglia-thalamocortical circuits, its coordinating role in emotional and cognitive functioning, its established involvement in processes related to $O C D$, and its familiarity as a target for PD, we propose that the STN can be considered a favourable and mechanism-based target for DBS in OCD (Fig. 2).

\section{CONCLUSION}

Although literature on the specific role of the STN in OCD circuitry and its pathophysiological mechanisms is limited, research findings are indicative of an electrophysiological dysfunction in the non-motor part of the STN, which correlates with symptom severity and the response to STN-DBS. Moreover, there is direct evidence for the involvement on the non-motor STN in both checking behaviour and OCD symptoms, which are both related to changes in electrophysiological activity in this region. These findings are in line with proposed models of decision-making, suggesting a key role of the STN in the integration of various types of information which is necessary for establishing priorities for potential behaviour and action-planning, as well as for the coordination of action, stream of thoughts and motivation ${ }^{29,37}$. We believe that STN dysfunction leads to a disruption of information-processing of associative and 
limbic processes, hence leading to obsessions and compulsions. Through combination of mechanisms DBS of the STN interrupts this disturbed informationprocessing by interfering with pathological activity of the STN and by facilitating compensatory connective pathways to normalise the feedback signals that are sent towards the cortex, eventually leading to symptom reduction.

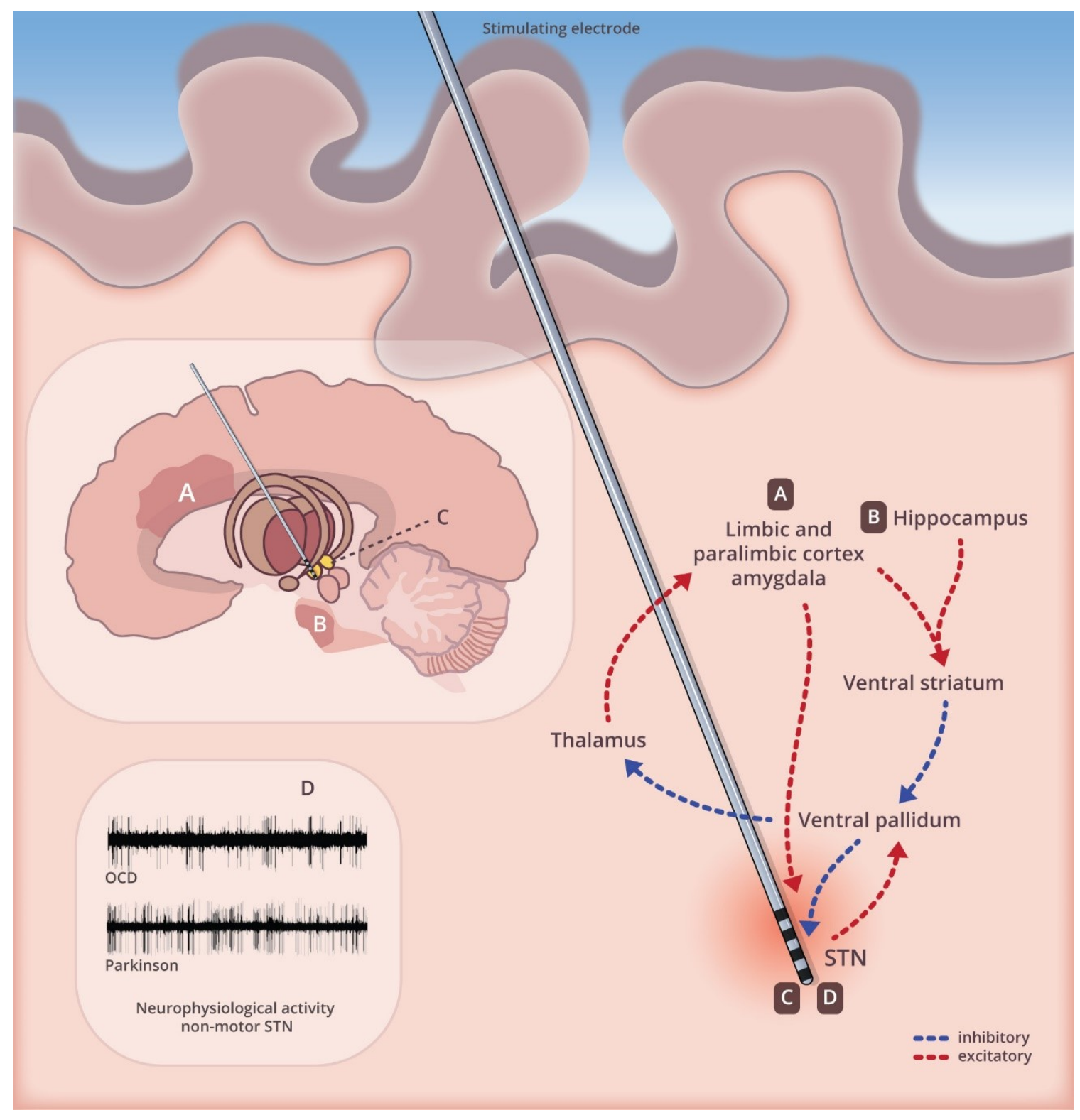

Figure 2. Schematic illustration of the subthalamic nucleus (STN) within the primate limbic circuit in obsessive-compulsive disorder (OCD).

Projections from the limbic and paralimbic cortices, the amygdala (A) and hippocampus (B) are concentrated at the level of the ventral striatum. From the ventral striatum, the circuit is directed to the ventral pallidum. The medial tip of the STN $(\mathbf{C})$ is reciprocally connected with the ventral pallidum, also known as the major limbic output area. Via the ventral pallidum, the limbic circuit is closed by a thalamocortical glutamateric pathway. The STN also receives direct cortical input. Compared to Parkinson's disease, neurons in the associative and limbic part of the STN (D) exhibit a lower firing rate, less frequent but longer bursts, and an increased burst activity in OCD. 


\section{REFERENCES}

1. Blomstedt P, Sjoberg RL, Hansson M, Bodlund O, Hariz MI. Deep brain stimulation in the treatment of obsessive-compulsive disorder. World neurosurgery 2013;80(6):e245-253.

2. Hou SY, Yen CF, Huang MF, Wang PW, Yeh YC. Quality of life and its correlates in patients with obsessive-compulsive disorder. The Kaohsiung journal of medical sciences 2010;26(8):397-407.

3. Mallet L, Polosan M, Jaafari N, et al. Subthalamic nucleus stimulation in severe obsessivecompulsive disorder. The New England journal of medicine 2008;359(20):2121-2134.

4. De Koning P, Van den Munckhof P, Figee M, Schuurman R. Deep brain stimulation in obsessive-compulsive disorder targeted at the nucleus accumbens. In: Denys D, Feenstra $M$, Schuurman R, eds. Deep brain stimulation: a new frontier in psychiatry. Berlin Heidelberg: Springer, 2012:35-41.

5. Temel $\mathrm{Y}$, Hescham SA, Jahanshahi $A$, et al. Neuromodulation in psychiatric disorders. International review of neurobiology 2012;107:283-314.

6. Nuttin B, Cosyns P, Demeulemeester H, Gybels J, Meyerson B. Electrical stimulation in anterior limbs of internal capsules in patients with obsessive-compulsive disorder. Lancet 1999;354(9189):1526.

7. Greenberg BD, Rauch SL, Haber SN. Invasive circuitry-based neurotherapeutics: stereotactic ablation and deep brain stimulation for OCD. Neuropsychopharmacology 2010;35(1):317-336.

8. Kisely S, Hall K, Siskind D, Frater J, Olson S, Crompton D. Deep brain stimulation for obsessivecompulsive disorder: a systematic review and meta-analysis. Psychological medicine 2014;44(16):3533-3542.

9. Hamani C, Temel Y. Deep brain stimulation for psychiatric disease: contributions and validity of animal models. Science translational medicine 2012;4(142):142rv148.

10. Alonso P, Cuadras D, Gabriels L, et al. Deep Brain Stimulation for Obsessive-Compulsive Disorder: A Meta-Analysis of Treatment Outcome and Predictors of Response. PloS one 2015;10(7):e0133591.

11. Barcia JA, Reyes $L$, Arza $R$, et al. Deep brain stimulation for obsessive-compulsive disorder: is the side relevant? Stereotactic and functional neurosurgery 2014;92(1):31-36.

12. Chabardes $S$, Polosan $M$, Krack $P$, et al. Deep brain stimulation for obsessive-compulsive disorder: subthalamic nucleus target. World neurosurgery 2013;80(3-4):S31 e31-38.

13. Alexander GE, Crutcher MD. Functional architecture of basal ganglia circuits: neural substrates of parallel processing. Trends in neurosciences 1990;13(7):266-271.

14. Alexander GE, DeLong MR, Strick PL. Parallel organization of functionally segregated circuits linking basal ganglia and cortex. Annual review of neuroscience 1986;9:357-381.

15. Temel Y, Blokland A, Steinbusch HW, Visser-Vandewalle V. The functional role of the subthalamic nucleus in cognitive and limbic circuits. Prog Neurobiol 2005;76(6):393-413.

16. Mallet L, Schupbach M, N'Diaye K, et al. Stimulation of subterritories of the subthalamic nucleus reveals its role in the integration of the emotional and motor aspects of behavior. Proc Natl Acad Sci U S A 2007;104(25):10661-10666.

17. Schruers K, Koning K, Luermans J, Haack MJ, Griez E. Obsessive-compulsive disorder: a critical review of therapeutic perspectives. Acta psychiatrica Scandinavica 2005;111(4):261-271.

18. Thomsen $\mathrm{PH}$. Obsessive-compulsive disorders. European child \& adolescent psychiatry 2013;22 Suppl 1:S23-28.

19. Sayyah M, Majzoob S, Sayyah M. Metabolic and toxicological considerations for obsessivecompulsive disorder drug therapy. Expert opinion on drug metabolism \& toxicology 2013;9(6):657-673.

20. Pallanti S, Quercioli L. Treatment-refractory obsessive-compulsive disorder: methodological issues, operational definitions and therapeutic lines. Progress in neuro-psychopharmacology \& biological psychiatry 2006;30(3):400-412.

21. Brakoulias $V$. The future of pharmacotherapy for obsessive-compulsive disorder may lie in a better understanding of its heterogeneity. Expert opinion on pharmacotherapy 2014;15(10):1321-1323.

22. Dold M, Aigner M, Lanzenberger R, Kasper S. Antipsychotic augmentation of serotonin reuptake inhibitors in treatment-resistant obsessive-compulsive disorder: a meta-analysis of double-blind, 
randomized, placebo-controlled trials. The international journal of neuropsychopharmacology / official scientific journal of the Collegium Internationale Neuropsychopharmacologicum 2013;16(3):557-574.

23. Coenen VA, Schlaepfer TE, Goll P, et al. The medial forebrain bundle as a target for deep brain stimulation for obsessive-compulsive disorder. CNS spectrums 2016:1-8.

24. Lapidus KA, Kopell BH, Ben-Haim S, Rezai AR, Goodman WK. History of psychosurgery: a psychiatrist's perspective. World neurosurgery 2013;80(3-4):S27 e21-16.

25. Odekerken VJ, van Laar T, Staal MJ, et al. Subthalamic nucleus versus globus pallidus bilateral deep brain stimulation for advanced Parkinson's disease (NSTAPS study): a randomised controlled trial. The Lancet Neurology 2013;12(1):37-44.

26. Deuschl G, Schade-Brittinger C, Krack P, et al. A randomized trial of deep-brain stimulation for Parkinson's disease. New Engl J Med 2006;355(9):896-908.

27. Mallet L, Mesnage V, Houeto JL, et al. Compulsions, Parkinson's disease, and stimulation. Lancet 2002;360(9342):1302-1304.

28. Fontaine $\mathrm{D}$, Mattei $\mathrm{V}$, Borg $\mathrm{M}$, et al. Effect of subthalamic nucleus stimulation on obsessivecompulsive disorder in a patient with Parkinson disease. Case report. Journal of neurosurgery 2004;100(6):1084-1086.

29. Haynes $W$, Mallet $L$. What is the role of the subthalamic nucleus in obsessive-compulsive disorder? Elements and insights from deep brain stimulation studies. In: Denys D, Feenstra M, schuurman R, eds. Deep brain stimulation A new frontier in psychiatry. Berlin Heidelberg: Springer, 2012:53-59.

30. Rong J, Wang Q, Liu K, et al. A new atlas localization approach for subthalamic nucleus utilizing Chinese visible human head datasets. PloS one 2013;8(2):e57264.

31. Lanciego JL, Luquin N, Obeso JA. Functional neuroanatomy of the basal ganglia. Cold Spring Harbor perspectives in medicine 2012;2(12):a009621.

32. Parent A, Hazrati LN. Functional anatomy of the basal ganglia. II. The place of subthalamic nucleus and external pallidum in basal ganglia circuitry. Brain research Brain research reviews 1995;20(1):128-154.

33. Haynes WI, Haber SN. The organization of prefrontal-subthalamic inputs in primates provides an anatomical substrate for both functional specificity and integration: implications for basal ganglia models and deep brain stimulation. The Journal of neuroscience : the official journal of the Society for Neuroscience 2013;33(11):4804-4814.

34. Hamani C, Saint-Cyr JA, Fraser J, Kaplitt M, Lozano AM. The subthalamic nucleus in the context of movement disorders. Brain : a journal of neurology 2004;127(Pt 1):4-20.

35. Lambert C, Zrinzo L, Nagy Z, et al. Confirmation of functional zones within the human subthalamic nucleus: patterns of connectivity and sub-parcellation using diffusion weighted imaging. Neurolmage 2012;60(1):83-94.

36. Bourne SK, Eckhardt CA, Sheth SA, Eskandar EN. Mechanisms of deep brain stimulation for obsessive compulsive disorder: effects upon cells and circuits. Frontiers in integrative neuroscience 2012;6:29.

37. Graybiel AM, Rauch SL. Toward a neurobiology of obsessive-compulsive disorder. Neuron 2000;28(2):343-347.

38. Parent A, Hazrati LN. Functional anatomy of the basal ganglia. I. The cortico-basal gangliathalamo-cortical loop. Brain research Brain research reviews 1995;20(1):91-127.

39. Nambu A, Tokuno H, Takada M. Functional significance of the cortico-subthalamo-pallidal 'hyperdirect' pathway. Neuroscience research 2002;43(2):111-117.

40. Weinberger M, Hutchison WD, Dostrovsky JO. Pathological subthalamic nucleus oscillations in PD: can they be the cause of bradykinesia and akinesia? Experimental neurology 2009;219(1):58-61.

41. Frank MJ. Hold your horses: a dynamic computational role for the subthalamic nucleus in decision making. Neural networks : the official journal of the International Neural Network Society 2006;19(8):1120-1136.

42. Frank MJ, Samanta J, Moustafa AA, Sherman SJ. Hold your horses: impulsivity, deep brain stimulation, and medication in parkinsonism. Science 2007;318(5854):1309-1312. 
43. Witt K, Pulkowski U, Herzog J, et al. Deep brain stimulation of the subthalamic nucleus improves cognitive flexibility but impairs response inhibition in Parkinson disease. Archives of neurology 2004;61(5):697-700.

44. Hershey T, Campbell MC, Videen TO, et al. Mapping Go-No-Go performance within the subthalamic nucleus region. Brain : a journal of neurology 2010;133(Pt 12):3625-3634.

45. Green N, Bogacz R, Huebl J, Beyer AK, Kuhn AA, Heekeren HR. Reduction of influence of task difficulty on perceptual decision making by STN deep brain stimulation. Current biology : CB 2013;23(17):1681-1684.

46. Peron J, Fruhholz S, Verin M, Grandjean D. Subthalamic nucleus: a key structure for emotional component synchronization in humans. Neuroscience and biobehavioral reviews 2013;37(3):358-373.

47. Ballanger B, van Eimeren $T$, Moro E, et al. Stimulation of the subthalamic nucleus and impulsivity: release your horses. Ann Neurol 2009;66(6):817-824.

48. Benis D, David O, Lachaux JP, et al. Subthalamic nucleus activity dissociates proactive and reactive inhibition in patients with Parkinson's disease. Neurolmage 2014;91:273-281.

49. Swann N, Poizner H, Houser M, et al. Deep brain stimulation of the subthalamic nucleus alters the cortical profile of response inhibition in the beta frequency band: a scalp EEG study in Parkinson's disease. The Journal of neuroscience : the official journal of the Society for Neuroscience 2011;31(15):5721-5729.

50. Cavanagh JF, Wiecki TV, Cohen MX, et al. Subthalamic nucleus stimulation reverses mediofrontal influence over decision threshold. Nature neuroscience 2011;14(11):1462-1467.

51. Zavala B, Brittain JS, Jenkinson N, et al. Subthalamic nucleus local field potential activity during the Eriksen flanker task reveals a novel role for theta phase during conflict monitoring. The Journal of neuroscience : the official journal of the Society for Neuroscience 2013;33(37):14758-14766.

52. Broen M, Duits A, Visser-Vandewalle V, Temel Y, Winogrodzka A. Impulse control and related disorders in Parkinson's disease patients treated with bilateral subthalamic nucleus stimulation: a review. Parkinsonism Relat Disord 2011;17(6):413-417.

53. Temel Y, Kessels A, Tan S, Topdag A, Boon P, Visser-Vandewalle V. Behavioural changes after bilateral subthalamic stimulation in advanced Parkinson disease: a systematic review. Parkinsonism Relat Disord 2006;12(5):265-272.

54. Lhommee E, Klinger $\mathrm{H}$, Thobois $\mathrm{S}$, et al. Subthalamic stimulation in Parkinson's disease: restoring the balance of motivated behaviours. Brain : a journal of neurology 2012;135(Pt 5):1463-1477. 55. Jahanshahi M, Ardouin CM, Brown RG, et al. The impact of deep brain stimulation on executive function in Parkinson's disease. Brain : a journal of neurology 2000;123 ( $\mathrm{Pt}$ 6):1142-1154.

56. Brittain JS, Watkins KE, Joundi RA, et al. A role for the subthalamic nucleus in response inhibition during conflict. The Journal of neuroscience : the official journal of the Society for Neuroscience 2012;32(39):13396-13401.

57. Jahfari S, Waldorp L, van den Wildenberg WP, Scholte HS, Ridderinkhof KR, Forstmann BU. Effective connectivity reveals important roles for both the hyperdirect (fronto-subthalamic) and the indirect (fronto-striatal-pallidal) fronto-basal ganglia pathways during response inhibition. The Journal of neuroscience : the official journal of the Society for Neuroscience 2011;31(18):6891-6899.

58. Zavala B, Zaghloul K, Brown P. The subthalamic nucleus, oscillations, and conflict. Movement disorders : official journal of the Movement Disorder Society 2015;30(3):328-338.

59. Krack P, Hariz MI, Baunez C, Guridi J, Obeso JA. Deep brain stimulation: from neurology to psychiatry? Trends in neurosciences 2010;33(10):474-484.

60. Zenon A, Duclos Y, Carron R, et al. The human subthalamic nucleus encodes the subjective value of reward and the cost of effort during decision-making. Brain : a journal of neurology 2016;139(Pt 6):1830-1843.

61. Bergman H, Wichmann T, Karmon B, DeLong MR. The primate subthalamic nucleus. II. Neuronal activity in the MPTP model of parkinsonism. Journal of neurophysiology 1994;72(2):507-520. 62. Hutchison WD, Allan RJ, Opitz $\mathrm{H}$, et al. Neurophysiological Identification of the Subthalamic Nucleus in Surgery for Parkinson's Disease. Ann Neurol 1998;44(4):622-628. 
63. Rodrquez-Oroz MC, Rodriquez M, Guridi J, et al. The subthalamic nucleus in Parkinson's disease: somatotopic organization and physiological characteristics. Brain : a journal of neurology 2001;124:1777-1790.

64. Welter ML, Burbaud P, Fernandez-Vidal S, et al. Basal ganglia dysfunction in OCD: subthalamic neuronal activity correlates with symptoms severity and predicts high-frequency stimulation efficacy. Translational psychiatry 2011;1:e5.

65. Piallat $B$, Polosan $M$, Fraix $V$, et al. Subthalamic neuronal firing in obsessive-compulsive disorder and Parkinson disease. Ann Neurol 2011;69(5):793-802.

66. Brown P. Bad oscillations in Parkinson's disease. Journal of neural transmission Supplementum 2006(70):27-30.

67. Bastin J, Polosan M, Piallat B, et al. Changes of oscillatory activity in the subthalamic nucleus during obsessive-compulsive disorder symptoms: Two case reports. Cortex; a journal devoted to the study of the nervous system and behavior 2014.

68. Burbaud P, Clair AH, Langbour N, et al. Neuronal activity correlated with checking behaviour in the subthalamic nucleus of patients with obsessive-compulsive disorder. Brain : a journal of neurology 2013;136(Pt 1):304-317.

69. Tolin DF, Abramowitz JS, Brigidi BD, Foa EB. Intolerance of uncertainty in obsessivecompulsive disorder. Journal of anxiety disorders 2003;17(2):233-242.

70. Gillan CM, Morein-Zamir S, Urcelay GP, et al. Enhanced avoidance habits in obsessivecompulsive disorder. Biological psychiatry 2014;75(8):631-638.

71. Gillan CM, Robbins TW. Goal-directed learning and obsessive-compulsive disorder. Philosophical transactions of the Royal Society of London Series B, Biological sciences 2014;369(1655).

72. Clair AH, N'Diaye $\mathrm{K}$, Baroukh $\mathrm{T}$, et al. Excessive checking for non-anxiogenic stimuli in obsessive-compulsive disorder. European psychiatry : the journal of the Association of European Psychiatrists 2013;28(8):507-513.

73. Gillan CM, Sahakian BJ. Which is the driver, the obsessions or the compulsions, in OCD? Neuropsychopharmacology 2015;40(1):247-248.

74. Lipsman N, Neimat JS, Lozano AM. Deep brain stimulation for treatment-refractory obsessivecompulsive disorder: the search for a valid target. Neurosurgery 2007;61(1):1-11; discussion 11-13.

75. van Velzen LS, Vriend C, de Wit SJ, van den Heuvel OA. Response inhibition and interference control in obsessive-compulsive spectrum disorders. Frontiers in human neuroscience 2014;8:419.

76. Chamberlain SR, Blackwell AD, Fineberg NA, Robbins TW, Sahakian BJ. The neuropsychology of obsessive compulsive disorder: the importance of failures in cognitive and behavioural inhibition as candidate endophenotypic markers. Neuroscience and biobehavioral reviews 2005;29(3):399-419.

77. Figee M, Luigjes J, Smolders R, et al. Deep brain stimulation restores frontostriatal network activity in obsessive-compulsive disorder. Nature neuroscience 2013;16(4):386-387.

78. Chiken S, Nambu A. Mechanism of Deep Brain Stimulation: Inhibition, Excitation, or Disruption? The Neuroscientist : a review journal bringing neurobiology, neurology and psychiatry 2015.

79. Le Jeune F, Verin M, N'Diaye K, et al. Decrease of prefrontal metabolism after subthalamic stimulation in obsessive-compulsive disorder: a positron emission tomography study. Biological psychiatry 2010;68(11):1016-1022.

80. Kohl S, Schonherr DM, Luigjes J, et al. Deep brain stimulation for treatment-refractory obsessive compulsive disorder: a systematic review. BMC psychiatry 2014;14:214.

81. Benabid AL, Chabardes S, Mitrofanis J, Pollak P. Deep brain stimulation of the subthalamic nucleus for the treatment of Parkinson's disease. The Lancet Neurology 2009;8(1):67-81.

82. McIntyre CC, Hahn PJ. Network perspectives on the mechanisms of deep brain stimulation. Neurobiology of disease 2010;38(3):329-337.

83. Guehl D, Benazzouz A, Aouizerate B, et al. Neuronal correlates of obsessions in the caudate nucleus. Biological psychiatry 2008;63(6):557-562.

84. Greenberg BD, Malone DA, Friehs GM, et al. Three-year outcomes in deep brain stimulation for highly resistant obsessive-compulsive disorder. Neuropsychopharmacology 2006;31(11):2384-2393. 
85. Goodman WK, Foote KD, Greenberg BD, et al. Deep brain stimulation for intractable obsessive compulsive disorder: pilot study using a blinded, staggered-onset design. Biological psychiatry 2010;67(6):535-542.

86. Morishita T, Fayad SM, Goodman WK, et al. Surgical neuroanatomy and programming in deep brain stimulation for obsessive compulsive disorder. Neuromodulation : journal of the International Neuromodulation Society 2014;17(4):312-319; discussion 319.

87. Sturm V, Lenartz D, Koulousakis A, et al. The nucleus accumbens: a target for deep brain stimulation in obsessive-compulsive- and anxiety-disorders. Journal of chemical neuroanatomy 2003;26(4):293-299.

88. van den Munckhof P, Bosch DA, Mantione MH, Figee M, Denys DA, Schuurman PR. Active stimulation site of nucleus accumbens deep brain stimulation in obsessive-compulsive disorder is localized in the ventral internal capsule. Acta neurochirurgica Supplement 2013;117:53-59.

89. van den Munckhof P, Contarino MF, Bour LJ, Speelman JD, de Bie RM, Schuurman PR. Postoperative curving and upward displacement of deep brain stimulation electrodes caused by brain shift. Neurosurgery 2010;67(1):49-53; discussion 53-44.

90. Nakao T, Okada K, Kanba S. Neurobiological model of obsessive-compulsive disorder: evidence from recent neuropsychological and neuroimaging findings. Psychiatry Clinical Neuroscience 2014;68:587-605. 



\section{3}

Choreatic side-effects of deep brain stimulation of the anteromedial subthalamic nucleus for treatment resistant obsessive-compulsive disorder

Anne Mulders, Albert Leentjens, Koen Schruers, Annelien Duits, Linda Ackermans, Yasin Temel

World Neurosurgery (2017)104:1048.e9-1048.e13. 


\section{ABSTRACT}

Background: patients with treatment-resistant obsessive-compulsive disorder (OCD) are potential candidates for deep brain stimulation (DBS). The anteromedial subthalamic nucleus (STN) is amongst the most commonly used targets for DBS in OCD.

Case description: we present a patient with a 30-year history of treatment-resistant OCD who underwent anteromedial STN-DBS. Despite a clear mood-enhancing effect, stimulation caused motor side-effects including bilateral hyperkinesia, dyskinesias and sudden large amplitude choreatic movements of arms and legs when stimulating at voltages above circa $1.5 \mathrm{~V}$. DBS at lower amplitudes and at other contact points failed to result in a significant reduction of obsessions and compulsions without inducing motor side-effects. Due to this limitation in programming options, we decided to reoperate and target the ventral capsule/ventral striatum (VC/VS), which resulted in a substantial reduction in key obsessive and compulsive symptoms without serious sideeffects.

Conclusion: choreatic movements and hemiballismus have previously been linked to STN dysfunction and have been incidentally reported as side-effect of DBS of the dorsolateral STN in Parkinson's disease (PD). However, in PD, these side-effects were usually transient and rarely interfered with DBS programming. In our case, the motor side-effects were persistent and made optimal DBS programming impossible. To our knowledge such severe and persistent motor side-effects have not yet been described for anteromedial STN-DBS. 


\section{BACKGROUND}

Obsessive-compulsive disorder (OCD) is characterised by the repeated occurrence of upsetting obsessions and/or compulsions which are related to substantial dysfunction in multiple domains of life ${ }^{1}$. Despite intensive psychotherapeutic and pharmacological treatments, a significant proportion of patients fail to respond to therapy ${ }^{2}$. Patients with treatment-resistant OCD are potential candidates for deep brain stimulation (DBS) ${ }^{3}$. The ventral capsule/ventral striatum (VC/VS), the nucleus accumbens (NAc) and the anteromedial limbic portion of the subthalamic nucleus (STN) are amongst the most common DBS targets for OCD ${ }^{1}$. The aim of DBS is to tailor the treatment on a patientspecific basis in order to deliver optimal therapeutic effects while avoiding stimulationassociated side-effects. At present, minor complications have been reported of which the majority were time-limited and reversed by adjusting the stimulation settings ${ }^{1,4,5}$. We present significant stimulation-associated side-effects following DBS of the anteromedial STN not previously described and requiring a reimplantation to an alternative target, the VC/VS.

\section{CASE PRESENTATION}

A 47-year old woman with refractory OCD was referred to our hospital for DBS. The patient had been suffering from OCD since the age of 15 , which was clinically characterised by intrusive thoughts about dirt, accompanied with excessive cleaning, washing and checking compulsions that occupied the entire day. Additionally, the patient suffered from a lowered mood, anhedonia, and occasional panic attacks, though failing to meet the criteria for major depressive disorder or any specific anxiety disorder. The patient also showed signs of borderline personality disorder. She had received several psychological and pharmacological treatments without any longlasting relief on symptoms (Table 1). The patient was informed of the risks and benefits of DBS and underwent extensive multidisciplinary evaluation prior to surgery to ensure her suitability and compatibility for the treatment. The presurgery score on the YaleBrown Obsessive Compulsive Scale (Y-BOCS) ${ }^{6}$ was 34.

In September 2012, at age 49, DBS electrodes (Model 3389 DBS lead; Medtronic, MN, USA) were implanted bilaterally in the anteromedial portion of the STN using a CT/MRI fusion based stereotactic approach combined with intraoperative electrophysiological recordings (MER) with four electrodes (anterior, medial, central and lateral). MER 
showed approximately $5 \mathrm{~mm}$ of typical STN activity along the central trajectory for both left and right side, which was characterised by increased background activity, highamplitude discharges, and bursty firing neurons. Postoperative imaging validated appropriate placement of the electrodes (Figure 1). For final target coordinates see Table 1. Stimulation parameters were as follows: monopolar stimulation (left 1-, right 9-, case + ), a pulse width of 90 milliseconds, a frequency of $130 \mathrm{~Hz}$ and an amplitude of $1.5 \mathrm{~V}$ which had been slowly increased.

After activating the DBS therapy, the patient exhibited an immediate mood-enhancing effect and relief in obsessions and compulsions. However, the patient developed unexpected compulsive behaviour towards the operation wounds (scratching and itching), which has likely contributed to the development of a hardware infection. The infection was treated with removing the infected hardware (implanted pulse generator and cables) and antibiotic therapy. Two months later new hardware was implanted and DBS therapy reactivated. After this period, the mood-enhancing effect decreased and the programming was limited by the fact that STN-DBS caused motor side-effects at stimulation above circa 1.5V. The motor side-effects consisted of bilateral hyperkinesia and dyskinesias, as well as sudden large amplitude choreatic movements of the arms and legs.

Following multiple DBS programming sessions (Table 1), including lowering the amplitude and using other contact points, no significant reduction of obsessions and compulsions could be achieved without inducing motor side-effects. The patient additionally suffered from mood swings. Despite continuation of pharmacotherapy and additional psychotherapeutic interventions, including intensive day treatment and behavioural therapy, the patient's quality of life remained very poor, and the patient required further hospital admissions due to increased suicidal thoughts and an impulsively attempted suicide by auto-intoxication.

Due to the programming limitations and the fact that any therapeutic benefit was accompanied by intolerable motor side-effects, we decided to re-operate and target the VC/VS region. In April 2014, at age 51, the patient underwent stereotactic reimplantation of the electrodes with the deepest electrode contact point in the area of the NAc (Model 3387 DBS lead; Medtronic, MN, USA). For final target coordinates see 
Table 1. Postoperative imaging showed adequate placement of the electrodes at the planned target (Figure 1). After programming and additional psychotherapeutic therapy, therapeutic effect was achieved without inducing serious side-effects. For stimulation parameters see Table 1. One year postoperatively, the patient's Y-BOCS score was 29 , and two years postoperatively 17 , a reduction of $50 \%$ compared to baseline. At present, the patient reports a stabilisation of daily life routines and improved quality of life.

Table 1. Patient characteristics and stimulation settings

\begin{tabular}{|c|c|c|}
\hline \multicolumn{3}{|l|}{ Medication used before surgery ${ }^{*}$} \\
\hline & Clomipramine & Haloperidol \\
\hline & Sertraline & Oxazepam \\
\hline & Citalopram & Fluoxetine \\
\hline & Mirtazapine & Venlafaxine \\
\hline & Paroxetine & Aripiprazol \\
\hline Target coordinates $^{\dagger}$ & STN & VC/VS \\
\hline $\mathrm{X}$ & 10 & 7 \\
\hline Y & 0 & $12.8(\mathrm{~L}) / 12.7(\mathrm{R})$ \\
\hline Z & -4 & -3 \\
\hline Stimulator settings & Left & Right \\
\hline \multirow[t]{6}{*}{ Tested STN-DBS $\ddagger$ settings } & 1- C+, $90 \mu \mathrm{s}, 130 \mathrm{~Hz}$ & $9-\mathrm{C}+, 90 \mu \mathrm{s}, 130 \mathrm{~Hz}$ \\
\hline & 2- C+, $90 \mu \mathrm{s}, 130 \mathrm{~Hz}$ & $10-\mathrm{C}+, 90 \mu \mathrm{s}, 130 \mathrm{~Hz}$ \\
\hline & 3- $\mathrm{C}+, 90 \mu \mathrm{s}, 130 \mathrm{~Hz}$ & $11-\mathrm{C}+, 90 \mu \mathrm{s}, 130 \mathrm{~Hz}$ \\
\hline & 1- $2+, 90 \mu \mathrm{s}, 130 \mathrm{~Hz}$ & $9-10+, 90 \mu \mathrm{s}, 130 \mathrm{~Hz}$ \\
\hline & 2- $\mathrm{C}+, 90 \mu \mathrm{s}, 130 \mathrm{~Hz}$ & $9-\mathrm{C}+, 90 \mu \mathrm{s}, 130 \mathrm{~Hz}$ \\
\hline & $0-\mathrm{C}+, 90 \mu \mathrm{s}, 130 \mathrm{~Hz}$ & 8- $\mathrm{C}+90 \mu \mathrm{s}, 130 \mathrm{~Hz}$ \\
\hline \multirow[t]{2}{*}{ Final VC/VS DBS settings } & 1- 2- 3- C+, 3.4 V, $90 \mu \mathrm{s}$ & 9- $10-11-\mathrm{C}+, 3.4 \mathrm{~V}, 90 \mu \mathrm{s}$, \\
\hline & $130 \mathrm{~Hz}$ & $130 \mathrm{~Hz}$ \\
\hline \multicolumn{3}{|l|}{ Y-BOCS } \\
\hline Preoperative & & 34 \\
\hline Postoperative VC/VS 1 year FU & & 29 \\
\hline Postoperative VC/VS 2 year FU & & 17 \\
\hline \multicolumn{3}{|c|}{$\begin{array}{l}\text { *Medication used before surgery refers to all the different pharmacological trials conducted since the patient } \\
\text { was first treated until the first surgery. Adequate doses of these drugs were maintained for at least } 12 \text { weeks. } \\
\text { † Distance from mid-anterior/posterior commissure in millimeter } \\
\text { ₹ Voltages were slowly increased with every setting. Dyskinesias occurred at voltages between } 1.5 \text { and } \\
2.1 \mathrm{~V} \text {. } \\
\text { Abbreviations: STN = subthalamic nucleus, DBS = deep brain stimulation, VC/VS = ventral capsule/ventral } \\
\text { striatum, Y-BOCS = Yale-Brown Obsessive Compulsive Scale, FU = follow up. }\end{array}$} \\
\hline
\end{tabular}




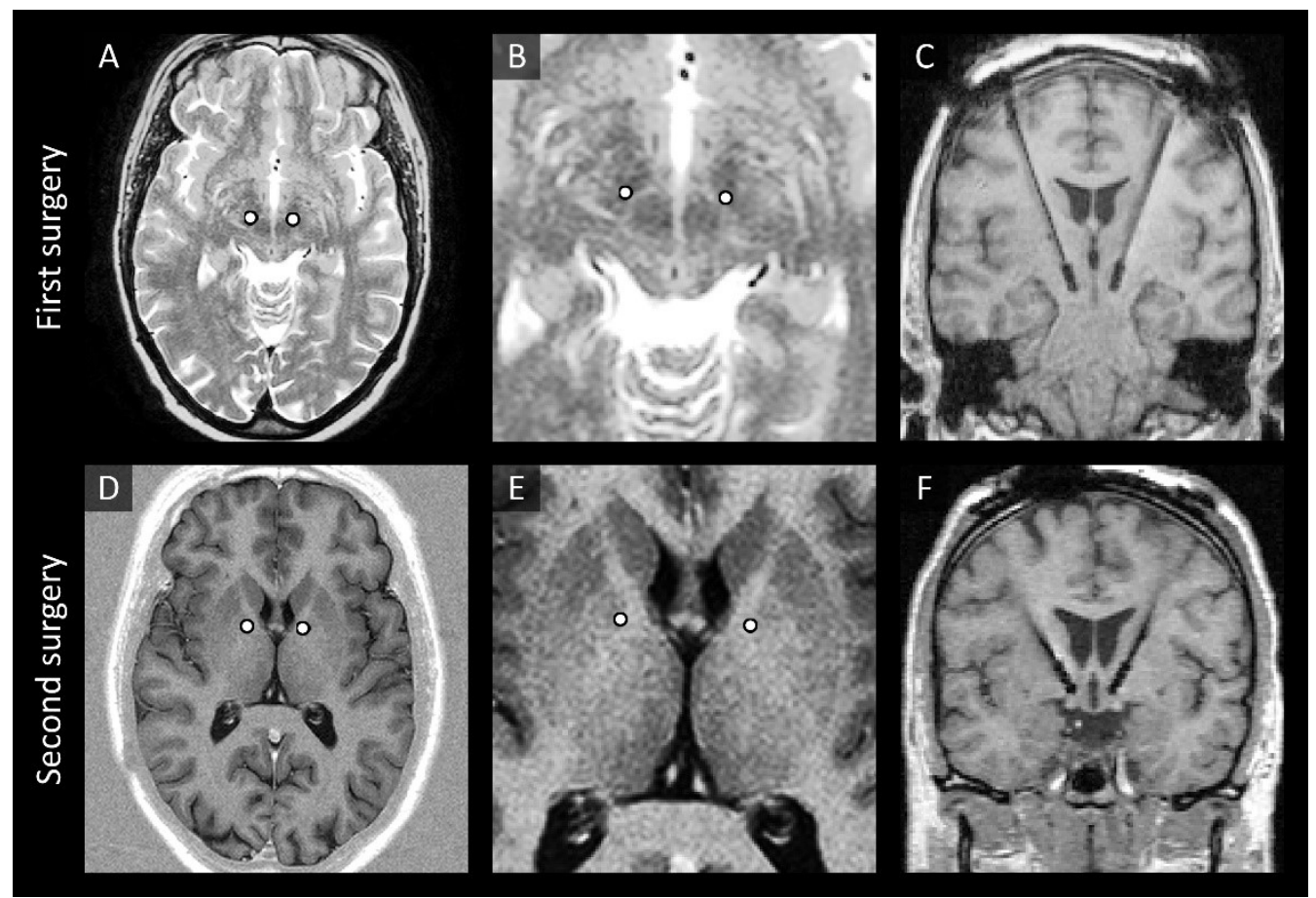

Figure 1. Postoperative location of the electrodes after bilateral anteromedial STN-DBS (first surgery; $A, B, C$ ) and bilateral VC/VS DBS (second surgery; D, E, F). Axial preoperative MR images (A and D) and their enlargements ( $B$ and $E$ ). The location of the active contacts in the preoperative images was computed by rigidly registering the postoperative images and their coordinates of the active contacts to the preoperative images with FSL's FLIRT algorithm ${ }^{24}$. C,F). Postoperative coronal MR images showing the electrodes and contacts.

Abbreviations: STN = subthalamic nucleus, $\mathrm{DBS}=$ deep brain stimulation, $\mathrm{VC} / \mathrm{VS}=$ ventral capsule/ventral striatum, MR = magnetic resonance, FLIRT = FSL's Linear Image Registration Tool.

\section{DISCUSSION}

This case illustrates that DBS of the anteromedial portion of the STN in OCD can cause persistent motor side-effects that resemble chorea and ballism. Choreatic movements and (contralateral) hemiballismus have previously been associated with STN dysfunction ${ }^{7}$, and have been reported following STN-DBS in Parkinson's disease (PD), where typically the dorsolateral sensorimotor portion of the STN is stimulated ${ }^{8-11}$. Although these side-effects are usually transient in $\mathrm{PD}^{11}$ they can make DBS programming difficult ${ }^{8,9}$. Moreover, dyskinetic movements as side-effect of STN-DBS in OCD have been reported in two patients in the STOC-study (French Stimulation dans le Trouble Obsessionnel Compulsif Study Group) in the first month following stimulation. However, these resolved spontaneously or promptly following the adjustment of the stimulation settings ${ }^{12}$. As far as we are aware, such severe and 
persistent motor side-effects that interfere with DBS programming have not yet been described for anteromedial STN-DBS.

The underlying mechanisms and pathophysiology of DBS-induced motor side-effects are not well understood. For the emergence of such symptoms following STN-DBS in PD several disease-related risk factors have been identified, including severe dyskinesias preoperatively and young onset $\mathrm{PD}^{8}$. Additionally, STN-DBS has been demonstrated to increase extracellular striatal dopamine (DA) metabolites in animal models of $P D^{13}$. Whereas $P D$ is characterised by a progressive degeneration of nigral DA neurons, one can speculate that STN-DBS may result in an increase in striatal DA release and subsequently in hyperdopaminergic behaviours such as dyskinesia in an OCD patient with an intact DA system. This is further supported by the finding that the increase in striatal DA metabolites following STN-DBS in naïve rats was accompanied with transitory involuntary movements ${ }^{14}$.

Moreover, dyskinesias following STN-DBS in PD have been associated with contact location within the dorsolateral portion of the $\mathrm{STN}^{8},{ }^{10}$. The idea that choreiform or ballistic movements result from stimulation or lesions of specific subdivisions of the STN is supported by a study in which a Gamma-Aminobutyric Acid (GABA) A-receptor agonist was injected into the sensorimotor, associative, and limbic territories of the STN of green monkeys ${ }^{15}$. Except for behavioural changes, no abnormal movements were observed after microinjection in the anteromedial portion of the STN, whereas injection into the dorsolateral and middle portion of the STN resulted in contralateral violent and rhythmic involuntary movements and leg ballismus, respectively ${ }^{15}$.

Despite postoperative imaging showing adequate placement of the electrodes, individual differences do exist with regards to volume and position of the STN ${ }^{16}$. Additionally, a human clinical study using diffusion-weighted tractography indicated a considerable degree of variation across individuals regarding the volume of structural subdivisions of the STN along the limbic, cognitive and motor domains ${ }^{17}$. In light of the individual differences that exist in structural and functional anatomy of STN subdivisions, it is possible that in the case of the patient discussed, although the anteromedial portion of the STN was targeted, this did in fact not constitute the nonmotor portion of the STN. This may have led to the development of motor side-effects, 
either directly by stimulation of the motor portion of the STN or indirectly by current spreading of contact points in close proximity to the motor portion of the STN. Importantly, while the concept of distinct functional subdivisions within the STN is popular in neuroanatomy research ${ }^{18-20}$, it is still a matter of debate and others have suggested convergence ${ }^{21}$ or large overlap and interactions between these functional subdivisions ${ }^{22,} 23$.

Future attempts to manage OCD with STN-DBS should focus in an individualised, patient-specific selection method that can determine the size and location of functional and structural subdivisions of the STN which may be achieved by, for example, preoperative diffusion weighted imaging and tractography and functional magnetic resonance imaging.

\section{CONCLUSION}

We presented a case of persistent bilateral motor side-effects in the lower extremities that limited programming possibilities in a patient who had undergone bilateral anteromedial STN-DBS for severe refractory OCD. Reimplantation of electrodes targeting the VC/VS with the deepest electrode contact point in the area of the NAc successfully overcame this limitation and resulted in adequate therapeutic response. Reimplantation to an alternative target should be considered if stimulation-associated side-effects interfere with adequate programming of the DBS system and this cannot be resolved by adjusting stimulation parameters.

\section{Patient consent}

Written informed consent was obtained from the patient for publication of this case report and any accompanying images. 


\section{REFERENCES}

1. Alonso P, Cuadras D, Gabriels L, et al. Deep Brain Stimulation for Obsessive-Compulsive Disorder: A Meta-Analysis of Treatment Outcome and Predictors of Response. PloS one 2015;10(7):e0133591.

2. Temel $\mathrm{Y}$, Hescham SA, Jahanshahi A, et al. Neuromodulation in psychiatric disorders. International review of neurobiology 2012;107:283-314.

3. Mulders AE, Plantinga BR, Schruers K, et al. Deep brain stimulation of the subthalamic nucleus in obsessive-compulsive disorder: Neuroanatomical and pathophysiological considerations. European neuropsychopharmacology : the journal of the European College of Neuropsychopharmacology 2016;26(12):1909-1919.

4. Mangas M, Moreira R. Deep brain stimulation for obsessive compulsive disorder: A literature review. Journal of Obsessive-Compulsive and Related Disorders 2013;2(4):391-398.

5. KohI S, Schonherr DM, Luigjes J, et al. Deep brain stimulation for treatment-refractory obsessive compulsive disorder: a systematic review. BMC psychiatry 2014;14:214.

6. Goodman WK, Price LH, Rasmussen SA, et al. The Yale-Brown Obsessive Compulsive Scale. I. Development, use, and reliability. Archives of general psychiatry 1989;46(11):1006-1011.

7. Hawley JS, Weiner WJ. Hemiballismus: current concepts and review. Parkinsonism \& related disorders 2012;18(2):125-129.

8. Baizabal-Carvallo JF, Jankovic J. Movement disorders induced by deep brain stimulation. Parkinsonism \& related disorders 2016;25:1-9.

9. Sriram A, Foote KD, Oyama G, Kwak J, Zeilman PR, Okun MS. Brittle Dyskinesia Following STN but not GPi Deep Brain Stimulation. Tremor and other hyperkinetic movements 2014;4:242.

10. Zheng Z, Li Y, Li J, Zhang Y, Zhang X, Zhuang P. Stimulation-induced dyskinesia in the early stage after subthalamic deep brain stimulation. Stereotactic and functional neurosurgery 2010;88(1):2934.

11. Umemura A, Oka Y, Yamamoto K, Okita K, Matsukawa N, Yamada K. Complications of subthalamic nucleus stimulation in Parkinson's disease. Neurologia medico-chirurgica 2011;51(11):749755.

12. Mallet L, Polosan $\mathrm{M}$, Jaafari $\mathrm{N}$, et al. Subthalamic nucleus stimulation in severe obsessivecompulsive disorder. The New England journal of medicine 2008;359(20):2121-2134.

13. Meissner W, Harnack D, Paul G, et al. Deep brain stimulation of subthalamic neurons increases striatal dopamine metabolism and induces contralateral circling in freely moving 6-hydroxydopaminelesioned rats. Neuroscience letters 2002;328(2):105-108.

14. Paul G, Reum T, Meissner W, et al. High frequency stimulation of the subthalamic nucleus influences striatal dopaminergic metabolism in the naive rat. Neuroreport 2000;11(3):441-444.

15. Karachi C, Grabli D, Baup N, et al. Dysfunction of the subthalamic nucleus induces behavioral and movement disorders in monkeys. Movement disorders : official journal of the Movement Disorder Society 2009;24(8):1183-1192.

16. Richter EO, Hoque T, Halliday W, Lozano AM, Saint-Cyr JA. Determining the position and size of the subthalamic nucleus based on magnetic resonance imaging results in patients with advanced Parkinson disease. J Neurosurg 2004;100(3):541-546.

17. Plantinga BR, Temel $\mathrm{Y}$, Duchin $\mathrm{Y}$, et al. Individualized parcellation of the subthalamic nucleus in patients with Parkinson's disease with 7T MRI. Neurolmage 2016.

18. Alexander GE, Crutcher MD. Functional architecture of basal ganglia circuits: neural substrates of parallel processing. Trends in neurosciences 1990;13(7):266-271.

19. Alexander GE, DeLong MR, Strick PL. Parallel organization of functionally segregated circuits linking basal ganglia and cortex. Annual review of neuroscience 1986;9:357-381.

20. Lambert C, Zrinzo L, Nagy Z, et al. Confirmation of functional zones within the human subthalamic nucleus: patterns of connectivity and sub-parcellation using diffusion weighted imaging. Neurolmage 2012;60(1):83-94. 
21. Percheron G, Filion M. Parallel processing in the basal ganglia: up to a point. Trends in neurosciences 1991;14(2):55-59.

22. Joel D, Weiner I. The organization of the basal ganglia-thalamocortical circuits: open interconnected rather than closed segregated. Neuroscience 1994;63(2):363-379.

23. Janssen ML, Temel Y, Delaville C, et al. Cortico-subthalamic inputs from the motor, limbic, and associative areas in normal and dopamine-depleted rats are not fully segregated. Brain structure \& function 2016.

24. Jenkinson $\mathrm{M}$, Smith $\mathrm{S}$. A global optimisation method for robust affine registration of brain images. Medical image analysis 2001;5(2):143-156. 



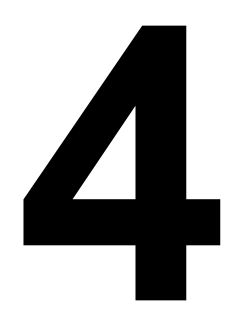

\section{Tryptophan depletion in Parkinson's disease patients treated with deep brain stimulation of the subthalamic nucleus: effects on mood and behaviour}

Anne Mulders, Albert Leentjens, Jos Adam, Anja Moonen, Yasin Temel 


\section{ABSTRACT}

Deep brain stimulation of the subthalamic nucleus (STN-DBS) is an established neurosurgical therapy for Parkinson's disease (PD). However, despite the beneficial motor effects of STN-DBS, some patients experience psychiatric side-effects following surgery, such as depression, mania, and impulse-control disorders. Experimental studies suggest a role of 5-HT in the development of psychiatric side-effects following STN-DBS. In this double-blind, placebo-controlled crossover study we aimed to investigate the role of $5-\mathrm{HT}$ on mood, emotional processing, inhibitory control and motor symptoms in PD patients with STN-DBS by temporarily reducing the level of available $5-\mathrm{HT}$ in the brain by acute tryptophan depletion (ATD). Due to lagging inclusion the study was terminated after including 7 patients. In these 7 patients, we found that 5-HT manipulation by ATD did not lead to dissociable effects on any of the outcome measures, suggesting that psychiatric side-effects following STN-DBS in PD patients cannot be directly linked to a reduction in 5-HT activity. These results, however, should be interpreted with caution given the small sample size. 


\section{INTRODUCTION}

Parkinson's disease (PD) is a progressive neurodegenerative disorder characterised by the degeneration of dopaminergic neurons in the substantia nigra resulting in motor symptoms such as tremor, rigidity and bradykinesia ${ }^{1,2}$. Bilateral high frequency stimulation of the subthalamic nucleus (STN-DBS) is a well-accepted treatment option for $\mathrm{PD}^{3-5}$. STN-DBS results in a significant improvement in motor symptoms and a reduction in dopaminergic medication $3,5,6$. However, there have been conflicting reports on the occurrence of non-motor behavioural changes in a number of patients following STN-DBS, ranging from new onset or worsening of pre-existing behavioural disorders to improvement of neuropsychiatric symptoms. Moreover, psychiatric disorders reported following STN-DBS may show a wide variation ranging from mania and impulse control disorders to and apathy and depression ${ }^{7-9}$. Changes in mood and behaviour can have a large impact on quality of life and could potentially counteract the beneficial effects of STN-DBS on motor symptoms ${ }^{10-12}$. Hence, there is a need for an improved understanding of the mechanisms behind the effects of STN-DBS on mood and behaviour.

The occurrence of depression and/or apathy after STN-DBS, albeit variable, has been associated with a number of causes: post-operative withdrawal of dopaminergic medication, current spreading to the limbic part of the STN, individual variations in the neurodegenerative process, (underestimation of) pre-existing depressive symptoms, and mal-adaptation to the new physical and psychosocial situation ${ }^{7,9,13-15}$. Experimental studies provided evidence that an interaction between the STN and midbrain serotonin (5-HT) system may also be involved in the psychiatric side-effects following STN-DBS. It has been reported that STN-DBS reduced extracellular 5-HT, as well as the firing rate of serotonergic neurons of the dorsal raphe nucleus in a control and in a 6-hydroxydopamine rat model of PD, suggesting a reduction in serotonergic function ${ }^{16-18}$. These effects were both region- and neuron specific. Moreover, STN stimulation was found to evoke depressive-like behaviour, that could be reversed by a 5-HT selective antidepressant ${ }^{17}$. A dysfunctional $5-\mathrm{HT}$ system is known for its role in mood symptoms. Although progressive degeneration of dopamine neurons is regarded the pathological hallmark of $\mathrm{PD}$, it is well established that the $5-\mathrm{HT}$ system is also affected in the PD brain ${ }^{13,19,20}$. A decreased level of 5-hydroxyindole acid (5-HIAA) in cerebrospinal fluid $(\mathrm{CSF})^{21}$, a reduction of post-mortem $5-\mathrm{HT}$ brain levels ${ }^{22,23}$, as well 
as a reduction in 5-HT transporter density in multiple brain regions in the brain of PD patients have been reported 24,25 . Moreover, around $40 \%$ of patients with PD experience depressive symptoms, which is higher than age-matched controls ${ }^{26}$. Given these findings, we suggest that PD patients have a 5-HT vulnerability that, in combination with a decrease in 5-HT transmission elicited by STN stimulation, may increase susceptibility to develop depression and other psychiatric problems following STN-DBS 27 .

The purpose of the present study was to investigate the role of $5-\mathrm{HT}$ on mood and behaviour in PD patients with STN-DBS by temporarily reducing the level of available 5-HT in the brain by means of tryptophan (TRP) depletion. We hypothesised that acute TRP depletion (ATD) in combination with STN-DBS might lead to lower mood, and other behavioural changes such as reduced inhibitory control and deficits in emotional processing.

\section{METHODS}

\section{Subjects}

Seven patients with idiopathic PD who underwent bilateral STN-DBS were enrolled in this study. Patients were recruited from the Movement Disorders Clinic of Maastricht University Medical Centre (MUMC+). Patients were selected for STN stimulation if they had clinical findings consistent with idiopathic PD and severe parkinsonian motor symptoms, on-off fluctuations or dyskinesias that affected their quality of life.

Prior to DBS surgery, all subjects underwent intensive neurological and neuropsychological testing. Exclusion criteria for the surgery were atypical PD, significant atrophy, multiple white-matter lesions or other focal brain abnormalities as observed on magnetic resonance imaging (MRI), dementia, psychosis, Hoehn and Yahr (HY) stage 5 at the best moment of the day, and general contraindications for surgery such as severe hypertension, cardiac or other medical problems. To be eligible for participation in this study, patients had to be mentally competent and receive stable DBS treatment. Patients with neurodegenerative disorders other than PD were excluded. The following additional exclusion criteria were applied: current psychiatric symptomatology, defined by a score $>16$ on the Hamilton Depression Rating Scale $(\mathrm{HAMD})^{68}$ or a score $>2$ on any of the MDS-UPDRS section I items ${ }^{28}$, current 
malignancy or infection, history of stroke or head injury, current use of psychoactive medication, specifically antidepressants and antipsychotics (a stable dose of benzodiazepines were allowed); and, clinically relevant cognitive deterioration operationalised as a score of $<24$ on the Mini Mental State Examination $\left(\right.$ MMSE) ${ }^{29}$.

In order to achieve a representative study sample, participants were included irrespective of their disease stage, disease duration or their current antiparkinsonian medication, provided a stable situation was present. The study was approved by the local Medical Ethics Committee (METC azM/UM, Maastricht). All patients gave their written informed consent prior to participation and received a financial compensation of 100 euro for participating in the study.

\section{Design}

The experiment involved a randomised, double-blind, placebo-controlled, crossover study. The intervention consisted of a TRP depleted mixture to achieve ATD or a placebo mixture, with a wash-out period of minimal one week to exclude carry-over effects. During both test days, participants were tested in DBS ON and DBS OFF condition.

\section{Intervention}

ATD was accomplished by administering a TRP-low collagen-protein mixture (SolugelP, PB Gelatins, Belgium) and was similar as described by ${ }^{30}$. See table 1 for the quantities of amino-acids (AAs). The drink was prepared by mixing $200 \mathrm{ml}$ of cold water to $100 \mathrm{gr}$ of gelatin mixture. The placebo mixture was identical in composition, but 1.21 gr L-TRP (Sigma, Zwijndrecht, the Netherlands) was added, in accordance with previous studies ${ }^{31,32}$. To avoid an unpleasant taste, the mixture was flavoured with two table spoons of protein-free chocolate syrup and participants were offered a spearmint gum after consumption ${ }^{33}$. Blood samples were drawn by venipuncture (Heparin tubes, $4 \mathrm{ml}$ ) before and 3 hours after ingestion of the amino-acid mixture, to establish that TRP levels were adequately reduced. Samples were analysed for total plasma TRP levels and TRP / $\Sigma L N A A$ ratio ( $\Sigma L N A A$; i.e. tyrosine, phenylalanine, leucine, isoleucine and valine). 
Table 1. Amino-acid composition (in grams) of $100 \mathrm{gr}$ protein (Solugel-P) in $200 \mathrm{ml}$ tap water

\begin{tabular}{lclc}
\hline Amino-acid & gr/100 gr & Amino-acid & gr/100 gr \\
\hline Aspartic acid + asparagines & 4.5 & Methionine & 0.9 \\
Glutamic acid + glutamate & 10.0 & Cysteine & 0.0 \\
Hydroxyproline & 12.3 & Isoleucine & 1.2 \\
Serine & 3.4 & Hydroxylysine & 1.5 \\
Histidine & 0.9 & Phenylaline & 1.6 \\
Threonine & 1.9 & Lysine & 3.3 \\
Alanine & 8.4 & Proline & 13.7 \\
Tyrosine & 0.6 & Valine & 2.2 \\
Arginine & 7.7 & Tryptophan & 0.0 \\
\hline Taken from the nutritional datasheet Solugel, edition December 28, 2007 (PB Gelatins, & \\
Tessenderlo, Belgium) & \multicolumn{3}{l}{} \\
\hline
\end{tabular}

\section{Procedure}

On the test days, participants arrived at 9.00 a.m., after an overnight fast. Participants maintained their optimal doses of medication during testing. After informed consent was given, the HAMD and MMSE were obtained to characterise the population and to rule out clinical relevant depressive symptoms and cognitive decline, respectively. Furthermore, demographic and disease-related characteristics were obtained, including disease duration, age at onset, side of onset, as well as current anxiety levels by means of the Parkinson Anxiety Scale ${ }^{34}$. All participants were staged according to the HY staging system ${ }^{35}$. On both test days a baseline measurement was obtained. Then, after a baseline blood sample was taken, they received the amino-acid mixture, which had to be consumed within 15 minutes. The interventions were administered under double-blind conditions. During the ensuing 3 hours, the participants were offered a TRP-low and protein-low lunch. They were asked to minimise their activity, but they were allowed to take a walk in the hospital. Approximately 3.5 hours after the intervention, the second assessment (including blood sample) was conducted, which consisted of the same measures as the baseline assessment. Thereafter, the DBS stimulator was turned off and there was a 15-minute break to ensure the participant adapted to the condition and the motor effects of stimulation changes became visible. A shorter version of the test protocol was conducted, after which the DBS was switched back on. After completion of the testing and re-establishment of normal stimulation parameters participants were offered a high-protein snack and were free to go home. The order of the task presentation can be found in table 2 . 
Table 2. Order of task presentation and time points for administration

\begin{tabular}{|c|c|c|c|c|}
\hline & Instrument & Baseline & DBS ON & $\begin{array}{l}\text { DBS } \\
\text { OFF }\end{array}$ \\
\hline Time (h) & & 0 & 3.5 & 4.5 \\
\hline Depression & Hamilton Depression Rating Scale ${ }^{68}$ & $\mathrm{X}$ & & \\
\hline Anxiety* & Parkinson Anxiety Scale ${ }^{34}$ & $x$ & & \\
\hline Cognitive status* & Mini Mental State Examination 29 & $x$ & & \\
\hline Mood & Profile of Mood States ${ }^{36}$ & $\mathrm{x}$ & $\mathrm{X}$ & $x$ \\
\hline Motor symptoms & $\begin{array}{l}\text { MDS-UPDRS part III28, } \\
\text { Hoehn \& Yahr staging }\end{array}$ & $x$ & $x$ & $x$ \\
\hline Inhibitory control & Anticue reaction time test ${ }^{38-40}$ & $\mathrm{X}$ & $\mathrm{x}$ & \\
\hline $\begin{array}{l}\text { Emotional } \\
\text { processing }\end{array}$ & Informative Affective Picture System ${ }^{37}$ & $x$ & $x$ & \\
\hline
\end{tabular}

\section{Outcome measures}

\section{Mood: Profile of Mood States}

The abbreviated Dutch version of the Profile of Mood States (POMS) questionnaire was used to assess self-reported $\operatorname{mood}^{36}$. This version assesses five different qualities of mood (sadness, tension, anger, vigour, and fatigue) and consists of 32 adjectives describing feelings and moods. Participants were instructed to indicate how well the adjective reflects their current mood on a 5 Point Likert Scale ranging from 0 (not at all) to 4 (extremely). The POMS was administered 3 times during a test day: once at baseline, once 3.5 hours after ingestion of the amino-acid mixture (placebo or ATD) in DBS ON condition, and once 4.5 hours after ingestion of the amino-acid mixture in DBS OFF condition. The total POMS score was the primary outcome measure of this study. The POMS subscales were considered secondary outcome measures.

\section{Motor symptoms}

The Movement Disorder Society-Unified Parkinson's Disease Rating Scale (MDSUPDRS) is a widely used clinical scale rating several aspects of $P D^{28}$. The MDSUPDRS consists of four parts, namely, I: Non-motor Experiences of Daily Living; II: Motor Experiences of Daily Living; III: Motor Examination; IV: Motor Complications. In the present study, part III was used to measure motor symptoms, which was administered 3 times during a test day: at baseline, 3.5 hours after ingestion of the amino-acid mixture (placebo or ATD) in DBS ON condition, and 4.5 hours after ingestion in DBS OFF condition. 


\section{Emotional processing}

To test emotional processing, 180 colour pictures were selected from the International Affective Picture System (IAPS) ${ }^{37}$ and, based on their normative valance and arousal ratings, divided into four parallel versions of 45 pictures each. 'Valence' refers to the nature of emotional activation: positive, neutral or negative. 'Arousal' refers to the intensity of emotional activation, ranging from low to high arousal ${ }^{37}$. The different versions were balanced with respect to their normative valance and arousal ratings, as well as with regard to stimulus complexity, colour, and the presence of animals and human beings. Selected positive pictures included couples, food, sport activities, babies, etc. Neutral pictures included plants, furniture, buildings, etc. Negative pictures included human violence, threatening animals, mutilations, etc. There were no significant differences in mean total valence and arousal levels between the different versions, and there were no significant differences for each stimuli category (negative, positive, neutral) between the different versions. For the mean valence and arousal ratings for each version, see Supplementary table 1. For a list of the selected IAPS pictures, see Supplementary table 2. Participants were tested twice during a test day: at baseline, and approximately 3.5 hours after ingestion of the amino-acid mixture (placebo or ATD) in DBS ON condition.

\section{Inhibitory control: anticue reaction time test}

A computerised anticue reaction time test was used to test inhibitory control ${ }^{38-40}$. The test consisted of a row of four empty boxes ( $35 \mathrm{~mm} \times 35 \mathrm{~mm}$ ), separated by $25 \mathrm{~mm}$ (between adjacent sides), which were continuously visible as squares in orange-brown outline on a black background screen (Figure 1). At the start of each trial, a visual warning signal was presented as a small red square $(10 \mathrm{~mm} \times 10 \mathrm{~mm})$ midway between the two inner boxes. The dot flickered three times during an interval of 750 $\mathrm{ms}$, after which it disappeared. After an additional period of $750 \mathrm{~ms}$, either all four boxes turned red (neutral cue) or the two leftmost or the two rightmost boxes turned red (anticue). After a cue-target interval which varied between $100 \mathrm{~ms}, 150 \mathrm{~ms}, 250$ $\mathrm{ms}, 450 \mathrm{~ms}$, and $850 \mathrm{~ms}$, the single target stimulus was presented by colouring one box green. Participants were instructed to indicate the location of the target as quickly and accurately as possible by pressing the corresponding response key. Moreover, they were instructed to take advantage of the anticue by preparing the fingers on the hand opposite to the side of the cue (so that a left-side cue would indicate a right hand 
response, and vice versa). Responding implied pressing one of the four keys with the index and middle fingers of both hands. An incorrect response was accompanied with an error message displayed on the screen. After pressing one of the response keys, the boxes became empty again. The test consisted of 200 trials, divided over four blocks of 50 trials each with a small break of 30 seconds in between ${ }^{39}$. Participants were tested twice during a test day: at baseline, and approximately 3.5 hours after ingestion of the amino-acid mixture (placebo or ATD) in DBS ON condition.
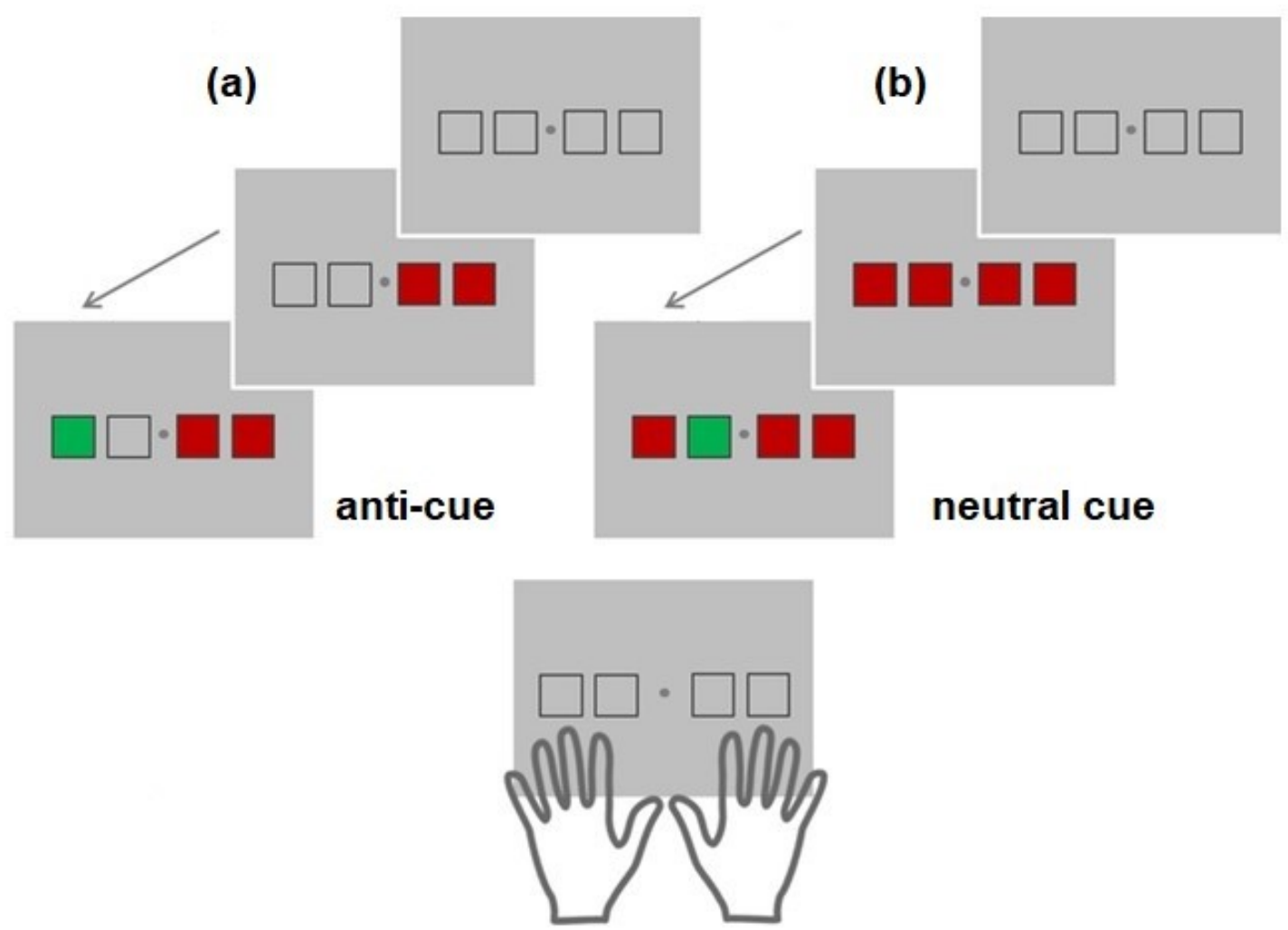

Figure 1. Anticue paradigm.

Four empty boxes are presented. The two squares on the right and left side of the screen correspond to the index and middle finger of the right and left hand, respectively. The visual target was a green square. As soon as one of the four squares turned green, participants had to press the corresponding response key as fast as possible. There were two possible cue types that appeared before the visual target. In the anticue trials (a) the cue was presented on the side opposite of the target. In neutral-cue trials (b) the cue was presented on both sides. Participants were instructed to take advantage of the anticue by preparing the fingers on the opposite hand. The cue-target interval varied between 100, 150, 250, 450 and $850 \mathrm{~ms}$, reflecting the available time to prepare the hand indicated by the cue. (Figure adapted from ${ }^{69}$ ). 


\section{Statistical analysis, power calculation and randomisation}

All analyses were performed using IBM SPSS statistics 24.041. Changes in plasma TRP levels and TRP / ILNAA ratios for both test days were assessed with pairedsample $t$-test comparing baseline blood levels with baseline levels $3 \mathrm{~h}$ after the intake of the amino-acid mixture. Possible differences in baseline measurements were calculated using a 2-sided independent sample $t$-test. Dependent variables of the POMS, the primary outcome measure of this study, and the MDS-UPDRS were assessed by a 2-way analyses of variance (ANOVA) in a repeated measures design, which allowed for assessment of a main effect of "intervention" (ATD vs. placebo), "stimulation" (DBS ON vs. DBS OFF), and their interaction "intervention x stimulation". Dependent variables of the emotional processing task were likewise assessed by a 2way ANOVA repeated measures design, but then looking at the main effect of "time" (baseline vs. $3.5 \mathrm{~h}$ after intake of the amino-acid mixture), the main effect of "intervention" (ATD vs. placebo), and their interaction "time $x$ intervention". For the dependent variables of the anticue reaction time test (reaction time (RT) and percentage of errors), we looked at the main effects of "time", "intervention", "cue-target interval", "cue type" and their interactions. The primary outcome measure was considered significant if $p<0.05$, secondary outcomes effects were considered significant if $p<0.01$, in order to protect against multiple testing errors. Sample size calculation was based on a previous study in which the ATD paradigm was used in PD patients with the POMS as primary outcome measure ${ }^{42}$. A sample size of 30 patients would be adequate for detecting a difference of $1.1 \mathrm{SD}$ with a power of $90 \%$ in each of the four conditions. Randomisation involved a randomised block design of 30 subjects randomised over 5 blocks. The plan was created on 10-12-2015 using the website 'randomization.com (no 3073).

\section{RESULTS}

\section{Procedure}

Due to lagging inclusion the study was terminated after including 7 patients. Of these, three participants were randomised to receive the ATD intervention at the first test day and the placebo mixture on the second day. Four participants were randomised to receive the ATD intervention at the second test day and receive placebo on the first day. All participants tolerated the intervention well and there were no adverse events, regardless of whether they received the ATD or placebo mixture. There were no 
significant differences in baseline plasma TRP and baseline TRP / $\Sigma$ LNAA ratio for the different conditions. Figure 2 shows that a significant reduction of plasma TRP was achieved during the ATD procedure compared to baseline. Three hours after drinking the TRP-free mixture, plasma TRP levels were significantly reduced by $50.7 \%$ (range 39 to $55 \%)$, from 63.3 to $31.3 \mu \mathrm{mol} / / \mathrm{l}(\mathrm{t}(6)=15.906, \mathrm{p}=0.000)$. The TRP $/ \Sigma L N A A$ ratio was reduced by $60.9 \%$ (range 52.9 to $70 \%$ ), from 0.091 to $0.036 \mu \mathrm{mol} / \mathrm{l}(\mathrm{t}(6)=18.761$, $\mathrm{p}=0.000)$. Significant increases in plasma TRP $(86.7 \%$, range 17 to $155 \%, t(6)=-5,792$, $\mathrm{p}=0.001)$ and TRP / $\Sigma$ LNAA ratio $(36.5 \%$, range 16 to $54.55, \mathrm{t}(6)=-7,546, \mathrm{p}=0.000)$ were observed $3 \mathrm{~h}$ following administration of the placebo mixture. The DBS was turned off in all patients. Besides an increase in motor symptoms, there were no adverse motor events, and motor symptoms generally recovered immediately after turning the stimulator back on. In one patient the recovery took approximately 60 minutes.

\section{Demographics and clinical characteristics}

Six male patients and one female patient with an average age of 62.7 years (SD 11.6) were included in this study. The demographic and clinical characteristics of the subjects are summarised in table 3 . At the time of testing, all participants received stable DBS treatment. The time since DBS surgery ranged from 1 to 9 years. Baseline MDS-UPDRS part 3 score at the first visit was 27.14 (SD 12.3).
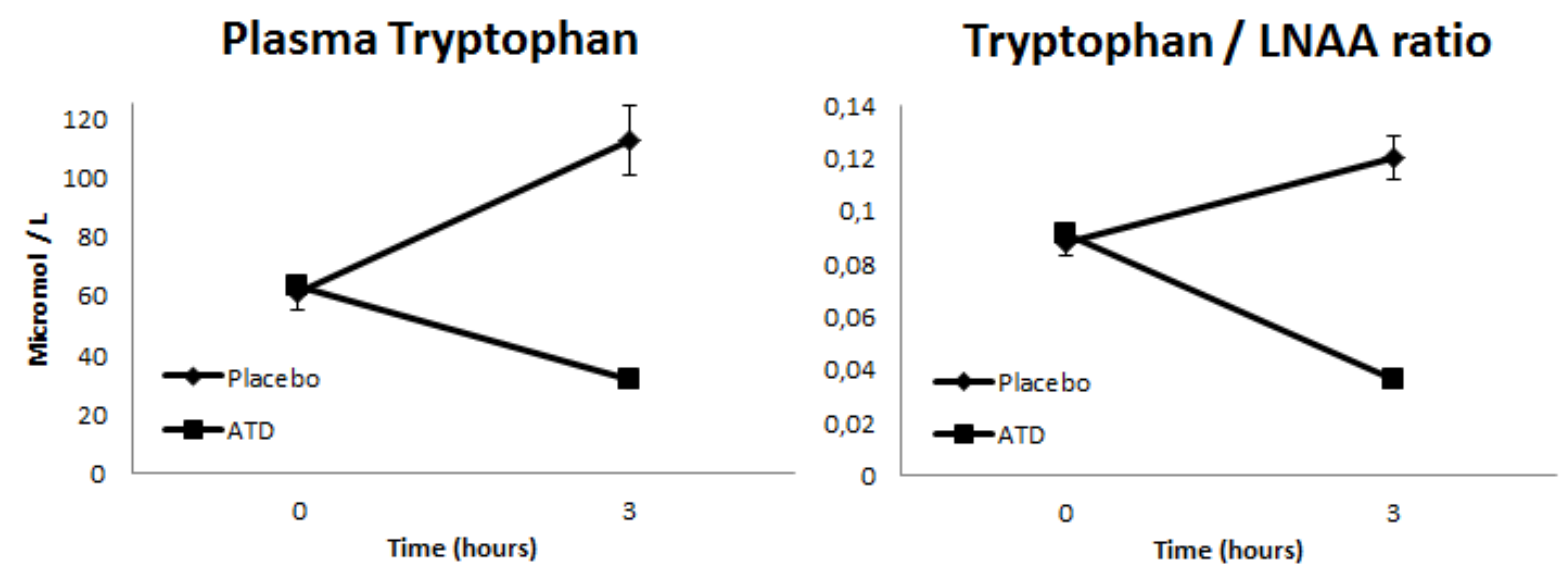

Figure 2. The means and standard errors (SE) of the blood plasma levels of tryptophan ( $\mu \mathrm{mol} / \mathrm{l})$ and the ratio between tryptophan and the other large amino-acids (TRP/LNAA) at baseline (0) and $3 \mathrm{~h}(3)$ after intake of the ATD or placebo mixture. 
Table 3. Demographic and clinical characteristics of the participants

\begin{tabular}{|c|c|c|}
\hline \multicolumn{2}{|c|}{ Characteristics } & $\mathrm{N}=7$ \\
\hline \multicolumn{2}{|l|}{ Age } & $62.7(11.6)$ \\
\hline \multicolumn{2}{|c|}{ Gender (\% male) } & $85.7 \%$ \\
\hline \multicolumn{2}{|c|}{ Education, years } & $11.9(6.4)$ \\
\hline \multicolumn{2}{|c|}{ PD dominant side (\% right) } & $71.4 \%$ \\
\hline \multicolumn{2}{|c|}{ Handedness (\% right) } & $57.1 \%$ \\
\hline \multicolumn{2}{|c|}{ Disease duration, years } & $13.4(4.5)$ \\
\hline \multicolumn{2}{|c|}{ Age at DBS surgery } & $59.6(13.3)$ \\
\hline \multicolumn{2}{|c|}{ DBS, years (median) } & $2.0(2.9 ;$ range 1-9) \\
\hline \multicolumn{2}{|c|}{ MMSE } & 29.1(1.2) \\
\hline \multirow[t]{4}{*}{ PAS } & Total score & $7.4(5.7)$ \\
\hline & Persistent & $3.0(3.2)$ \\
\hline & Episodic & $2.0(1.5)$ \\
\hline & Avoidance & $2.43(2.1)$ \\
\hline \multicolumn{2}{|c|}{ LED (mg/day) } & $1026.4(651.9)$ \\
\hline \multicolumn{2}{|c|}{ Hoehn \& Yahr (median) } & $2.0(0.5$; range $2-3)$ \\
\hline
\end{tabular}

\section{Mood}

Figure 3 shows the POMS scores over time in the ATD and placebo condition. On top of the POMS total score and the scores on the POMS subscales, an additional POMS score was added which involves the sum of the scores of the subscales depression, anger and tension. This way, the scale corrects for tiredness, as all patients reported increased fatigue during the course of both test days. There were no significant differences in baseline POMS total scores for the different interventions $(p=0.341)$, or in any of the POMS subscales, see Supplementary Table 2. There was no significant interaction effect and no statistical significant main effect for "intervention" for the POMS total score. Although not statistically significant, the main effect for "stimulation" showed a trend $(F(1,6)=5.93, p=0.051)$, reflecting an increase in total POMS score after the stimulation was switched off. As for the corrected total POMS scores, there were no statistically significant effects for "intervention", "stimulation" or its interaction. No significant "intervention' effect or interaction effects could be found for any of the subscales. However, there was a significant main effect for "stimulation" for the subscales tension $(F(1,6)=14.50, p=0.009)$ and fatigue $(F(1,6)=10.45, p=0.018)$, which 
reflects an increase in tension and fatigue after turning the stimulator off, independent of the intervention ATD or placebo.
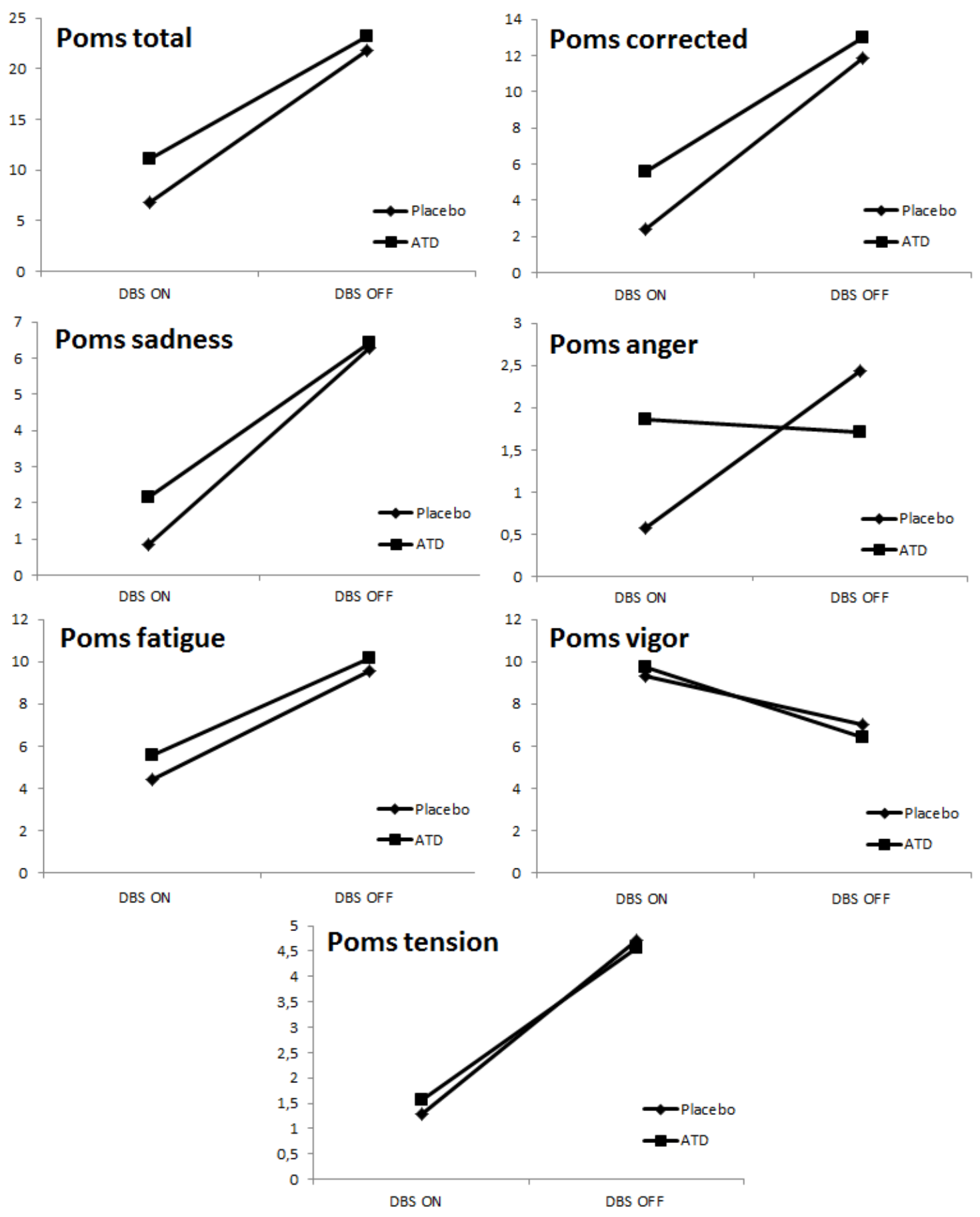

Figure 3. Scores on the POMS in PD patients in the placebo and ATD condition with DBS ON and DBS OFF. 


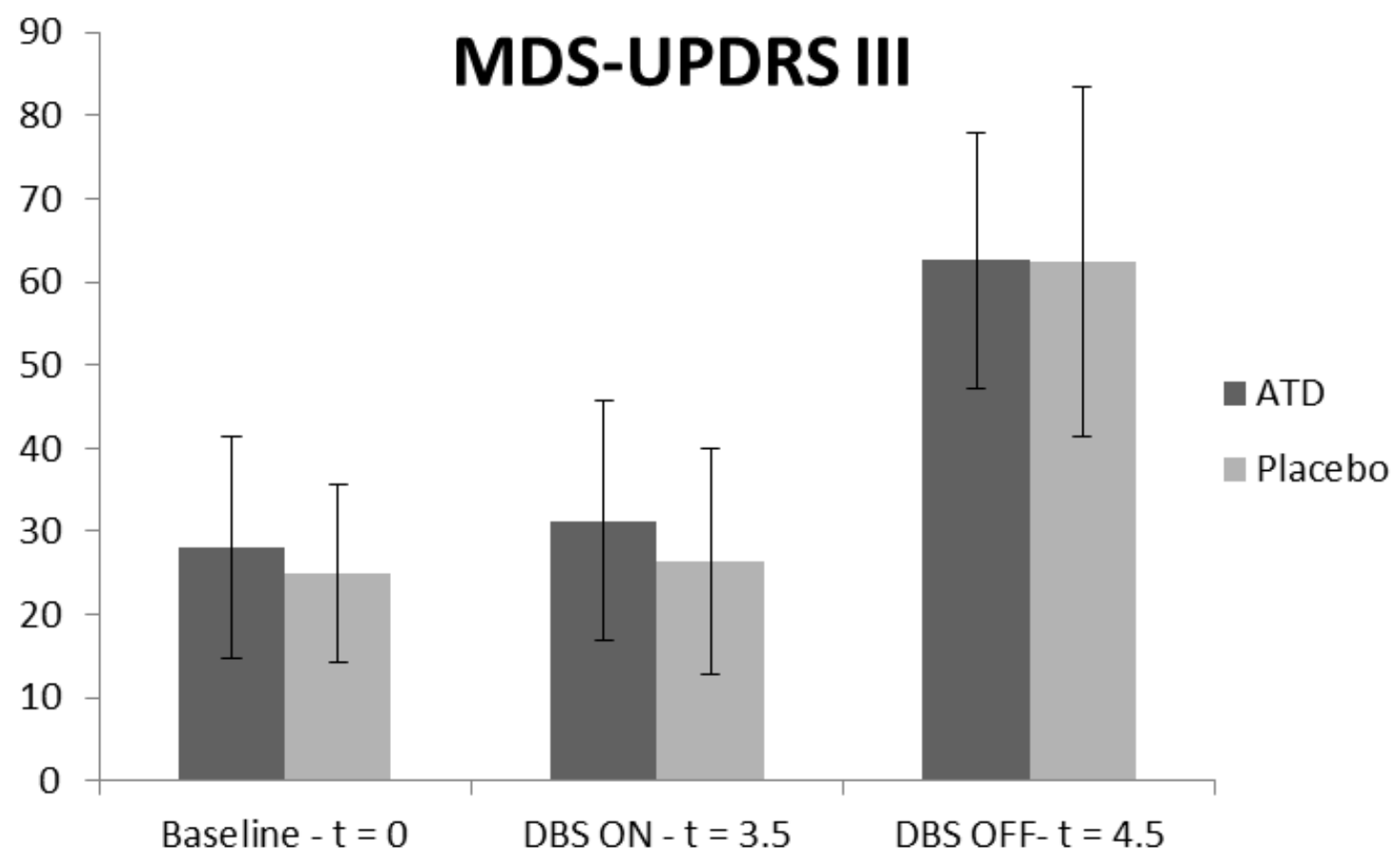

Figure 4. The means and standard deviations on the MDS-UPDRS part III in PD patients at baseline $(\mathrm{t}=0)$ and $3.5 \mathrm{~h}$ in DBS ON ( $\mathrm{t}=3.5)$ and $4.5 \mathrm{~h}$ in DBS OFF ( $\mathrm{t}=4.5)$ after intake of the ATD or placebo mixture.

\section{Motor symptoms}

Figure 4 shows the scores on the MDS-UPDRS part III over time in ATD and placebo condition. No statistically significant effect for "intervention" was observed. The main effect "stimulation" was statistically significant $(F(1,6)=43.9, p=0.001)$, reflecting an increase in MDS-UPDRS part III scores after turning the stimulator off. There was no interaction effect, which indicates that this increase in motor symptoms was independent of the intervention ATD or placebo.

\section{Emotional processing}

For both negative and positive stimuli, as well as neutral stimuli, there were no significant differences in baseline arousal and valence ratings. A statistically significant main effect for "time" was only found for valence ratings of neutral stimuli $(F(1,6)=10.08, p=0.005)$, not for positive or negative stimuli. There was no main effect of "intervention", nor any interaction effects for the valence ratings, regardless of the stimulus type. For arousal ratings, no main effects and no interaction effects were 
found. This means that there was no within-group effect of ATD on emotional processing to negative, positive or neutral stimuli. See table 4 and figure 5 for valence and arousal ratings over time on the IAPS in the placebo and ATD condition.

Table 4. Valence and arousal ratings on the emotional processing task

\begin{tabular}{lccccc}
\hline & \multicolumn{2}{c}{ Placebo } & & \multicolumn{2}{c}{ ATD } \\
\cline { 2 - 3 } \cline { 5 - 6 } Time (h) & Baseline & DBS ON & & Baseline & DBS ON \\
IAPS Valence ratings & 0 & 3.5 & & 0 & 3.5 \\
$\quad$ Negative & $2.51(1.0)$ & $2.21(1.2)$ & & $2.35(1.1)$ & $2.25(0.9)$ \\
Positive & $6.60(0.6)$ & $6.10(0.7)$ & & $6.58(1.4)$ & $6.17(0.9)$ \\
$\quad$ Neutral & $5.04(0.5)$ & $4.53(0.5)$ & & $5.26(0.6)$ & $4.82(0.2)$ \\
IAPS Arousal ratings & & & & \\
$\quad$ Negative & $6.80(1.2)$ & $6.74(0.9)$ & & $6.72(1.3)$ & $6.63(1.31)$ \\
Positive & $5.47(1.2)$ & $6.05(1.0)$ & & $5.40(1.6)$ & $5.98(1.0)$ \\
$\quad$ Neutral & $3.08(1.3)$ & $3.51(1.5)$ & & $3.83(1.0)$ & $4.13(1.2)$ \\
\hline Data are presented as means (SD). ATD = Acute tryptophan depletion, IAPS = International Affective \\
Picture System ${ }^{37}$
\end{tabular}

\section{Inhibitory control}

Reaction time (RT) data as a function of intervention, cue-target interval, and cue type are presented in figure 6 . There was no main effect for "intervention". The main effect of "cue-target interval" was significant, indicating shorter RTs for longer-cue-target intervals ( $M=736 \mathrm{~ms}, 738 \mathrm{~ms}, 701 \mathrm{~ms}, 684 \mathrm{~ms}, 697 \mathrm{~ms}$, respectively $(F(4,24)=12.560$, $p=0.000$ ). However, this was not the case for RTs of cue-target intervals of $150 \mathrm{~ms}$ and $850 \mathrm{~ms}$. No main effect for "cue type" (neutral vs anticue) were found. However, there was a significant "cue type $x$ cue-target interval" interaction $(F(4,24)=7.831, p=0.000)$, which reflected anticue RT costs for the three shortest preparation intervals and anticue RT benefits for the two longest preparation intervals (relative to the neutral cue condition). In other words, anticues hindered RT performance with short preparation intervals ( $\leq 250 \mathrm{~ms}$ ) but facilitated RT performance with longer preparation intervals ( $\geq$ $450 \mathrm{~ms}$ ). This cost-benefit pattern as a function of cue-target interval and cue type was independent of "intervention". There were no other main or interaction effects. The percentage of errors was higher in the placebo condition compared to the ATD condition ( $\mathrm{M}=4.9 \%$ vs. $3.1 \%$, respectively), but this difference was not significant $(F(1,6)=9.615, p=0.021)$. There was a significant main effect of "Cue-target Interval", 
$(F(4,24)=10.162, p=0.000)$, with a significant lower percentage of errors for the longest interval of $850 \mathrm{~ms}$, compared to the shorter intervals of $100 \mathrm{~ms}, 150 \mathrm{~ms}, 450 \mathrm{~ms}$ (M = $1.6 \%, 4.3 \%, 5 \%$, and $5.1 \%$, respectively). No other main or interaction effects were found. Taking together, there was no significant within-group effect of ATD on RT.

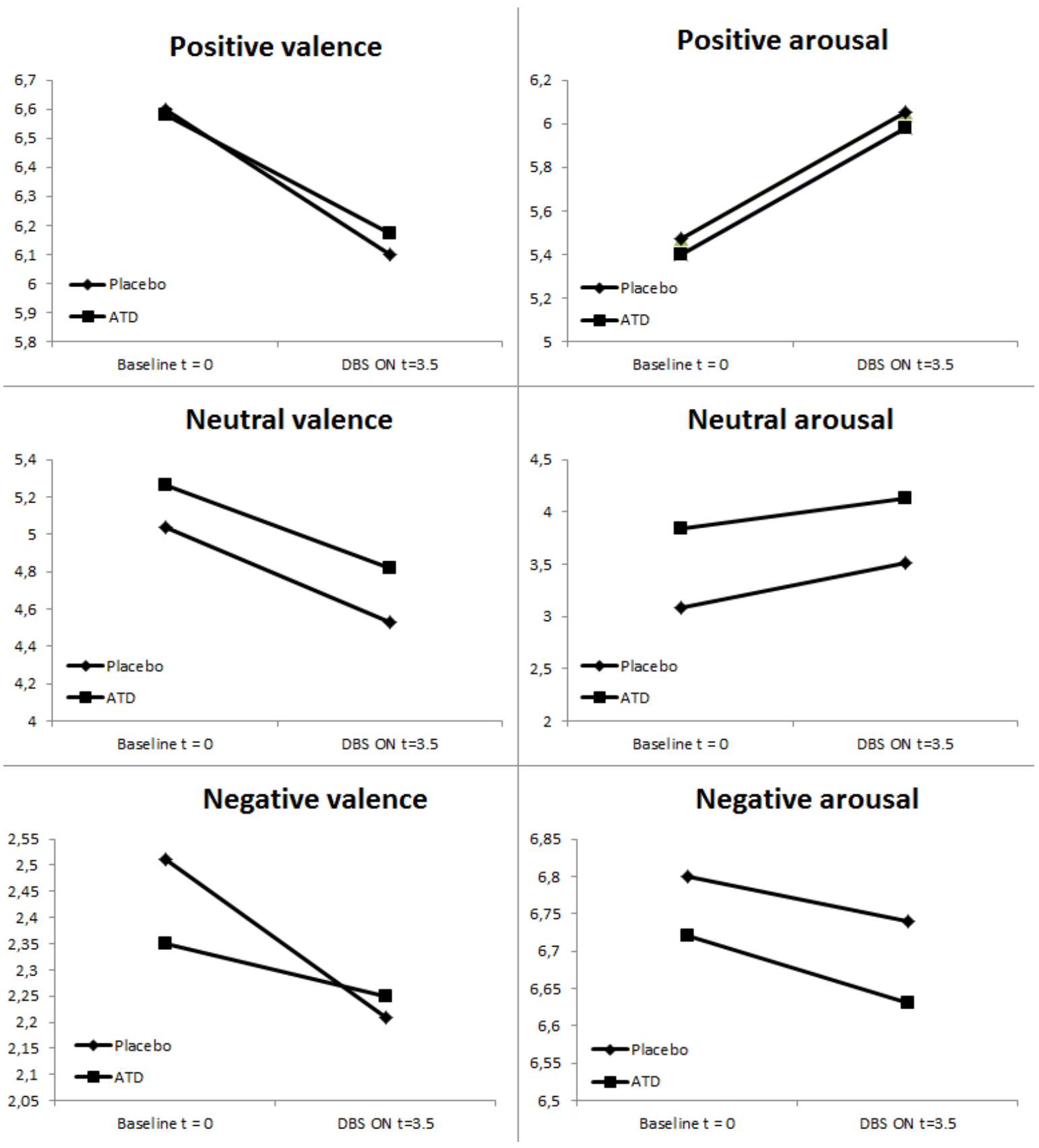

Figure 5. Valence and arousal ratings for positive, neutral and negative stimuli in PD patients in the placebo and ATD condition at baseline $(\mathrm{t}=0)$ and $3.5 \mathrm{~h}(\mathrm{t}=3.5)$ after the start of the intervention in DBS ON. 
(a) Placebo

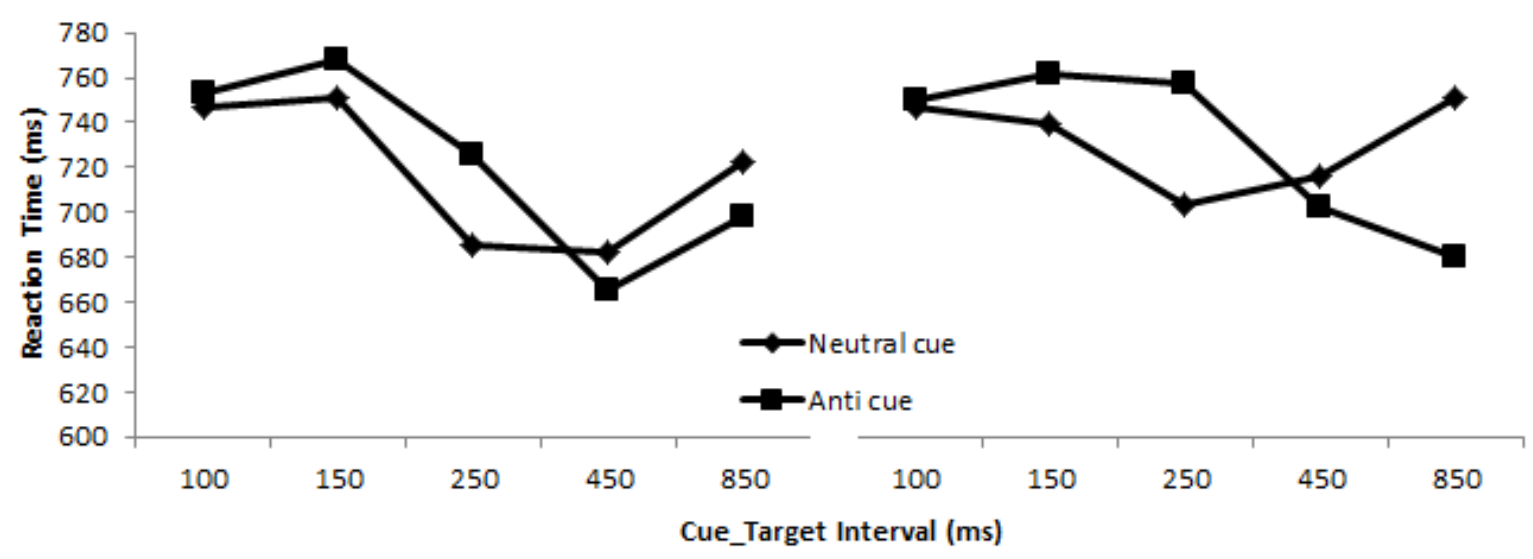

Figure 6. Mean reaction time (ms) of PD patients as a function of intervention ( $a=$ placebo, $b=A T D)$, cuetarget interval, and cue type.

\section{DISCUSSION}

The ATD paradigm has been extensively used to reduce central 5-HT transmission and to study the role of $5-\mathrm{HT}$ in the regulation of diverse motor, cognitive and affective brain functions. The present study aimed to study the effects of ATD on mood and behaviour, including emotional processing, motor functions and inhibitory control in PD patients who received STN-DBS. It was hypothesised that a reduction in 5-HT levels, as accomplished with ATD, in combination with STN-DBS which in turn is hypothesised to lead to a decrease in 5-HT levels in the brain, results in mood lowering effects in PD patients. Moreover, it was hypothesised that ATD together with STN-DBS results in reduced inhibitory control and deficits in emotional processing.

\section{Mood}

There was no differential effect of ATD and the placebo condition on mood scores in both DBS ON and DBS OFF condition. With respect to the ATD intervention, our findings are in line with earlier studies that have used ATD to assess mood changes in PD patients ${ }^{42,43}$. In both studies a healthy control group was included and the lack of an effect of ATD on mood specific to PD patients was interpreted as evidence against the serotonergic hypothesis for depression in $\mathrm{PD}^{42,43}$. Nonetheless, from those findings it can be argued that if 5-HT down regulation is involved in depressive symptoms in PD, a further reduction in 5-HT levels, e.g. by STN-DBS, would be likely to have a 
significant effect on mood $^{43}$. However, in contrast to what was expected, turning the DBS off led to an increase in mood symptoms and these changes were independent of TRP levels.

Several potential explanations for these findings should be considered. First of all, the hypothesis that STN-DBS results in a decrease in central $5-\mathrm{HT}$ levels in the brain is based on animal research and has, af far as we are aware of, not been confirmed in clinical studies. Only one study examined how cerebrospinal fluid (CSF) and plasma catecholomanie levels, including 5-HIAA and 5-HT, were modulated in 11 PD patients following STN-DBS, but no significant changes could be detected ${ }^{44}$. Hence, a reduction in 5-HT levels and an increase in depressive-like behaviour following STN-DBS as seen in experimental studies may not be evident in humans, especially given that animal models cannot faithfully model mood in humans ${ }^{19}$. Another possibility is that the period in which patients were tested with their DBS OFF was too short to accomplish a significant increase in 5-HT levels that could evoke any acute effect on mood. However, we agreed that turning the DBS off for a longer period would be too burdensome for patients and ethically not acceptable. Indeed, being confronted with the physical discomforts elicited by turning the stimulator off resulted in a significant increase in mood symptoms, as was reflected by a trend in POMS total score and a significant increase in the subscale Tension. Interestingly, it has been suggested that any effect of ATD on 5-HT release is presumably greater when participants are in a higher state of arousal ${ }^{45}$. It can be argued whether participants were in a higher state of arousal in the DBS OFF state for the reasons described above, or in a lower state of arousal as a consequence of increased motor symptoms such as akinesia, but nevertheless, we could not find any differential effect on mood in the ATD or placebo condition.

Recently, more emphasis has been placed on the relationship between motor and nonmotor symptoms such as mood problems in PD, as well as to the relationship between fluctuations in non-motor and motor symptoms ${ }^{46}$. In general, research suggests that changes in mood are correlated with motor states in which symptoms such as depression are more present in the off-state ${ }^{47,48}$. The findings in the present study confirm this relationship, and it seems that any effect that ATD might have had on mood was either to subtle to be detected by the POMS or masked by the impact of the 
profound increase in motor symptoms after turning the stimulator off. It should be noted that all participants in this study were tested while continuing to take their regular dopaminergic medication, including levodopa which may interfere with the serotonergic neurotransmitter system ${ }^{49}$. It is, however, unlikely that this influenced the effect of ATD on mood, as an earlier study that excluded PD patients with levodopa medication reported similar results ${ }^{42}$. Taken together, based on the findings of this study, it seems that the vulnerability for depression in PD in general and following STN-DBS cannot be explained by changes in overall serotonergic activity.

\section{Emotional processing}

Apart from evaluating the effect of ATD on self-reported mood states, the effect of ATD on emotional processing was explored. Emotional processing refers to both conscious and unconscious processes of recognising, experiencing, and expressing emotions $^{50,51}$. Previous research that used TRP depletion to study changes in emotional processing has found effects independently from changes in mood ${ }^{52}$. Indeed, although depression is associated with disturbed emotional processing, the latter can also be present in PD patients in the absence of clinical disturbances in $\operatorname{mood}^{50,53}$. In the present study, ATD did not have any effect on the way participants rated neutral, positive or negative stimuli in terms of arousal and valence. These findings should be interpreted in the light of the neurobiological correlates of emotional processing in PD. Emotional and affective processes are influenced by a limbic cortexbasal ganglia network, consisting of areas such as the amygdala, ventral striatum, ventral anterior cingulate cortex, orbitofrontal cortex and ventrolateral prefrontal cortex ${ }^{50,53}$, as well as the ventral part of the STN ${ }^{54,55}$. This network largely depends on dopaminergic projections and it has been proposed that progressive degeneration of dopaminergic pathways as seen in PD can effect the functionality of those regions and lead to deficits in emotional processing. On the other hand, despite disturbed functionality of this network, there is evidence that more dorsal regions of the brain are relatively spared and these regions may serve as a top-down cognitive compensatory mechanism that in turn restores the processing of emotions at the behavioural level, as shown by similar ratings of emotional stimuli in PD patients and healthy controls ${ }^{50,53}$. Findings from clinical studies suggest that STN-DBS leads to impaired emotional processing in $\mathrm{PD}^{56-58}$, and studies that have used DBS to study the functional role of the STN in emotional processing provide evidence that emotional processing is related 
to changes in STN neural activity $55,59,60$. Moreover, it has been hypothesised that the STN plays a more general role in emotional processing, irrespective of the emotion component, type of emotion or stimulus valence (positive or negative) ${ }^{61}$, but the underlying mechanisms of changes in emotional processing following STN-DBS remain to be elucidated. Although the findings from the present study need to be interpreted with cautioun as participants were only tested in DBS ON condition in the absence of a healthy control group, it seems that impaired emotional processing following STN-DBS is not modulated by serotonergic neurotransmission.

\section{Motor symptoms}

There was no differential effect of ATD and the placebo condition on motor symptoms in both DBS ON and DBS OFF state. These findings are in accordance with the findings from Scholtissen et al. (2006) who hypothesised that the UPDRS, which is comparable to the MDS-UPDRS used in the present study, is likely not sensitive enough to detect small changes in motor performance due to changes in TRP levels ${ }^{19}$. Indeed, motor symptoms increased significantly after turning the stimulator off, but this was again irrespective of TRP levels.

\section{Inhibitory control}

The anticue reaction time test was used to study inhibitory control. Inhibitory control, also known as response inhibition, refers to the ability to actively inhibit or delay a dominant response to achieve a goal, and is considered to be a central component of executive functioning ${ }^{62,63}$. A decrease in $5-\mathrm{HT}$ neurotransmission has been associated with diminished inhibitory control, which in turn can lead to impulsive behaviour ${ }^{64}$. Moreover, the STN is believed to play a role in inhibitory control and there is evidence that STN-DBS can lead to altered response inhibition when faced with conflict 62,63 . Importantly, inhibitory control can be reactive- that is, externally stimulus-driven, or proactive- that is, goal-driven and prior to the occurrence of cognitively demanding events ${ }^{65}$. The anticue reaction time test provides the opportunity to evaluate top-down proactive inhibitory control in the face of conflict, as it provides a predictive cue that is incongruent to the response location ${ }^{39}$. In the present study, there was a significant "cue type x cue-target interval" interaction, which reflected anticue RT costs for shorter preparation intervals and RT benefits for longer preparation intervals, indicating that applying inhibition takes time. This finding is in line with other studies that examined 
anticue reaction time performance in healthy participants ${ }^{39,66}$, as well as in PD patients who received STN-DBS ${ }^{38}$. In the latter study, 4 PD patients were tested in different condtions (DBS - medication on, DBS on - mediation off, DBS off - medication on, DBS - medication off). Except for the DBS off - medication off condition, similar cross-over patterns between anticue costs and benefits were observed ${ }^{38}$. That is, when there was no DBS and no medication, there we no RT benefits, indicating the absence of effective response inhibition. Interestingly, in the present study there were no differences in RT or percentage or errors between the ATD and placebo condition, nor were there any interaction effects between the intervention (ATD or placebo), cue-target interval (100 ms, $150 \mathrm{~ms}, 250 \mathrm{~ms}, 450 \mathrm{~ms}, 850 \mathrm{~ms}$ ) or cue type (anticue or neutral cue), which means that patients were still able to create RT benefits for longer preparation intervals and had similar error rates when central 5-HT activity was reduced. These findings seem to suggest that the top-down control needed in the anti-cue task, which reflects both inhibition and facilitation processes ${ }^{66}$, relies upon other mechanisms than $5-\mathrm{HT}$ neurotransmission.

\section{Early termination of the study and limitations}

Due to lagging inclusion the study was terminated prematurely. The recruitment difficulties were the result of a combination of factors, including strict in- and exclusion criteria because of the vulnerability of the research population, and the fact that many patients were unable to come to the test side for two full days, as they often relied upon caregivers for transport and further support. Moreover, many patients were reluctant to have their stimulator turned off. Importantly, for the patients that did participate, we observed effects in opposite direction of what was hypothesised. The return of motor symptoms as a consequence of switching the stimulator off turned out to be emotionally overwhelming for participants, which in turn masked possible subtle differential effects that ATD might have had on mood. Overall, it was concluded that the study was not feasible and continuation would be ethically not acceptable as any further results that might have been found would not be in proportion to the difficulties and risks imposed on the research participants. Due to the remaining small sample size, results should be interpreted with caution. This is especially the case for outcome measures that are less sensitive such as the POMS and emotional processing test, in which subtle changes caused by ATD might not have been visible due to underpowerment. On the other hand, the anticue reaction time test is a very sensitive 
test that has been proven to be senstive enough to detect differential responses even with a lower number of subjects ${ }^{38}$.

Another limitation of the study is that only one blood sample was taken to establish that TRP levels were adequately reduced. Based on results from other studies who have used the ATD paradigm to study changes in mood, it has been suggested that the decline in plasma TRP has to be around $60 \%$ or greater to see any effect on mood 45,67 . Although we did not reach a reduction of $60 \%$ in this study, the time between consuming the mixture and the time of blood sample collection should be taken into account. In general, depending on the amino-acid mixture that was used, plasma TRP levels decrease more as the time between consumption and blood sample collection increases $^{32}$. This was also observed in studies using the ATD paradigm in PD patients ${ }^{19,42}$, so it might be assumed that plasma TRP levels continued to decline or remained stable during the further course of the test days. Moreover, it has been hypothesised that the threshold of decline should be defined in terms of the ratio between TRP levels and the large neutral amino acids, as these give a more accurate estimate of TRP availability to the brain than free TRP levels ${ }^{67}$. In the present study the reduction in TRP / $\Sigma$ LNAA ratio reached $60.9 \%$. It seems unlikely that the smaller lowering of TRP levels resulted in an effect on 5-HT function that was below the threshold to alter mood, as in the placebo condition which was marked by a profound increase in plasma TRP levels, no effect on mood could be detected compared to the ATD condition.

Another limitation of the ATD paradigm is that 5-HT depletion cannot be localised to particilar regions in the brain, which might impact the effect of ATD on the different outcome parameters. Lastly, it should be taken into account that participants were on their regular dopaminergic medication, which may have played a confounding role.

\section{CONCLUSION}

We have shown that 5-HT manipulation by ATD did not lead to dissociable effects on different measures of mood and emotional processing, motor symptoms and inhibitory control. These findings suggests that psychiatric side-effects following STN-DBS in PD patients cannot be directly linked to a reduction in 5-HT activity. 


\section{REFERENCES}

1. Lees AJ, Hardy J, Revesz T. Parkinson's disease. Lancet 2009;373(9680):2055-2066.

2. Rodriguez-Oroz MC, Jahanshahi M, Krack $P$, et al. Initial clinical manifestations of Parkinson's disease: features and pathophysiological mechanisms. Lancet Neurol 2009;8(12):1128-1139.

3. Deuschl G, Schade-Brittinger C, Krack P, et al. A randomized trial of deep-brain stimulation for Parkinson's disease. N Engl J Med 2006;355(9):896-908.

4. Okun MS. Deep-brain stimulation for Parkinson's disease. N Engl J Med 2012;367(16):15291538.

5. Krack P, Batir A, Van Blercom N, et al. Five-year follow-up of bilateral stimulation of the subthalamic nucleus in advanced Parkinson's disease. N Engl J Med 2003;349(20):1925-1934.

6. Weaver FM, Follett KA, Stern M, et al. Randomized trial of deep brain stimulation for Parkinson disease: thirty-six-month outcomes. Neurology 2012;79(1):55-65.

7. Thobois S, Ardouin C, Lhommee E, et al. Non-motor dopamine withdrawal syndrome after surgery for Parkinson's disease: predictors and underlying mesolimbic denervation. Brain 2010;133:1111-1127.

8. Temel Y, Kessels A, Tan S, Topdag A, Boon P, Visser-Vandewalle V. Behavioural changes after bilateral subthalamic stimulation in advanced Parkinson disease: a systematic review. Parkinsonism Relat Disord 2006;12(5):265-272.

9. Temel Y. Limbic effects of high-frequency stimulation of the subthalamic nucleus. Vitam Horm 2010;82:47-63.

10. Buhmann $\mathrm{C}$, Huckhagel $\mathrm{T}$, Engel $\mathrm{K}$, et al. Adverse events in deep brain stimulation: $\mathrm{A}$ retrospective long-term analysis of neurological, psychiatric and other occurrences. PLoS One 2017;12(7):e0178984.

11. Nazzaro JM, Pahwa R, Lyons KE. The impact of bilateral subthalamic stimulation on non-motor symptoms of Parkinson's disease. Parkinsonism Relat Disord 2011;17(8):606-609.

12. Floden D, Cooper SE, Griffith SD, Machado AG. Predicting quality of life outcomes after subthalamic nucleus deep brain stimulation. Neurology 2014;83(18):1627-1633.

13. Tan SK, Hartung H, Sharp T, Temel Y. Serotonin-dependent depression in Parkinson's disease: a role for the subthalamic nucleus? Neuropharmacology 2011;61(3):387-399.

14. Houeto JL, Mesnage V, Mallet L, et al. Behavioural disorders, Parkinson's disease and subthalamic stimulation. J Neurol Neurosurg Psychiatry 2002;72(6):701-707.

15. Faggiani E, Benazzouz A. Deep brain stimulation of the subthalamic nucleus in Parkinson's disease: From history to the interaction with the monoaminergic systems. Prog Neurobiol 2017;151:139156.

16. Tan SK, Hartung H, Visser-Vandewalle V, Steinbusch HW, Temel Y, Sharp T. A combined in vivo neurochemical and electrophysiological analysis of the effect of high-frequency stimulation of the subthalamic nucleus on 5-HT transmission. Exp Neurol 2012;233(1):145-153.

17. Temel Y, Boothman LJ, Blokland A, et al. Inhibition of 5-HT neuron activity and induction of depressive-like behavior by high-frequency stimulation of the subthalamic nucleus. Proc Natl Acad Sci U S A 2007;104(43):17087-17092.

18. Navailles S, Benazzouz A, Bioulac B, Gross C, De Deurwaerdere P. High-frequency stimulation of the subthalamic nucleus and L-3,4-dihydroxyphenylalanine inhibit in vivo serotonin release in the prefrontal cortex and hippocampus in a rat model of Parkinson's disease. J Neurosci 2010;30(6):23562364.

19. Scholtissen B, Verhey FR, Steinbusch HW, Leentjens AF. Serotonergic mechanisms in Parkinson's disease: opposing results from preclinical and clinical data. J Neural Transm (Vienna) 2006;113(1):59-73.

20. Marsh L. Depression and Parkinson's disease: current knowledge. Curr Neurol Neurosci Rep 2013;13(12):409.

21. Johansson B, Roos BE. 5-hydroxyindoleacetic and homovanillic acid levels in the cerebrospinal fluid of healthy volunteers and patients with Parkinson's syndrome. Life Sci 1967;6(13):1449-1454. 
22. Scatton B, Javoy-Agid F, Rouquier L, Dubois B, Agid Y. Reduction of cortical dopamine, noradrenaline, serotonin and their metabolites in Parkinson's disease. Brain Res 1983;275(2):321-328. 23. Birkmayer W, Birkmayer JD. Dopamine action and disorders of neurotransmitter balance. Gerontology 1987;33(3-4):168-171.

24. Kerenyi L, Ricaurte GA, Schretlen DJ, et al. Positron emission tomography of striatal serotonin transporters in Parkinson disease. Arch Neurol 2003;60(9):1223-1229.

25. Politis M, Wu K, Loane $\mathrm{C}$, et al. Staging of serotonergic dysfunction in Parkinson's disease: an in vivo 11C-DASB PET study. Neurobiol Dis 2010;40(1):216-221.

26. Reijnders JS, Ehrt U, Weber WE, Aarsland D, Leentjens AF. A systematic review of prevalence studies of depression in Parkinson's disease. Mov Disord 2008;23(2):183-189; quiz 313.

27. Temel Y, Tan S, Visser-Vandewalle V, Sharp T. Parkinson's disease, DBS and suicide: a role for serotonin? Brain 2009;132(Pt 10):e126; author reply e127.

28. Goetz CG, Tilley BC, Shaftman SR, et al. Movement Disorder Society-sponsored revision of the Unified Parkinson's Disease Rating Scale (MDS-UPDRS): scale presentation and clinimetric testing results. Mov Disord 2008;23(15):2129-2170.

29. Folstein MF, Folstein SE, McHugh PR. "Mini-mental state". A practical method for grading the cognitive state of patients for the clinician. J Psychiatr Res 1975;12(3):189-198.

30. Colasanti A, Esquivel G, den Boer E, et al. Effects of tryptophan depletion and tryptophan loading on the affective response to high-dose $\mathrm{CO} 2$ challenge in healthy volunteers. Psychopharmacology (Berl) 2011;215(4):739-748.

31. Sambeth A, Riedel WJ, Tillie DE, Blokland A, Postma A, Schmitt JA. Memory impairments in humans after acute tryptophan depletion using a novel gelatin-based protein drink. J Psychopharmacol 2009;23(1):56-64.

32. Evers EA, Tillie DE, van der Veen FM, et al. Effects of a novel method of acute tryptophan depletion on plasma tryptophan and cognitive performance in healthy volunteers. Psychopharmacology (Berl) 2005;178(1):92-99.

33. Knott V, Bisserbe JC, Shah D, et al. The moderating influence of nicotine and smoking on resting-state mood and EEG changes in remitted depressed patients during tryptophan depletion. Biol Psychol 2013;94(3):545-555.

34. Leentjens AF, Dujardin K, Pontone GM, Starkstein SE, Weintraub D, Martinez-Martin P. The Parkinson Anxiety Scale (PAS): development and validation of a new anxiety scale. Mov Disord 2014;29(8):1035-1043.

35. Hoehn MM, Yahr MD. Parkinsonism: onset, progression and mortality. Neurology 1967;17(5):427-442.

36. McNair DM, Lorr, M., \& Droppleman, L.F. . Revised manual for the Profile of Mood States. San Diego, CA: Educational and Industrial Testing Services, 1992.

37. Lang PJ, Bradley MM, Cuthbert BN. International affective picture system (IAPS): Affective ratings of pictures and instruction manual. Technical report A-8 2008.

38. Adam JJ, van Houdt H, Scholtissen B, Visser-Vandewalle V, Winogrodzka A, Duits A. Executive control in Parkinson's disease: effects of dopaminergic medication and deep brain stimulation on anticue keypress performance. Neurosci Lett 2011;500(2):113-117.

39. Adam JJ, Jennings S, Bovend'Eerdt TJ, Hurks PP, Van Gerven PW. Switch hands! Mapping temporal dynamics of proactive manual control with anticues. Acta Psychol (Amst) 2015;161:137-144.

40. Adam JJ, Ament B, Hurks P. Response preparation with anticues in children and adults. J Cogn Psychol 2011;23(2):264-271.

41. IBMCorp. IBM SPSS statistics for Windows. Version 24.0. Armonk, NY: IBM Corp, 2016.

42. Leentjens AF, Scholtissen B, Vreeling FW, Verhey FR. The serotonergic hypothesis for depression in Parkinson's disease: an experimental approach. Neuropsychopharmacology 2006;31(5):1009-1015.

43. Mace JL, Porter RJ, Dalrymple-Alford JC, Anderson TJ. The effects of acute tryptophan depletion on mood in patients with Parkinson's disease and the healthy elderly. J Psychopharmacol 2010;24(4):615-619. 
44. Yamamoto T, Uchiyama T, Higuchi $\mathrm{Y}$, et al. Subthalamic Nucleus Deep Brain Stimulation Modulate Catecholamine Levels with Significant Relations to Clinical Outcome after Surgery in Patients with Parkinson's Disease. PLoS One 2015;10(9):e0138462.

45. Young SN. Acute tryptophan depletion in humans: a review of theoretical, practical and ethical aspects. J Psychiatry Neurosci 2013;38(5):294-305.

46. Broen MP, Marsman VA, Kuijf ML, Van Oostenbrugge RJ, van Os J, Leentjens AF. Unraveling the Relationship between Motor Symptoms, Affective States and Contextual Factors in Parkinson's Disease: A Feasibility Study of the Experience Sampling Method. PLoS One 2016;11(3):e0151195.

47. Storch A, Schneider CB, Wolz M, et al. Nonmotor fluctuations in Parkinson disease: severity and correlation with motor complications. Neurology 2013;80(9):800-809.

48. Menza MA, Sage J, Marshall E, Cody R, Duvoisin R. Mood changes and "on-off" phenomena in Parkinson's disease. Mov Disord 1990;5(2):148-151.

49. Stansley BJ, Yamamoto BK. L-Dopa and Brain Serotonin System Dysfunction. Toxics 2015;3(1):75-88.

50. Moonen AJH, Weiss $\mathrm{PH}$, Wiesing $\mathrm{M}$, et al. An fMRI study into emotional processing in Parkinson's disease: Does increased medial prefrontal activation compensate for striatal dysfunction? PLoS One 2017;12(5):e0177085.

51. Phillips ML, Drevets WC, Rauch SL, Lane R. Neurobiology of emotion perception I: The neural basis of normal emotion perception. Biol Psychiatry 2003;54(5):504-514.

52. Ruhe HG, Mason NS, Schene AH. Mood is indirectly related to serotonin, norepinephrine and dopamine levels in humans: a meta-analysis of monoamine depletion studies. Mol Psychiatry 2007;12(4):331-359.

53. Moonen AJH, Wijers A, Dujardin K, Leentjens AFG. Neurobiological correlates of emotional processing in Parkinson's disease: A systematic review of experimental studies. J Psychosom Res 2017; 100:65-76.

54. Temel Y, Blokland A, Steinbusch HW, Visser-Vandewalle V. The functional role of the subthalamic nucleus in cognitive and limbic circuits. Prog Neurobiol 2005;76(6):393-413.

55. Brucke $\mathrm{C}$, Kupsch A, Schneider GH, et al. The subthalamic region is activated during valencerelated emotional processing in patients with Parkinson's disease. Eur J Neurosci 2007;26(3):767-774.

56. Dujardin K, Blairy S, Defebvre L, et al. Subthalamic nucleus stimulation induces deficits in decoding emotional facial expressions in Parkinson's disease. J Neurol Neurosurg Psychiatry 2004;75(2):202-208.

57. Peron J, Grandjean D, Le Jeune F, et al. Recognition of emotional prosody is altered after subthalamic nucleus deep brain stimulation in Parkinson's disease. Neuropsychologia 2010;48(4):10531062.

58. Peron J, Biseul I, Leray E, et al. Subthalamic nucleus stimulation affects fear and sadness recognition in Parkinson's disease. Neuropsychology 2010;24(1):1-8.

59. Benedetti F, Colloca L, Lanotte M, Bergamasco B, Torre E, Lopiano L. Autonomic and emotional responses to open and hidden stimulations of the human subthalamic region. Brain Res Bull 2004;63(3):203-211.

60. Kuhn AA, Hariz MI, Silberstein P, et al. Activation of the subthalamic region during emotional processing in Parkinson disease. Neurology 2005;65(5):707-713.

61. Peron J, Fruhholz S, Verin M, Grandjean D. Subthalamic nucleus: a key structure for emotional component synchronization in humans. Neurosci Biobehav Rev 2013;37(3):358-373.

62. Jahanshahi M, Rothwell JC. Inhibitory dysfunction contributes to some of the motor and nonmotor symptoms of movement disorders and psychiatric disorders. Philos Trans R Soc Lond B Biol Sci 2017;372(1718).

63. Jahanshahi M, Obeso I, Baunez $C$, Alegre M, Krack P. Parkinson's disease, the subthalamic nucleus, inhibition, and impulsivity. Mov Disord 2015;30(2):128-140.

64. Pattij T, Vanderschuren LJ. The neuropharmacology of impulsive behaviour. Trends Pharmacol Sci 2008;29(4):192-199.

65. Braver TS. The variable nature of cognitive control: a dual mechanisms framework. Trends Cogn Sci 2012;16(2):106-113. 
66. Van Gerven PWM, Hurks PPM, Adam JJ. Both facilitatory and inhibitory impairments underlie age-related differences of proactive cognitive control across the adult lifespan. Acta Psychol (Amst) 2017;179:78-88.

67. Van der Does AJ. The mood-lowering effect of tryptophan depletion: possible explanation for discrepant findings. Arch Gen Psychiatry 2001;58(2):200-202.

68. Hamilton M. A rating scale for depression. J Neurol Neurosurg Psychiatry 1960; 56-62.

69. Emmerling F, Duecker F, de Graaf T, Schuhmann T, Adam JJ, Sack AT. Foresight beats hindsight: The neural correlates underlying motor preparation in the pro-/anti-cue paradigm. Brain and Behavior 2017;7:e006663. 


\section{Supplementary materials}

Supplementary Table 1. Mean valence and arousal ratings of the emotional responsiveness task

$\begin{array}{cccc}\text { Stimuli } & \begin{array}{c}\text { Nr. of } \\ \text { stimuli }\end{array} & \text { Valence } & \text { Arousal }\end{array}$

Literature

Negative

Positive

Neutral

Version 1

$\begin{array}{ll}\text { Negative } & 15 \\ \text { Positive } & 15 \\ \text { Neutral } & 15 \\ \text { Total } & 45\end{array}$

Version 2

$\begin{array}{ll}\text { Negative } & 15 \\ \text { Positive } & 15 \\ \text { Neutral } & 15 \\ \text { Total } & 45\end{array}$

Version 3

$\begin{array}{ll}\text { Negative } & 15 \\ \text { Positive } & 15 \\ \text { Neutral } & 15 \\ \text { Total } & 45\end{array}$

\section{Version 4}

$\begin{array}{ll}\text { Negative } & 15 \\ \text { Positive } & 15 \\ \text { Neutral } & 15 \\ \text { Total } & 45\end{array}$

$\begin{array}{ll}2.3(0.6) & 6.4(0.5) \\ 7.3(0.5) & 6.0(0.6) \\ 5.1(0.3) & 3.0(0.4)\end{array}$

$$
\begin{array}{rr}
2.3(0.6)^{\mathrm{Nt}, P} & 6.4(0.5)^{\mathrm{Nt}} \\
7.4(0.4)^{\mathrm{Nt}, \mathrm{N}} & 5.8(0.7)^{\mathrm{Nt}} \\
5.3(0.7)^{\mathrm{N}, \mathrm{P}} & 3.4(1.2)^{\mathrm{N}, \mathrm{P}} \\
5.0(2.2) & 5.2(1.6)
\end{array}
$$

$\begin{array}{rr}2.2(0.5)^{\mathrm{Nt}, P} & 6.3(0.5)^{\mathrm{Nt}} \\ 7.4(0.6)^{\mathrm{Nt}, \mathrm{N}} & 6.0(0.7)^{\mathrm{Nt}} \\ 5.1(0.3)^{\mathrm{N}, \mathrm{P}} & 3.1(0.4)^{\mathrm{N}, P} \\ 4.9(2.2) & 5.1(1.6)\end{array}$

$$
\begin{array}{r}
2.3(0.6)^{\mathrm{Nt}, P} \\
7.5(0.4)^{\mathrm{Nt}, \mathrm{N}} \\
5.0(0.3)^{\mathrm{N}, P} \\
5.0(2.2)
\end{array}
$$$$
6.3(0.6)^{\mathrm{Nt}}
$$$$
5.9(0.7)^{\mathrm{Nt}}
$$$$
3.0(0.6)^{N, P}
$$$$
5.1(0.6)
$$

Data are presented as means (SD). ${ }^{\mathrm{N}}=$ Significant difference compared to Negative stimuli in same version at $p=0.00 .{ }^{N t}=$ Significant difference compared to Neutral stimuli in same version at $p=$ $0.00{ }^{P}=$ Significant difference compared to Positive stimuli in same version at $p=0.00$. Mean valence and arousal ratings are based on IAPS normative ratings ${ }^{37}$ 
Supplementary Table 2. Pictures from the International Affective Picture System (IAPS) included in the present study

\begin{tabular}{|c|c|c|c|c|c|}
\hline \multicolumn{2}{|c|}{ Neutral } & \multicolumn{2}{|c|}{ Positive } & \multicolumn{2}{|c|}{ Negative } \\
\hline 2002 & 7012 & 1650 & 5825 & 1120 & 6212 \\
\hline 2102 & 7014 & 1710 & 5833 & 1525 & 6231 \\
\hline 2191 & 7017 & 1811 & 7220 & 1930 & 6243 \\
\hline 2214 & 7020 & 2045 & 7230 & 2053 & 6260 \\
\hline 2215 & 7025 & 2058 & 7270 & 2095 & 6300 \\
\hline 2273 & 7026 & 2071 & 7330 & 2345.1 & 6312 \\
\hline 2357 & 7032 & 2075 & 7405 & 2683 & 6315 \\
\hline 2377 & 7034 & 2160 & 7470 & 2688 & 6540 \\
\hline 2382 & 7035 & 2216 & 7650 & 2691 & 6550 \\
\hline 2383 & 7036 & 2345 & 8030 & 2692 & 6560 \\
\hline 2393 & 7040 & 2347 & 8034 & 2703 & 6830 \\
\hline 2446 & 7041 & 4220 & 8080 & 2800 & 9075 \\
\hline 2745.1 & 7050 & 4520 & 8090 & 2981 & 9163 \\
\hline 2518 & 7052 & 4597 & 8158 & 3001 & 9183 \\
\hline 2570 & 7053 & 4598 & 8161 & 3030 & 9250 \\
\hline 2880 & 7056 & 4599 & 8163 & 3053 & 9252 \\
\hline 2890 & 7059 & 4607 & 8170 & 3060 & 9332 \\
\hline 5040 & 7060 & 4623 & 8179 & 3071 & 9410 \\
\hline 5130 & 7100 & 4660 & 8185 & 3102 & 9412 \\
\hline 5390 & 7175 & 4676 & 8186 & 3103 & 9413 \\
\hline 5471 & 7179 & 5001 & 8190 & 3110 & 9424 \\
\hline 5520 & 7190 & 5260 & 8210 & 3130 & 9433 \\
\hline 5731 & 7205 & 5450 & 8370 & 3170 & 9495 \\
\hline 5740 & 7217 & 5470 & 8380 & 3195 & 9570 \\
\hline 6150 & 7290 & 5480 & 8420 & 3266 & 9600 \\
\hline 7001 & 7491 & 5621 & 8470 & 3350 & 9635.1 \\
\hline 7002 & 7502 & 5623 & 8490 & 3400 & 9902 \\
\hline 7003 & 7547 & 5626 & 8499 & 3500 & 9908 \\
\hline 7004 & 7705 & 5629 & 8501 & 6021 & 9921 \\
\hline 7009 & 8312 & 5700 & 8502 & 6190 & 9940 \\
\hline
\end{tabular}


Supplementary Table 3. Affective scores on the POMS at baseline, 3.5 hours (with DBS ON) and 4.5 hours (with DBS OFF) after the intervention

\begin{tabular}{|c|c|c|c|c|c|c|}
\hline \multirow[b]{3}{*}{ Time (h) } & \multicolumn{3}{|c|}{ Placebo } & \multicolumn{3}{|c|}{ ATD } \\
\hline & Baseline & DBS ON & DBS OFF & Baseline & DBS ON & DBS OFF \\
\hline & 0 & 3.5 & 4.5 & 0 & 3.5 & 4.5 \\
\hline$\overline{\text { Total }}$ & $7.43(5.6)$ & $6.86(4.6)$ & 21.86 (12.9) & $4.86(3.6)$ & $11.14(11.2)$ & $23.14(13.3)$ \\
\hline Total corr. & $4.14(4.4)$ & $2.43(2.2)$ & $11.86(10.8)$ & $2.29(2.3)$ & $5.57(10.8)$ & $13.00(11.9)$ \\
\hline Sadness & $2.29(3.9)$ & $0.86(0.9)$ & $6.29(5.1)$ & $1.14(1.6)$ & $2.14(4.4)$ & $6.43(5.6)$ \\
\hline Anger & $0.71(1.2)$ & $0.57(0.8)$ & $2.43(4.4)$ & $0.00(0.0)$ & $1.86(4.9)$ & $1.71(3.3)$ \\
\hline Fatigue & $3.00(1.9)$ & $4.43(2.9)$ & $9.57(5.8)$ & $2.43(1.5)$ & $5.57(1.8)$ & $10.14(3.8)$ \\
\hline Vigour & $12.86(2.0)$ & $9.29(4.3)$ & $7.00(4.1)$ & $10.86(5.0)$ & $9.71(4.0)$ & $6.43(3.7)$ \\
\hline Tension & $1.43(1.3)$ & $1.29(1.3)$ & $4.71(2.7)$ & $1.29(1.4)$ & $1.57(1.7)$ & 4.57 (3.8) \\
\hline
\end{tabular}





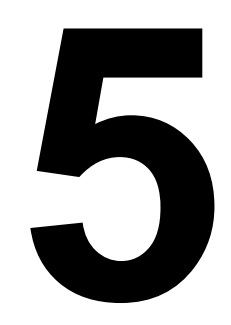

\section{The association between surgical characteristics and cognitive decline following deep brain stimulation of the subthalamic nucleus in Parkinson's disease}

Anne Mulders, Yasin Temel, Mehmet Tönge, Frédéric Schaper, Vivianne van Kranen-Mastenbroek, Linda Ackermans, Pieter Kubben, Marcus Janssen, Annelien Duits (submitted) 


\section{ABSTRACT}

Objective: despite optimal improvement in motor functioning, both short- and longterm studies have reported small but consistent changes in cognitive functioning following STN-DBS in Parkinson's disease (PD). The aim of the present study was to explore whether surgical characteristics were associated with cognitive decline one year following STN-DBS.

Methods: we retrospectively analysed 49 PD patients who underwent bilateral STNDBS. Cognitive change scores were related to the number of microelectrode recording (MER) trajectories, the STN length as measured by MER, and cortical entry points. Regression analyses were corrected for age at surgery, disease duration, education and preoperative levodopa responsiveness. Patients were then divided into a cognitive and non-cognitive decline group for each neuropsychological test and compared regarding demographic and surgical characteristics.

Results: one year postoperatively, significant declines were found in verbal fluency, Stroop Colour-Word test and Trail Making Test B (TMT-B). Only changes in TMT-B were associated with the coronal entry point in the right hemisphere. The number of MER trajectories and STN length were not associated with cognitive change scores. When comparing the cognitive decline and non-cognitive decline groups, no significant differences were found in surgical characteristics.

Conclusions: the electrode passage through the right prefrontal lobe may contribute to subtle changes in executive function. However, only few patients showed clinically relevant cognitive decline. The use of multiple MER trajectories and a longer STN length were not associated with cognitive decline one year following surgery. From a cognitive point of view, DBS may be considered a relatively safe procedure. 


\section{INTRODUCTION}

Deep brain stimulation (DBS) of the subthalamic nucleus (STN) is an effective and widely accepted treatment for Parkinson's disease (PD), and associated with improved motor functioning and quality of life ${ }^{1,2}$. However, both short-term and long-term followup studies have reported small but reliable changes in cognitive functioning, especially in the executive function domain ${ }^{3-7}$. Several patient inherent characteristics have been associated with cognitive decline following STN-DBS, such as higher age, levodopa dosage, impaired attention, and axial symptoms ${ }^{8,9}$. Moreover, stimulation and surgical factors such as the location of the active electrode, the volume of tissue activated, and stimulation settings may partially explain changes in cognitive functioning ${ }^{10-13}$. It has also been suggested that cognitive decline following STN-DBS reflects a microlesion effect due to the trajectories used during surgery, such as trajectories intersecting the caudate nuclei ${ }^{4}, 12,14-18$.

Accurate placement of the electrode within the dorsolateral sensorimotor part of the STN is considered necessary for satisfactory outcome. To date, microelectrode recording (MER) is typically used to accurately detect the STN and to optimise lead implantation. A recent study showed that multiple simultaneously inserted microelectrodes may provide better guidance than single sequential microelectrodes ${ }^{19}$. Moreover, prefrontal entry points are ideally chosen in order to maximise the length of the DBS electrode within the STN, thereby offering more contact combinations for postoperative DBS programming 20,21 . However, it has been hypothesised that a greater number of MER trajectories could result in a greater degree of local injury, presumably through damage to functional areas along the trajectory or at the target site important for cognitive functioning, thereby contributing to cognitive decline following surgery ${ }^{14,16,22}$. Moreover, maximising the STN's length may increase the chance of affecting the STN's associative area, hence leading to cognitive decline ${ }^{16}$.

The goal of the present study was to investigate whether characteristics of the surgical trajectory, including the number of MER trajectories, the STN length as measured by MER, and the angles (cortical entry points) of the lead trajectories, are associated with cognitive decline one year following STN-DBS in PD patients. Furthermore, we tested associations between these characteristics and motor improvement one year following 
surgery. The results may help in optimising the surgical procedure by balancing the benefits and the disadvantages to attempt for maximal STN length.

\section{METHODS}

\section{Design and participants}

This study was conducted at Maastricht University Medical Centre and was exempted from ethical approval by the local ethical committee (METC azm/UM). Informed consent was not obtained from the patients because of the retrospective nature of the study. All patients $(\mathrm{N}=81)$ who underwent bilateral STN-DBS for Parkinson's disease between 2005 and March 2017 were retrospectively identified (see Supplementary table 1). Inclusion criteria for our study were: (1) pre- and postoperative neuroimaging sufficient for determining the surgical trajectory, (2) MER recordings, (3) available preand one year post-neuropsychological assessment, and (4) no complications or reimplantation of electrodes. A total of 49 patients were found eligible for the study (see Supplementary table 2 for reasons for exclusion), of which 20 patients were also included in an earlier study ${ }^{12}$.

\section{Surgical details}

The surgical procedure has been described in detail previously ${ }^{23}$ and included direct targeting of the STN and MER guidance during surgery. Trajectory planning was performed on gadolinium enhanced T1 images using dedicated surgical planning software (Framelink, Medtronic, Minneapolis, USA). The length of the intercommissural line (AC-PC line), coordinates with respect to mid-commissural point (MCP) and both sagittal and coronal angles of the trajectory were assessed by a neurosurgeon. When applicable, up to five MER trajectories per side were used. In case of the presence of vessels, the respective trajectories were discarded. MER were initiated $10 \mathrm{~mm}$ above the target point and continued with $1.0 \mathrm{~mm}$ steps. From $5 \mathrm{~mm}$ above target, steps of 0.25-0.50 mm were used for MER until STN activity disappeared and/or until the typical substantia nigra activity appeared, as assessed by a clinical neurophysiologist. Trajectories with the longest STN activity were chosen for test stimulation. The trajectory with the least side-effects and highest effect on the key symptom with largest stimulation window (amplitude threshold of therapeutic and sideeffects) was chosen for the placement of the final electrode. The length of this final trajectory as measured by MER was recorded in mm (STN length). 


\section{MRI analysis}

All patients had a postoperative CT or MR scan for evaluation of postoperative complications and electrode localisation. Pre-and postoperative images were imported into the Framelink 5.1 ${ }^{\mathrm{TM}}$ (Medronic BV, Minneapolis HQ, USA) software. After orientation of the data set had been confirmed, the preoperative MRI was automatically fused with the postoperative MRI or CT for analysis of the postoperative surgical trajectory. Subsequently, a neurosurgeon created linear vectors between the observed lead tip and cortical entry point in multiplanar and probe's eye view of all 98 DBS electrode trajectories (both hemispheres in each patient) and determined the coronal and sagittal angles of the entry points. For the coronal entry point (named mid-sagittal plane in the software), angles closer to $0^{\circ}$ were located to the midpoint of the AC-PC line, while angles close to $90^{\circ}$ were located laterally. For the sagittal entry point (named axial plane in the software) angles closer to $0^{\circ}$ were closer to the midpoint of the ACPC line, and angles closer to $90^{\circ}$ were located dorsally.

\section{Neuropsychological and motor assessment}

Neuropsychological assessment was performed one month before surgery and one year after surgery. Preoperatively, patients were tested with medication 'ON', and postoperatively with medication and stimulation 'ON'. The tests were part of the standard protocol for PD patients receiving STN-DBS. If available, alternating forms were used at follow-up time points to minimise practice effects. For the current study, we selected the Auditory Verbal Learning Test (AVLT) immediate and delayed recall ${ }^{24}$. The category (animals and professions) and letter fluency test were used as measures of semantic and phonemic verbal fluency, respectively ${ }^{25}$. The Stroop Colour-Word Test was used to measure mental speed and response inhibition ${ }^{26}$. The Trail Making Test (TMT) (part A and B) were used to assess mental speed and cognitive flexibility, respectively ${ }^{27}$.

Motor state was assessed by the Unified Parkinson's Disease Rating Scale (UPDRS) part III. Preoperatively, patients were tested in 'ON' and 'OFF' medication state, while postoperatively assessments were performed with stimulation and medication 'on'. Percentage improvement in motor disability was determined in respect of the preoperative OFF-medication condition. 


\section{Statistics}

Statistical analyses were performed using IBM SPSS Statistics 25.028. Possible differences in STN length, cortical entry points and number of MER trajectories between the right and left hemisphere were analysed using paired sample t-test. Changes in clinical and neuropsychological variables following DBS were analysed using parametric t-test statistics and non-parametric Wilcoxon-Rank order tests when appropriate (lack of normal distribution of data). A p-value $<0.05$ was considered to be statistically significant. No correction for multiple comparisons was applied in order to reduce the risk of type II error given the importance of detecting any adverse effects of surgery. Statistically significant mean differences underwent Cohen's d calculation for effect size.

Multiple regression analyses were performed to analyse the associations between surgical characteristics and both cognitive decline and motor improvement one year following surgery. The dependent variables were raw change scores of the neuropsychological tests that showed a statistically significant decline following surgery and the percentage of UPDRS III improvement, respectively. The predictor variables were the surgical characteristics, each tested in a separate model for both left and right hemisphere, and adjusted for age, education, disease duration and Levodopa equivalent daily dose (LEDD) at baseline. For the regression analyses, a Bonferroni adjusted $p$-value $<0.001$ was considered statistically significant.

Lastly, for each neuropsychological test that showed statistically significant change following surgery, patients were categorised into two distinct groups based on the presence or absence of cognitive decline: the cognitive decline group and the noncognitive decline group (or stable performers). For this purpose, standardised Z-score were calculated at baseline and follow-up based on the mean and standard deviation of the total sample at baseline. Cognitive decline was defined as a raw score change (decline for verbal fluency and AVLT, and increase for TMT, Stoop Colour-Word test) $>1$ Z-score from baseline. Groups (cognitive decline yes / no) were then compared using non-parametric Mann-Whitney tests for demographic and surgical characteristics. For the comparison analyses, a Bonferroni adjusted $p$-value $<0.002$ was considered statistically significant. 


\section{RESULTS}

\section{Patient and surgical characteristics}

The study population consisted of 31 males and 18 females. The average age at of PD onset was 49.0 years $(S D=9.6)$ and the average age at DBS surgery was 60.1 years $(S D=8.5)$. The average disease duration was 133.7 months $(S D=31.7)$. Mean UPDRS part III score at baseline was 37.7 (12.0) and the average LEDD was 1346.1 $\mathrm{mg}(\mathrm{SD}=329.9)$.

Surgical characteristics are provided in Table 1. In 32 patients the right hemisphere was operated first. The average STN length was $5.2 \mathrm{~mm}(\mathrm{SD}=1.5)$, the mean sagittal angle was 56.6 degrees (SD = 8.9) and the mean coronal angle was 23.3 degrees (SD = 5.1). There were no statistically significant differences between the right and left hemispheres regarding STN length, sagittal and coronal angle (coronal entry points) and number of MER trajectories.

Table 1. Surgical characteristics

\begin{tabular}{|c|c|c|c|c|}
\hline & $\begin{array}{l}\text { Right hemisphere } \\
(\mathrm{N})\end{array}$ & $\begin{array}{l}\text { Left hemisphere } \\
(\mathrm{N})\end{array}$ & $\begin{array}{l}\text { Total } \\
(\mathrm{N})\end{array}$ & \\
\hline \multicolumn{5}{|l|}{ MER trajectories* } \\
\hline-1 & 0 & 0 & 0 & \\
\hline-2 & 3 & 1 & 4 & \\
\hline-3 & 14 & 17 & 31 & \\
\hline-4 & 21 & 24 & 45 & \\
\hline-5 & 10 & 6 & 16 & \\
\hline \multicolumn{5}{|l|}{ Chosen trajectory** } \\
\hline - anterior & 4 & 4 & 8 & \\
\hline - medial & 6 & 3 & 9 & \\
\hline - central & 29 & 34 & 63 & \\
\hline - lateral & 5 & 5 & 10 & \\
\hline - posterior & 3 & 2 & 5 & \\
\hline \multirow[t]{2}{*}{ First side operation } & 32 & 17 & & \\
\hline & Right & Left & Total & $P$ value \\
\hline STN length & $5.1(1.5)$ & $5.3(1.4)$ & $5.2(1.5)$ & 0.25 \\
\hline Sagittal angle & $56.3(8.9)$ & $56.9(9.1)$ & $56.6(8.9)$ & 0.45 \\
\hline Coronal angle & $23.3(5.1)$ & $23.7(5.0)$ & $23.5(5.0)$ & 0.67 \\
\hline $\begin{array}{l}\mathrm{Nr} \text { of MER (median, } \\
\text { range) }{ }^{*}\end{array}$ & $4(2-5)$ & $4(2-5)$ & $4(2-5)$ & 0.52 \\
\hline \multicolumn{5}{|c|}{$\begin{array}{l}\text { Nr of MER: number of electrodes used for microelectrode recording registration, STN = } \\
\text { nucleus } \\
\text { Results are presented as means and standard deviations, unless otherwise specified } \\
\text { * Data missing for } 1 \text { patient } \\
\text { ** Data missing for } 1 \text { patient bilaterally and for } 1 \text { patient unilaterally }\end{array}$} \\
\hline
\end{tabular}




\section{Neuropsychological and motor outcome following STN-DBS}

One year following surgery there were statistically significant changes in Stroop Colour-Word test performance (medium effect size), in TMT-B performance (small effect size) and in both category and letter fluency performance (large effect size) (Table 2). All changes reflect a decline in cognitive functioning. LEDD decreased by $54 \%$ and UPDRS part III improved by $50.1 \%$ (table 3), all on a group level.

Table 2. Neuropsychological test scores at baseline, 1 year following DBS surgery and test change scores (T1 minus T0). Means, standard deviations and Wilcoxen signed rank analyses or $\mathrm{T}$ test are shown

\begin{tabular}{l|c|c|c|c|c|c}
\hline & N & Baseline & $\begin{array}{c}\text { 1 year follow } \\
\text { up }\end{array}$ & $\begin{array}{c}\text { Change } \\
\text { score }\end{array}$ & P-value & $\begin{array}{c}\text { Cohen's } \\
\mathrm{d}\end{array}$ \\
\hline Stroop I & 49 & $51.0(12.0)$ & $55.6(15.8)$ & $4.6(8.5)$ & 0.000 & 0.54 \\
Stroop II & 49 & $66.9(15.1)$ & $76.7(25.1)$ & $9.8(16.4)$ & 0.000 & 0.60 \\
Stroop III & 48 & $113.4(38.8)$ & $143.4(82.5)$ & $29.8(64.1)$ & 0.000 & 0.46 \\
Stroop Int & 48 & $50.1(32.3$ & $74.1(69.9)$ & $24.1(59.1)$ & 0.000 & 0.41 \\
TMT A & 46 & $39.8(15.0)$ & $42.4(18.6)$ & $1.8(12.4)$ & 0.46 & \\
TMT B & 45 & $104.0(69.2)$ & $116.8(76.7)$ & $12.8(41.9)$ & 0.047 & 0.31 \\
AVLT total & 48 & $43.5(10.9)$ & $43.9(10.7)$ & $0.4(2.9)$ & 0.69 & \\
AVLT recall & 48 & $8.7(3.2)$ & $8.4(3.2)$ & $-.33(2.9)$ & 0.43 & \\
Fluency category & 49 & $41.3(9.8)$ & $33.9(11.5)$ & $-7.4(8.4)$ & 0.000 & 0.89 \\
- animals & & $23.8(5.2)$ & $19.5(6.5)$ & $-4.3(5.0)$ & 0.000 & 0.86 \\
- occupations & & $18.0(5.5)$ & $14.8(5.6)$ & $-3.2(4.2)$ & 0.000 & 0.76 \\
Fluency letters & 48 & $34.3(11.4)$ & $29.8(12.3)$ & $-4.6(8.2)$ & 0.000 & 0.56 \\
\hline
\end{tabular}

Stroop $=$ Stroop Colour-Word test, Int = interference score, TMT = Trail Making Test, AVLT = Auditory Verbal Learning test

Table 3. UPDRS part III and LEDD scores at baseline and 1 year following DBS surgery and change scores. Means, standard deviations and $\mathrm{T}$ test analyses are shown

\begin{tabular}{lccccc}
\hline & $\mathrm{N}$ & Baseline & 1 year follow up & Change score & P-value \\
\hline LEDD (mg/day) & 47 & $1346.1(629.9)$ & $617.9(476.5)$ & $-728.2(466.9)$ & $<0.001$ \\
UPDRS III OFF & 40 & $37.7(12.5)$ & $18.8(9.8)$ & $-18.9(14.0)$ & $<0.001$ \\
UPDRS III ON & 41 & $19.3(11.5)$ & $18.4(9.5)$ & $-.94(12.3)$ & 0.63
\end{tabular}

Patients were scored post-operatively with stimulation and medication 'on'

LEDD = levodopa equivalent daily dose, UPDRS = Unified Parkinson Disease Rating Scale, OFF = compared to baseline 'off' medication, $\mathrm{ON}=$ compared to baseline 'on' medication 
Table 4. Comparisons of demographic and surgical factors between the cognitive decline vs the non-cognitive decline group in Stroop ColourWord test III performance, TMT-B performance and verbal fluency. Means, standard deviations and Mann-Whitney test analyses are shown

\begin{tabular}{|c|c|c|c|c|c|c|c|c|c|c|c|c|}
\hline & \multicolumn{3}{|c|}{ Stroop III } & \multicolumn{3}{|c|}{ TMT-B } & \multicolumn{3}{|c|}{ Fluency category } & \multicolumn{3}{|c|}{ Fluency letters } \\
\hline $\mathrm{N}$ & $\begin{array}{l}\text { Yes } \\
5\end{array}$ & $\begin{array}{l}\text { No } \\
44\end{array}$ & $P$ & $\begin{array}{c}\text { Yes } \\
5\end{array}$ & $\begin{array}{l}\text { No } \\
40\end{array}$ & $P$ & $\begin{array}{c}\text { Yes } \\
8\end{array}$ & $\begin{array}{l}\text { No } \\
41\end{array}$ & $P$ & $\begin{array}{c}\text { Yes } \\
9\end{array}$ & $\begin{array}{l}\text { No } \\
39\end{array}$ & $p$ \\
\hline Age at surgery & $71.0(2.4)$ & $59.1(8.0)$ & $\begin{array}{c}0.00 \\
1\end{array}$ & $69.0(8.4)$ & $58.6(6.0)$ & $\begin{array}{c}0.01 \\
1\end{array}$ & $65.0(8.8)$ & $59.1(8.2)$ & 0.09 & $\begin{array}{c}60.8 \\
(10.8)\end{array}$ & $59.7(8.1)$ & 0.68 \\
\hline Disease duration & $\begin{array}{l}100.8 \\
(79.9)\end{array}$ & $\begin{array}{l}138.7 \\
(59.7)\end{array}$ & 0.10 & $\begin{array}{c}117.6 \\
(116.8)\end{array}$ & $\begin{array}{l}132.0 \\
(55.9)\end{array}$ & 0.13 & $\begin{array}{l}123.0 \\
(40.4)\end{array}$ & $\begin{array}{l}135.8 \\
(65.5)\end{array}$ & 0.66 & $\begin{array}{l}154.7 \\
(85.8)\end{array}$ & $\begin{array}{l}127.4 \\
(55.4)\end{array}$ & 0.50 \\
\hline LEDD (mg/day) & $\begin{array}{c}711.6 \\
(489.6)\end{array}$ & $\begin{array}{l}1432.5 \\
(609.0)\end{array}$ & $\begin{array}{c}0.01 \\
3\end{array}$ & $\begin{array}{c}687.0 \\
(450.8)\end{array}$ & $\begin{array}{l}1416.4 \\
(621.0)\end{array}$ & $\begin{array}{c}0.01 \\
0\end{array}$ & $\begin{array}{l}1338.5 \\
(822.3)\end{array}$ & $\begin{array}{l}1347.6 \\
(596.4)\end{array}$ & 0.79 & $\begin{array}{l}1297.8 \\
(459.8)\end{array}$ & $\begin{array}{l}1352.7 \\
(677.0)\end{array}$ & 0.85 \\
\hline $\begin{array}{l}\text { Education (range) } \\
\text { Right }\end{array}$ & $4.6(3-6)$ & $5.2(2-7)$ & 0.10 & $4.6(3-6)$ & $5.2(2-7)$ & 0.37 & $5.1(3-6)$ & $5.1(2-7)$ & 0.97 & $5.0(4-7)$ & $5.2(2-7)$ & 0.50 \\
\hline $\begin{array}{l}\text { MER trajectories } \\
\text { (range) }\end{array}$ & $3.8(3-5)$ & $3.8(2-5)$ & 0.41 & $3.3(3-4)$ & $3.8(2-5)$ & 0.19 & $3.8(2-5)$ & $3.8(2-5)$ & 0.97 & $3.8(2-5)$ & $3.8(2-5)$ & 0.99 \\
\hline STN length & $4.1(2.3)$ & $5.1(1.3)$ & 0.41 & $4.0(2.2)$ & $5.2(1.4)$ & 0.33 & $\begin{array}{l}(5.3) \\
(1.0)\end{array}$ & $5.0(1.5)$ & 0.99 & $4.7(1.4)$ & $5.2(1.4)$ & 0.45 \\
\hline Sagittal angle & $58.8(9.7)$ & $56.0(9.5)$ & 0.53 & $59.2(9.3)$ & $56.8(8.7)$ & 0.54 & $\begin{array}{c}57.6 \\
(11.8)\end{array}$ & $56.0(8.2)$ & 0.64 & $52.8(4.8)$ & $56.9(9.4)$ & 0.23 \\
\hline Coronal angle & $28.5(4.8)$ & $22.9(4.9)$ & $\begin{array}{c}0.02 \\
5\end{array}$ & $28.8(4.3)$ & $22.7(4.6)$ & $\begin{array}{c}0.00 \\
7\end{array}$ & $23.6(6.3)$ & $23.3(5.0)$ & 0.84 & $22.2(7.2)$ & $23.8(4.6)$ & 0.26 \\
\hline $\begin{array}{l}\text { Left } \\
\text { MER trajectories } \\
\text { (range) }\end{array}$ & $3.5(3-4)$ & $3.8(2-5)$ & 0.13 & $3.5(3-4)$ & $3.8(2-5)$ & 0.57 & $4.0(3-5)$ & $3.7(2-5)$ & 0.25 & $3.9(3-5)$ & $3.7(2-5)$ & 0.60 \\
\hline STN length & $4.1(2.1)$ & $5.4(1.3)$ & 0.13 & $4.1(2.2)$ & $5.5(1.2)$ & 0.11 & $5.5(1.7)$ & $5.2(1.4)$ & 0.52 & $4.8(1.6)$ & $5.4(1.4)$ & 0.32 \\
\hline Sagittal angle & $59.7(6.5)$ & $56.6(9.5)$ & 0.36 & $59.9(6.5)$ & $56.8(9.8)$ & 0.34 & $\begin{array}{c}57.3 \\
(13.4)\end{array}$ & $56.8(8.3)$ & 0.62 & $57.8(4.7)$ & $\begin{array}{c}56.7 \\
(10.0)\end{array}$ & 0.48 \\
\hline Coronal angle & $23.0(5.0)$ & $23.8(5.1)$ & 0.77 & $24.3(5.2)$ & $23.8(4.7)$ & 0.88 & $26.1(6.9)$ & $23.2(4.5)$ & 0.23 & $23.7(3.6)$ & $23.7(5.3)$ & 0.76 \\
\hline
\end{tabular}

Stroop = Stroop Colour-Word test part III, TMT = Trail Making Test, LEDD = Levodopa equivalent daily dose, MER trajectories = number of electrodes used for microelectrode recording registration. 


\section{Associations between surgical characteristics and both cognitive decline and motor improvement}

Multiple regression analyses were run to test the associations between surgical characteristics and cognitive decline (each tested in a separate model), age, education, disease-duration and LEDD at baseline. Changes in TMT-B performance were statistically significantly associated with right coronal angle: $F(5,37)=5.975, p$ $<0.00125, \mathrm{R}^{2}=0.447$, and only right coronal angle added statistically significantly to the model: $p<0.00125$. The surgical characteristics were not associated with changes in any of the other neuropsychological tests, nor with improvement in UPDRS III scores.

\section{Cognitive decline}

Patients were categorised into a cognitive decline or non-cognitive decline group for each neuropsychological test separately (see Table 4). Patients with cognitive decline in Stroop Colour-Word (card III) performance had a higher age at surgery $(p=0.001)$, compared to patients without cognitive decline. For the other neuropsychological tests, there were no statistically significant differences between the cognitive decline and non-cognitive decline groups.

\section{DISCUSSION}

In this retrospective study we investigated whether characteristics of the surgical trajectory, including the number of MER trajectories, the STN length as measured by MER and angles (cortical entry points) of the surgical trajectory, were associated with cognitive decline and motor improvement one year following STN-DBS in PD patients. One year postoperatively, we found significant declines in verbal fluency, Stroop Colour-Word test and TMT-B. Changes in TMT-B were associated with the coronal entry point in the right hemisphere, independently of age, education, disease duration, and LEDD at baseline. When comparing patients with and without clinically relevant cognitive decline in TMT-B performance, no differences in any of the surgical characteristics could be found. Motor improvement was not associated with characteristics of the surgical trajectories.

The declines as found in tests that measure executive function are consistent with other reports on cognitive (side-)effects of STN-DBS ${ }^{29}$. Similar to previous studies ${ }^{10,14}$ 
the number of MER trajectories used for accurate lead placement was not associated with cognitive decline one year after surgery. Moreover, no associations were found between STN length as measured by MER and cognitive or motor outcome, respectively, which is in line with previous studies ${ }^{16,30,31}$. Although these results cannot exclude a transient microlesion effect as proposed by others ${ }^{22}$, these findings suggest that there is no increased risk for cognitive decline at one year following surgery when increasing the number of MER trajectories or with maximising the STN length.

Changes in TMT-B performance, though showing a small effect size, were associated with the cortical entry point in the right hemisphere only, whereas we found no association between the left hemisphere and changes in TMT-B performance. Interestingly, several other studies observed hemispheric differences when studying the relationship between the lead trajectories and cognitive decline ${ }^{10,11,16}$. La Goff and colleagues (2015), for example, found that patients with a decline in semantic verbal fluency had a left trajectory with a more anterior cortical entry point ${ }^{16}$, while the present study did not find any relationship between cortical entry points and decline in verbal fluency performance. Changes in both semantic and phonemic verbal fluency following surgery, with moderate to large effect sizes, are one of the most common and robust findings in the literature ${ }^{4}, 22,32-34$, and there have been inconsistent findings regarding the associations between changes in verbal fluency performance following DBS and the electrode trajectories. Tröster and colleagues (2017) found differences between the effect of surgery and stimulation on semantic and phonemic fluency, respectively, suggesting that only semantic fluency is affected by the lead trajectory ${ }^{18}$. On the contrary, Okun and colleagues (2012) proposed that phonemic verbal fluency was the result of the lead placement, because a similar degree of decline was observed in both on-stimulation and off-stimulation states ${ }^{35}$. Another study demonstrated microstructural injuries along the electrode trajectories in white matter bundles that are implicated in verbal fluency following STN-DBS ${ }^{15}$. In general, executive function tests such as verbal fluency and TMT-B are difficult to interpret as they rely on a variety of cognitive processes. Semantic and phonemic verbal fluency depend on shared and distinct distributed brain regions, including the left inferior frontal gyrus, anterior cingulate gyrus, left frontal regions and temporal networks ${ }^{15}$. Performance on TMT-B involves divided and visual attention, cognitive flexibility, speed of processing, setshifting and working memory, which are most likely mediated by widespread activation 
of the bilateral PFC, as well as dorsomedial and dorsolateral regions ${ }^{36-39}$. As such, the findings in the present study could be regarded as non-specific to these numerous aspects of executive functioning. Importantly, the association between the right coronal entry point and TMT-B is based on changes in neuropsychological test performance and does not necessarily translate into impairment in daily activities. Only five out of 46 patients showed clinically relevant decline in TMT-B performance, and these five patients did not differ in terms of surgical characteristics compared to the non-decline group.

The literature so far shows inconsistent results with respect to the surgical impact on cognition. This inconsistency is due to many methodological differences, including differences in follow-up period, definitions of decline and assessment, but overall the sample sizes are relatively small, including the present study. Besides these methodological issues, changes in cognitive functioning rely upon a complex interplay of numerous factors, including age, levodopa-response, disease-duration and diseaseprogression, stimulation factors, preoperative motor symptoms, preoperative cognitive functioning and morphometric measures of brain atrophy, 40 which can hardly be examined in one study. Further effort should be put in data sharing and multicenter studies to increase sample sizes and thereby overall power. Additionally, study designs with neuropsychological assessment in medication-ON, and both stimulation ON and OFF condition, may exclude (or demonstrate) a stimulation effect and, as such, relieve some pressure of the power.

\section{CONCLUSION}

The electrode passage through the right prefrontal lobe may contribute to subtle changes in executive function. However, only few patients showed clinically relevant cognitive decline and as such the impact is low. More importantly, while the use of multiple MER trajectories and a longer STN length were not associated with cognitive decline one year following surgery, we were also not able to observe a gain in motor improvement when using multiple MER trajectories and a longer STN length. To conclude, from a cognitive point of view, DBS may be considered a relatively safe procedure. Surgical teams that are ambitious of maximising the length of the DBS electrode within the STN do not have to be restrained by a risk of cognitive side-effects, but still have to prove its clinical benefits. 


\section{REFERENCES}

1. Deuschl G, Schade-Brittinger $\mathrm{C}$, Krack $\mathrm{P}$, et al. A randomized trial of deep-brain stimulation for Parkinson's disease. N Engl J Med 2006;355(9):896-908.

2. Okun MS. Deep-Brain Stimulation for Parkinson's Disease. New Engl J Med 2012;367(16):1529-1538.

3. Smeding HM, Speelman JD, Koning-Haanstra M, et al. Neuropsychological effects of bilateral STN stimulation in Parkinson disease: a controlled study. Neurology 2006;66(12):1830-1836.

4. Witt K, Daniels C, Reiff J, et al. Neuropsychological and psychiatric changes after deep brain stimulation for Parkinson's disease: a randomised, multicentre study. Lancet Neurol 2008;7(7):605-614.

5. Saint-Cyr JA, Trepanier LL, Kumar R, Lozano AM, Lang AE. Neuropsychological consequences of chronic bilateral stimulation of the subthalamic nucleus in Parkinson's disease. Brain 2000;123 ( Pt 10):2091-2108.

6. Parsons TD, Rogers SA, Braaten AJ, Woods SP, Troster Al. Cognitive sequelae of subthalamic nucleus deep brain stimulation in Parkinson's disease: a meta-analysis. Lancet Neurol 2006;5(7):578588.

7. Janssen ML, Duits AA, Turaihi AH, et al. Subthalamic nucleus high-frequency stimulation for advanced Parkinson's disease: motor and neuropsychological outcome after 10 years. Stereotact Funct Neurosurg 2014;92(6):381-387.

8. Smeding HM, Speelman JD, Huizenga HM, Schuurman PR, Schmand B. Predictors of cognitive and psychosocial outcome after STN DBS in Parkinson's Disease. J Neurol Neurosurg Psychiatry 2011;82(7):754-760.

9. Witt K, Daniels C, Volkmann J. Factors associated with neuropsychiatric side effects after STNDBS in Parkinson's disease. Parkinsonism Relat Disord 2012;18 Suppl 1:S168-170.

10. Witt K, Granert O, Daniels C, et al. Relation of lead trajectory and electrode position to neuropsychological outcomes of subthalamic neurostimulation in Parkinson's disease: results from a randomized trial. Brain 2013;136:2109-2119.

11. York MK, Wilde EA, Simpson R, Jankovic J. Relationship between neuropsychological outcome and DBS surgical trajectory and electrode location. J Neurol Sci 2009;287(1-2):159-171.

12. Isler C, Albi A, Schaper FL, Temel Y, Duits A. Neuropsychological Outcome in Subthalamic Nucleus Stimulation Surgeries with Electrodes Passing through the Caudate Nucleus. Stereotact Funct Neurosurg 2016;94(6):413-420.

13. Eisenstein SA, Koller JM, Black KD, et al. Functional anatomy of subthalamic nucleus stimulation in Parkinson disease. Ann Neurol 2014;76(2):279-295.

14. Smith KM, O'Connor M, Papavassiliou E, Tarsy D, Shih LC. Phonemic verbal fluency decline after subthalamic nucleus deep brain stimulation does not depend on number of microelectrode recordings or lead tip placement. Parkinsonism Relat Disord 2014;20(4):400-404.

15. Costentin G, Derrey S, Gerardin E, et al. White matter tracts lesions and decline of verbal fluency after deep brain stimulation in Parkinson's disease. Hum Brain Mapp 2019;40(9):2561-2570.

16. Le Goff F, Derrey S, Lefaucheur R, et al. Decline in verbal fluency after subthalamic nucleus deep brain stimulation in Parkinson's disease: a microlesion effect of the electrode trajectory? J Parkinsons Dis 2015;5(1):95-104.

17. Okun MS. Deep-brain stimulation for Parkinson's disease. N Engl J Med 2012;367(16):15291538.

18. Troster Al, Jankovic J, Tagliati M, Peichel D, Okun MS. Neuropsychological Outcomes From Constant Current Deep Brain Stimulation for Parkinson's Disease. Movement Disord 2017;32(3):433440.

19. Bjerknes $S$, Toft $M$, Konglund $A E$, et al. Multiple Microelectrode Recordings in STN-DBS Surgery for Parkinson's Disease: A Randomized Study. Mov Disord Clin Prac 2018;5(3):296-305.

20. Giller $\mathrm{CA}$, Jenkins $\mathrm{P}$. Some technical nuances for deep brain stimulator implantation. Interdiscip Neurosur 2015;2(1):29-39. 
21. Jenkinson C, Fitzpatrick R, Peto V, Greenhall R, Hyman N. The Parkinson's Disease Questionnaire (PDQ-39): development and validation of a Parkinson's disease summary index score. Age Ageing 1997;26(5):353-357.

22. Lefaucheur R, Derrey S, Martinaud O, et al. Early verbal fluency decline after STN implantation: Is it a cognitive microlesion effect? Journal of the Neurological Sciences 2012;321(1-2):96-99.

23. Kocabicak E, Temel Y. Deep brain stimulation of the subthalamic nucleus in Parkinson's disease: surgical technique, tips, tricks and complications. Clin Neurol Neurosurg 2013;115(11):23182323.

24. Rey A. L'examen clinique en psychology [the psychological examination]. Paris: Presses Universitaires de Frances, 1958.

25. Lezak MD, Howieson DB, Loring DW. Neuropsychological Assessment. 4th ed. New York: Oxford University Press, 2004.

26. Stroop JR. Studies of interference in serial verbal reactions. Journal of Experimental Psychology 1935;18:643-662.

27. Reitan R. Trail-Making Test. Arizona: Reitan Neuropsychology Laboratory, 1979.

28. Corp. I. IBM SPSS Statistics for Windows, Version 24.0. . Armonk, NY: IBM Corp 2016.

29. Cernera S, Okun MS, Gunduz A. A Review of Cognitive Outcomes Across Movement Disorder Patients Undergoing Deep Brain Stimulation. Front Neurol 2019;10:419.

30. Soares C, Vilas-Boas MDC, Lopes EM, et al. Automated measures of gait dynamics and camptocormia angle in Parkinson's disease before and after subthalamic deep brain stimulation. Eur $\mathrm{J}$ Neurol 2019;26:844-844.

31. Shenai MB, Patel DM, Romeo A, et al. The Relationship of Electrophysiologic Subthalamic Nucleus Length as a Predictor of Outcomes in Deep Brain Stimulation for Parkinson Disease. Stereot Funct Neuros 2017;95(5):341-347.

32. Floden D, Matias C, Wathen C, Ozinga G, Hogue, Machado A. Contact location and non-motor outcomes in subthalamic nucleus Deep Brain Stimulation for Parkinson's disease. Movement Disord $2017 ; 32$.

33. Combs HL, Folley BS, Berry DT, et al. Cognition and Depression Following Deep Brain Stimulation of the Subthalamic Nucleus and Globus Pallidus Pars Internus in Parkinson's Disease: A Meta-Analysis. Neuropsychol Rev 2015;25(4):439-454.

34. Hojlund A, Petersen MV, Sridharan KS, Ostergaard K. Worsening of Verbal Fluency After Deep Brain Stimulation in Parkinson's Disease: A Focused Review. Comput Struct Biotechnol J 2017;15:6874.

35. Okun MS, Fernandez HH, Wu SS, et al. Cognition and mood in Parkinson's disease in subthalamic nucleus versus globus pallidus interna deep brain stimulation: the COMPARE trial. Ann Neurol 2009;65(5):586-595.

36. Miskin N, Thesen T, Barr WB, et al. Prefrontal lobe structural integrity and trail making test, part B: converging findings from surface-based cortical thickness and voxel-based lesion symptom analyses. Brain Imaging Behav 2016;10(3):675-685.

37. Varjacic A, Mantini D, Demeyere N, Gillebert CR. Neural signatures of Trail Making Test performance: Evidence from lesion-mapping and neuroimaging studies. Neuropsychologia 2018;115:78-87.

38. Shibuya-Tayoshi S, Sumitani S, Kikuchi K, et al. Activation of the prefrontal cortex during the Trail-Making Test detected with multichannel near-infrared spectroscopy. Psychiat Clin Neuros 2007;61(6):616-621.

39. Egner $\mathrm{T}$, Hirsch J. The neural correlates and functional integration of cognitive control in a Stroop task. Neuroimage 2005;24(2):539-547.

40. Planche V, Munsch F, Pereira $B$, et al. Anatomical predictors of cognitive decline after subthalamic stimulation in Parkinson's disease. Brain Struct Funct 2018;223(7):3063-3072. 


\section{Supplementary materials}

Supplementary Table 1. Overview of bilateral STN-DBS surgeries

\begin{tabular}{lccc}
\hline Year surgery & $\begin{array}{l}\text { Nr. of patients } \\
\text { operated }\end{array}$ & Included in study & \multicolumn{2}{c}{$\begin{array}{l}\text { Nr. of missing } \\
\text { subjects }\end{array}$} \\
\hline 2005 & 3 & 1 & 2 \\
2006 & 2 & 2 & 0 \\
2007 & 4 & 2 & 2 \\
2008 & 5 & 3 & 2 \\
2009 & 6 & 3 & 3 \\
2010 & 5 & 5 & 1 \\
2011 & 8 & 2 & 3 \\
2012 & 2 & 3 & 0 \\
2013 & 3 & 7 & 0 \\
2014 & 12 & 7 & 5 \\
2015 & 14 & 7 & 7 \\
2016 & 14 & 3 & 7 \\
Jan - March 2017 & 3 & 49 & 0 \\
\hline Total & 81 & & 32 \\
\hline
\end{tabular}

Supplementary Table 2. Reasons for exclusion

\begin{tabular}{lc}
\hline Reasons for exclusion & Nr. of cases \\
\hline Pre- and postoperative neuroimaging insufficient for determining the surgical & 12 \\
trajectory (bilaterally or unilaterally) & 16 \\
No available one year post neuropsychological evaluations* & 4 \\
Re-implantation/removal of electrodes due to complications & 32
\end{tabular}

${ }^{*}$ In most cases, there was available neuropsychological follow-up data. However, the follow-up duration was $>1$ year 



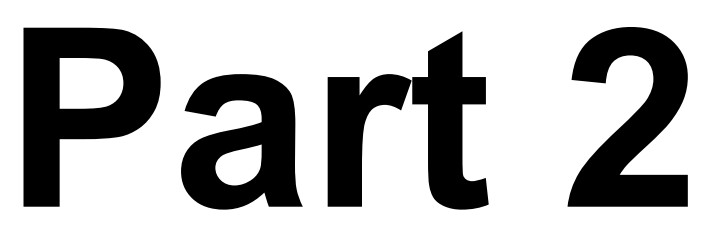

Mood symptoms in PD 



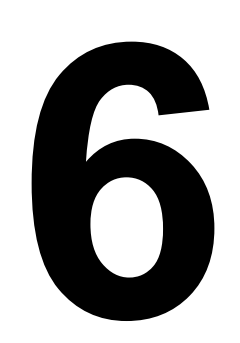

\section{Network analysis of symptoms in a Parkinson patient using experience sampling data: $A n n=1$ study}

Anne Mulders*, Rachel van der Velden*, Marjan Drukker,Mark Kuijf, Albert Leentjens Mov Disord 2018;33(12):1938-1944.

*These authors equally contributed to this work 


\section{ABSTRACT}

Background: around $50 \%$ of Parkinson's disease patients experience motor fluctuations after long-term treatment with levodopa. These fluctuations may be accompanied by mood fluctuations. Routine cross-sectional assessments cannot capture the extent of these motor and mood fluctuations and their possible associations. Experience sampling techniques that use frequently repeated measurements of symptoms over time are able to capture such fluctuations. Based on such data, longitudinal associations between symptoms can be studied using network analysis.

Aim: the purpose of this study is to identify longitudinal associations between motor symptoms and mood states in a patient with Parkinson's disease.

Methods: a 53-year old man with Parkinson's disease and motor fluctuations collected experience sampling data during 34 consecutive days. A set of dependent variables included tremor, rigidity, balance problems and 'on/off' state, and the mood variables anxiety, cheerful, and down. Independent variables were the same variables assessed at the preceding measurement. Regression coefficients were calculated and presented in a network graph.

Results: in this patient, anxiety and cheerfulness had a central position within the symptom network. Higher anxiety was prospectively associated with increased rigidity and tremor, and with feeling down. Cheerfulness was associated with less tremor. Balance problems were not influenced by cheerfulness nor anxiety, but increased balance problems were associated with reduced cheerfulness at the next assessment. Feeling down did not influence self-reported motor symptom severity at the next assessment.

Conclusion: this $n=1$ study shows that network analysis of experience sampling data may reveal longitudinal associations of self-reported motor symptoms and mood states that may have relevance for treatment strategies. 


\section{INTRODUCTION}

Up to $50 \%$ of patients with Parkinson's disease (PD) experience motor fluctuations after long-term treatment with levodopa ${ }^{1}$. Such fluctuations in motor symptoms are often accompanied by mood fluctuations ${ }^{2-4}$. Research involving mood fluctuations, their underlying mechanisms and their relationship with motor fluctuations has so far led to inconsistent results ${ }^{5}$. Insight into the timing and associations of fluctuations in motor and mood symptoms may provide information that can be valuable for clinical management. The frequency and severity of fluctuating symptoms are, however, difficult to capture in routine cross-sectional assessments, as such assessments use rating scales where a single assessment is considered representative for symptom severity over a longer time frame. Diary assessments can give more clarity, but are usually completed retrospectively at the end of the day or may hinder people from continuing their everyday activities. The Experience Sampling Method (ESM) is an attractive alternative to diary studies and cross-sectional assessments ${ }^{6}$. ESM includes multiple short, repeated, within-subject assessments at random moments during the day. These can include motor symptoms, and mood states as well as contextual information and, therewith, provide a good sample of subjective experiences. The ESM method originated in psychiatry, but has recently been applied in a number of other diseases, such as migraine, stroke and COPD $^{7-9}$. Broen and colleagues ${ }^{10}$ demonstrated that ESM is also a feasible method for the assessment of mood and motor symptoms in PD patients. When ESM data of a single subject are collected for a longer period of time, network analysis can be performed to study longitudinal relationships between symptoms. In network models, symptoms are conceptualised as mutually interacting, often reciprocally reinforcing elements in a complex dynamic system, also referred to as symptom networks ${ }^{11,12}$.

The aim of this $n=1$ study was to explore whether it is possible to identify longitudinal associations between motor symptoms and mood states in a single patient with PD suffering from motor fluctuations using experience sampling data and to generate a symptom network of these associations. We hypothesise that there is a temporal relationship between mood fluctuations and motor fluctuations, with negative emotions (down, anxiety) being associated with increased motor symptoms at the next assessment and positive emotions (cheerful) being associated with less motor symptom severity at the next assessment. 


\section{METHODS}

\section{Subject}

A 53-year old man with PD and motor fluctuations was referred to our hospital for a follow-up visit. The patient was diagnosed with tremor-dominant PD in 2011. Since then he was treated with levodopa/carbidopa. Currently, he takes a dose of $250 \mathrm{mg} 4$ times daily. In addition, he takes entacapone $200 \mathrm{mg} 4$ times and pramipexol $1.05 \mathrm{mg}$ once daily. He has been suffering from motor fluctuations since 2015. Initially these motor fluctuations, predominantly affecting tremor and rigidity, were predictable and involved levodopa-related 'wearing-off'. Later, the "on/off" fluctuations became unpredictable. The patient did not suffer from dyskinesia. Upon the start of ESM data collection the patient was cognitively intact and working part-time as a teacher. Baseline measurements are displayed in Table 1. The patient participated in ESM as part of his clinical assessment and agreed to have the period of data collection extended to one month. Informed consent was signed for the extended data collection and for publication of this $n=1$ study. ESM data was collected from March $16^{\text {th }}, 2017$, to April $18^{\text {th }}, 2017$.

\section{ESM data collection}

The ESM protocol in the present study is based on the routine outcome monitoring of the psychiatric outpatient department of our hospital, but with 5 Parkinson-specific items added: tremor, rigidity, walking problems, balance problems (all scored on a 7point Likert scale, ranging from 1= not at all to 7= very), and "on/off" state (dichotomous variable). This protocol was the same that was used earlier by Broen and colleagues ${ }^{10}$. The patient downloaded an ESM app, the Psymate ${ }^{T M}$ app [www.psymate.nl], on his own smartphone. Before data collection the patient received an oral briefing and a demonstration to ensure that the app and questionnaire were fully understood. The Psymate $^{\mathrm{TM}}$ app was programmed to emit a signal, a so-called "beep", 10 times daily at random moments in 90-minute time blocks, between 07.30 AM and 10.30 PM. After each beep, the patient was asked to fill out a 2-minute questionnaire that opened automatically after touching the phone and that consisted of 42 questions regarding his current mood state, the severity of his PD-related complaints, and his surroundings. The questions always appeared in the same order, and although it was not possible to skip single items before continuing to the next question, it was possible to turn back to previous questions and change the answer if necessary. To avoid recall bias, the 
questionnaires were only available for 15 minutes following the beep. After this timeframe, the patient received a notification regarding the missed questionnaire without the possibility to open and answer the questionnaire. The patient collected data for 34 consecutive days. Two days following the start and in the second week of ESM data collection, the patient was contacted by telephone to inquire about any difficulties or concerns regarding the ESM application and the questionnaire and to answer any additional questions the patient might have.

Table 1. Patient characteristics at baseline

\begin{tabular}{|c|c|}
\hline Measurement & Score / range \\
\hline Hoehn \& Yahr staging ${ }^{13}$ & $2.5 / 0-5$ \\
\hline UPDRS ${ }^{14}$ & $21 / 0-108$ \\
\hline Mini Mental State Examination ${ }^{15}$ & $28 / 0-30$ \\
\hline Parkinson Anxiety Scale ${ }^{16}$ & $17^{a} / 0-48$ \\
\hline Beck Depression Inventory-III7 & $14 / 0-63$ \\
\hline Parkinson's Disease Questionnaire- $8^{18}$ & $21^{b} / 0-31$ \\
\hline
\end{tabular}

\section{Statistical analyses}

Statistical analysis and graphical representations were performed using Stata $13^{19}$ and $\mathrm{R}^{20}$. The network analysis performed does not identify causal relationships, but merely temporal relationships that may or may not be causal. All data in this analysis are from a single participant. Therefore, the data do not have a multilevel structure and can be analysed with standard linear regression techniques, as opposed to group-level analyses in ESM studies ${ }^{21}$.

To assess variation over time, mean daily severity levels of mood and motor symptoms were plotted (Supplementary Figures $1 \mathrm{~A}$ and $1 \mathrm{~B}$, respectively). Floor and ceiling effects were checked using histograms (available on request). For the present analysis, the affective items cheerful and down, which reflect opposite poles, were a priori selected as representatives for positive and negative moods, respectively. The affective item anxiety was added based on the presence of clinically relevant anxiety at baseline. Tremor, rigidity and balance problems were selected for the analysis as 
the most disabling PD-related symptoms. The patient did not experience any gait problems or dyskinesia. Hence, these symptoms were not included in the analysis.

Six linear regression models were analysed with cheerful, down, anxiety, tremor, rigidity and balance problems as dependent variables. In addition, one logistic regression analysis was performed with 'on/off' state as dependent variable. The "off" state was patient rated and defined as the state in which he experienced no effect of anti-Parkinson medication. Because regression coefficients of linear and logistic regression cannot be combined in one network graph, the analysis with "on/off" as dependent variable was repeated with linear regression as has been done in a previous study $^{22}$. As the results, in terms of statistical significance, were very similar between the two analyses, the linear regression results were used to generate the network. Independent variables were the lag $(\mathrm{t}-1)$ of the same 7 variables added to the regression model simultaneously. In addition, a time variable was added to all regression models (consecutive number for beepcode to take into account time trends). This resulted in 49 regression coefficients. The first assessment of a new day, as well as assessments with a lag longer than 180 minutes from the previous assessment (i.e., when the participant missed 1 or more beeps) were excluded from the analysis. Given the exploratory nature of this study, no correction for multiple comparisons was performed.

Using the qgraph command in $\mathrm{R}$, the 49 regression coefficients were presented in a network graph. In this network, the nodes represent the variables and the connections between the nodes represent the regression coefficients. The thickness of the connections reflects the strength of the regression coefficient. In addition, indices of centrality were calculated using Excel (Microsoft, Redmond, Washington) and the qgraph package. In this context, "centrality" is an indicator of how central a specific variable is in the network. As in previous work, centrality indices are defined as follows: the outward strength is the sum of the connections from a specific node to all other nodes $^{23,24}$. The inward strength is the sum of the connections from all nodes to one specific node. The self-loop is the connection between a node at time point $\mathrm{t}-1$ and that same node at time point $t$. In both the inward and outward strengths, the self-loop is included. The sum of the outward and the inward strengths represents the node strength. Closeness, another measure of centrality, involves the sum of the length of 
the shortest paths between the node and all other nodes in the graph. Hence, the more central a node, the closer it is to all other nodes.

\section{RESULTS}

\section{ESM data}

During the course of 34 days, the patient received a total of 340 beeps. He answered 136 beeps, corresponding to a response rate of $40 \%$. There were some partial missing data as a result of uncompleted questionnaires, resulting in 121 completed assessments and a response rate of $36 \%$. The response rate declined during the course of ESM data collection and varied from $47 \%$ in week 1 to $29 \%, 46 \%, 36 \%$ and $19 \%$ in weeks 2, 3, 4 and 5, respectively. In 23 of the 121 assessments (19\%), the patient was in "off"-state. A total of 57 beep assessments were answered within 180 minutes of a previous beep assessment and were selected for the network analysis. The average time between beeps was $98.9 \min (S D=44.8$; range 15.6 - 176.7 minutes). Descriptive statistics are provided in Table 2. An example of fluctuations of included items during the course of a single day is plotted in Supplementary Figure 2. Except for a floor effect for anxiety, there were no strong floor effects and no ceiling effects.

Table 2. Descriptive statistics of ESM assessment of variables included in the analysis

\begin{tabular}{|c|c|c|c|c|c|}
\hline & $\mathbf{N}$ & Mean & $\begin{array}{l}\text { Standard } \\
\text { deviation }\end{array}$ & Minimum & Maximum \\
\hline \multicolumn{6}{|l|}{ Total } \\
\hline Cheerful & 121 & 5.0 & 0.49 & 3 & 6 \\
\hline Down & 121 & 3.0 & 1.03 & 1 & 5 \\
\hline Anxiety & 121 & 1.2 & 0.66 & 1 & 5 \\
\hline Tremor & 121 & 1.9 & 0.87 & 1 & 6 \\
\hline Rigidity & 121 & 1.7 & 0.90 & 1 & 6 \\
\hline Balance problems & 121 & 1.4 & 0.71 & 1 & 5 \\
\hline On/off state, \% & 121 & $81 \%$ on time & n.a. & n.a. & n.a. \\
\hline \multicolumn{6}{|l|}{ Network analysis } \\
\hline Cheerful & 57 & 4.9 & 0.52 & 3 & 6 \\
\hline Down & 57 & 3.0 & 1.08 & 1 & 5 \\
\hline Anxiety & 57 & 1.1 & 0.54 & 1 & 5 \\
\hline Tremor & 57 & 1.8 & 0.93 & 1 & 6 \\
\hline Rigidity & 57 & 1.7 & 1.03 & 1 & 6 \\
\hline Balance problems & 57 & 1.5 & 0.87 & 1 & 5 \\
\hline On/off state, \% & 57 & $88 \%$ on time & n.a. & n.a. & n.a. \\
\hline
\end{tabular}




\section{Network graph}

Cheerfulness and anxiety had central positions within the symptom network (Figure 1). A network graph displaying the significant associations only is plotted in Supplementary Figure 3. Anxiety was associated with higher scores for tremor $(B=0.61, p=0.001)$ and rigidity $(B=0.47, p=0.013)$ at the next assessment, as well as with feeling less cheerful $(B=-0.40, p=0.037)$ and more down $(B=0.52, p=0.003)$. Cheerful was associated with lower scores for tremor $(B=-0.55, p=0.002)$ and rigidity $(B=-0.38, p=0.037)$ at the next assessment. Balance problems were not predicted by cheerfulness $(B=0.05, p=0.78)$ or anxiety, but instead more balance problems led to lower scores on cheerful at the next assessment $(B=-0.50, p=0.011)$. In contrast, feeling down did not influence motor symptom severity at the next assessment, or vice versa. "On" and "off" states could not be predicted by motor symptoms, but anxiety was associated with "off" at the next assessment $(B=-0.37, p=0.024)$. In addition to associations between different variables, self-loops were created that reflected the relationship between the severity of the same symptom at the next assessment. The self-loops cheerful $(B=-0.66, p=0.001)$, anxiety $(B=0.59, p=0.000)$, down $(B=0.38$, $p=0.006)$ and balance problems $(B=0.58, p=0.003)$ were statistically significant.

Centrality indices are provided in Table 3.Anxiety had the highest node strength with a value of 4.79 , followed by cheerful with a value of 4.42 . "On/off" state had the lowest node strength, with a value of 1.77. Closeness was highest for anxiety, cheerful and for balance problems, indicating that those 3 nodes were closest to all other nodes in terms of strength.

Table 3. Centrality indices

\begin{tabular}{lcccc}
\hline & Closeness & $\begin{array}{c}\text { Inward } \\
\text { strength }\end{array}$ & $\begin{array}{c}\text { Outward } \\
\text { strength }\end{array}$ & $\begin{array}{c}\text { Node } \\
\text { Strength }\end{array}$ \\
\hline Cheerful & 0.038 & 1.90 & 2.51 & 4.42 \\
Down & 0.028 & 1.94 & 1.27 & 3.21 \\
Anxiety & 0.048 & 1.73 & 3.05 & 4.79 \\
Tremor & 0.017 & 1.72 & 0.48 & 2.20 \\
Rigidity & 0.024 & 1.40 & 0.96 & 2.37 \\
Balance problems & 0.036 & 0.97 & 1.65 & 2.62 \\
On/off state & 0.022 & 1.00 & 0.77 & 1.77 \\
\hline
\end{tabular}




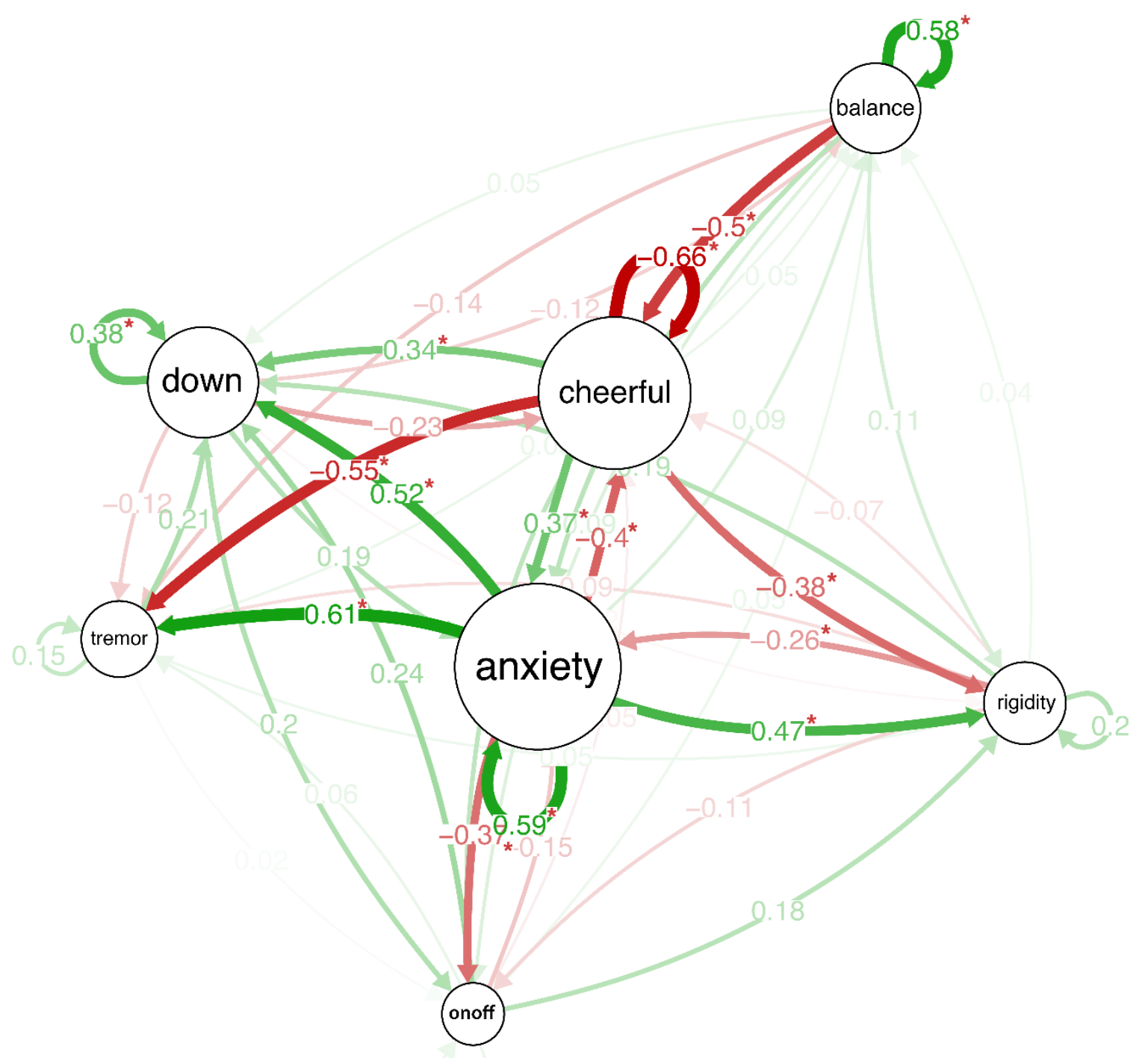

Figure 1. Symptom network

Network graph displaying prospective associations between the mood states 'cheerful', 'down' and 'anxiety', and Parkinsonian symptoms (tremor, rigidity, balance problems and on/off state). Negative regression coefficients are red, positive coefficients green. Significant coefficients are marked as tick lines with an asterix. The position of the variables within the graph is a measure of centrality.

\section{DISCUSSION}

\section{Interpretation of the results}

With this $n=1$ study we showed that network analysis of ESM data can be used to prospectively reveal associations between motor and mood states in PD. In the symptom network of this patient, the variables of anxiety and cheerful appeared to play a more important role than the variable down because they had stronger associations 
with several motor symptoms and had the highest node strength and closeness index. The centrality of mood variables in the symptom network indicates that affect plays an important role in the way motor symptoms are experienced and reported and even predict symptom severity prospectively. Whereas most studies in PD have focused on the effect of (fluctuations in) motor symptoms on mood, this finding shows that it is as important to understand the role of psychological factors and mood states in predicting self-reported symptoms in PD.

"On/off" state had the lowest node strength, corresponding to the finding that "on/off" state could not be predicted from motor symptoms or affective states and appears to be relatively independent from other variables, except for anxiety. This finding is consistent with the notion that motor fluctuations usually start as end-of-dose deterioration, but tend to become random and unpredictable in the later course of the disease, as was the case with our patient. This unpredictability is also reflected by the fact that the variable "on/off" does not have a self-loop in the network graph. As a general comment, based on the outcome in this patient, it may be worthwhile to study the role of anxiety in predicting "off" states.

Despite the data not being multilevel, there can be autocorrelation between consecutive assessments or between assessments on the same day; the shorter the time between 2 assessments, the higher the correlation. The regression coefficients of the balance problems and cheerful self-loops indicate high autocorrelation. The negative regression coefficient in cheerful $(-0.66)$ is surprising and one can only speculate about potential explanations. It may be that the already high score on cheerfulness reduces the chance of scoring even higher at a next assessment. While the variable time was included in the analysis, it was not possible to further control for autocorrelation as only 1 patient was included.

\section{Data quality}

The Psymate ${ }^{\mathrm{TM}}$ app is available as an app for smartphones or other electronic devices, such as iPods. The patient opted for downloading the app on his own smartphone rather than using one of the available iPods. Previous studies have not shown any difference in compliance between the 2 devices ${ }^{10}$. For this patient, the response rate was $36 \%$, which is above the $33 \%$ generally considered valid for analysis ${ }^{21,25}$. Because 
it was not possible to skip single items in the questionnaire, thereby preventing selective missingness, all missing values were solely the result of missed beeps and incidentally by not finishing a questionnaire. Missed beeps can be mostly explained from an inability to answer questionnaires as a result of other activities, such as being in traffic or working. However, it also reflects that collecting ESM data during a longer period of time can be burdensome, resulting in lower response rates over time as observed in this patient. The patient was contacted once weekly during the first 2 weeks and at the end of data collection. As the response rate was still sufficient during the first 2 weeks, motivational issues were not addressed. A lack of motivation might have contributed to the low response rate in the final weeks of data collection. Regular weekly contact during ESM data collection might increase response rates, especially when data are being collected for a longer time frame. In future studies, it might also be worthwhile to evaluate the patient's experience with ESM in order to get more insight into compliance ${ }^{10}$.

During the last 2 weeks of data collection, the patient reported stable levels of cheerfulness (Likert score 5) and less fluctuations in motor symptoms. One could argue this created bias and that the patient only responded to beep assessments when feeling cheerful or refrained from answering questions during periods with severe motor symptoms. However, during the same period of data collection, there was a high degree of variation in down, which makes cheerfulness bias unlikely. Also, the higher response rate in the first period, during which the patient reported varying levels in motor symptom severity, ranging from 1 to 6 , shows that experiencing more severe symptoms did not lead to reduced response. The present data do include various beeps where the patient is in the "off" state. Therefore, we expect that the network is representative for a large proportion of the time.

\section{Methodological issues}

To our knowledge, this is the first study to use long-term ESM data collected in a single patient with PD to study longitudinal associations between motor and mood symptoms. This exploratory study has, however, several limitations. First, the response rate of $36 \%$ was low. Earlier ESM studies suggest that at least $33 \%$ of the assessments need to be completed for their data to be considered valid ${ }^{21} 25,26$. Preferably, prospective associations are studied between 2 successive beep assessments. As this would 
result in too few usable assessments, assessments were considered valid if they were within 180 minutes of a previous beep, regardless of whether there was a missed beep in between. Although the resulting selection of 57 couples of beep assessments for the network analysis, of which 7 in "off" phase, does not influence the representativeness of the data, it does result in lower power. Because of the reduced response rate in the last weeks of data collection as well as the more stable scores on cheerfulness in that period, we performed a sensitivity analysis based on the data of the first 20 days of sampling, which resulted in a very similar network (see Supplementary Figure 4). Second, although we looked at node strength and closeness as measures of centrality, betweenness as measure for centrality was not determined for this study. Bak and colleagues $^{23}$, who used network analysis in a patient with recurrent psychosis, showed that the importance and clinical relevance of betweenness as an indicator of network performance is low in this type of analysis. In their analysis, as well as in ours, all paths between variables are considered important and the length of various paths can be very similar, whereas betweenness only reflects the shortest path between variables. Third, when performing network analysis including lags, there is a time axis in the analysis. However, even with the time axis only temporal associations can be shown and it does not indicate causality. Finally, the patient did not collect more objective measures of motor functioning.

\section{Clinical implications}

The ultimate aim is to provide patients with optimal and personalised care to reduce their suffering and increase their well-being. ESM can be used for this purpose by generating treatment-relevant information specific for that patient that cannot be obtained by commonly used cross-sectional assessment methods and rating scales. Previous studies using network analysis for different diseases have demonstrated that ESM data can be incorporated into a personalised treatment plan and facilitate decision-making in a collaborative process ${ }^{21}$, 23, 27. For PD patients, ESM data can provide insight into the relationship between different variables and symptom severity. This particular network analysis focused on mood variables, but based on the specific situation of the patient, other relevant variables can be included in the network. Contextual variables, such as present company, whereabouts and activities, can provide information on whether symptoms worsen or improve in specific situations. This information can be valuable for both patients and physicians or other members of 
the patient's health care team, as it may give more insight into determinants of the patient's symptoms and may lead to (non-pharmacological) treatment recommendations. For example, when affective states or contextual factors appear to play a central role in self-perceived motor symptom severity, it may be preferable to treat the mood state or to improve coping with the specific situation. Based on the network analysis of ESM data in the present study, our patient was offered individualised face-to-face feedback regarding the specific connections in his symptom network, and in particular regarding the possible role of positive emotions in improving self-perceived motor symptoms. However, the patient preferred to receive a written report of the results as he was limited in his time. We do not know whether a lack of motivation contributed to his decision of not desiring a face-to-face meeting or whether the patient benefited from the provided information. For future studies it would be interesting to use ESM data sampling to develop a treatment plan with personalised interventions and to use post-intervention ESM data sampling to study the effectiveness of the intervention. It may also be valuable to combine ESM with downloadable apps or wearable devices that are able to objectively quantify motor symptom severity, such as severity of tremor or poverty of movement. This is technically feasible and will provide information that, when combined with ESM, will allow comparison between the objectively measured severity of the tremor, and the subjectively experienced severity of tremor ${ }^{28}$. Integrating these measurements could then be useful for fine-tuning future therapies such as closed loop deep brain stimulation systems ${ }^{29}$.

\section{CONCLUSION AND RECOMMENDATIONS}

In this $n=1$ study, we showed that network analysis of ESM data can be used to sample moment-to-moment variability and to prospectively reveal relevant associations between motor and mood variables in an individual patient. These prospective associations may be different for different patients. When network patterns in a single patient can be replicated in other PD patients, this may lead to more general treatment recommendations. To reveal such network patterns, multiple $n=1$ studies with extensive data collection may be superior to ESM studies that include more patients but collect data for a briefer period of time. More research into the usefulness of ESM as a therapeutic tool is warranted. 


\section{REFERENCES}

1. Fox SH, Lang AE. Levodopa-related motor complications--phenomenology. Mov Disord 2008;23 Suppl 3:S509-514.

2. Witjas T, Kaphan E, Azulay JP, et al. Nonmotor fluctuations in Parkinson's disease: frequent and disabling. Neurology 2002;59(3):408-413.

3. Storch A, Schneider CB, Wolz M, et al. Nonmotor fluctuations in Parkinson disease: severity and correlation with motor complications. Neurology 2013;80(9):800-809.

4. Hillen ME, Sage JI. Nonmotor fluctuations in patients with Parkinson's disease. Neurology 1996;47(5):1180-1183.

5. Martinez-Fernandez R, Schmitt E, Martinez-Martin P, Krack P. The hidden sister of motor fluctuations in Parkinson's disease: A review on nonmotor fluctuations. Mov Disord 2016;31(8):10801094.

6. Palmier-Claus JE, Myin-Germeys I, Barkus E, et al. Experience sampling research in individuals with mental illness: reflections and guidance. Acta Psychiatr Scand 2011;123(1):12-20.

7. Odawara M, Hashizume M, Yoshiuchi K, Tsuboi K. Real-Time Assessment of the Effect of Biofeedback Therapy with Migraine: A Pilot Study. Int J Behav Med 2015;22(6):748-754.

8. Jean FA, Swendsen JD, Sibon I, Feher K, Husky M. Daily life behaviors and depression risk following stroke: a preliminary study using ecological momentary assessment. J Geriatr Psychiatry Neurol 2013;26(3):138-143.

9. Mulvaney SA, Ho YX, Cala CM, et al. Assessing adolescent asthma symptoms and adherence using mobile phones. J Med Internet Res 2013;15(7):e141.

10. Broen MP, Marsman VA, Kuijf ML, Van Oostenbrugge RJ, van Os J, Leentjens AF. Unraveling the Relationship between Motor Symptoms, Affective States and Contextual Factors in Parkinson's Disease: A Feasibility Study of the Experience Sampling Method. PLoS One 2016;11(3):e0151195.

11. van Borkulo C, Boschloo L, Borsboom D, Penninx BW, Waldorp LJ, Schoevers RA. Association of Symptom Network Structure With the Course of [corrected] Depression. JAMA Psychiatry 2015;72(12):1219-1226.

12. Borsboom D, Cramer AO. Network analysis: an integrative approach to the structure of psychopathology. Annu Rev Clin Psychol 2013;9:91-121.

13. Goetz CG, Poewe W, Rascol O, et al. Movement Disorder Society Task Force report on the Hoehn and Yahr staging scale: status and recommendations. Mov Disord 2004;19(9):1020-1028.

14. Movement Disorder Society Task Force on Rating Scales for Parkinson's Disease. The Unified Parkinson's Disease Rating Scale (UPDRS): status and recommendations. Mov Disord 2003;18(7):738750.

15. Folstein MF, Folstein SE, McHugh PR. "Mini-mental state". A practical method for grading the cognitive state of patients for the clinician. J Psychiatr Res 1975;12(3):189-198.

16. Leentjens AF, Dujardin K, Pontone GM, Starkstein SE, Weintraub D, Martinez-Martin P. The Parkinson Anxiety Scale (PAS): development and validation of a new anxiety scale. Mov Disord 2014;29(8):1035-1043.

17. Beck AT, Steer RA, Ball R, Ranieri W. Comparison of Beck Depression Inventories -IA and -II in psychiatric outpatients. J Pers Assess 1996;67(3):588-597.

18. Jenkinson C, Fitzpatrick R, Peto V, Greenhall R, Hyman N. The PDQ-8: development and validation of a short-form Parkinson's disease questionnaire. Psychology and Health 1997;12(6):805814.

19. StataCorp. Stata Statistical Software: Release 13. College Station, TX: StataCorp LP 2013.

20. Team R. R: A Language and Environment for Statistical Computing. Vienna, Austria: Computing RFfS 2013.

21. Verhagen SJ, Hasmi L, Drukker M, van Os J, Delespaul PA. Use of the experience sampling method in the context of clinical trials. Evid Based Ment Health 2016;19(3):86-89.

22. Klippel A, Viechtbauer W, Reininghaus U, et al. The Cascade of Stress: A Network Approach to Explore Differential Dynamics in Populations Varying in Risk for Psychosis. Schizophr Bull 2017. 
23. Bak M, Drukker M, Hasmi L, van Os J. An $n=1$ Clinical Network Analysis of Symptoms and Treatment in Psychosis. PLoS One 2016;11(9):e0162811.

24. Hasmi L, Drukker M, Guloksuz S, et al. Network Approach to Understanding Emotion Dynamics in Relation to Childhood Trauma and Genetic Liability to Psychopathology: Replication of a Prospective Experience Sampling Analysis. Front Psychol 2017;8:1908.

25. Delespaul P. Assessing Schizophrenia in Daily Life. The Experience Sampling Method. . Maastricht: Maastricht University Press 1995.

26. Kimhy D, Delespaul P, Corcoran C, Ahn H, Yale S, Malaspina D. Computerized experience sampling method (ESMc): assessing feasibility and validity among individuals with schizophrenia. $J$ Psychiatr Res 2006;40(3):221-230.

27. Kroeze R, van der Veen DC, Servaas MN, et al. Personalized feedback on symptom dynamics of psychopathology: A proof-of-principle study. Journal of Person-Orientated Research 2017;3(1):1-10. 28. Kubben PL, Kuijf ML, Ackermans LP, Leentjes AF, Temel Y. TREMOR12: An Open-Source Mobile App for Tremor Quantification. Stereotact Funct Neurosurg 2016;94(3):182-186.

29. Arlotti M, Rosa M, Marceglia S, Barbieri S, Priori A. The adaptive deep brain stimulation challenge. Parkinsonism Relat Disord 2016;28:12-17. 


\section{Supplementary materials}

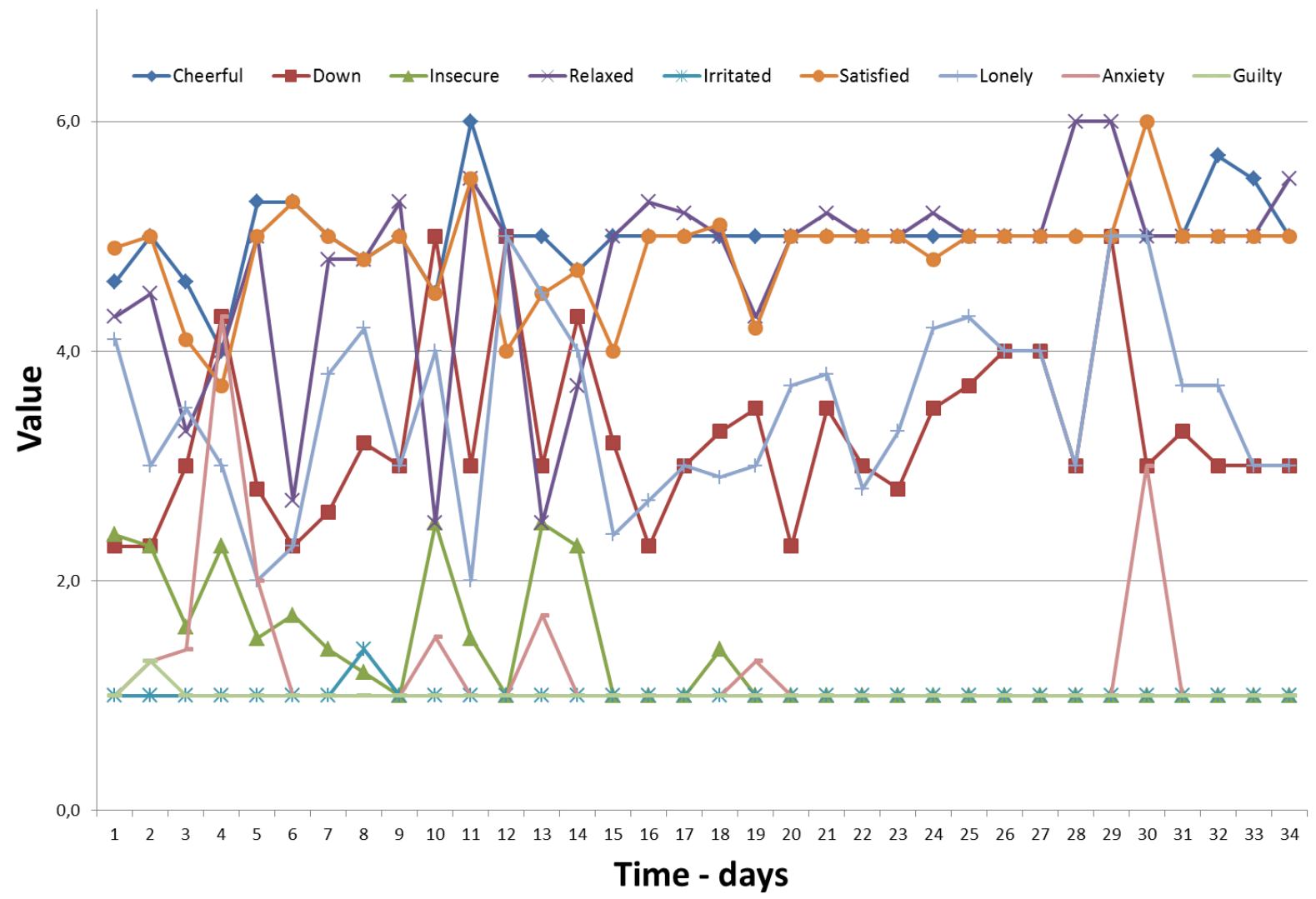

Supplementary Figure 1A. Mean daily severity levels per affective item per day for the total period of ESM data collection (note: these are mean scores and items scores may still fluctuate over the respective day).

Items were scored on a 7 -point likert scale (ranging from $1=$ not at all to $7=$ very). 


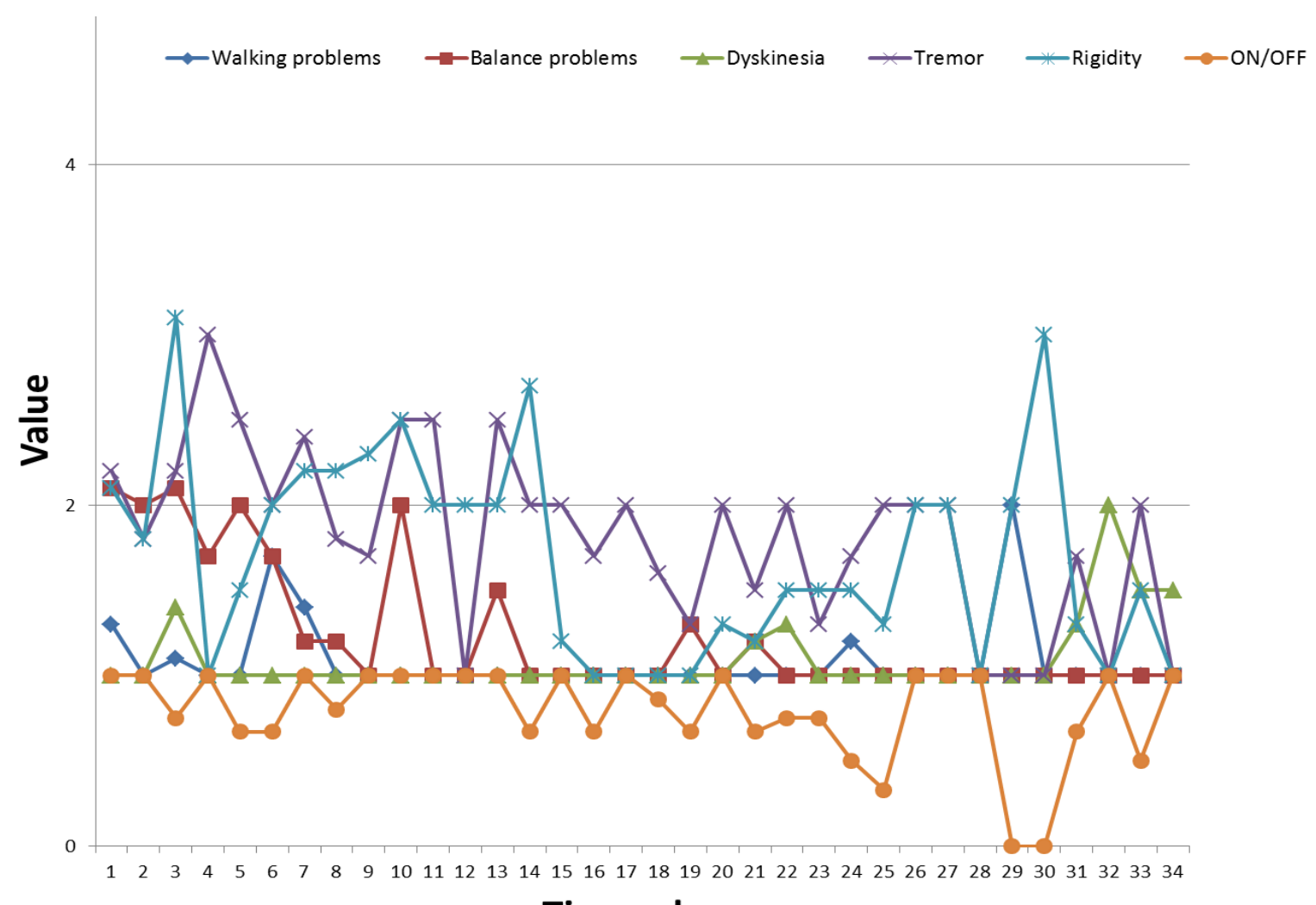

Time - days

Supplementary Figure 1B. Mean daily severity levels per motor item per day for the total period of ESM data collection (note: these are mean scores and items scores may still fluctuate over the respective day).

Items were scored on a 7 -point likert scale (ranging from $1=$ not at all to $7=$ very). For ON/OFF state, the values 1 and 0 represent 'ON' state and 'OFF' state, respectively. 


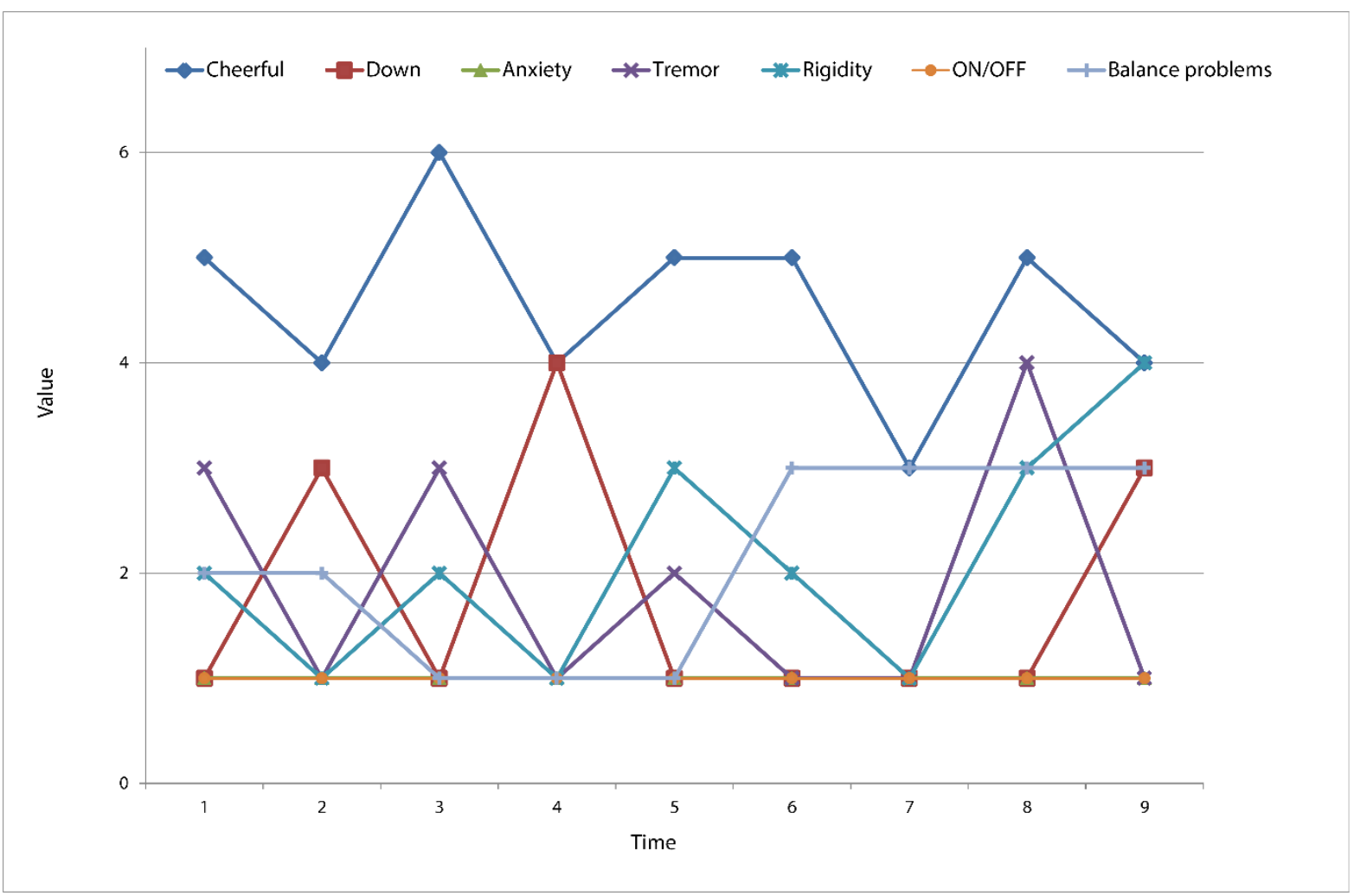

Supplementary Figure 2. ESM scores per selected item for one day.

Items were scored on a 7-point likert scale (ranging from $1=$ not at all to $7=$ very). For ON/OFF state, the values 1 and 0 represent 'ON' state and 'OFF' state, respectively. The patient completed 9 questionnaires (beep assessments). 


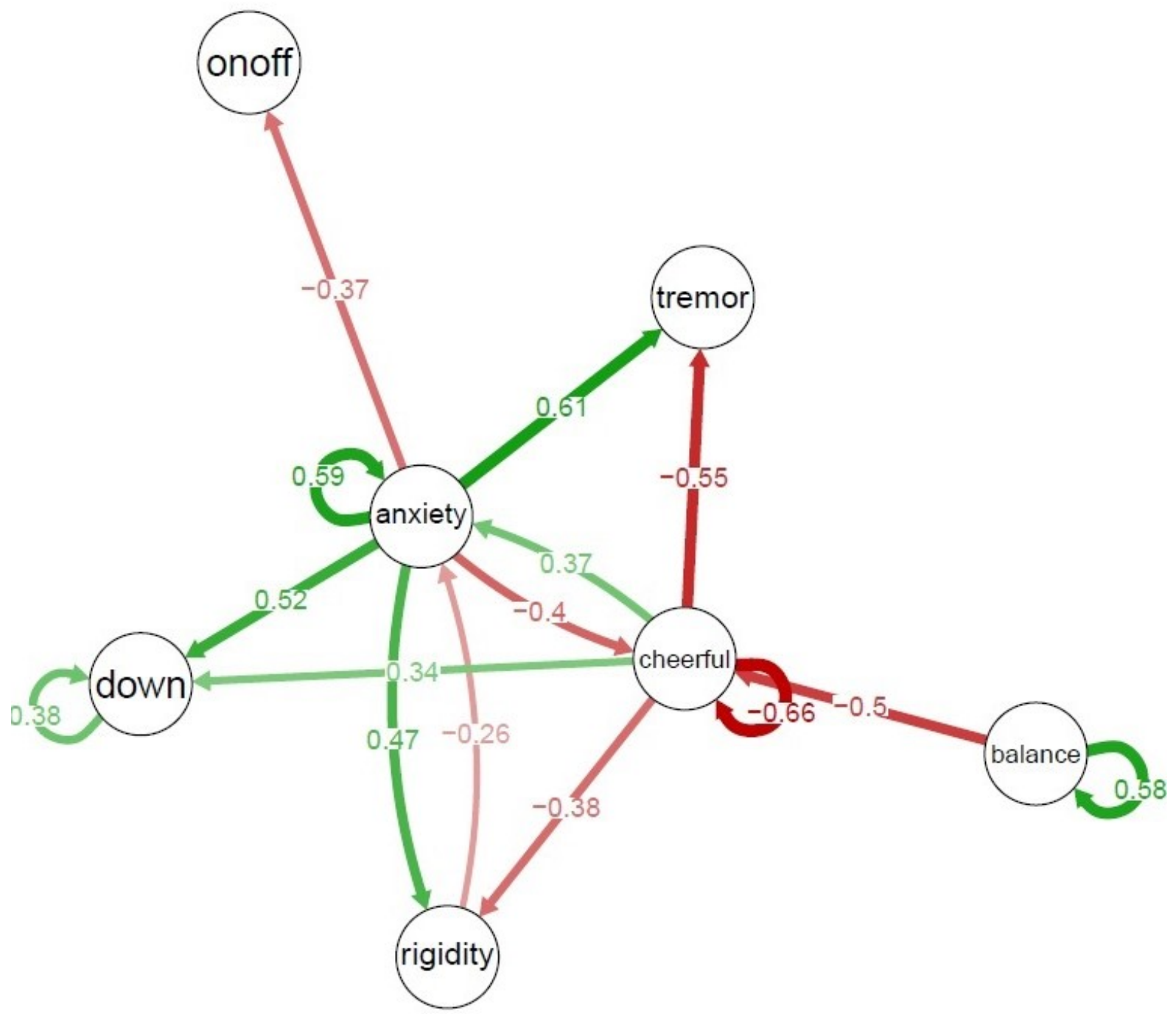

Supplementary Figure 3. Network graph of significant associations.

Network graph displaying prospective significant associations between the mood states 'cheerful', 'down' and 'anxiety', and Parkinsonian symptoms (tremor, rigidity, balance problems and on/off state). Negative regression coefficients are red, positive coefficients green. Significant coefficients are marked as tick lines with an asterix. The position of the variables within the graph is a measure of centrality. 


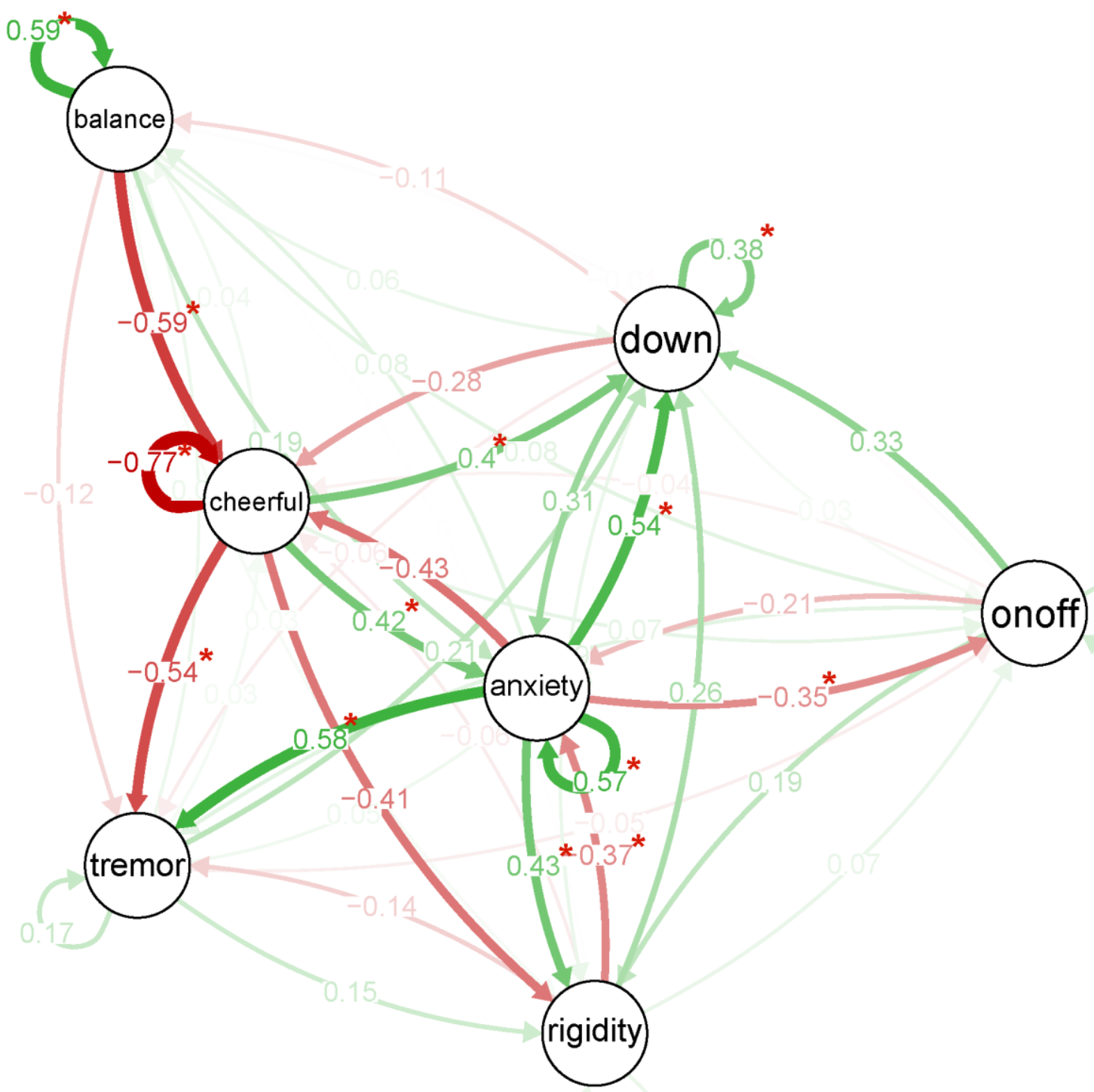

Supplementary Figure 4. Sensitivity analysis network graph.

Network graph of the sensitivity analysis of the first 20 days of ESM data collection displaying prospective associations between the mood states 'cheerful', 'down' and 'anxiety', and Parkinsonian symptoms (tremor, rigidity, balance problems and on/off state). Negative regression coefficients are red, positive regression coefficients green. Significant coefficients are marked as thick lines with an asterix. The position of the variables within the graph is a measure of centrality. 



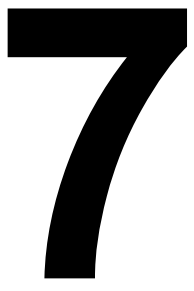

\section{Usability of the experience sampling method in Parkinson's disease on a group and individual level}

Anne Mulders*, Rachel van der Velden*, Marjan Drukker, Martijn Broen, Mark Kuijf, Albert Leentjens

Mov Disord 2020; 35(7): 1145-1152.

*These authors equally contributed to this work 


\section{ABSTRACT}

Background: around $50 \%$ of Parkinson's disease patients experience motor fluctuations, which are often accompanied by mood fluctuations. The nature of the relationship between motor and mood fluctuations remains unknown. It is suggested that the Experience Sampling Method (ESM) can reveal such associations on both a group and individual level. Revealing group patterns may enhance our understanding of symptom interactions and lead to more general treatment recommendations, while analyses in individual patients can be used to establish a personalised treatment plan.

Objectives: to explore the usability of routinely collected ESM data over a brief period of time to detect associations between motor fluctuations, affective state, and contextual factors in Parkinson's disease patients with motor fluctuations on a group level and on an individual level.

Methods: eleven patients with motor fluctuations collected data at 10 semi-random moments over the day for five consecutive days.

Results: on a group level, multilevel analyses showed significant associations between all motor symptoms and positive affect. Being at home was associated with increased balance problems and rigidity. Analyses on an individual level revealed much less significant associations that mostly, but not always, were in line with the results on a group level.

Conclusion: this exploratory study showed significant associations between affective state, motor symptoms and contextual factors in a group of Parkinson's disease patients with motor fluctuations, but less so in individual patients. Since the ultimate aim is to use ESM as an aid to personalise treatments, the sensitivity of the approach needs to be increased. 


\section{INTRODUCTION}

The cornerstone of treatment for Parkinson's disease (PD) is levodopa replacement ${ }^{1}$. Unfortunately, fluctuations in parkinsonian symptoms due to levodopa replacement may occur sometimes as early as a few months after initiation of levodopa treatment ${ }^{2}$. These usually start with 'end-of-dose wearing-off', but eventually, as the disease progresses, lead to unpredictable fluctuations between 'ON' and 'OFF' state in approximately $50 \%$ of patients ${ }^{2}, 3$. The presence of motor fluctuations is associated with lower quality of life and accounts for significant morbidity ${ }^{4-6}$. Motor fluctuations are often accompanied by distressing mood fluctuations, such as fluctuations in anxiety or depressive symptoms ${ }^{7-9}$. Due to the fluctuating nature of motor and mood symptoms their frequency is difficult to capture in routine-cross sectional assessments that are usually retrospective in nature 10-12. The Experience Sampling Method (ESM) can overcome these limitations ${ }^{13,14}$. With ESM, experiences and behaviours as well as moment-to-moment changes in physical and mental states, and contextual factors in the subject's natural environment can be sampled. Data are collected repeatedly and randomly during the day, for several days, thereby reducing recall bias ${ }^{11,15,16}$. In a recent $\mathrm{N}=1$ study we demonstrated that ESM data collected for one month is able to reveal longitudinal associations between motor symptoms and mood states, and has the potential to be used as an aid to optimise individual treatment ${ }^{14,17}$. However, in routine patient care much briefer sampling periods of 5 to 7 days are commonly used, since longer periods of time are considered burdensome for patients ${ }^{14}$. The question is whether brief periods of data collection generate enough power for the identification of significant associations between mood and motor symptoms. Since the aim is to develop ESM as an aid to personalise treatment in individual patients, adequate power needs to be ensured.

The aim of the present study was to explore the usability of ESM data routinely collected over a brief period of time in PD patients suffering from motor fluctuations, and to explore the sensitivity to detect associations between affective state, contextual factors, motor symptoms and motor state on both a group and individual level. For that purpose, we explored: (1) associations between affective state, motor symptoms and contextual factors in PD on a group level, as well as the role of motor (ON/OFF) state in these associations; and (2) whether ESM data are sensitive enough to reveal similar associations in individual patients. 


\section{METHODS}

\section{Design and participants}

This is a cross-sectional observational study. Twelve PD patients with motor fluctuations were recruited from the Movement Disorder clinic of Maastricht University Medical Centre (MUMC+). Five of these were recruited in January and February 2015 and participated as part of a feasibility study of ESM in PD patients ${ }^{11}$. Seven patients were additionally recruited between February 2017 and April 2017. Inclusion criteria were: a diagnosis of idiopathic PD according to the Queen Square Brain Bank diagnostic criteria ${ }^{18}$, the usage of a stable dose of levodopa or other antiparkinsonian medication for at least one month, the presence of motor fluctuations as indicated by part IV of the Unified Parkinson's Disease Rating Scale (UPDRS), and an age between 50 and 80 years. Subjects with cognitive impairment, operationalised as a Mini-Mental State Examination (MMSE) $<26^{19}$, were excluded in order to avoid problems with working with the mobile ESM application. Other exclusion criteria were: the presence of neurological disorders other than PD and excessive use or abuse of alcohol, drugs or benzodiazepines. The institution's Medical Ethics Committee ruled that collection of ESM data in the context of routine clinical outcome monitoring is exempt from ethical approval.

\section{Assessment}

After informed consent was obtained, all patients underwent a baseline assessment which involved the collection of demographic and disease-related characteristics. All patients were tested in ON state. The following instruments and scales were administered: the Hoehn and Yahr staging scale ${ }^{20}$, the UPDRS part $1 \mathrm{II}^{21}$ to quantify motor symptoms, the MMSE ${ }^{19}$ to assess global cognitive function, the Parkinson Anxiety Scale (PAS) ${ }^{22}$ to assess anxiety symptoms, and the Beck Depression Inventory II (BDI-II) ${ }^{23}$ to assess depressive symptoms. Quality of life was assessed using the Parkinson Disease Questionnaire-824. The UPDRS-III was administered by a neuropsychologist holding an MDS-issued certificate of training in application of the UPDRS and ample experience in administering this instrument (AM). All other scales were administered by a trained medical student (Rv/dV).

Using a mobile device with an ESM application (PsyMate ${ }^{T M}$, www.psymate.eu), participants were asked to answer several questions multiple times a day. During the 
baseline assessment subjects received oral information and a demonstration to ensure that the ESM app was fully understood. Subjects were given the option to either install the ESM app on their own smartphone or to use an iPod-touch with the PsyMate ${ }^{T M}$ app provided by MUMC for the duration of the study. The following five consecutive days, the PsyMate ${ }^{\mathrm{TM}}$ generated auditory reminders (beeps) at unpredictable moments in each of ten 90-minute time blocks between 7:30am and 10:30pm (10 beeps a day, during five days). After each reminder, subjects were asked to fill out a set of questions regarding motor, mood and general symptoms, as well as contextual factors (see ESM questions). Questions always appeared in the same order and were available for 15 minutes only in order to reduce recall bias. Answering the questionnaires takes approximately two minutes. In addition, every morning and evening subjects were asked to fill out a morning and evening questionnaire, consisting of questions about the previous night and day, respectively.

Two days following the start of data collection, participants were contacted by telephone to inquire about any difficulties or concerns regarding the ESM application and questionnaires, and to solve any problems if necessary. Five days following the start of data collection, the data was uploaded in an anonymous central database and subjects returned the Ipod-touch, if necessary.

\section{ESM questions}

The questions in the ESM protocol were based on a protocol used for routine outcome monitoring of the psychiatric outpatient department of our hospital. The protocol includes questions in several domains: context (e.g. 'Where am I?'), events ('the most important event since the last beep was'), mood (e.g. 'I feel happy'), and somatic (e.g. 'I am tired'). The protocol was supplemented with 6 Parkinson-specific items (motor symptoms): 'tremor', 'rigidity', walking problems', 'balance problems', 'dyskinesia' (all scored on a 7-point Likert scale, ranging from 1=not at all to 7=very much), and 'ON/OFF state' $\left(\mathrm{ON}=1\right.$, OFF=0) (supplementary table 1) ${ }^{11}$. 'ON' and 'OFF' states are defined subjectively by each individual subject. In general, in an 'ON' state the subject perceives his antiparkinsonian medication to be working properly in alleviating symptoms, while in an 'OFF' state symptoms start to reappear. Based on standard ESM mood items (also scored on a 7-point Likert scale), two mood-related variables reflecting positive and negative affect were computed. Positive affect was defined as 
the mean score of the items 'cheerful', 'relaxed' and 'satisfied'. Negative affect was defined as the mean score of the items 'insecure', 'irritated', 'lonely', 'anxious', 'down', and 'guilt'. Based on the questions 'Where am I?' and 'With who am l', two contextual variables 'at home' (at home $=1$, not at home $=0$ ) and 'being alone' (alone $=1$, not alone $=0$ ) were computed.

\section{Statistical analysis}

All statistical analyses were performed using SPSS version 24.0 for Windows ${ }^{25}$ and Stata version $13^{26}$. Descriptive analyses were conducted to assess response rates. To assess usability on a group level, associations between affective state, contextual factors, motor symptoms and ON/OFF state were studied in the full data set. ESM data have a hierarchical structure due to within-subject clustering: repeated measurements (level 1) are nested within individuals (level 2). Nesting of data violates the assumption of independent residuals, since observations within subjects are more similar than observations among different subjects. Therefore, multilevel linear regression analyses were performed using the Stata mixed command with subject as the macro level. The following motor symptoms were the dependent variables in the regression models: 'tremor', 'rigidity', 'walking problems', 'balance problems' and 'dyskinesia'. To control for time trends, a time variable was added to all regression models (beepcode=daynumber*10+beepnumber). Independent variables were positive and negative affect, as well as being at home and being alone.

First, in order to assess whether ON/OFF state is a modifier in the association between affective state and contextual factors on the one hand and motor symptoms on the other hand, interaction effects of all independent variables with ON/OFF state (ON = 1 , OFF $=0$ ) were analysed including each independent variable in a separate model. Importantly, convergence was not achieved after including random slopes of the independent variable, ON/OFF state and beepcode, and consequently, only models including a random slope for beepcode were run. As this results in too optimistic pvalues, permutation testing was performed in case of a statistically significant result. Permutation testing obtains the distribution of regression coefficients under the null hypothesis. To obtain $>1000$ valid $p$-values, 1100 permutation tests were performed. For this, the dependent variable was removed from the data and within-subject randomly reshuffled and merged to the original data, while keeping the multilevel 
Table 1. Demographic and disease-related characteristics of participating patients

\begin{tabular}{ccccccccccccccccc}
\hline Sub.nr & Sex & $\begin{array}{c}\text { Age } \\
\text { (yrs) }\end{array}$ & $\begin{array}{c}\text { Disease } \\
\text { dur. } \\
\text { (yrs) }\end{array}$ & $\begin{array}{c}\text { Tremor. } \\
\text { Dom. }\end{array}$ & & LEDD & $\begin{array}{c}\text { UPDRS } \\
\text { I }\end{array}$ & $\begin{array}{c}\text { UPDRS } \\
\text { II }\end{array}$ & $\begin{array}{c}\text { UPDRS } \\
\text { III }\end{array}$ & $\begin{array}{c}\text { UPDRS } \\
\text { IV }\end{array}$ & $\begin{array}{l}\text { \% OFF } \\
\text { HY }\end{array}$ & MMSE & PAS & BDI-II & PDQ-8 \\
\hline 1 & M & 57 & 8 & No & 600 & 1 & 15 & 49 & 6 & $26-50$ & 3 & 28 & 4 & 12 & 9 \\
2 & M & 53 & 7 & Yes & 800 & 4 & 12 & 21 & 10 & $1-25$ & 2.5 & 28 & 17 & 14 & 21 \\
3 & F & 77 & 3 & Yes & 800 & 6 & 17 & 28 & 8 & $26-50$ & 3 & 27 & 24 & 16 & 11 \\
4 & F & 62 & 6 & Yes & 712.5 & 5 & 11 & 34 & 4 & $26-50$ & 3 & 29 & 11 & 15 & 14 \\
5 & F & 61 & 5 & No & 690 & 3 & 9 & 8 & 7 & $1-25$ & 1.5 & 29 & 19 & 12 & 10 \\
6 & M & 73 & 11 & Yes & 1000 & 3 & 10 & 22 & 4 & $26-50$ & 1.5 & 30 & 3 & 1 & 5 \\
7 & M & 65 & 6 & Yes & 1100 & 1 & 14 & 22 & 9 & $1-25$ & 2,5 & 30 & 19 & 11 & 12 \\
8 & M & 63 & 8 & No & 1306 & 0 & 15 & 15 & 7 & $26-50$ & 2 & 29 & 10 & 1 & 7 \\
9 & M & 50 & 5 & No & 557 & 4 & 8 & 25 & 6 & $1-25$ & 2 & 30 & 10 & 8 & 9 \\
10 & F & 50 & 11 & Yes & 835 & 2 & 12 & 32 & 9 & $1-25$ & 2 & 30 & 20 & 16 & 13 \\
11 & M & 64 & 10 & No & 1242 & 2 & 8 & 16 & 10 & $1-25$ & 2 & 29 & 9 & 3 & 10 \\
\hline
\end{tabular}

Sub.nr $=$ Subject $\mathrm{nr}$, Disease dur. $=$ disease duration, tremor dom. $=$ tremor-dominant PD, LEDD = levodopa equivalent daily dose, UPDRS $=$ Unified

Parkinson Disease Rating Scale, \% OFF = UPDRS IV question 39, HY = Hoehn and Yahr stage, MMSE = Mini Mental State Examination, PAS = Parkinson Anxiety Scale, BDI-II = Beck Depression Inventory II, PDQ-8 = Parkinson's Disease Questionnaire 8.

Table 2. Descriptive statistics of ESM variables used in the analysis

\begin{tabular}{lccccc}
\hline & Mean & Median & SD & Variance & Range \\
\hline Positive affect & 4.55 & 5 & 1.40 & 1.97 & $1-7$ \\
Negative affect & 3.33 & 1.17 & .55 & .31 & $1-5$ \\
Tremor & 1.91 & 1 & 1.24 & 1.53 & $1-6$ \\
Rigidity & 1.59 & 2 & 1.23 & 2.12 & $1-7$ \\
Balance Problems & 2.49 & 1 & 1.46 & 1.50 & $1-7$ \\
Walking problems & 2.75 & 2 & 1.93 & 3.74 & $1-7$ \\
Dyskinesia & 1.93 & 1 & 1.30 & 1.68 & $1-7$ \\
\hline
\end{tabular}


structure. Next, the regression coefficient obtained from the real multilevel regression analysis was placed on that normal distribution to obtain p-values (i.e., the proportion of times that the coefficient in the permuted data was as large as or larger than the observed coefficient; multiplied by two to obtain a two-sided $p$-value $)^{27}$. Second, associations between the independent variables and the severity of motor symptoms independent of ON/OFF state were analysed in the full data set by multilevel linear regression analyses, including all independent variables in one model. Third, in order to assess the usability of ESM on an individual level, linear regression analyses were performed in data from individual patients using the same variables included in the full dataset. Moreover, graphs were generated to illustrate individual moment-to-moment fluctuations in mood and motor symptoms and ON/OFF state.

\section{RESULTS}

\section{Participants and descriptive statistics}

One participant was excluded from the analysis because of a response rate of $26 \%$, which is below the generally accepted minimum response rate of $33 \%$ for ESM data sampling ${ }^{14}$. Among the remaining 11 participants a total of 412 questionnaires were completed, corresponding to a mean response rate of $74.9 \%$. Between individual patients the response rate varied from $42 \%$ to $94 \%$ (supplementary table 2 ). The demographic and disease-related characteristics of the participants are summarised in table 1. The study population consisted of four females and seven males. Mean age was 62.3 years $(S D=7.8)$; mean disease duration was 7 years $(S D=2.6)$, and mean UPDRS part III score was $24.7(\mathrm{SD}=11.0)$. The median Hoehn and Yahr stage was 2 (range 1.5 to 3$)^{20}$. A total of 4 patients were on a stable dose of dopamine agonists during the course of the study (either ropinirole or pramipexole), 3 patients used levodopa/benserazide, 7 patients used levodopa/carbidopa, 1 patient used levodopa/carbidopa/entecapone and 4 patients used amantadine. On the PAS, five subjects (participants 2, 3, 5, 7 and 10) scored above the cut-off point of 14, indicating clinically relevant anxiety ${ }^{22}$. On the BDI-II, four subjects (participants 2, 3, 4 and 10) scored above the cut-off point of 14 for mild depression ${ }^{23}$. Mean scores for affective state and motor symptoms are shown in table 2 . Of the 412 completed questionnaires, 111 were completed in 'OFF' state (27\%) and 301 in 'ON' state (71\%). Even though motor fluctuations were present, one patient did not report any 'OFF' states. The percentage 'ON' varied from $46.7 \%$ to $100 \%$ in individual subjects. 


\section{Group level analyses}

\section{Effect modification of ON/OFF state}

Dyskinesia was not analysed, since five patients did not suffer from dyskinesia. Interaction analyses showed a statistically significant interaction between ON/OFF state and being at home when analysing tremor $\left(X^{2}(1)=4.8, p=0.028\right)$ (supplementary table 3). However, following permutation analysis, the association was no longer statistically significant (supplementary table 4). There were no other significant interaction effects.

Associations between affective state, contextual factors and motor symptom severity Multilevel linear regression analyses showed statistically significant negative associations between positive affect and all motor symptoms: higher scores on positive affect were associated with lower self-perceived severity of tremor, rigidity, balance problems and walking problems. In addition, being at home was associated with increased balance problems and rigidity, while being alone was associated with reduced rigidity (table 3 ).

Table 3. Associations between motor symptoms, mood states and contextual factors (independent of ON/OFF state)

\begin{tabular}{|c|c|c|c|c|c|c|c|c|}
\hline & \multicolumn{4}{|c|}{ Tremor } & \multicolumn{4}{|c|}{ Rigidity } \\
\hline & B & SE & $\mathbf{p}$ & $95 \% \mathrm{Cl}$ & B & SE & $\mathbf{p}$ & $95 \% \mathrm{Cl}$ \\
\hline PA & -0.16 & 0.06 & 0.007 & $-0.28--0.05$ & -0.26 & 0.07 & 0.000 & $-0.39--0.12$ \\
\hline NA & 0.12 & 0.12 & 0.29 & $-0.11-0.35$ & 0.28 & 0.14 & $0.041^{a}$ & $0.01-0.55$ \\
\hline At home & 0.17 & 0.13 & 0.18 & $-0.08-0.43$ & 0.41 & 0.15 & 0.008 & $0.10-0.71$ \\
\hline \multirow{3}{*}{$\begin{array}{l}\text { Being } \\
\text { alone }\end{array}$} & -0.14 & 0.12 & 0.21 & $-0.37-0.09$ & -0.42 & 0.14 & 0.002 & $-0.69--0.02$ \\
\hline & \multicolumn{4}{|c|}{ Walking problems } & \multicolumn{4}{|c|}{ Balance problems $^{a}$} \\
\hline & B & SE & $\mathbf{p}$ & $95 \% \mathrm{Cl}$ & B & SE & $\mathbf{p}$ & $95 \% \mathrm{Cl}$ \\
\hline PA & -0.23 & 0.07 & 0.001 & $-0.16--0.01$ & -0.09 & 0.04 & 0.032 & $-0.16--0.01$ \\
\hline NA & 0.18 & 0.13 & 0.17 & $-0.08-0.43$ & 0.06 & 0.08 & 0.42 & $-0.08-0.21$ \\
\hline At home & 0.23 & 0.14 & 0.11 & $-0.53-0.51$ & 0.22 & 0.08 & 0.010 & $0.05-0.51$ \\
\hline $\begin{array}{l}\text { Being } \\
\text { alone }\end{array}$ & -0.21 & 0.13 & 0.10 & $-0.46-0.04$ & -0.14 & 0.08 & 0.06 & $-0.29-0.01$ \\
\hline
\end{tabular}




\section{DISCUSSION}

The present study aimed to study if routinely collected ESM data over a brief period of time can be used to study associations between affective state, contextual factors, motor symptoms and motor state (ON/OFF) in PD patients who suffer from motor fluctuations on a group and individual level. Revealing population based patterns may lead to more general treatment recommendations, while individual analyses can be used to establish a personalised treatment plan ${ }^{14}$. Ideally, assessments are kept as brief as possible, in order to reduce the burden and improve compliance rates of patients ${ }^{14}$.

On the group level, ESM collected over a period of five consecutive days was able to identify clear associations between affective state, contextual factors and motor symptoms. Higher scores on positive affect were associated with lower self-perceived severity of tremor, rigidity, balance problems and walking problems. Being at home was associated with more balance problems and rigidity in general. The observed associations were independent of whether a patient was in an 'ON' or 'OFF' state.

When analysing data of individual patients, only a few subjects showed statistically significant associations between affective state, contextual factors and motor symptoms, which were mostly, but not always, in the same direction as the findings on the group level.

\section{Group level}

The finding that ON/OFF state was not a modifier in the associations between affective state and motor symptoms was surprising. As mood symptoms are mostly related to OFF states ${ }^{9}$, the relationship between positive or negative feelings and the severity of motor symptoms might be different when a patient is OFF compared to ON, e.g. there might be increased symptom focus in OFF state when experiencing negative emotions, or an increased symptom focus when OFF may lead to a stronger response in negative emotions. However, OFF states might have been underrepresented in the present study, leading to lack of power (see methodological issues below). As there are only few reports examining motor fluctuations using ESM data in PD patients, it is hard to compare the present findings with results from other studies. A recent study by Fernie et al. (2019) examined emotional distress and motor states in PD patients using ESM 
data and found that episodic distress is greater during OFF states compared to ON states, but worse during OFF-coming-ON compared to ON-wearing-OFF transitional states $^{28}$. Unfortunately, the present study could not identify these transitional states. Moreover, the study by Fernie et al. (2019) specifically focused on emotional distress and their questionnaire did not include different motor symptoms or any items reflecting positive emotions ${ }^{28}$.

Interestingly, we observed that positive affect was associated with reduced selfperceived symptom severity for all motor symptoms, while negative affect was not associated with any of the motor symptoms. Since the present data are crosssectional, causal inferences cannot be made. Therefore, we do not know whether a better mood results in reduced self-perceived severity of motor symptoms, or whether reduced self-perceived severity of motor symptoms results in better mood. Nonetheless, it seems that the relationship between motor symptoms and positive affect is more significant in PD, compared to the relationship between negative affect and motor symptoms. This finding is in line with results from the network analysis we recently performed on ESM data from one PD patient which aimed to identify longitudinal associations between motor symptoms and mood states ${ }^{17}$. While feeling 'down' did not influence motor symptom severity, 'cheerfulness' was significantly associated with better scores for tremor and rigidity in this $\mathrm{N}=1$ study ${ }^{17}$. The associations between mood and motor states were independent of whether a patient subjectively perceived to be in an ON or OFF state. The dosage and timing of medication may have served as a modifier between the observed associations. Previous studies have demonstrated mood changes related to L-dopa infusion in a dose-dependent fashion ${ }^{29-31}$. For future research it would be of clinical relevance to include questions regarding timing and dosage of medication. Moreover, most studies on the effects of affective state on motor symptoms in PD have focused on negative mood states, such as depression, anxiety and apathy, ignoring positive affect ${ }^{9}$. In the field of psychiatry, there is increasing evidence that the impact of interventions, both pharmacological and non-pharmacological, is mediated more by enhancing positive emotions, than by reducing negative emotions ${ }^{32,33}$. It is, therefore, important to include positive affect in the design of future studies. Moreover, enhancing the frequency and persistence of positive affect in daily life might be a promising adjunctive nonpharmacological target for reducing the burden of motor fluctuations. 
Analyses of the associations between contextual factors on the one hand and selfperceived motor symptoms on the other hand showed that, overall, subjects perceived more rigidity and balance problems when at home. Possibly, patients have a tendency to stay at home when experiencing balance problems. Balance problems are among the most distressing symptoms related to $\mathrm{PD}^{34}$, as they are often accompanied by fear of falling ${ }^{35}$, thereby limiting a patient's mobility and independence. Alternatively, being at home could also contribute to increased symptom focus or an increased chance of being confronted with balance problems due to the presence of more obstacles in smaller rooms. The negative association between rigidity and being alone is more difficult to explain, as there were no other statistically significant associations between being alone and motor symptoms. In general, context-specific associations based on analyses of a group of patients are difficult to interpret without patient-feedback. Context is an important consideration for many symptoms and the purpose of including contextual factors in ESM sampling strategies is to reveal complex environmental dynamics that can potentially help individuals to optimise environmental interactions and benefit coping strategies ${ }^{32}$.

\section{Individual level}

Overall, we found less clear associations when analysing routinely collected ESM data in individual patients. While most associations between being at home and balance problems in individual patients were also positive but not significant, to our surprise, the only patient with a statistically significant association was in opposite direction compared to the findings on a group level. Regarding positive and negative affect, most associations were in the same direction compared to the group analyses, but not all. One could assume that those subjects that suffered from clinically relevant anxiety and/or depression at baseline would show more significant associations between negative affect and motor symptoms. Except for subject 10, who showed statistically significant associations for both positive and negative affect, there was only one other patient with baseline anxiety in whom negative affect was associated with increased balance problems. We think the most likely explanation for the failure to identify significant associations between symptoms in individual patients is lack of power.

On the other hand, the graphs were able to display moment-to-moment fluctuations in mood and motor symptoms. For clinical practice, this might provide the clinician 
sufficient information that was otherwise not available via retrospective interviews or routine cross-sectional assessments in which a single assessment of symptom severity is considered representative for a longer time frame. Graphical representations of symptoms might also give the patient more insight into particular circumstances in which fluctuations occur or are perceived as more disabling, especially when fluctuations have become unpredictable. Apart from using ESM data to visualise and monitor symptom change following medication adjustments, it can also be used to guide collaborate decision making between the patient and clinician, e.g. determining which information is most important to monitor by ESM or to support treatment target selection ${ }^{14,32}$.

\section{Methodological issues}

This study has several limitations. First, the sample size was small. As ESM generates a large number of data due to intensive data collection, we were able to reveal group level associations. However, for the individual analyses, five days of ESM data collection is probably too short to obtain sufficient power. In order to be applicable in routine patient care, the sensitivity of the method needs to be increased. This may be done by collecting data over a longer period of time as previously shown in our $\mathrm{N}=1$ study, by increasing the compliance or by making the answers more sensitive to change (e.g. by increasing the number of potential scores on the Likert item or by using visual analogue scale scores). Similarly, the finding that ON/OFF state was not a modifier in the studied associations might also be due to lack of power, as only $27 \%$ of completed questionnaires were in OFF state. While highly fluctuating states might be under-sampled with an ESM sampling frequency of 10 times per day, slowly fluctuating states might be over-sampled ${ }^{14}$. It is possible that with the current ESM protocol motor fluctuations were not adequately represented. For that reason, we did not include ON/OFF state in the analyses of individual subjects. In order to adequately sample ON/OFF fluctuations, the interval between assessments should be adapted to fit the frequency and duration of the OFF periods. An alternative approach would be to allow the patient to complete 'patient-generated beep assessments' during OFF periods, or to program the assessments at fixed times. However, with the latter method one may run the risk of missing the fluctuating nature of symptoms. Third, we did not include intermediate or transitional motor states, such as ON-wearing-OFF or OFF-coming$\mathrm{ON}$, which might reveal different and potential relevant associations between affective 
states and motor symptoms. In addition, ON/OFF state was defined subjectively by each individual subject. The use of wearable devices to objectify motor fluctuations may be a valuable addition in this respect. Recently, a study showed that combining wearable sensors with ESM data is feasible ${ }^{36}$. Fourth, the current study had a crosssectional approach, which makes it impossible to study assess temporal or causal associations. Studying temporal associations requires much longer periods of data collection, which may be too long to be implemented in routine patient care. In the context of an $\mathrm{N}=1$ study we collected ESM data for a longer period of time - one monthin a PD patient. Although we were able to reveal longitudinal associations between affective state and motor symptoms in this patient, his response rate declined more than $50 \%$ over the course of 30 days, which may reflect the burden that patients experience when collecting data over a longer period of time ${ }^{17}$. Fifth, four patients used a (stable) dose of dopamine agonists during the study, which may be a confounder when measuring mood states. With the analysis performed in the present study it is not possible to correct for such confounders. For future research and for clinical practice it would be useful to record the time of medication intake, as well as missed or delayed intakes, to monitor the effect of dopaminergic treatment on motor symptoms or mood fluctuations. Sixth, we could not include random effects for the slopes of all predictor variables included in the models of the multilevel linear regression analysis; i.e. both the interaction analyses and the main effects, which might lead to an overestimation of $p$-values. Moreover, the assumption of normality of residuals was not met for all variables (e.g. for balance problems). To obtain valid and interpretable results, we performed permutation testing. As these sensitivity analyses revealed similar results in all but two cases (the interaction between ON/OFF state and being at home when analysing tremor, and the association between rigidity and negative affect), we adopted the results from the multilevel linear regression analyses and did not perform further permutation testing on data of individual subjects. Seventh, in three subjects the contextual factor 'being alone' was removed from the linear regression model because those subjects were always alone when at home and in company when not at home. Thus, the regression coefficients presented in supplementary table 6 were in fact the association between being home alone and the respective outcomes. Finally, the results are based on a priori selected variables that aimed to represent positive and negative mood states, as well as contextual factors and should therefore be considered with caution. Selecting different (combinations of) items might lead to 
different results. For clinical practice, items are ideally selected based on the specific situation of the patient in a collaborative process between clinician and patient.

\section{CONCLUSION}

The ultimate goal is to use ESM in a non-burdening way in routine patient care as a tool to facilitate patient self-monitoring and as a guidance for clinicians to personalise treatment. Whereas in this study, ESM collected over a period of five days appeared sensitive enough to reveal associations between motor symptoms, mood states and contextual factors on a group level, this approach was not powerful enough to reveal an adequate number of relevant association on an individual level. This implies that the method must be made more sensitive for application in routine clinical practice. Since ESM will probably play a more important role in future studies as well as in clinical practice, we argue that there is an urgent need of harmonisation, validation and optimisation of this technique for use in PD patients. 


\section{REFERENCES}

1. LeWitt PA. Levodopa therapy for Parkinson's disease: Pharmacokinetics and pharmacodynamics. Mov Disord 2015;30(1):64-72.

2. Stocchi F, Antonini A, Barone P, et al. Early DEtection of wEaring off in Parkinson disease: the DEEP study. Parkinsonism Relat Disord 2014;20(2):204-211.

3. Ahlskog JE, Muenter MD. Frequency of levodopa-related dyskinesias and motor fluctuations as estimated from the cumulative literature. Mov Disord 2001;16(3):448-458.

4. (WHO) WHO. Neurological disorders - public health challenges. Geneva, Switzerland: World Health Organization, 2006.

5. Memedi M, Sadikov A, Groznik V, et al. Automatic Spiral Analysis for Objective Assessment of Motor Symptoms in Parkinson's Disease. Sensors (Basel) 2015;15(9):23727-23744.

6. Hechtner MC, Vogt T, Zollner Y, et al. Quality of life in Parkinson's disease patients with motor fluctuations and dyskinesias in five European countries. Parkinsonism Relat Disord 2014;20(9):969-974.

7. Storch A, Schneider CB, Wolz M, et al. Nonmotor fluctuations in Parkinson disease: severity and correlation with motor complications. Neurology 2013;80(9):800-809.

8. Witjas T, Kaphan E, Azulay JP, et al. Nonmotor fluctuations in Parkinson's disease: frequent and disabling. Neurology 2002;59(3):408-413.

9. van der Velden RMJ, Broen MPG, Kuijf ML, Leentjens AFG. Frequency of mood and anxiety fluctuations in Parkinson's disease patients with motor fluctuations: A systematic review. Mov Disord 2018;33(10):1521-1527.

10. Bradburn NM, Rips LJ, Shevell SK. Answering autobiographical questions: the impact of memory and inference on surveys. Science 1987;236(4798):157-161.

11. Broen MP, Marsman VA, Kuijf ML, Van Oostenbrugge RJ, van Os J, Leentjens AF. Unraveling the Relationship between Motor Symptoms, Affective States and Contextual Factors in Parkinson's Disease: A Feasibility Study of the Experience Sampling Method. PLoS One 2016;11(3):e0151195.

12. Mujagic Z, Leue C, Vork L, et al. The Experience Sampling Method--a new digital tool for momentary symptom assessment in IBS: an exploratory study. Neurogastroenterol Motil 2015;27(9):1295-1302.

13. Palmier-Claus JE, Myin-Germeys I, Barkus E, et al. Experience sampling research in individuals with mental illness: reflections and guidance. Acta Psychiatr Scand 2011;123(1):12-20.

14. Verhagen SJW, Berben JA, Leue C, et al. Demonstrating the reliability of transdiagnostic mHealth Routine Outcome Monitoring in mental health services using experience sampling technology. PLoS One 2017;12(10):e0186294.

15. Csikszentmihalyi M, Larson R. Validity and reliability of the Experience-Sampling Method. J Nerv Ment Dis 1987;175(9):526-536.

16. Myin-Germeys I, Oorschot M, Collip D, Lataster J, Delespaul P, van Os J. Experience sampling research in psychopathology: opening the black box of daily life. Psychol Med 2009;39(9):1533-1547.

17. van der Velden RMJ, Mulders AEP, Drukker M, Kuijf ML, Leentjens AFG. Network analysis of symptoms in a Parkinson patient using experience sampling data: $\mathrm{An} n=1$ study. Mov Disord 2018;33(12):1938-1944.

18. Hughes AJ, Daniel SE, Kilford L, Lees AJ. Accuracy of clinical diagnosis of idiopathic Parkinson's disease: a clinico-pathological study of 100 cases. J Neurol Neurosurg Psychiatry 1992;55(3):181-184.

19. Folstein MF, Folstein SE, McHugh PR. "Mini-mental state". A practical method for grading the cognitive state of patients for the clinician. J Psychiatr Res 1975;12(3):189-198.

20. Hoehn MM, Yahr MD. Parkinsonism: onset, progression and mortality. Neurology 1967;17(5):427-442.

21. Movement Disorder Society Task Force on Rating Scales for Parkinson's Disease. The Unified Parkinson's Disease Rating Scale (UPDRS): status and recommendations. Mov Disord 2003;18(7):738750. 
22. Leentjens AF, Dujardin K, Pontone GM, Starkstein SE, Weintraub D, Martinez-Martin P. The Parkinson Anxiety Scale (PAS): development and validation of a new anxiety scale. Mov Disord 2014;29(8):1035-1043.

23. Beck AT, Steer RA, Ball R, Ranieri W. Comparison of Beck Depression Inventories -IA and -II in psychiatric outpatients. J Pers Assess 1996;67(3):588-597.

24. Jenkinson C, Fitzpatrick R, Peto V, Greenhall R, Hyman N. The PDQ-8: development and validation of a short-form Parkinson's disease questionnaire. Psychology and Health 1997;12(6):805814.

25. Corp I. IBM SPSS Statistics for Windows. Version 24.0. 24.0 ed. Armonk, NY: IBM Corp; 2016. 26. StataCorp. Stata Statistical Software: Release 13. College Station, TX: StataCorp LP 2013.

27. Hasmi L, Drukker M, Guloksuz S, et al. Genetic and Environmental Influences on the Affective Regulation Network: A Prospective Experience Sampling Analysis. Front Psychiatry 2018;9:602.

28. Fernie BA, Spada MM, Brown RG. Motor fluctuations and psychological distress in Parkinson's disease. Health Psychol 2019;38(6):518-526.

29. Cantello R, Gilli M, Riccio A, Bergamasco B. Mood changes associated with "end-of-dose deterioration" in Parkinson's disease: a controlled study. J Neurol Neurosurg Psychiatry 1986;49(10):1182-1190.

30. Maricle RA, Nutt JG, Valentine RJ, Carter JH. Dose-response relationship of levodopa with mood and anxiety in fluctuating Parkinson's disease: a double-blind, placebo-controlled study. Neurology 1995;45(9):1757-1760.

31. Maricle RA, Valentine RJ, Carter J, Nutt JG. Mood response to levodopa infusion in early Parkinson's disease. Neurology 1998;50(6):1890-1892.

32. van Os J, Verhagen $\mathrm{S}$, Marsman $\mathrm{A}$, et al. The experience sampling method as an mHealth tool to support self-monitoring, self-insight, and personalized health care in clinical practice. Depress Anxiety 2017;34(6):481-493.

33. Hartmann JA, Wichers M, Menne-Lothmann C, et al. Experience Sampling-Based Personalized Feedback and Positive Affect: A Randomized Controlled Trial in Depressed Patients. Plos One 2015;10(6).

34. Backer JH. The symptom experience of patients with Parkinson's disease. J Neurosci Nurs 2006;38(1):51-57.

35. Franchignoni F, Martignoni E, Ferriero G, Pasetti C. Balance and fear of falling in Parkinson's disease. Parkinsonism Relat Disord 2005;11(7):427-433.

36. Heijmans M, Habets JGV, Herff C, et al. Monitoring Parkinson's disease symptoms during daily life: a feasibility study. npj Parkinson's Disease 2019;5(1):21. 


\section{Supplementary materials}

Supplementary Table 1. ESM questionnaire

\begin{tabular}{|c|c|c|c|}
\hline Domain & Item & Description & Score \\
\hline Mood & $\begin{array}{l}1 \\
2 \\
3 \\
4 \\
5 \\
6 \\
7 \\
8 \\
9 \\
10 \\
11 \\
12 \\
13\end{array}$ & $\begin{array}{l}\text { I feel happy } \\
\text { I feel insecure } \\
\text { I feel relaxed } \\
\text { I feel irritated } \\
\text { I feel satisfied } \\
\text { I feel lonely } \\
\text { I feel afraid } \\
\text { I feel down } \\
\text { I feel guilty } \\
\text { I rack my brain } \\
\text { I feel suspicious } \\
\text { I feel threatened } \\
\text { In general, I feel well }\end{array}$ & $\begin{array}{l}1-7(1=\text { not, } 4=\text { moderate, } 7=\text { very }) \\
1-7 \\
1-7 \\
1-7 \\
1-7 \\
1-7 \\
1-7 \\
1-7 \\
1-7 \\
1-7 \\
1-7 \\
1-7 \\
1-7\end{array}$ \\
\hline Parkinson & $\begin{array}{l}14 \\
15 \\
16 \\
17 \\
18 \\
19 \\
20\end{array}$ & $\begin{array}{l}\text { Currently I am: } \\
\text { I experience tremor } \\
\text { I experience rigidity } \\
\text { Walking is difficult } \\
\text { I experience balance problems } \\
\text { I experience dyskinesia } \\
\text { Personal complaints: } \\
\end{array}$ & $\begin{array}{l}0=" O F F ", 1=" O N " \\
1-7 \\
1-7 \\
1-7 \\
1-7 \\
1-7 \\
\text { Open question }\end{array}$ \\
\hline Context & $\begin{array}{l}22 \\
23 \\
\\
24 \\
25\end{array}$ & $\begin{array}{l}\text { Currently I am: } \\
\text { I rather do something else } \\
\text { Where am I? } \\
\text { With who am I? } \\
\text { I find this pleasant }\end{array}$ & $\begin{array}{l}\text { Resting/ working/ housework/ hygiene/ } \\
\text { eating, drinking/ relaxing/ } \\
\text { conversating/ other } \\
1-7 \\
\text { Home/ work/ friends place/ public } \\
\text { place/ on the go } \\
\text { Nobody/ partner/ family/ friends/ } \\
\text { collegues/ strangers } \\
1-7\end{array}$ \\
\hline Somatic & $\begin{array}{l}26 \\
27 \\
28 \\
29 \\
30 \\
31\end{array}$ & $\begin{array}{l}\text { I am hungry } \\
\text { Since the last beep I used: } \\
\text { I am tired } \\
\text { I am not feeling well } \\
\text { I have pain } \\
\text { I experience: }\end{array}$ & $\begin{array}{l}1-7 \\
\text { Nothing /caffeine/ nicotine/ alcohol/ } \\
\text { medication/ cannabis/ food/ other } \\
1-7 \\
1-7 \\
1-7 \\
\text { Headache/ abdominal pain/ breathing } \\
\text { difficulties/ muscle ache/ obstipation/ } \\
\text { tinnitus/ dizziness/ dyspnea/ } \\
\text { palpitations/ incontinence }\end{array}$ \\
\hline Events & $\begin{array}{l}32 \\
33 \\
34 \\
\\
35 \\
36 \\
37 \\
\end{array}$ & $\begin{array}{l}\text { The most important event since the } \\
\text { last beep was: } \\
\text { The event was: } \\
\text { The event was: } \\
\text { The event was under my control } \\
\text { I expected this event } \\
\text { This beep was unpleasant }\end{array}$ & $\begin{array}{l}\text { Open question } \\
-3-3(-3=\text { very displeasing, } 0= \\
\text { neutral, } 3=\text { very pleasant }) \\
-3-3(-3=\text { not important, } 0=\text { neutral, } 3 \\
=\text { very important }) \\
1-7 \\
1-7 \\
1-7\end{array}$ \\
\hline
\end{tabular}


Supplementary Table 2: Overview of response rates

\begin{tabular}{lcccc}
\hline Subject & $\begin{array}{c}\text { Completed } \\
\text { questionnaires (nr) }\end{array}$ & $\begin{array}{c}\text { Response } \\
\text { percentage (\%) }\end{array}$ & ON / OFF (nr) & $\begin{array}{c}\text { Percentage ON } \\
(\%)\end{array}$ \\
\hline 1 & 21 & 42 & $19 / 2$ & 90.5 \\
2 & 26 & 52 & $23 / 3$ & 88.5 \\
3 & 42 & 84 & $42 / 0$ & 100 \\
4 & 35 & 70 & $18 / 17$ & 51.4 \\
5 & 46 & 92 & $28 / 18$ & 60.9 \\
6 & 47 & 94 & $27 / 20$ & 57.4 \\
7 & 30 & 60 & $14 / 16$ & 46.7 \\
8 & 39 & 78 & $26 / 13$ & 66.7 \\
9 & 46 & 92 & $42 / 4$ & 91.3 \\
10 & 45 & 90 & $36 / 9$ & 80 \\
11 & 35 & 70 & $26 / 9$ & 74.3 \\
\hline Total & 412 & 74.9 & $301 / 111$ & 73.1 \\
\hline
\end{tabular}

Supplementary Table 3. Interaction effects ON/OFF state

\begin{tabular}{|c|c|c|c|c|c|c|c|c|}
\hline & \multicolumn{4}{|c|}{ Tremor } & \multicolumn{4}{|c|}{ Rigidity } \\
\hline & B & SE & $\mathbf{p}$ & $95 \% \mathrm{Cl}$ & B & SE & $p$ & $95 \% \mathrm{Cl}$ \\
\hline PA & 0.04 & 0.07 & 0.59 & $-0.11-0.18$ & -0.08 & 0.09 & 0.39 & $-0.10-0.02$ \\
\hline NA & 0.08 & 0.19 & 0.69 & $-0.29-0.45$ & -0.26 & 0.23 & 0.25 & $-0.72-0.19$ \\
\hline At home & -0.54 & 0.25 & 0.028 & $-.102-.057$ & -0.35 & 0.30 & 0.25 & $-0.25-0.94$ \\
\hline \multirow{3}{*}{ Being alone } & -0.24 & 0.20 & 0.24 & $-0.63-0.16$ & 0.33 & 0.25 & 0.19 & $-0.16-0.81$ \\
\hline & \multicolumn{4}{|c|}{ Walking problems } & \multicolumn{4}{|c|}{ Balance problems $^{a}$} \\
\hline & B & SE & $p$ & $95 \% \mathrm{Cl}$ & B & SE & $p$ & $95 \% \mathrm{Cl}$ \\
\hline PA & -0.15 & 0.09 & 0.07 & $-0.32-0.02$ & -0.05 & 0.05 & 0.32 & $-0.16-0.01$ \\
\hline NA & -0.09 & 0.22 & 0.70 & $-0.52-0.35$ & 0.16 & 0.14 & 0.23 & $-0.10-0.43$ \\
\hline At home & 0.31 & 0.29 & 0.29 & $-0.26-0.05$ & 0.25 & 0.18 & 0.15 & $-0.09-0.60$ \\
\hline Being alone & -0.04 & 0.24 & 0.87 & $-0.50-0.42$ & 0.10 & 0.15 & 0.27 & $-0.12-0.43$ \\
\hline
\end{tabular}

Supplementary Table 4. P-values original analyses and permutation analyses

\begin{tabular}{|c|c|c|c|c|c|c|c|c|c|c|}
\hline & \multicolumn{2}{|c|}{$\begin{array}{l}\text { Tremor - } \\
\text { ON/OFF }\end{array}$} & \multicolumn{2}{|c|}{ Tremor } & \multicolumn{2}{|c|}{ Rigidity } & \multicolumn{2}{|c|}{$\begin{array}{l}\text { Walking } \\
\text { problems }\end{array}$} & \multicolumn{2}{|c|}{$\begin{array}{l}\text { Balance } \\
\text { problems }\end{array}$} \\
\hline & $\mathbf{P}$ & P-PT & $\mathbf{P}$ & P- PT & $\mathbf{P}$ & P- PT & $\mathbf{P}$ & P-PT & $\mathbf{P}$ & P- PT \\
\hline PA & 0.59 & 0.99 & 0.007 & 0.002 & $<0.001$ & 0.006 & 0.001 & $<0.001$ & 0.032 & 0.039 \\
\hline NA & 0.69 & 0.54 & 0.29 & 0.21 & 0.041 & 0.076 & 0.17 & 0.19 & 0.42 & 0.37 \\
\hline $\begin{array}{l}\text { At } \\
\text { home }\end{array}$ & 0.028 & 0.99 & 0.13 & 0.14 & 0.008 & 0.001 & 0.11 & 0.11 & 0.010 & 0.013 \\
\hline $\begin{array}{l}\text { Being } \\
\text { alone }\end{array}$ & 0.24 & 0.99 & 0.12 & 0.23 & 0.002 & $<0.001$ & 0.10 & 0.08 & 0.06 & 0.06 \\
\hline
\end{tabular}


Supplementary Table 5: Associations between motor symptoms and mood states in individual patients

\begin{tabular}{|c|c|c|c|c|c|c|c|c|c|}
\hline \multirow{3}{*}{$\begin{array}{l}\mathrm{Pt} \\
+\mathrm{N}\end{array}$} & \multirow[b]{3}{*}{ Symptom } & \multicolumn{8}{|c|}{ Mood } \\
\hline & & \multicolumn{4}{|c|}{ Positive affect } & \multicolumn{4}{|c|}{ Negative affect } \\
\hline & & B & SE & $\mathbf{p}$ & $95 \% \mathrm{CI}$ & B & SE & $\mathbf{p}$ & $95 \% \mathrm{Cl}$ \\
\hline & Tremor & .102 & .403 & 0.804 & $-.753-.957$ & -.224 & .733 & 0.764 & $-1.778-1.330$ \\
\hline 1 & Rigidity & -.473 & .505 & 0.363 & $-1.544-.598$ & 1.862 & .918 & 0.060 & $-.084-3.808$ \\
\hline \multirow[t]{3}{*}{$\mathrm{N}=21$} & Walking & -1.027 & .402 & 0.021 & -1.880--.174 & 1.070 & .731 & 0.163 & $-.481-2.620$ \\
\hline & Balance & -.431 & .322 & 0.199 & $-1.113-.251$ & .688 & .585 & 0.257 & $-.552-1.927$ \\
\hline & Tremor & -.138 & .448 & 0.762 & $-1.073-.797$ & -.425 & .617 & 0.498 & $-1.771-.861$ \\
\hline $2 \mathrm{ab}$ & Rigidity & .078 & .414 & 0.853 & $-.786-.942$ & -.196 & .570 & 0.735 & $-1.384-.993$ \\
\hline \multirow[t]{3}{*}{$N=26$} & Walking & -.181 & .162 & 0.277 & $-.518-.156$ & -.116 & .222 & 0.607 & $-.580-.347$ \\
\hline & Balance & -.095 & .229 & 0.681 & $-.574-.383$ & .0251 & .315 & 0.937 & $-.632-.683$ \\
\hline & Tremor & .025 & .092 & 0.079 & $-.161-.210$ & .152 & .203 & 0.457 & $-.258-.563$ \\
\hline $3^{a b}$ & Rigidity & .042 & .204 & 0.838 & $-.372-.456$ & .248 & .452 & 0.587 & $-.668-1.163$ \\
\hline \multirow[t]{3}{*}{$\mathrm{N}=42$} & Walking & .039 & .175 & 0.825 & $-.315-.393$ & .321 & .386 & 0.412 & $-.462-1.104$ \\
\hline & Balance & .073 & .086 & 0.401 & $-.101-.246$ & -.126 & .189 & 0.509 & $-.509-.257$ \\
\hline & Tremor & .568 & .315 & 0.083 & $-.078-1.213$ & .768 & .838 & 0.367 & $-.947-2.483$ \\
\hline $4^{b}$ & Rigidity & -.291 & .310 & 0.356 & -.925-.344 & -.014 & .824 & 0.986 & $-1.699-1.670$ \\
\hline \multirow[t]{3}{*}{$\mathrm{N}=35$} & Walking & .079 & .337 & 0.815 & $-.609-.768$ & .393 & .895 & 0.664 & $-1.436-2.223$ \\
\hline & Balance & .025 & .050 & 0.625 & $-.078-.127$ & -.035 & .133 & 0.794 & -.307-.237 \\
\hline & Tremor & -.085 & .042 & 0.050 & -.169--.001 & .056 & .052 & 0.284 & $-.049-.162$ \\
\hline $5^{a}$ & Rigidity & -.372 & .202 & 0.072 & $-.780-.035$ & -.263 & .251 & 0.300 & $-.771-.244$ \\
\hline \multirow[t]{2}{*}{$N=46$} & Walking & -.252 & .178 & 0.165 & $-.612-.108$ & -.29 & .222 & 0.200 & $-.737-.159$ \\
\hline & Balance $^{c}$ & . & . & . & . & . & . & . & . \\
\hline & Tremor & .105 & .409 & 0.798 & $-.721-.931$ & -2.06 & 1.43 & 0.159 & $-4.95-.837$ \\
\hline 6 & Rigidity & -.111 & .198 & 0.577 & $-.511-.286$ & -1.483 & .692 & 0.038 & $-2.882--.084$ \\
\hline \multirow[t]{3}{*}{$N=47$} & Walking & -.148 & .301 & 0.627 & $-.755-.460$ & .725 & 1.054 & 0.495 & $-1.403-2.855$ \\
\hline & Balance & .076 & .104 & 0.468 & -.133-.286 & -.244 & .364 & 0.506 & -.979-.490 \\
\hline & Tremor & -2.42 & .756 & 0.004 & $-3.977--.862$ & .083 & 1.38 & 0.952 & $-2.760-2.927$ \\
\hline $7^{a}$ & Rigidity & -.969 & .466 & 0.048 & $-1.928-.010$ & 1.72 & .850 & 0.054 & $-.030-3.472$ \\
\hline \multirow[t]{3}{*}{$N=30$} & Walking & -1.032 & .591 & 0.093 & $-2.250-.187$ & -.093 & .108 & 0.932 & $-2.317-2.131$ \\
\hline & Balance & -.070 & .217 & 0.750 & $-.516-.377$ & 1.190 & .396 & 0.006 & $.375-2.005$ \\
\hline & Tremor & -.001 & .053 & 0.980 & $-.109-.106$ & .109 & .613 & 0.860 & $-1.137-1.356$ \\
\hline 8 & Rigidity & -.679 & .425 & 0.120 & $-1.543-.186$ & -2.911 & 4.918 & 0.558 & $-12.91-1.094$ \\
\hline \multirow[t]{2}{*}{$N=39$} & Walking & -.702 & .427 & 0.110 & $-1.571-.167$ & -1.926 & 1.945 & 0.699 & $-11.986-8.134$ \\
\hline & Balance & -.636 & .310 & 0.049 & -1.268--.004 & 1.977 & 3.598 & 0.586 & $-5.343-9.296$ \\
\hline & Tremor & -.085 & .054 & 0.125 & $-.194-.025$ & -.010 & .085 & 0.908 & $-.182-.162$ \\
\hline 9 & Rigidity & -.694 & .206 & 0.002 & -1.110--.278 & .069 & .324 & 0.833 & $-.588-.725$ \\
\hline \multirow[t]{3}{*}{$N=46$} & Walking & -.417 & .143 & 0.006 & -.705-.128 & .093 & .225 & 0.683 & $-.363-.548$ \\
\hline & Balance & -.241 & .099 & 0.020 & $-.441--.040$ & -.016 & .157 & 0.919 & $-.332-.300$ \\
\hline & Tremor & -.104 & .246 & 0.000 & $-1.536--.541$ & 1.91 & .634 & 0.004 & $.632-3.195$ \\
\hline $10 \mathrm{ab}$ & Rigidity & -.369 & .131 & 0.008 & -.631--.103 & .751 & .336 & 0.031 & $.070-1.431$ \\
\hline \multirow[t]{3}{*}{$\mathrm{N}=45$} & Walking & -.235 & .100 & 0.025 & -.438--.032 & .612 & .259 & 0.023 & $.089-1.126$ \\
\hline & Balance & .058 & .091 & 0.529 & $-.126-.242$ & .624 & .234 & 0.011 & $.150-1.098$ \\
\hline & Tremor & -.237 & .179 & 0.196 & $-.602-.129$ & .256 & .337 & 0.452 & $-.432-.945$ \\
\hline 11 & Rigidity & -.236 & .313 & 0.457 & $-.876-.404$ & .959 & .590 & 0.115 & $-.247-.2 .163$ \\
\hline \multirow[t]{2}{*}{$N=35$} & Walking & -.157 & .304 & 0.610 & $-.778-.465$ & 1.101 & .572 & 0.064 & $-.070-.2 .271$ \\
\hline & Balance & .033 & .149 & 0.826 & $.-.272-.339$ & .476 & .281 & 0.101 & -.099-.1.052 \\
\hline
\end{tabular}

$\overline{\mathrm{N}}=$ number of completed questionnaires, Walking $=$ walking problems, balance $=$ balance problems. Each participant received 50 assessments over the course of 5 days. Bold= significant result $p<0.05$

a Subject suffered from clinically relevant anxiety as objectified by the Parkinson Anxiety Scale ${ }^{20}$ b Subject suffered from clinically relevant depression as objectified by the Beck Depression Inventory- $12^{21}$ c Subject did not experience any balance problems during ESM data collection (score $=1$ ) 
Supplementary Table 6: Associations between motor symptoms and contextual factors in individual patients

\begin{tabular}{|c|c|c|c|c|c|c|c|c|c|}
\hline \multirow{3}{*}{$\begin{array}{l}\mathrm{Pt} \\
+\mathrm{N}\end{array}$} & \multirow[b]{3}{*}{ Symptom } & \multicolumn{8}{|c|}{ Contextual factors } \\
\hline & & \multicolumn{4}{|c|}{ At home } & \multicolumn{4}{|c|}{ Being alone } \\
\hline & & B & SE & $\mathbf{p}$ & $95 \% \mathrm{Cl}$ & B & SE & $\mathbf{p}$ & $95 \% \mathrm{Cl}$ \\
\hline \multirow{4}{*}{$\begin{array}{c}1 \\
N=21\end{array}$} & Tremor & .617 & .596 & 0.316 & $-.647-1.881$ & . & $\cdot$ & . & \\
\hline & Rigidity & .392 & .746 & 0.607 & $-1.191-1.974$ & . & . & . & . \\
\hline & Walking & .868 & .595 & 0.164 & $-.393-2.129$ & . & . & . & . \\
\hline & Balance & .685 & .476 & 0.169 & $-.323-1.693$ & . & . & . & . \\
\hline \multirow{4}{*}{$\begin{array}{c}2{ }^{a b} \\
\mathbf{N}=\mathbf{2 6}\end{array}$} & Tremor & .046 & .656 & 0.945 & $-1.322-1.414$ & .449 & .696 & 0.527 & $-1.004-1.901$ \\
\hline & Rigidity & 1.155 & .606 & 0.071 & $-.10-2.419$ & .242 & .643 & 0.711 & $-1.100-1.584$ \\
\hline & Walking & .017 & .236 & 0.944 & $-.476-.509$ & -.133 & .251 & 0.602 & $-.656-.390$ \\
\hline & Balance & .680 & .335 & 0.056 & $-.020-1.379$ & .054 & .356 & 0.882 & $-.689-.796$ \\
\hline \multirow{4}{*}{$\begin{array}{c}3^{a b} \\
N=42\end{array}$} & Tremor & .680 & .395 & 0.093 & $-.119-1.480$ & $\cdot$ &. & & \\
\hline & Rigidity & .093 & .880 & 0.916 & $-1.689-1.876$ & . & . & . & \\
\hline & Walking & -.017 & .752 & 0.982 & $-1.541-1.508$ & . & . & . & \\
\hline & Balance & .116 & .368 & 0.755 & $-.631-.862$ & . & . & . & \\
\hline \multirow{4}{*}{$\begin{array}{c}4^{b} \\
N=35\end{array}$} & Tremor & 1.114 & .921 & 0.236 & $-.769-2.998$ & -.780 & .402 & 0.062 & $-1.603-.043$ \\
\hline & Rigidity & .462 & .905 & 0.613 & $-1.388-2.313$ & -.607 & .395 & 0.135 & $-1.415-.201$ \\
\hline & Walking & -.405 & .983 & 0.683 & $-2.415-1.604$ & .423 & .429 & 0.333 & $-.522-1.301$ \\
\hline & Balance & .094 & .146 & 0.524 & -.204-.393 & -.063 & .064 & 0.332 & -.193-.068 \\
\hline \multirow{4}{*}{$\begin{array}{c}5^{a} \\
N=46\end{array}$} & Tremor & .109 & .074 & 0.148 & $-.040-.259$ & -.105 & .079 & 0.189 & $-.264-.054$ \\
\hline & Rigidity & -.172 & .357 & 0.633 & $-.893-.549$ & -.066 & .380 & 0.863 & $-.834-.702$ \\
\hline & Walking & -.206 & .315 & 0.516 & $-.844-.431$ & -.104 & .336 & 0.759 & $-.783-.575$ \\
\hline & Balance & . & . & . & . & . & . & . & \\
\hline \multirow{4}{*}{$\begin{array}{c}6 \\
N=47\end{array}$} & Tremor & -.465 & .433 & 0.289 & $-1.340-.410$ & -.203 & .316 & 0.525 & $-.842-.436$ \\
\hline & Rigidity & -.406 & .209 & 0.059 & -.829-.017 & -.218 & .153 & 0.162 & $-.527-.091$ \\
\hline & Walking & -.052 & .319 & 0.870 & $-.696-.591$ & .022 & .233 & 0.924 & $-.448-.492$ \\
\hline & Balance & .072 & .110 & 0.516 & $-.150-.294$ & .012 & .080 & 0.878 & $-.150-.175$ \\
\hline \multirow{4}{*}{$\begin{array}{c}7^{a} \\
N=30\end{array}$} & Tremor & .375 & 1.030 & 0.719 & $-1.746-2.497$ & . & . & . & . \\
\hline & Rigidity & .290 & .634 & 0.651 & $-1.016-1.598$ & . & . & . & . \\
\hline & Walking & .923 & .805 & 0.263 & -.736-.583 & . & . & . & \\
\hline & Balance & -.700 & .295 & 0.026 & -1.309--.092 & . & . & . & . \\
\hline \multirow{4}{*}{$\begin{array}{c}8 \\
N=39\end{array}$} & Tremor & -.242 & .084 & 0.007 & $-.414--.070$ & .061 & .079 & 0.446 & $-.100-.223$ \\
\hline & Rigidity & 1.183 & .680 & 0.091 & -.2012 .566 & -.734 & .637 & 0.257 & $-2.030-.561$ \\
\hline & Walking & 1.186 & .684 & 0.092 & $-.206-2.577$ & -.566 & .640 & 0.383 & $-1.869-.736$ \\
\hline & Balance & .948 & .497 & 0.066 & $-.064-1.96$ & -.457 & .465 & 0.334 & $-1.405-.491$ \\
\hline \multirow{4}{*}{$\begin{array}{c}9 \\
N=46\end{array}$} & Tremor & .251 & .199 & 0.215 & $-.152-.654$ & -.125 & .182 & 0.496 & $-.492-.242$ \\
\hline & Rigidity & 1.518 & .758 & 0.052 & $-.015-3.050$ & -1.514 & .691 & 0.034 & -2.910--.118 \\
\hline & Walking & .758 & .52 & 0.157 & $-.306-1.822$ & -.719 & .479 & 0.141 & $-1.688-.250$ \\
\hline & Balance & .369 & .366 & 0.319 & $-.370-1.109$ & -.362 & .333 & 0.284 & $-1.035-.312$ \\
\hline \multirow{4}{*}{$\begin{array}{c}10 a b \\
N=45\end{array}$} & Tremor & .593 & .414 & 0.161 & $-.246-1.430$ & -.751 & .293 & 0.014 & -1.342--.158 \\
\hline & Rigidity & .290 & .220 & 0.195 & -.155-.735 & -.068 & .155 & 0.666 & -.382-.247 \\
\hline & Walking & .096 & .169 & 0.572 & $-.246-.439$ & .035 & .120 & 0.768 & $-.206-.277$ \\
\hline & Balance & -.085 & .153 & 0.583 & -.395-.225 & .008 & .108 & 0.939 & $-.211-.227$ \\
\hline \multirow{4}{*}{$\begin{array}{c}11 \\
N=35\end{array}$} & Tremor & .276 & .527 & 0.605 & $-.802-1.353$ & .195 & .347 & 0.578 & $-.515-.906$ \\
\hline & Rigidity & .909 & .922 & 0.322 & $-.977-2.796$ & -.669 & .608 & 0.280 & $-1.913-.575$ \\
\hline & Walking & .652 & .896 & 0.472 & $-1.180-2.484$ & -.709 & .591 & 0.239 & $-1.197-.499$ \\
\hline & Balance & .132 & .440 & 0.767 & $-.769-1.032$ & .419 & .290 & 0.160 & $-.175-1.013$ \\
\hline \multicolumn{10}{|c|}{$\begin{array}{l}\mathrm{N}=\text { number of completed questionnaires, Walking }=\text { walking problems, balance }=\text { balance problems. Each } \\
\text { participant received } 50 \text { assessments over the course of } 5 \text { days. Bold }=\text { significant result } p<0.05 \text {. In } 3 \text { subjects ( } 1 \text {, } \\
3 \text { and } 7 \text { ) 'being alone' was removed from the model because of a } 100 \% \text { correlation with 'being at home'. a Subject } \\
\text { suffered from clinically relevant anxiety as objectified by the Parkinson Anxiety Scale }{ }^{20} \text {, b Subject suffered from } \\
\text { clinically relevant depression as objectified by the Beck Depression Inventory- } I^{21} \text {, ' Subject did not experience any } \\
\text { balance problems during ESM data collection (score }=1 \text { ) }\end{array}$} \\
\hline
\end{tabular}


Supplementary figure 1: Moment-to-moment fluctuations in positive and negative affect and in tremor, rigidity, balance problems, walking problems and dyskinesia in individual patients
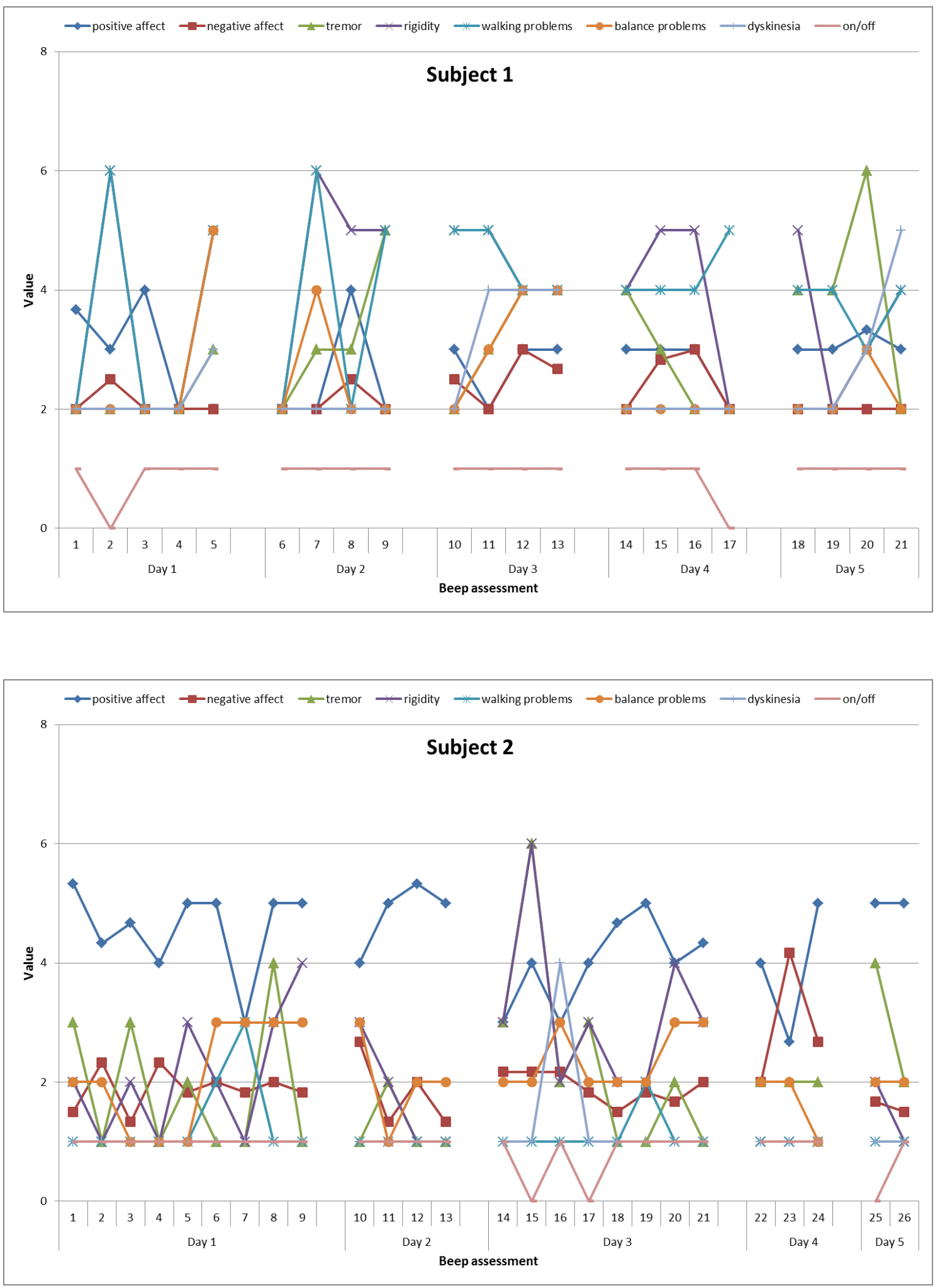

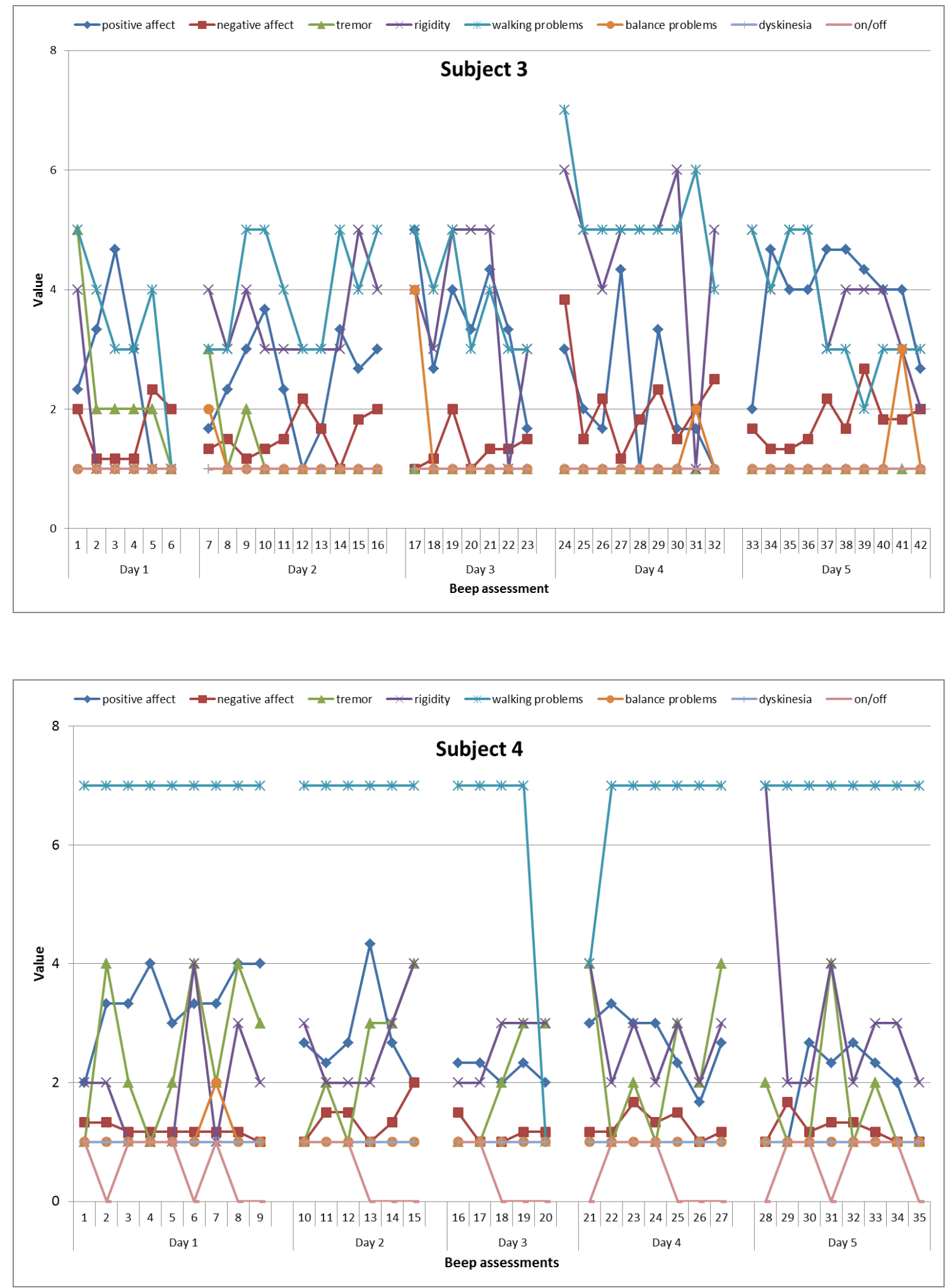

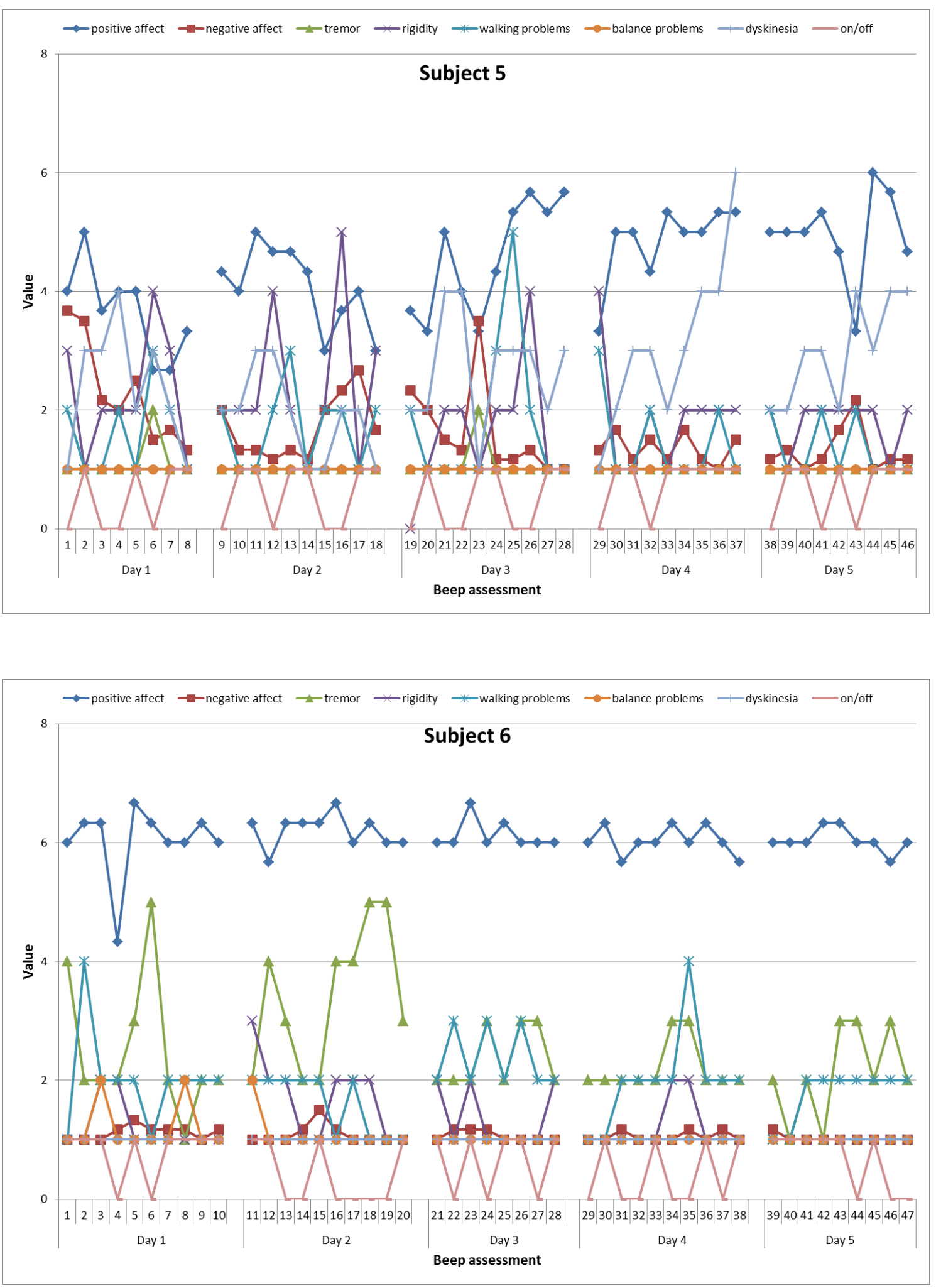

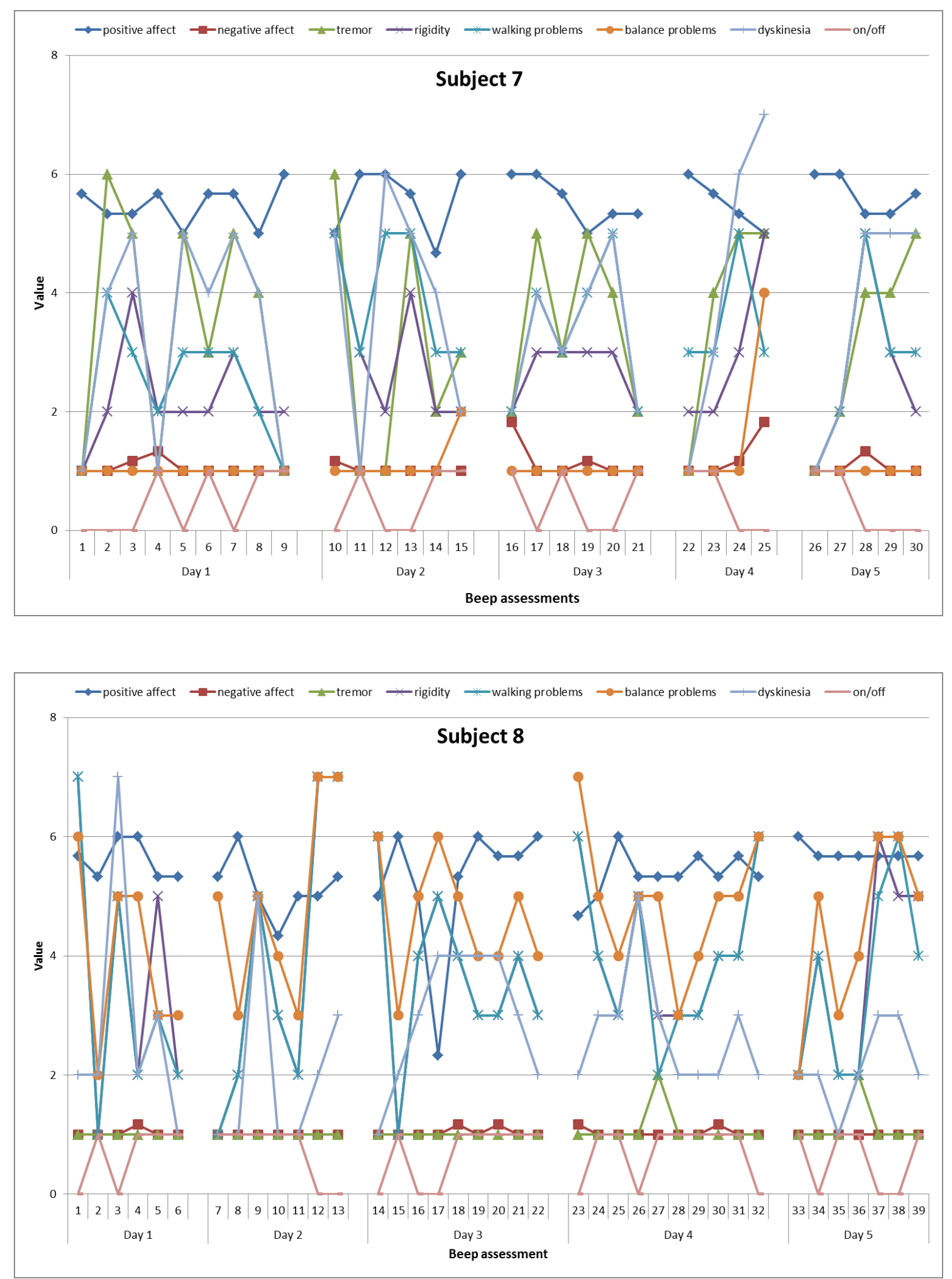

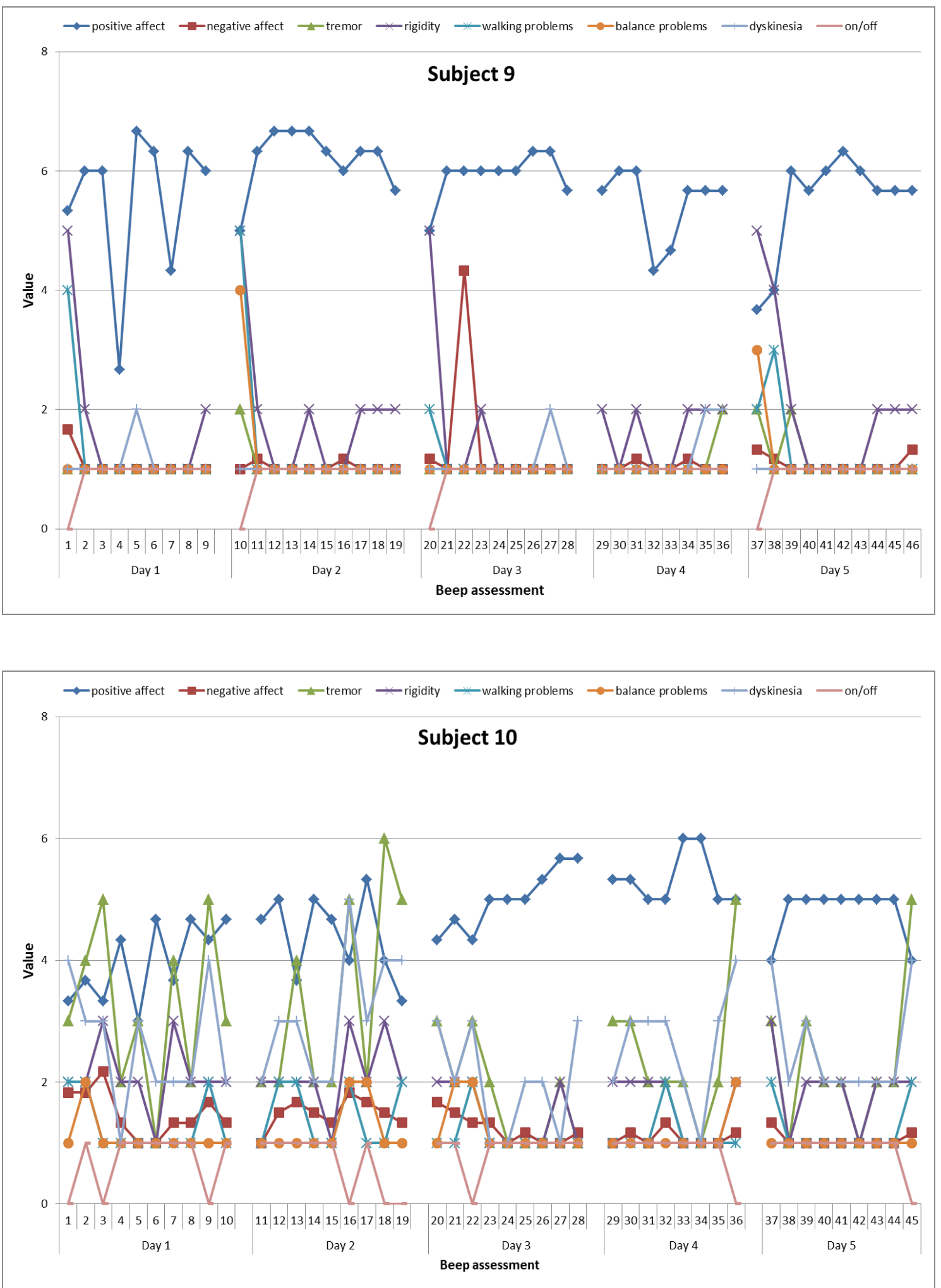


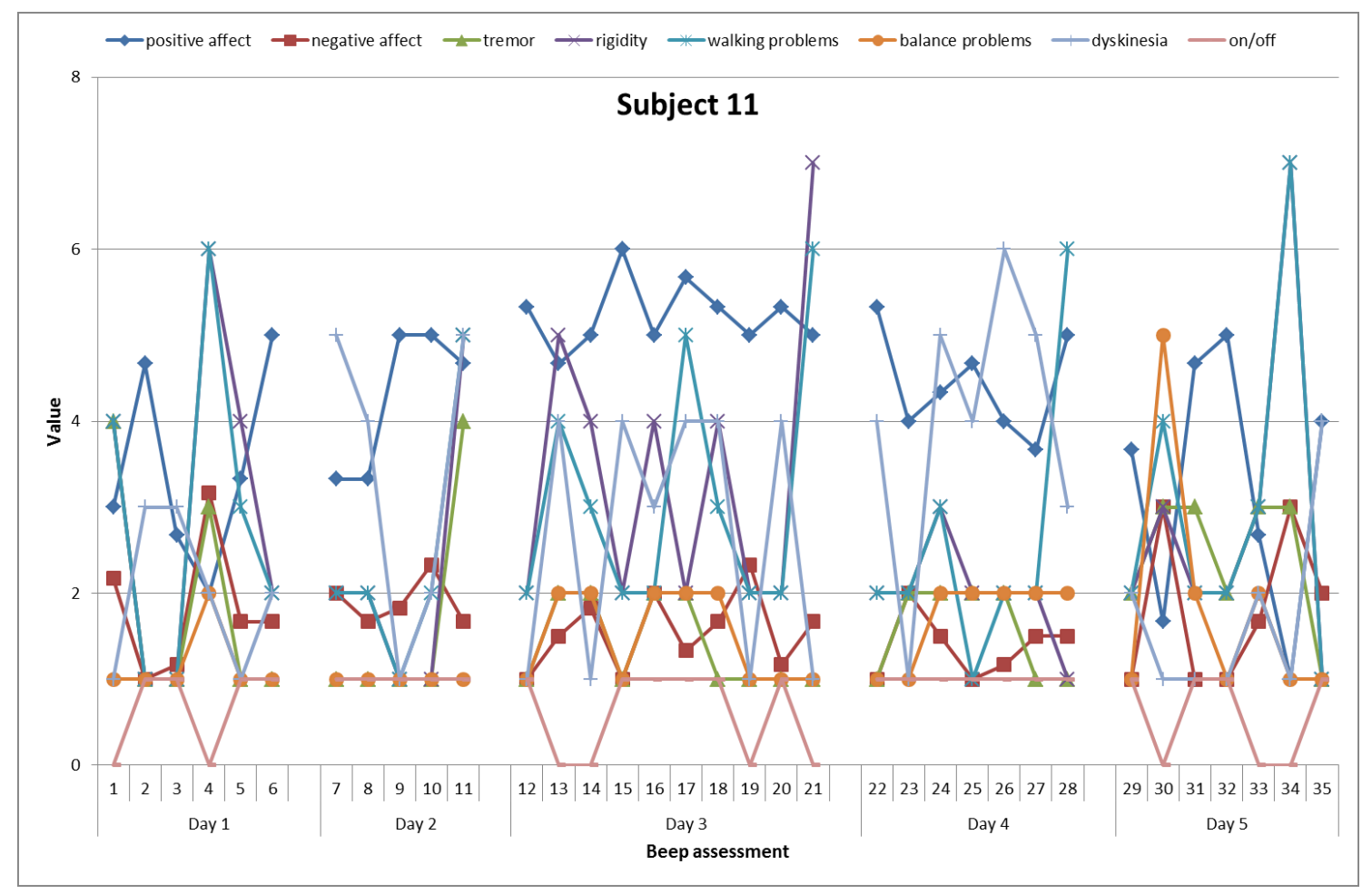





\section{8}

\section{Cognitive behavioural therapy for anxiety}

disorders in Parkinson's disease: Design of a randomised controlled trial to assess clinical effectiveness and changes in cerebral connectivity

Anne Mulders*,Anja Moonen*, Kathy Dujardin, Mark Kuijf, Annelien Duits, Bérengère Flinois, Ron Handels. Renauld Lopes, Albert Leentjens J Psychosom Res 2018;112:32-39.

*These authors equally contributed to this work 


\section{ABSTRACT}

Background: anxiety disorders occur in up to $35 \%$ of patients with Parkinson's disease (PD) and have a negative effect on motor symptoms and quality of life. To date, no clinical trials specifically targeting anxiety in PD patients have been published.

Objective: to describe the rationale and methodology of a randomised controlled trial (RCT) that aims to study the clinical effectiveness, alterations in brain circuitry, and cost-effectiveness of cognitive behavioural therapy (CBT) for anxiety in PD.

Methods: this study is a prospective, two-centre RCT in which sixty PD patients with anxiety will be randomised to CBT treatment and clinical monitoring (intervention group) or to clinical monitoring only (control group). The CBT module used in this study was specifically developed to address symptoms of anxiety in PD patients. Participants will undergo standardised clinical, cognitive and behavioural assessment at baseline and at 2 follow-up measurements, as well as resting-state fMRI and DTI scanning before and after the intervention. The primary outcome measure is changes in severity of anxiety symptoms. Secondary outcome measures involve long-term changes in anxiety symptoms, changes in functional and structural connectivity between limbic and frontal cortices, and cost-effectiveness of the treatment. The study is registered at the ClinicalTrials.gov database under registration number NCT02648737.

Conclusion: this study is the first that evaluates both the clinical effectiveness, costeffectiveness, as well as the biological impact of CBT for anxiety in PD patients that, if proven effective, will hopefully contribute to a better and evidence-based approach for these non-motor symptoms. 


\section{BACKGROUND}

Anxiety disorders occur in up to $35 \%$ of patients with Parkinson's disease (PD) and have a negative effect on several motor symptoms including tremor, gait, dyskinesias, freezing, on-off fluctuations, and on quality of life in general ${ }^{1-3}$. However, anxiety disorders in PD patients are often not recognised and consequently not treated ${ }^{4,5}$.

Neurobiological studies of affective processing have demonstrated the involvement of an extensive frontal-subcortical limbic network ${ }^{6}$, which mainly depends on intact dopaminergic neurotransmission ${ }^{7}$. PD is primarily characterised by the progressive degeneration of dopaminergic systems and anxiety is believed to originate, at least in part, from the underlying pathological process of $\mathrm{PD}^{8,9}$. Indeed, anxiety often appears prior to the appearance or recognition of motor symptoms ${ }^{9}$, and even non-anxious PD patients already show altered emotion regulation compared to healthy adults ${ }^{10}$. However, the fact that anxiety symptoms do not reliably improve by L-DOPA replacement therapy suggests that other mechanisms besides dopamine deficiency are involved in the aetiology of anxiety symptoms in $\mathrm{PD}^{11,12}$, such as the noradrenaline and serotonin systems. Both these systems are implicated in affective processes and known to be substantially affected in the PD process ${ }^{13-15}$. On the other hand, both fears and social implications associated with motor symptoms of PD can provoke or exacerbate anxiety symptoms in $\mathrm{PD}^{16}$. Given this complex interaction between physiological and psychological factors and the diversity of the disease, psychotherapeutic treatment of anxiety requires adaptations that are tailored to the specific needs of PD patients ${ }^{17,18}$.

Cognitive behavioural therapy (CBT) is considered the gold standard in psychotherapeutic treatment of anxiety in the general population ${ }^{19}, 20$. CBT is defined as 'An amalgam of behavioural and cognitive problem-based interventions guided by principles of applied science. The behavioural interventions aim to decrease maladaptive behaviours and increase adaptive ones by modifying their antecedents and consequences and by behavioural practices that result in new learning. The cognitive interventions aim to modify maladaptive cognitions, self-statements, or beliefs $^{21,}{ }^{22}$. In PD, CBT has been proven to be an effective treatment for depression and impulse control disorders (ICD) ${ }^{23-25}$. PD patients who received CBT for depression in a randomised controlled trial reported not only a reduction in depression and 
comorbid anxiety, but also a beneficial influence on coping and quality of life, compared to PD patients who only received clinical monitoring ${ }^{23}$. To date, no clinical trials specifically targeting anxiety in PD patients have been published. However, there have been several pilot studies for CBT and internet-based CBT in PD patients with mixed depressive and anxiety symptoms or mild to moderate depression or anxiety symptoms, respectively ${ }^{25-27}$. Both studies reported significant reductions in depressive symptoms, but not in anxiety symptoms, which may have been due to small sample sizes and lack of power ${ }^{26,27}$. Another uncontrolled study investigating the use of a tailored CBT module for anxiety in 12 PD patients showed that CBT can reduce anxiety levels with persisting benefits at 3 and 6 month follow-up ${ }^{28}$. Although the previous studies are limited by their small sample size, and/or uncontrolled nature, they warrant further exploration of CBT for anxiety in PD patients ${ }^{25}$.

In addition to clinical effectiveness, several imaging studies have demonstrated that psychotherapeutic interventions, including CBT, can modify neural correlates of affective processing ${ }^{29-32}$. CBT-induced increases in both functional ${ }^{30,33}$ and structural connectivity ${ }^{34}$ between limbic and frontal cortices have been demonstrated in patients with social anxiety disorder (SAD), one of the most common anxiety disorders in PD'1. Here, we describe the design of the study Cognitive Behavioural Therapy for Anxiety Disorders in Parkinson's Disease, a two-centre randomised controlled trial (RCT) that aims to examine the clinical effectiveness, cost-effectiveness as well as changes in cerebral connectivity following CBT for anxiety in PD. The main research questions are:

1) Is $C B T$ treatment of anxiety in $P D$ patients more effective than clinical monitoring in terms of change in anxiety levels, quality of life and well-being?

2) Is CBT treatment cost-effective, compared to clinical monitoring?

3) Does successful CBT treatment lead to changes in functional and structural brain connectivity between the frontal and prefrontal cortex and limbic structures such as the amygdala, cingulate gyrus and hippocampus?

\section{METHODS}

\section{Study design}

This study is a prospective, open RCT with PD patients recruited in two centres in Europe (Maastricht University Medical Centre, Maastricht, the Netherlands and Lille 
University Hospital, Lille, France). Sixty PD patients with anxiety and their caregivers (optional) will be randomised to CBT and clinical monitoring (intervention group) or clinical monitoring only (control group). A randomised block design with 6 blocks of 10 participants will be applied. All participants will undergo standardised clinical, cognitive and behavioural assessment at baseline $(t=0)$, at the end of the intervention $(t=1)$ as well as 3 months after the intervention $(t=2)$. Moreover, participants randomised to the intervention group will receive an additional full assessment at 6 month follow-up $(t=3)$. At baseline $(t=0)$ and at the end of the intervention $(t=1)$ participants will undergo Magnetic Resonance Imaging (MRI) scanning, except in case of contraindications for undergoing MRI (e.g., deep brain stimulation, claustrophobia). The duration of the intervention will be approximately $10-12$ weeks. Participants randomised to the control group will be given the option to receive CBT after the 3 month follow-up assessment. This will be done as clinical care and not be part of the study.

\section{Study population}

\section{In- and exclusion criteria}

Sixty patients with idiopathic PD according to the Queens Square Brain Bank criteria ${ }^{35}$ and their caregivers (optional) will be prospectively enrolled. In- and exclusion criteria are listed in Table 1. In order to achieve a representative study sample, participants will be included irrespective of their disease stage or their current antiparkinsonian medication, provided a stable situation is present. In case a caregiver is involved, the caregiver should have daily contact with the study participant and have no serious medical or psychiatric conditions.

\section{Recruitment}

Patients will be recruited from the Movement Disorders clinics in the two participating centres. The treating neurologists will evaluate initial eligibility of the patients. Moreover, flyers with general information about the study and contact details of the researchers will be distributed in Movement Disorders clinics in other hospitals in the region and at several Parkinson support groups. After patients have been informed about the study and agreed to participate, a baseline assessment will take place, in which participants will be further screened to evaluate whether they meet all in- and exclusion criteria. A flowchart of the inclusion procedure is presented in Figure 1. 
Table 1. In- and exclusion criteria

\begin{tabular}{|c|c|c|}
\hline Inclusion criteria & $\begin{array}{l}- \\
- \\
-\end{array}$ & $\begin{array}{l}\text { Idiopathic PD according to the Queens Square Brain Bank } \\
\text { diagnostic criteria }{ }^{35} \\
\text { Presence of clinically relevant anxiety symptoms, as } \\
\text { operationalised by a Parkinson Anxiety Scale (PAS) }{ }^{3} \text { persistent } \\
\text { score > } 9 \text { and/or PAS avoidance score > } 3 \\
\text { Using a stable dose of levodopa or other antiparkinsonian } \\
\text { medication for at least } 1 \text { month } \\
\text { No other current psychological treatment for anxiety; } \\
\text { psychopharmacotherapy is allowed if a stable dose is used at } \\
\text { least } 2 \text { months prior to participation and the patient still meets } \\
\text { inclusion criteria. During the trial the dosage should not be } \\
\text { changed. Medication use and mental health care will be tracked } \\
\text { throughout the study. } \\
\text { Age between } 35 \text { and } 80 \text { years old }\end{array}$ \\
\hline Exclusion criteria & $\begin{array}{l}- \\
- \\
- \\
-\end{array}$ & $\begin{array}{l}\text { Parkinsonian syndromes or neurodegenerative disorders other } \\
\text { than PD } \\
\text { Dementia or severe cognitive decline, operationalised as a } \\
\text { Montreal Cognitive Assessment (MoCa) })^{36} \text { score }<24 \\
\text { Major depressive disorder (MDD) as defined by the DSM } 5 \\
\text { criteria }^{37} \\
\text { Abuse of alcohol, drugs or benzodiazepines }\end{array}$ \\
\hline
\end{tabular}

\section{Randomisation, blinding and treatment allocation}

After informed consent is obtained and the baseline measurement is performed, participants will be randomly assigned to one of two groups: the intervention group or control group. Randomisation will be performed over both centres together in order to compensate for between-centre differences in population and involves a randomised block design with 6 blocks of 10 participants. Randomisation is performed by the coordinating investigator using the website randomization.com. Assessments will be performed by a psychologist who is not involved with the treatment and blinded for the intervention. The participants will be instructed not to comment on the intervention they received. 


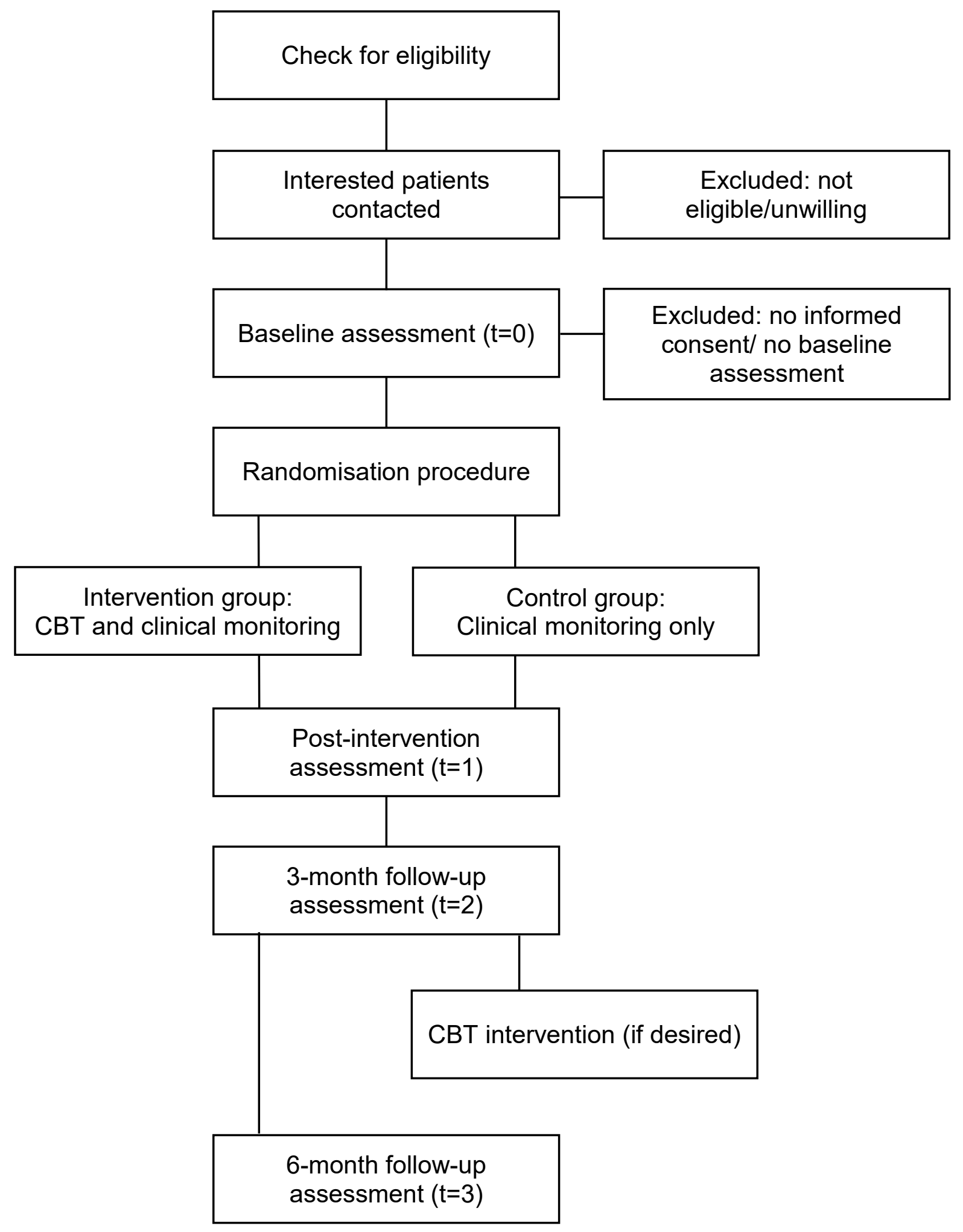

Figure 1. Study flow chart. 


\section{Intervention}

\section{CBT Treatment}

Participants randomised to the intervention group will receive CBT and clinical monitoring. The CBT consists of 10 weekly individual sessions of 60 - 75 minutes, tailored to the preferences and needs of each patient. In each session, a registered psychologist will address specified aspects of (coping with) anxiety and related concerns with a specific focus on behaviour and thoughts associated with anxiety. Any other neuropsychiatric symptoms that are present, such as depressive symptoms or apathy, will be addressed as well, although the main focus will be on anxiety.

The content of the CBT module was based on existing literature of CBT treatment of generalised anxiety disorder and social anxiety disorder and on published existing modules for CBT in anxiety and depression. Published existing modules include amongst others: psycho-education and awareness, motivational interviewing, behaviour activation, thought monitoring and restructuring, exposure, relaxation techniques, coping and problem solving skills, exercise, and sleep hygiene ${ }^{23,26,38-40 .}$ We adjusted these existing modules to better serve the specific needs, concerns, and circumstances of PD patients with anxiety. The resulting module was subsequently presented to small groups of PD patients and their caregivers (in 'focus-groups'), as well as to representatives of the scientific section of the Dutch Parkinson Patient Foundation (Parkinsonpatiëntenvereninging) and to PD experts in both countries, in order to gather patient and professional feedback, respectively. The final CBT module, which consists of a treatment manual for therapists and a workbook for patients, was originally written in English and later translated to French and Dutch.

All CBT sessions have a similar overall structure. Each session will start with setting the agenda for that particular session, after which the home assignments will be reviewed (in all but the first session). Then the new topic will be introduced and exercises related to the new topic will be practiced. Subsequently, the new home assignments for the upcoming week will be discussed as well as possible barriers or concerns for completing the new tasks and/or tasks from previous sessions. The first and last session will have some additional components related to the introduction and termination of the therapy, respectively. An overview of the sessions is provided in Table 2. 
Table 2. Overview of the Cognitive Behavioural Therapy sessions ${ }^{a}$

\begin{tabular}{ll}
\hline Session & \\
\hline 1 & Introduction and psycho-education about anxiety \\
2 & Monitoring anxiety \\
3 & Management of anxiety: introduction and the importance of self-management \\
4 & Management of anxiety: diaphragmatic breathing and mindful awareness \\
5 & Changing thoughts \\
6 & Management of anxiety: progressive muscle relaxation and imagery \\
7 & Problem solving \\
8 & Changing anxiety behaviour \\
9 & Review session \\
10 & Self-management plan and closure \\
& Booster session
\end{tabular}

${ }^{a}$ A detailed treatment protocol and therapist manual have been developed. Moreover,
participants will receive a workbook with handouts and worksheets. ${ }^{b}$ Each patient will
receive a booster session 1.5 month following the final treatment session. The aim of the
booster session is the monitor the patient's progress, to provide clinical assistance if
necessary, and to encourage continued use of coping skills.

Home assignments are an important part of CBT. In order to override dysfunctional patterns that promote anxiety and achieve change, repeated practice in daily life is necessary. Patients will receive a workbook during the first session. Each week the workbook will be supplemented with handouts and worksheets that correspond to the current topic. Even though patients with severe cognitive impairment (MoCa score <24) will be excluded from participation, cognitive problems are common in PD patients ${ }^{41}$, and probably a substantial proportion of the participants will experience cognitive problems to some degree. These usually relate to deficits in attention and executive function, although memory deficits and visuospatial functions are also frequent 40 . Therefore, all sessions and accompanying handouts and worksheets have a clear structure and the psychologist will always check whether the information and assignments are clear to the participant and whether there are any barriers or concerns for completing the home assignments. If necessary, the psychologists will problemsolve any issues. The therapy sessions will take place at the outpatients of the Movement Disorders Clinics of the two participating centres. In case medical or other 
reasons make it difficult for the patient to visit the clinics, the treating therapist can visit the participants at home.

\section{Clinical monitoring}

Clinical monitoring has been recommended as a control situation when exploring the clinical effectiveness of a new or adjusted psychotherapeutic intervention ${ }^{43}$. All patients will receive clinical monitoring; those not randomised to the intervention group will receive clinical monitoring only. Clinical monitoring involves a phone call by an independent psychologist one month following the baseline assessment to inquire about current anxiety symptoms. Moreover, participants will receive general education material on coping with anxiety, derived from the Dutch/French psychiatric association webpages. Participants in the control group will remain under the care of their personal physicians, who will also monitor their medical and psychiatric status. During the intervention and follow-up period, all participants are asked to give notice when starting with any other form of psychotherapeutic interventions or psychopharmacotherapy for their anxiety. Those patients randomised to the control condition of clinical monitoring only, will be given the opportunity to receive CBT after the 3 month follow-up assessment. This treatment will be part of regular patient care and is covered by the patient's health insurance. Hence, it will not be part of the study.

\section{Outcome measures}

The primary outcome for studying the clinical effectiveness of the CBT treatment is the difference between the intervention and control group in anxiety score changes between baseline $(t=0)$ and post-treatment $(t=1)$ as measured with the Hamilton Anxiety Rating Scale (HARS) ${ }^{42}$. Secondary outcome measures involve long-term clinical effectiveness, which will be assessed similarly, but then using the 3-month $(\mathrm{t}=$ 2 ) and 6-month follow-up anxiety scores ( $t=3$, intervention group only). Moreover, changes in functional and structural cerebral connectivity between the frontal and prefrontal cortex and limbic structures such as the amygdala, cingulate gyrus and hippocampus before and after the intervention will be studied, as measured with resting-state MRI (rs-fMRI) and Diffusion Tensor Imaging (DTI). The difference in effectiveness between CBT and clinical monitoring in terms of disease-specific quality of life, generic health-related quality of life and well-being will be assessed using the Parkinson's Disease Questionnaire-8 (PDQ-8) ${ }^{45}$, the EQ-5D-5L ${ }^{46}$ and Icepop 
Capability measure for older people (ICECAP-O $)^{47}$, respectively. Lastly, care resource utilisation will be obtained by an adapted version of the Resource Utilisation in Dementia ${ }^{48}$ questionnaire. Other study parameters involve changes in comorbid psychiatric symptoms, motor symptoms, sleep disturbance, coping, as well as caregiver burden. An overview of all questionnaires is provided in Table 3 .

Table 3. Questionnaires administered at t0, $\mathrm{t} 1, \mathrm{t} 2$ and $\mathrm{t} 3$

\begin{tabular}{|c|c|}
\hline Domain & Instrument \\
\hline Anxiety & $\begin{array}{l}\text { Hamilton Anxiety Rating Scale }{ }^{44}, \text { Parkinson Anxiety Scale }{ }^{3} \text {, } \\
\text { Liebowitz Social Anxiety Scale }{ }^{49} \text {, Mini International } \\
\text { Neuropsychiatric Inventory section G and } \mathrm{O}^{50}\end{array}$ \\
\hline Global cognitive status & Montreal Cognitive Assessment ${ }^{36}$ \\
\hline Depression & Hamilton Depression Rating Scale ${ }^{51}$ \\
\hline Apathy & Lille Apathy Rating Scale 52 \\
\hline Health-related quality of life & Parkinson's Disease Questionnaire ${ }^{45}, E Q-5 D-5 L^{c 46}$ \\
\hline Well-being & ICECAP-O ${ }^{c 47}$ \\
\hline $\begin{array}{l}\text { Sleep and nocturnal } \\
\text { disturbances }\end{array}$ & Parkinson's Disease Sleep Scale $2^{53}$ \\
\hline Coping strategies & Brief Cope scale ${ }^{54}$, Thought Control Questionnaire ${ }^{55}$ \\
\hline $\begin{array}{l}\text { Health-related costs, } \\
\text { patient and family related } \\
\text { costs }\end{array}$ & Adapted version of the Resource Utilisation in Dementia ${ }^{\mathrm{d} 48}$ \\
\hline $\begin{array}{l}\text { Motor symptom severity } \\
\text { and PD disease stage }\end{array}$ & MDS-UPDRS, Hoehn \& Yahr staging ${ }^{56}$ \\
\hline Caregiver burden & Zarit burden interview ${ }^{57}$ \\
\hline \multicolumn{2}{|c|}{$\begin{array}{l}\text { ICECAP-O = Icepop Capability measure for older people, MDS-UPDRS = Movement } \\
\text { Disorder Society - Unified Parkinson's disease rating scale, PD = Parkinson's disease. }{ }^{a} \\
t=0 \text { : baseline measurement, } t=1 \text { : post-intervention measurement, } t=2: 3-\text { month follow up. }{ }^{b} \\
t=3: 6 \text {-month follow-up for intervention group only, }{ }^{c} \text { Will be administered to both } \\
\text { participants and caregivers, }{ }^{d} \text { In this version the formulation of items has been adjusted to } \\
\text { PD instead of dementia and two questions regarding the use of speech therapy and home } \\
\text { aids were added. }\end{array}$} \\
\hline
\end{tabular}

The demographic and disease-related variables that will be collected during baseline include sex, date of birth, years of formal education, year of PD onset, side of onset, year of PD diagnosis, details on Parkinson medication (i.e., drug name, dose, frequency, levodopa equivalence) and other medication including psycho- 
pharmacology, alcohol use, medical history (i.e., cerebrovascular diseases, psychiatric disorders) and family medical history (i.e., PD, other neurological disorders, dementia, psychiatric disorders). Any change in Parkinson medication will be tracked during the whole study period.

\section{MRI scan}

MRI will be acquired using a 3T whole-body scanner (Achieva TX, Philips Healthcare, Best, the Netherlands) using a 32-channel head coil for signal reception. The MRI protocol includes an anatomical scan, rs-fMRI, DTI and B0 field map. The total MRI scan will take about 45 minutes. Details of the brain MRI protocol can be found in Table 4. Imaging will be performed at the Department of Radiology of Maastricht University Medical Centre and at the Department of Radiology of Lille University Hospital.

\section{Data collection and management}

All personnel involved in the data collection will review the standard operating procedures (SOP) and manuals. Assessors are certified in Good Clinical Practice (GCP) and in performing the MDS-UPDRS part $1 \mathrm{II}^{56}$. Data will be collected on paper forms and entered into a web-based data entry portal of which a back-up will be stored electronically on a daily basis. The data of all participants will be handled confidentially. A subject identification code list will be used to trace data to an individual subject. The coordinating investigator will provide all data of subjects with a number that is not based on the patient's initials and birth-date. The key to the code will be safeguarded by the coordinating investigator. All data will be stored for 15 years as required by law.

Table 4. Brain MRI protocol

\begin{tabular}{lcccc}
\hline Sequences & $\begin{array}{c}\text { 3D T1-weighted } \\
\text { MP-RAGE }\end{array}$ & $\begin{array}{c}\text { Resting-state } \\
\text { fMRI }\end{array}$ & $\begin{array}{c}\text { Diffusion } \\
\text { tensor imaging }\end{array}$ & B0 Field map \\
\hline Field of view $(\mathrm{mm})$ & $240 \times 240$ & $192 \times 192$ & $256 \times 256$ & $256 \times 256$ \\
Matrix & $256 \times 256$ & $64 \times 64$ & $128 \times 128$ & $128 \times 128$ \\
Slices & 231 & 40 & 66 & 66 \\
Inter-slicegap $(\mathrm{mm})$ & 0 & 0 & 0 & 0 \\
Voxel $\left(\mathrm{mm}^{3}\right)$ & $0.65 \times 0.65 \times 0.65$ & $3 \times 3 \times 3$ & $2 \times 2 \times 2$ & $2 \times 2 \times 2$ \\
TR/TE $\left(\mathrm{ms}^{2}\right)$ & $12 / 3.3$ & $2400 / 30$ & $12000 / 56$ & $660 / 4$ \\
Flip angle & $9^{\circ}$ & $90^{\circ}$ & $90^{\circ}$ & $80^{\circ}$ \\
B value $\left(\mathrm{ms} / \mathrm{mm}^{2}\right)$ & 1000 & \\
$\mathrm{Nr}$ of directions & 32 & \\
\hline $\mathrm{Nr}=$ Number, fMRI = functional Magnetic Resonance Imaging \\
\hline
\end{tabular}




\section{Power and sample size}

Power calculation was based on a standardised difference of 0.8 in HARS total score, with alpha set at 0.05 and power set at 0.80 , and a predicted effect size of Cohen's $d$ (0.95), based on a previous RCT with CBT in $\mathrm{PD}^{23}$. The standardised difference is defined as the ratio of the difference of interest to the standard deviation in scores of the sample. The difference of interest was set 3 points on the HARS (being the difference in average scores between patients without anxiety and with mild anxiety2; standard deviation was estimated at 52, 23. Based on these quantities, the required number of patients to be included is 40 (20 per group). Including 30 patients per group would allow for a drop-out of approximately $30 \%$. This is a conservative sample size calculation since, based on the only published RCT of $C B T$ in depression in $\mathrm{PD}^{23}$, we expect that the effect size will be larger ( $>5$ points) and drop-out will be lower $(<10 \%)$. If subjects decide to leave the study prematurely they will not be replaced by new participants. The dropped out participants will be included in the analysis according to the intention-to-treat principle (ITT).

No sample size calculation was made for the MRI analyses specifically, since Iongitudinal data to determine the sensitivity of different DTI parameters to change over time are lacking. However, taking the sample size of published studies in this area as reference $32,34,58,59$, we expect to be overpowered for these analyses using the same sample size as for the analysis of clinical efficacy.

\section{Statistical analyses}

Unless indicated otherwise, analysis will be performed using IBM SPSS statistics 24.0, Stata 13 , or more recent versions if available. Significance level of 0.05 will be used. The numerical variables will be described as means, median, standard deviations and ranges. Categorical variables will be described as frequencies and percentages. Data analyses of primary and secondary outcomes will be conducted using relevant univariate, multivariate and multilevel techniques.

For the primary study parameter, between-group comparisons will be conducted on post-treatment data and 3 month follow-up data in order to compare within-subject changes in anxiety scores (HARS) relative to baseline between the intervention and control group. This will be done using separate repeated measures Analysis of 
Covariance (rm-ANCOVA) for each of the two outcomes. Covariates include the use of benzodiazepines and antidepressants, Parkinson medication, which will be converted into levodopa equivalents using the algorithm of Tomlinson et al ${ }^{60}$, as well as significant differences in other relevant parameters.

For the secondary parameters, between-group comparisons will be likewise conducted on post-treatment data and 3-months follow-up data to assess differences in changes relative to baseline between the intervention- and control group. To compare changes in anxiety scores and the secondary parameters between baseline and 66months follow-up in the intervention group, within-subject comparisons will be performed.

For MRI scan analyses, between-group analyses using paired-sample and two-sample t-tests will be conducted to calculate differences in changes in cerebral connectivity pre- and post-intervention. Functional connectivity measures and graph topology indices will be extracted from rs-fMRI data ${ }^{61}$. Diffusion metrics (fractional anisotropy, radial and axial diffusivity) and structural connectivity computed from DTI data will be also used as descriptive variables in the statistical comparisons ${ }^{62}$.

The economic evaluation will be performed following the Dutch guideline for economic evaluations in healthcare. The incremental cost-effectiveness ratio (ICER) will be calculated using standard unit price based costs in combination with the adapted RUD and EQ-5D-5L utility based QALYs. An ITT analysis, a 3-month time horizon and a societal perspective will be adopted. A bootstrap analysis will be used to reflect the uncertainty in this ICER.

\section{Drop-outs, missing values and adverse events}

Participants can leave the study at any time for any reason or no reason at all if they wish to do so without any consequences. The investigator can decide to withdraw a subject from the study for urgent medical reasons. If subjects decide to leave the study prematurely they will not be replaced by new participants. The dropped out participants will be included in the analysis according to the ITT. For the ITT analyses, missing values on the primary outcome will be imputed using multiple imputations. For this, ten imputed datasets will be generated using multivariate multiple imputation by chained equations (MICE) $)^{63}$ with demographic, clinical and baseline values of the primary 
outcome as predictors of the missing outcome values as well as variables predicting missingness (e.g. age, illness severity, morbidity). This technique yields better (i.e. less biased) estimates for outcome analyses in RCTs than single imputation or complete case analysis with covariate adjustment for missingness, especially if data are notmissing-at-random (i.e. missingness is related to unobserved variables) ${ }^{64}$. Subsequent analyses on imputed datasets will be performed in state 13 or higher ${ }^{65}$.

Adverse events are defined as any undesirable experience occurring to a subject during the study, which may or may not be related to the trial procedure or experimental intervention. All spontaneously reported study-related adverse events by the subject or observed by the investigator or his staff will be recorded according to section 10 , subsection 1 of the Dutch Research Involving Human Subjects Act (WMO). In case of a serious adverse event the sponsor, the Ethics committee and other relevant authorities such as the principal investigator and Toetsingonline will be notified immediately. A liability insurance which is in accordance with the legal requirements in the Netherlands (Article 7 WMO) and France has been signed.

\section{Medication adjustments}

Preferably, Parkinson medication is kept stable during the study. Nonetheless, if medication changes are presumed necessary by the treating neurologist, they are allowed to do so. Any changes in medication will be tracked during the intervention period and follow-up moments. Psychopharmacotherapy is allowed if, by the start of the study, a stable dose is used at least 2 months prior to participation and the patient still meets the inclusion criteria.

\section{Ethical approval, trial registration and monitoring}

The study is carried out in compliance with the Helsinki Declaration. The local ethics committee of Maastricht University Medical Centre (July, 2016) and Lille University Hospital (September, 2016) have approved the study protocol. Written informed consents will be obtained from all participants. The study is registered at the ClinicalTrials.gov database under registration number NCT02648737, as well at FoxTrialFinder under ID number 004701. The study will be monitored in both centres by Clinical Trial Center Maastricht according to the international ICH-GCP guidelines. 


\section{DISCUSSION}

The overall aim of this open, two-centre RCT is to evaluate the clinical effectiveness of a CBT module for the treatment of anxiety in patients with PD and provide additional information on cost-effectiveness and the underlying changes in functional and structural brain connectivity that occur during this treatment. The treatment module is specifically designed to serve the needs, concerns, and circumstances of PD patients with anxiety and is based on the knowledge and experience of PD experts. CBT will, if proven effective, provide PD patients with behavioural and anxiety management techniques that may give lasting benefits on anxiety symptoms, well-being, quality of life, and possibly on motor symptoms. In this multinational RCT design patients are recruited in two countries at both outpatient clinics and informal meeting points for PD patients. This way of recruiting facilitates generalizability, as a broader range of patients can be reached instead of solely patients with more severe PD symptoms that come from the Movement Disorders Clinics.

While most studies investigating brain alterations following psychotherapeutic interventions use functional imaging methods, only few have examined structural brain correlates of $\mathrm{CBT}^{30}, 31,34$. One study using structural and diffusion MRI measures observed reductions in parieto-occipital and prefrontal grey matter volumes, increases in fractional anisotropy in two anxiety-related white matter fiber tracts, as well as increases in structural connectivity in a frontolimbic network following a 10-week groupbased CBT protocol for SAD patients ${ }^{34}$. Albeit in the absence of a control group and in a different patient population, this study provided insight into how the brain adapts to psychotherapeutic treatments. Our study is the first one that aims at assessing both functional and structural brain alterations following CBT in PD patients using different MRI modalities, such as rs-MRI and DTI, in the presence of a control group. Therefore, this study has an additional value to earlier CBT trials in PD patients ${ }^{23,26,28}$, as it may provide increased understanding of the underlying biological mechanisms of both anxiety and response to treatment in PD patients. Moreover, it can provide additional insight into whether CBT effects differ between different anxiety disorders and patient populations.

There are, however, also some limitations in this study design. Firstly, the follow-up duration of 3 months is relatively short to evaluate the long-term benefits of the CBT 
treatment. However, we agreed it was ethically not acceptable to deny the control group CBT treatment for a period longer than 6 months following the start of the intervention. Alternatively, we added an additional follow-up moment $(\mathrm{t}=3)$ for patients randomised to the intervention group 6 months after the CBT treatment, which will give the possibility to study within-subject changes over time and provide insight into longterm benefits of the CBT treatment. Secondly, although there are no major risks associated with participation in the CBT intervention, participating in the study can be time-consuming and demanding for participants, especially considering the relatively high health care utilisation in PD patients. Some PD patients might not be able to travel independently and have to rely on others, such as partners, family members, friends or other caregivers. These factors make inclusion and treatment adherence challenging. We discussed the feasibility of the number of sessions in multiple PD focus groups and none of the participants, neither in the Netherlands nor in France, perceived the frequency of the sessions as too burdensome or as a reason not to participate. In case medical or other practical reasons hinder participants to visit the outpatient clinic, the therapist can visit the participant at the site where the participant resides. We do not expect this to influence the treatment. Thirdly, with the involvement of different therapists, there is a risk of therapist effects, which refers to the tendency of different therapist to obtain differential symptomatic change in the patients ${ }^{66}$. However, all of our therapists have received appropriate training, and a detailed treatment protocol and therapist manual have been developed, which has shown to minimise therapist effects ${ }^{67,68}$. Despite our efforts to standardise the intervention as much as possible, the involvement of a caregiver during the CBT intervention might likewise moderate treatment outcome as it is optional and not necessary for participation in the study. The purpose of their attendance is primarily to offer caregivers the opportunity to learn about the treatment in which the participant is involved and to explore strategies through which they can support the participant if necessary. This can vary from motivating the participant to do the home assignments up to assisting them to fill out the worksheets in case physical reasons make this difficult. The caregiver attendance is limited to three educational sessions.

In conclusion, this low risk intervention study has great relevance for clinical practice. It evaluates the clinical effectiveness of a CBT module for anxiety in PD and contributes 
CHAPTER 8 | CBT FOR ANXIETY IN PD: RCT

to a better understanding of the underlying biological mechanisms of anxiety in PD. Inclusion started March 2017 and we aim to complete the study in 2019. 


\section{REFERENCES}

1. Broen MP, Narayen NE, Kuijf ML, Dissanayaka NN, Leentjens AF. Prevalence of anxiety in Parkinson's disease: A systematic review and meta-analysis. Mov Disord 2016;31(8):1125-1133.

2. Leentjens AF, Dujardin K, Marsh L, Richard IH, Starkstein SE, Martinez-Martin P. Anxiety rating scales in Parkinson's disease: a validation study of the Hamilton anxiety rating scale, the Beck anxiety inventory, and the hospital anxiety and depression scale. Mov Disord 2011;26(3):407-415.

3. Leentjens AF, Dujardin K, Pontone GM, Starkstein SE, Weintraub D, Martinez-Martin P. The Parkinson Anxiety Scale (PAS): development and validation of a new anxiety scale. Mov Disord 2014;29(8):1035-1043.

4. Pontone GM, Williams JR, Anderson KE, et al. Prevalence of anxiety disorders and anxiety subtypes in patients with Parkinson's disease. Mov Disord 2009;24(9):1333-1338.

5. Reijnders JS, Ehrt U, Weber WE, Aarsland D, Leentjens AF. A systematic review of prevalence studies of depression in Parkinson's disease. Mov Disord 2008;23(2):183-189; quiz 313.

6. Phillips ML, Drevets WC, Rauch SL, Lane R. Neurobiology of emotion perception I: The neural basis of normal emotion perception. Biol Psychiatry 2003;54(5):504-514.

7. Moonen AJH, Wijers A, Dujardin K, Leentjens AFG. Neurobiological correlates of emotional processing in Parkinson's disease: A systematic review of experimental studies. J Psychosom Res 2017;100:65-76.

8. Pontone GM. Anxiety in Parkinson's: a complex syndrome of non-dopaminergic and dopaminergic etiology. Eur J Neurol 2017;24(4):541-542.

9. Djamshidian A, Friedman JH. Anxiety and depression in Parkinson's disease. Curr Treat Options Neurol 2014;16(4):285.

10. Moonen $\mathrm{AJH}$, Weiss $\mathrm{PH}$, Wiesing $\mathrm{M}$, et al. An fMRI study into emotional processing in Parkinson's disease: Does increased medial prefrontal activation compensate for striatal dysfunction? PLoS One 2017;12(5):e0177085.

11. Kim HJ, Park SY, Cho YJ, et al. Nonmotor symptoms in de novo Parkinson disease before and after dopaminergic treatment. J Neurol Sci 2009;287(1-2):200-204.

12. Eskow Jaunarajs KL, Angoa-Perez M, Kuhn DM, Bishop C. Potential mechanisms underlying anxiety and depression in Parkinson's disease: consequences of I-DOPA treatment. Neurosci Biobehav Rev 2011;35(3):556-564.

13. Maillet A, Krack P, Lhommee E, et al. The prominent role of serotonergic degeneration in apathy, anxiety and depression in de novo Parkinson's disease. Brain 2016;139(Pt 9):2486-2502.

14. Remy P, Doder M, Lees A, Turjanski N, Brooks D. Depression in Parkinson's disease: loss of dopamine and noradrenaline innervation in the limbic system. Brain 2005;128(Pt 6):1314-1322.

15. Schapira AHV, Chaudhuri KR, Jenner P. Non-motor features of Parkinson disease. Nat Rev Neurosci 2017;18(8):509.

16. Coakeley S, Martens KE, Almeida QJ. Management of anxiety and motor symptoms in Parkinson's disease. Expert Rev Neurother 2014;14(8):937-946.

17. Pachana NA, Egan SJ, Laidlaw K, et al. Clinical issues in the treatment of anxiety and depression in older adults with Parkinson's disease. Mov Disord 2013;28(14):1930-1934.

18. Dissanayaka NN, White E, O'Sullivan JD, et al. Characteristics and treatment of anxiety disorders in Parkinson's disease. Movement Disorders Clinical Practice 2015;2(2):155-162.

19. Cuijpers P, Sijbrandij M, Koole S, Huibers M, Berking M, Andersson G. Psychological treatment of generalized anxiety disorder: a meta-analysis. Clin Psychol Rev 2014;34(2):130-140.

20. Otte C. Cognitive behavioral therapy in anxiety disorders: current state of the evidence. Dialogues Clin Neurosci 2011;13(4):413-421.

21. Arch JJ, Craske MG. First-line Treatment: A Critical Appraisal of Cognitive Behavioral Therapy Developments and Alternatives. Psychiat Clin N Am 2009;32(3):525-+.

22. Craske M. Cognitive-Behavioral Therapy. New York, NY: APA Books, 2010.

23. Dobkin RD, Menza M, Allen LA, et al. Cognitive-behavioral therapy for depression in Parkinson's disease: a randomized, controlled trial. Am J Psychiatry 2011;168(10):1066-1074. 
24. Okai D, Samuel M, Askey-Jones S, David AS, Brown RG. Impulse control disorders and dopamine dysregulation in Parkinson's disease: a broader conceptual framework. Eur J Neurol 2011;18(12):1379-1383.

25. Koychev I, Okai D. Cognitive-behavioural therapy for non-motor symptoms of Parkinson's disease: a clinical review. Evidence-based mental health 2017;20(1):15-20.

26. Calleo JS, Amspoker AB, Sarwar Al, et al. A Pilot Study of a Cognitive-Behavioral Treatment for Anxiety and Depression in Patients With Parkinson Disease. J Geriatr Psychiatry Neurol 2015;28(3):210-217.

27. Kraepelien M, Svenningsson $\mathrm{P}$, Lindefors $\mathrm{N}$, Kaldo V. Internet-based cognitive behavioral therapy for depression and anxiety in Parkinson's disease - A pilot study. Internet Interventions 2015;2(1):1-6.

28. Dissanayaka NNW, Pye D, Mitchell LK, et al. Cognitive Behavior Therapy for Anxiety in Parkinson's Disease: Outcomes for Patients and Caregivers. Clin Gerontol 2017;40(3):159-171.

29. Bruhl AB, Delsignore A, Komossa K, Weidt S. Neuroimaging in social anxiety disorder-a metaanalytic review resulting in a new neurofunctional model. Neurosci Biobehav Rev 2014;47:260-280.

30. Mansson KNT, Salami A, Frick A, et al. Neuroplasticity in response to cognitive behavior therapy for social anxiety disorder. Transl Psychiat 2016;6.

31. Mansson KNT, Salami A, Carlbring P, Boraxbekk CJ, Andersson G, Furmark T. Structural but not functional neuroplasticity one year after effective cognitive behaviour therapy for social anxiety disorder. Behav Brain Res 2017;318:45-51.

32. Wang $T$, Huang $X$, Huang $P$, et al. Early-stage psychotherapy produces elevated frontal white matter integrity in adult major depressive disorder. PLoS One 2013;8(4):e63081.

33. Mansson KN, Carlbring P, Frick A, et al. Altered neural correlates of affective processing after internet-delivered cognitive behavior therapy for social anxiety disorder. Psychiatry Res 2013;214(3):229-237.

34. Steiger VR, Bruhl AB, Weidt $S$, et al. Pattern of structural brain changes in social anxiety disorder after cognitive behavioral group therapy: a longitudinal multimodal MRI study. Mol Psychiatry 2017;22(8):1164-1171.

35. de Rijk MC, Rocca WA, Anderson DW, Melcon MO, Breteler MM, Maraganore DM. A population perspective on diagnostic criteria for Parkinson's disease. Neurology 1997;48(5):1277-1281.

36. Nasreddine ZS, Phillips NA, Bedirian V, et al. The Montreal Cognitive Assessment, MoCA: a brief screening tool for mild cognitive impairment. J Am Geriatr Soc 2005;53(4):695-699.

37. American Psychiatric Association. Diagnostic and statistical manual of mental disorders. 5th ed. Wahington, DC2013.

38. Stanley MA, Wilson NL, Amspoker AB, et al. Lay Providers Can Deliver Effective Cognitive Behavior Therapy for Older Adults with Generalized Anxiety Disorder: A Randomized Trial. Depress Anxiety 2014;31(5):391-401.

39. Stanley MA, Wilson NL, Novy DM, et al. Cognitive Behavior Therapy for Generalized Anxiety Disorder Among Older Adults in Primary Care A Randomized Clinical Trial. Jama-J Am Med Assoc 2009;301(14):1460-1467.

40. Veazey C, Cook KF, Stanley M, Lai EC, Kunik ME. Telephone-Administered Cognitive Behavioral Therapy: A Case Study of Anxiety and Depression in Parkinson's Disease. J Clin Psychol Med S 2009;16(3):243-253.

41. Yarnall AJ, Breen DP, Duncan GW, et al. Characterizing mild cognitive impairment in incident Parkinson disease: the ICICLE-PD study. Neurology 2014;82(4):308-316.

42. Litvan I, Goldman JG, Troster Al, et al. Diagnostic criteria for mild cognitive impairment in Parkinson's disease: Movement Disorder Society Task Force guidelines. Mov Disord 2012;27(3):349356.

43. Borkovec TD, Sibrava NJ. Problems with the use of placebo conditions in psychotherapy research, suggested alternatives, and some strategies for the pursuit of the placebo phenomenon. $J$ Clin Psychol 2005;61(7):805-818.

44. Hamilton M. The assessment of anxiety states by rating. Br J Med Psychol 1959;32(1):50-55. 
45. Jenkinson C, Fitzpatrick R, Peto V, Greenhall R, Hyman N. The PDQ-8: Development and validation of a short-form Parkinson's Disease Questionnaire. Psychol Health 1997;12(6):805-814.

46. Herdman M, Gudex C, Lloyd A, et al. Development and preliminary testing of the new five-level version of EQ-5D (EQ-5D-5L). Qual Life Res 2011;20(10):1727-1736.

47. Coast J, Peters TJ, Natarajan L, Sproston K, Flynn T. An assessment of the construct validity of the descriptive system for the ICECAP capability measure for older people. Qual Life Res 2008;17(7):967-976.

48. Wimo A, Gustavsson A, Jonsson L, Winblad B, Hsu MA, Gannon B. Application of Resource Utilization in Dementia (RUD) instrument in a global setting. Alzheimers Dement 2013;9(4):429-435 e417.

49. Liebowitz MR. Social phobia. Mod Probl Pharmacopsychiatry 1987;22:141-173.

50. Sheehan DV, Lecrubier $\mathrm{Y}$, Sheehan $\mathrm{KH}$, et al. The Mini-International Neuropsychiatric Interview (M.I.N.I.): the development and validation of a structured diagnostic psychiatric interview for DSM-IV and ICD-10. J Clin Psychiatry 1998;59 Suppl 20:22-33;quiz 34-57.

51. Hamilton M. A rating scale for depression. J Neurol Neurosurg Psychiatry 1960;23:56-62.

52. Sockeel P, Dujardin K, Devos D, Deneve C, Destee A, Defebvre L. The Lille apathy rating scale (LARS), a new instrument for detecting and quantifying apathy: validation in Parkinson's disease. J Neurol Neurosurg Psychiatry 2006;77(5):579-584.

53. Trenkwalder C, Kohnen R, Hogl B, et al. Parkinson's disease sleep scale--validation of the revised version PDSS-2. Mov Disord 2011;26(4):644-652.

54. Carver CS. You want to measure coping but your protocol's too long: consider the brief COPE. Int J Behav Med 1997;4(1):92-100.

55. Wells A, Davies MI. The Thought Control Questionnaire - a Measure of Individual-Differences in the Control of Unwanted Thoughts. Behav Res Ther 1994;32(8):871-878.

56. Goetz CG, Tilley BC, Shaftman SR, et al. Movement Disorder Society-sponsored revision of the Unified Parkinson's Disease Rating Scale (MDS-UPDRS): scale presentation and clinimetric testing results. Mov Disord 2008;23(15):2129-2170.

57. Zarit SH, Reever KE, Bach-Peterson J. Relatives of the impaired elderly: correlates of feelings of burden. Gerontologist 1980;20(6):649-655.

58. Yang XY, Sun J, Luo J, et al. Regional homogeneity of spontaneous brain activity in adult patients with obsessive-compulsive disorder before and after cognitive behavioural therapy. $\mathrm{J}$ Affect Disord 2015;188:243-251.

59. Yang X-y, Luo J, Liu J, et al. Effects of cognitive behavioral therapy on white matter fibers of patients with obsessive-compulsive disorder as assessed by diffusion tensor imaging: study protocol for a parallel group, controlled trial. Asia Pacific Journal of Clinical Trials: Nervous System Diseases 2016;1(3):116.

60. Tomlinson CL, Stowe R, Patel S, Rick C, Gray R, Clarke CE. Systematic Review of Levodopa Dose Equivalency Reporting in Parkinson's Disease. Movement Disord 2010;25(15):2649-2653.

61. Lopes R, Delmaire C, Defebvre L, et al. Cognitive phenotypes in parkinson's disease differ in terms of brain-network organization and connectivity. Hum Brain Mapp 2017;38(3):1604-1621.

62. Galantucci S, Agosta F, Stefanova E, et al. Structural Brain Connectome and Cognitive Impairment in Parkinson Disease. Radiology 2017;283(2):515-525.

63. White IR, Royston P, Wood AM. Multiple imputation using chained equations: Issues and guidance for practice. Stat Med 2011;30(4):377-399.

64. Groenwold RHH, Donders ART, Roes KCB, Harrell FE, Moons KGM. Dealing With Missing Outcome Data in Randomized Trials and Observational Studies. Am J Epidemiol 2012;175(3):210-217. 65. Royston P. Multiple imputation of missing values: update. Stata J 2005;5(2):188-201.

66. Goldsmith LP, Dunn G, Bentall RP, Lewis SW, Wearden AJ. Therapist Effects and the Impact of Early Therapeutic Alliance on Symptomatic Outcome in Chronic Fatigue Syndrome. PLoS One 2015;10(12):e0144623.

67. Crits-Christoph P, Mintz J. Implications of therapist effects for the design and analysis of comparative studies of psychotherapies. J Consult Clin Psychol 1991;59(1):20-26. 
CHAPTER 8 | CBT FOR ANXIETY IN PD: RCT

68. Crits-Chrisoph P, Baranackie K, Kurcias J, et al. Meta-Analysis of Therapist Effects in Psychotherapy Outcome Studies. Psychotherapy Research 1991;1(2):81-91. 



\section{9}

Cognitive behavioural therapy for anxiety disorders in Parkinson's disease. development and content of a sregralised and tailored treatment pro ran me

Anne Mulders, Albert Leentjens, Kathy Dujar n, Bémngere Flinois, Anne-Claire Leterme, Annelien Duits, Marjolein de Vugt, (ark Kui , Anja Moonen (submitted)

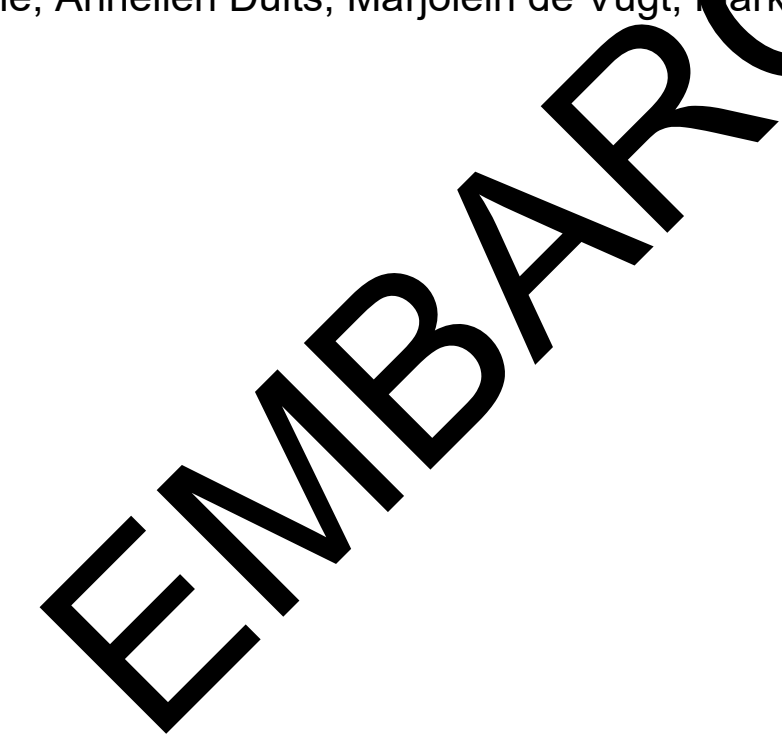




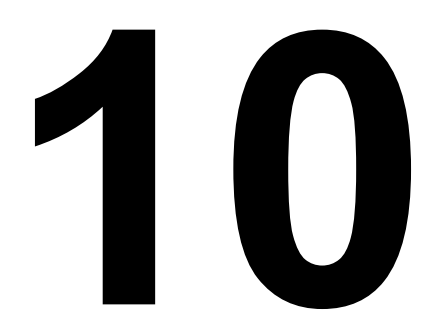

General Discussion 
In recent years, advances in both neuroimaging and electrophysiological techniques, as well as clinical experience, have resulted in new insights into the neural mechanisms underlying neurological and psychiatric disorders. Many findings have indicated associations between a dysfunction of cortico-basal ganglia-thalamocortical circuits and several motor, psychiatric, and cognitive symptoms that are often comorbid and trans diagnostic. The cortico-basal ganglia-thalamocortical circuits involve a series of parallel, partially segregated and integrated loops, including the motor circuits, involved in control of motor programmes, the dorsolateral prefrontal circuit, involved in associative and cognitive functions, and the orbitofrontal and anterior cingulate circuits, involved in limbic control and motivated behaviour ${ }^{1,2}$. In this thesis, we studied two disorders that are linked to alterations in cortico-basal ganglia-thalamocortical circuits: Parkinson's disease (PD) and obsessive-compulsive disorder (OCD).

PD and OCD have several theoretical and clinical similarities. First of all, deep brain stimulation of the subthalamic nucleus (STN-DBS) is a neurosurgical treatment for both $\mathrm{PD}$ and $\mathrm{OCD}$. Second, obsessive symptoms and traits are not uncommon in $\mathrm{PD}^{3}$, and patients with a longer disease duration have been found to have more severe obsessive-compulsive symptoms ${ }^{3}, 4$. Third, both PD and OCD are associated with cognitive rigidity, referring to the tendency to develop and perseverate in particular cognitive or behavioural patterns, even when, based on task-relevant information in the environment, that pattern is no longer effective ${ }^{5,6}$. The cortico-basal gangliathalamocortical circuits are believed to constitute the neural substrate of intact cognitive flexibility ${ }^{7}$. Fourth, at the phenotype level, both PD and OCD may be characterised by trans diagnostic mood symptoms, such as anxiety. A challenge is to find common and distinct neurobiological correlates of neuropsychiatric symptoms that are related to alterations to the above-mentioned circuits and to improve our understanding of the underlying neural mechanisms. DBS studies may help in studying dysfunctional brain circuits and their relation to the occurrence of emotional and cognitive symptoms. The first part of this thesis was aimed at increasing our understanding of the role of the STN in OCD symptomatology (Chapter 2 and $\mathbf{3}$ ), and to study underlying mechanisms of emotional and cognitive side-effects following STNDBS in PD (Chapter $\mathbf{4}$ and $\mathbf{5}$ ) based on observations from DBS studies. 
The second part of this thesis focused on mood symptoms in PD. Mood symptoms, such as anxiety, can be very distressing to patients and may worsen motor symptoms or be perceived as more disabling than motor symptoms. Both mood symptoms and motor symptoms can fluctuate over the course of the day and are difficult to measure with routine cross-sectional assessments that are used in movement disorder clinics. Therefore, not much is known about the relationship between mood and motor fluctuations ${ }^{8}$, which makes treatment of these symptoms challenging. Moreover, there is currently no evidence-based treatment available to treat anxiety in PD. We aimed to explore the usability of the experience sampling method (ESM) in PD in studying associations between motor and mood symptoms in patients who suffer from motor fluctuations (Chapter 6 and 7). Finally, we aimed to develop a treatment module for anxiety in PD that is supported by the knowledge of the underlying aetiology and correlations of anxiety in PD (Chapter 8 and 9). The current chapter discusses the interpretation of the main findings, their scientific and clinical implications, as well as methodological considerations.

\section{Main findings part 1: DBS studies in OCD and PD}

The role of the STN in OCD circuitry

In chapter 2 we performed a review of the functional role of the STN in the pathophysiology of OCD by integrating electrophysiological findings from STN-DBS studies in OCD as well as neuroanatomical information. Although literature on the role of the STN in OCD circuitry and its pathophysiological mechanisms is limited, recent findings are indicative of an electrophysiological dysfunction in the associative and limbic (i.e. non-motor) parts of the STN ${ }^{9}$. Compared to PD patients, STN neurons in OCD exhibit a lower firing rate, less frequent but longer bursts, increased burst activity in the anterior ventromedial area, an asymmetrical left-sided burst distribution, and a predominant oscillatory activity in the $\delta$-band ${ }^{9},{ }^{10}$. Moreover, there is direct evidence for the involvement of the STN in both checking behaviour and OCD symptoms, which are both related to changes in electrophysiological activity in the non-motor STN ${ }^{9}, 11$. STN dysfunction seems to cause a disruption of information processing of associative and limbic processes, hence leading to obsessions and compulsions ${ }^{10}$ 11. These findings are in line with proposed models of decision-making and action-selection. These models suggest a key role for the STN through its central position within the corticobasal ganglia-thalamocortical circuits on one hand, and because of the integration of 
multiple streams of information from the environment believed necessary for establishing priorities for action-planning and potential behaviour, as well as for the coordination of action, stream of thoughts and motivation ${ }^{12}$. We believe that through a combination of mechanisms, STN-DBS interrupts the disturbed information-processing that seems responsible for the pathological repetitive behaviours characteristics for OCD by restoring the imbalance between direct and indirect pathways, resulting in normalisation of connectivity within the cortico-basal ganglia-thalamocortical circuits, and consequently to a reduction in symptoms ${ }^{13}$.

While the above findings suggest the STN to be a promising mechanism-based target for OCD, in Chapter 3 we present a case in which DBS programming of the STN turned out to be challenging. A patient with a 30 -year history of treatment-refractory OCD underwent anteromedial STN-DBS. Despite a clear mood-enhancing effect, stimulation at voltages greater than $1.5 \mathrm{~V}$ caused severe and persistent motor sideeffects that resembled chorea and ballism. The aim of DBS is to tailor treatment on a patient-specific basis to deliver optimal therapeutic effects while avoiding stimulationassociated side effects ${ }^{14,15}$. While the underlying mechanisms and pathophysiology of DBS-induced motor side-effects are not well understood, we have listed several potential causes for the observed side-effects. Firstly, it is possible that STN-DBS resulted in an increase in striatal dopamine release and, subsequently, in hyperdopaminergic behaviours, such as dyskinesia. Second, due to individual differences in the structural and functional anatomy of STN subdivisions, it is possible that, although the anteromedial part of the STN was targeted, this part did not constitute the non-motor portion of the STN in our patient. Consequently, motor sideeffects may have developed either directly by stimulation of the motor portion of the STN or indirectly by current spreading of contact points in close proximity to the motor portion of the STN. Because in the present case the side-effects made optimal DBS programming impossible, the patient underwent stereotactic reimplantation of the electrodes at the Ventral Capsule / Ventral Striatum (VC/VS) region, with the deepest electrode contact point in the area of the Nucleus Accumbens (NAc), which resulted in a reduction of $50 \%$ in OCD symptoms two years following surgery. 
Underlying mechanisms of emotional and cognitive side-effects of STN-DBS in PD patients

Despite optimal improvement in motor functioning following STN-DBS in PD patients, there have been reports on the occurrence of neuropsychiatric, behavioural and cognitive changes after surgery. The underlying mechanisms contributing to changes in mood, behaviour and cognition following STN-DBS in PD are not well understood. Experimental studies have found substantial disturbance and inhibition of 5-HT neurotransmission during STN-DBS ${ }^{16,17}$ and evidence for a STN- lateral habenula dorsal raphe nucleus network, which modulates $5-\mathrm{HT}$ function and behaviour that reflect key symptoms of depression ${ }^{18}$. Moreover, depression has generally been associated with a dysfunction of the 5-HT neurotransmitter system. Because depression is more frequent in PD patients compared to the general population ${ }^{19}$, and $\mathrm{PD}$ has been associated with changes in $5-\mathrm{HT}^{20}$, we hypothesised that PD patients have a 5-HT vulnerability that, in combination with a decrease in 5-HT transmission elicited by STN stimulation, may increase susceptibility to develop depression and other psychiatric problems following STN-DBS.

In Chapter 4 we aimed to study the role of 5-HT on mood and behaviour in PD patients with STN-DBS in a double-blind, placebo-controlled crossover study by temporarily reducing the level of available $5-\mathrm{HT}$ in the brain by means of acute tryptophan depletion (ATD). Participants were tested in DBS-ON and DBS-OFF condition. We hypothesised that ATD in combination with STN-DBS (DBS-ON) might lead to lower mood, and other behavioural changes such as reduced inhibitory control and deficits in emotional processing. Due to lagging inclusion, the study was terminated after including 7 patients. In these 7 patients, we found that $5-\mathrm{HT}$ manipulation by ATD did not lead to dissociable effects on mood, emotional processing and inhibitory control. In fact, for the main outcome measure mood, we observed effects in opposite direction of what was hypothesised. Instead of a decrease in total mood disturbance, we found an increase in total mood disturbance in DBS-OFF condition. The return of motor symptoms as a result of switching the stimulator off turned out to be emotionally overwhelming for participants, which in turn masked possible subtle differential effects that ATD might have had on mood. Overall, apart from recruitment difficulties, we concluded that the study was not feasible due to the great patient burden of stopping the DBS stimulation, and that continuation would be ethically unacceptable, as any 
further results that might have been found would not be in proportion to the difficulties and risks imposed on the research participants. While the findings of the study suggest that psychiatric side-effects following STN-DBS in PD patients cannot be directly linked to a reduction in 5-HT activity, these results should be interpreted with caution due to the small sample size.

Besides mood, emotional processing and inhibitory control, we aimed to study underlying mechanisms of changes in cognition following STN-DBS in PD. Research on the factors contributing to cognitive changes following surgery have led to inconsistent findings. It has been proposed that surgical factors, such as cortical and subcortical lesions due to the lead trajectory, may contribute to cognitive changes following STN-DBS. In Chapter 5 we studied whether characteristics of the surgical trajectory, including the number of microelectrode recording (MER) trajectories, the STN length as measured by MER, and cortical entry points (sagittal and coronal angles) were associated with cognitive decline one year after surgery. We found a significant decline in a number of tests for executive function one year postoperatively, including a decline in verbal fluency, performance on the Stroop Colour-Word test and the Trail Making Test-B (TMT-B). Changes in TMT-B were associated with the coronal entry point in the right hemisphere. However, only 5 patients showed clinically relevant cognitive decline in TMT-B performance. No associations were found between the STN length or the number of MER passes and changes in neuropsychological functioning. This suggests that, from a cognitive point of view, the use of MER does not seem to impose an increased risk of decline one year following surgery. Whereas the electrode passage through the right prefrontal lobe may contribute to subtle changes in executive function, only few patients showed clinically relevant cognitive decline.

\section{Scientific and clinical implications part 1}

The development of DBS has opened new opportunities to access and interrogate dysfunctional brain circuits. Recording the electrical activity of the STN during DBS, testing its therapeutic potential of regulating the behavioural outcome of malfunctioning circuits, but also the occurrence of (unexpected) mood, cognitive and motor outcomes, have contributed to a better understanding of the functional role of the STN in the context of PD and OCD. 
The findings from the OCD studies are consistent with the idea that OCD can be regarded a 'circuit' disorder. The observation that DBS of different structures along the proposed dysfunctional circuit (STN and VC/VS) can be effective in reducing obsessive and compulsive symptoms in the same patient seem to confirm this, and is consistent with findings of similar percentages of improvement following DBS in different target areas $^{14,15}$. Moreover, it is in line with theories suggesting that the mechanisms of DBS can be viewed from a network perspective, where DBS has effects on the target structure itself, but also on distal structures through disruption of disturbed informationprocessing within the network ${ }^{21}$. Recently, new studies have emerged that reported changes in neurophysiological activity throughout the $\mathrm{VS}^{22}$ and $\mathrm{STN}^{23}$ following provocation of obsessions. In the latter study, the ventro-medial STN of OCD patients showed distinct theta oscillatory activity that decreased following provocation of OCD symptoms and was inversely correlated with symptom severity over time ${ }^{23}$. Identifying regions of distinct, and possibly unique, activity related to symptoms contributes not only to an increased understanding of the neural pathways involved in symptoms, but might also be used by neurosurgeons to ensure accurate surgical placement of the leads and careful programming to achieve maximal therapeutic effect. Ideally, with increased knowledge on the different target structures, their clinical efficacy, as well as adverse side-effects, clinicians may choose between several target areas in the future, depending on the main symptom targeted and the risk profile of the patient. Moreover, an individualised, patient-specific selection method based on, for example, preoperative diffusion-weighted imaging and tractography and functional MRI may improve DBS targeting.

Concerning the DBS studies in PD, we did not find evidence for a role of the 5-HT neurotransmitter system in the occurrence of psychiatric side-effects following STNDBS as was reported by experimental studies ${ }^{16,17,24}$. While the findings from our study should be interpreted with caution due to methodological issues, it is also possible that the preclinical model used in experimental studies may not be an ideal model to study human PD and changes in 5-HT neurotransmission. There is evidence of a different neurophysiology of the 5-HT receptor system in animals ${ }^{25}$. Moreover, while most preclinical models use acute dopamine lesion models, the degenerative process in humans is already present years before the first symptoms emerge, which might lead to adaptive processes that are not present in animals ${ }^{25,26}$. Studying whether STN-DBS 
results in a decrease in 5-HT in PD patients, e.g. by studying 5-HT in cerebrospinal fluid before and after surgery, might be a valuable intermediate translational step when studying the effect of STN-DBS on 5-HT and related behaviour.

Consistent with previous studies we found significant changes in executive function tests one year following surgery. Except for an association between the right coronal angle and changes in TMT-B, we found no associations between the number of MER trajectories used during surgery and the STN length as measured by MER. This finding is important for neurosurgeons, as it indicates that they do not have to be restrained by fear of cognitive side-effects when attempting to have more possibilities for postoperative stimulation parameters adjustments by the use of (multiple) MER. The angle of approach, however, might contribute to subtle dysfunction. While we cannot draw any conclusions about causality, and changes in cognition following STN-DBS are likely to be multifactorial, this finding warrants further research. Preferably, all factors contributing to cognitive decline following surgery, including patient inherent characteristics, as well as stimulation and surgical characteristics, should be integrated in future studies. Machine learning might be a promising tool to investigate the complex interplay between these factors in predicting which patients are at risk to show cognitive decline following STN-DBS. However, large sample sizes are required and while data sharing and multicentre studies could improve power, the interpretation of findings could be complicated by different neurosurgical approaches between centres to target the STN. Importantly, changes in cognitive decline were based on neuropsychological test performance and do not necessarily translate into impairment in daily activities. Only few patients showed clinically relevant decline following surgery, which seems to indicate that from a cognitive point of view, DBS is a relatively safe procedure.

\section{Main findings part 2: mood symptoms in PD}

The usability of ESM in PD

Up to $50 \%$ of PD patients develop motor fluctuations after long-term treatment with levodopa. Such fluctuations in motor symptoms are often accompanied by distressing mood fluctuations, such as fluctuations in anxiety and depressive symptoms ${ }^{8}, 27$. Research involving mood fluctuations, their underlying mechanisms, and their 
relationship with motor fluctuations has so far led to inconsistent results ${ }^{8}$. Chapter 6 aimed to explore whether it is possible to identify longitudinal associations between motor symptoms and mood states in a PD patient with motor fluctuations using ESM, and to generate a symptom network of these associations. ESM includes multiple short, repeated, within-subject assessments at random moments during the day that can include motor symptoms, mood states and contextual factors. In an $n=1$ study, a 53-year old PD patient collected ESM data for 34 consecutive days. A set of dependent variables included several motor symptoms, on/off state, and the mood variables anxiety, cheerful and down. Network analyses revealed moment-to-moment variability and prospective associations between motor and mood states. The variables anxiety and cheerful appeared to play a more important role than the variable down, because they had stronger associations with several motor symptoms, including tremor and rigidity. These results suggest that affect plays an important role in the way motor symptoms are perceived.

In routine patient care much briefer ESM sampling periods are commonly used, since longer periods are considered burdensome for patients. In Chapter 7 we aimed to explore the usability of routinely collected ESM data over a brief period of time in PD patients, and to explore the sensitivity to detect associations between mood states, contextual factors, motor symptoms and motor state on both a group and individual level. Eleven PD patients with motor fluctuations collected ESM data for five consecutive days. On a group level, ESM was able to identify clear associations between affective state, contextual factors and motor symptoms. Regardless of whether a patient was in an 'on' or 'off' state, higher scores on positive affect were associated with lower self-perceived severity of tremor, rigidity, balance problems and walking problems. Negative affect was not associated with any of the motor symptoms. These findings are in line with the results from the network analysis described in chapter 6 , in which feeling down did not influence motor symptom severity, while cheerfulness was significantly associated with lower scores for tremor and rigidity. Although we were able to display moment-to-moment fluctuations in mood and motor symptoms in individual patients, analyses on an individual level revealed much less significant associations that mostly, but not always, were in line with the group level analyses. We think the most likely explanation for the failure to identify significant associations between symptoms in individual patients is lack of power. 


\section{Treatment of anxiety in $P D$}

Anxiety is a common problem in PD and results in reduced quality of life. At present, there is no evidence-based treatment available for anxiety in PD. Anxiety in PD is often the result of a complex interaction between physiological and psychological factors. Given this complexity, psychotherapeutic treatment of anxiety requires adaptations tailored to the specific needs of PD patients and supported by knowledge of the underlying aetiology of $\mathrm{PD}^{28}$. Chapter 8 presents the rationale and methodology of a two-centre randomised controlled trial (RCT) aimed at studying the clinical effectiveness, cerebral correlates, and cost-effectiveness of a specialised cognitivebehavioural therapy (CBT) programme for anxiety in PD. PD patients with anxiety and their caregivers (optional) were randomised to $\mathrm{CBT}$ and clinical monitoring (intervention group) or clinical monitoring only (control group). All participants underwent standardised clinical, cognitive and behavioural assessment at baseline (t0), at the end of the intervention (t1) as well as 3 months after the intervention (t2). Moreover, participants randomised to the intervention group received an additional full assessment at 6-months follow-up (t3). At t0 and $\mathrm{t} 1$ participants underwent Magnetic Resonance Imaging scanning. The duration of the intervention was 10 to 12 weeks.

Chapter 9 reports the development and content of the CBT programme. Focus group interviews were used to explore the needs and wishes of PD patients (and their caregivers) who suffer from anxiety with respect to the treatment of anxiety. During these focus group interviews, the following topics were discussed: (1) former experiences with CBT or other psychotherapeutic interventions; (2) content of the therapy sessions and relevant themes; (3) structure of the therapy sessions, including the number of sessions and the duration of each session; (4) home assignments; (5) potential (practical) barriers of undergoing CBT, and (6) caregiver participation. Based on the focus group interviews we made several adjustments. The final programme addresses (but is not limited to) anxiety symptoms that can be considered more or less specific for Parkinson patients, such as 'wearing off' anxiety, fear of falling or feeling judged by others in social situations. The CBT programme provides a range of cognitive and behavioural skills, including psycho-education, relaxation techniques, cognitive restructuring, problem solving, exposure, personal habits and selfmanagement, that can help patients in the process of adjusting to current and future impairments and changes, and empower patients to build confidence in their capacity 
to deal with them. We developed a workbook for patients and a treatment manual for therapists, in which possible challenges during treatment are discussed, such as the uncertainty about disease progression, cognitive deficits, and barriers to continue the therapy programme and do the home assignments.

Recently, we completed the final follow-up assessment of the RCT. In total, we included 48 PD patients. Of these, 24 were randomised to the intervention group and 24 to the control group. Two patients, of which one was randomised to the intervention group and one to the control group, dropped out of the study during the intervention period. In between the first (t1) and second (t2) follow-up assessments, two more patients dropped out of the study, again one randomised to the intervention group and one patient randomised to the control group. Thus, a total of 46 patients completed the first follow-up and 44 patients completed the RCT. We are currently analysing the clinical and cost-effectiveness of the study, as well as changes in cerebral connectivity following CBT.

\section{Scientific and clinical implications part 2}

ESM allows for a more naturalistic and reliable assessment of fluctuating symptoms which may help in achieving optimal symptom control. While we were able to reveal statistically significant prospective associations when ESM data was collected for a longer period of time (chapter 6), ESM data collection as used in chapter 7 was apparently not sensitive enough to detect within-person associations. The sensitivity may be increased by collecting data over a longer period of time as shown in the $n=1$ study, by increasing the compliance or by making the answers more sensitive to change (e.g. by increasing the number of potential scores on the Likert item or by using visual analogue scale scores). The ultimate goal is to use ESM in a non-burdening way in routine patient care as a tool to facilitate patient self-monitoring and as a guidance for clinicians for (personalised) treatment. There is an urgent need for harmonisation, validation and optimisation of this technique, since ESM will likely have a more important role in future studies as well as in clinical practice. ESM data may serve, for example, as a parallel ground truth to objective data, such as obtained by monitoring motor symptoms by wearable sensors ${ }^{29}$, since these alone may not be representative for the disease burden or experienced disability. In addition, combining objective and subjective data on motor and non-motor symptoms can potentially be used as input 
signal for adaptive (or closed-loop) DBS ${ }^{29}$. Adaptive DBS aims to automatically adapt stimulation parameters to the fluctuating clinical state of the patient, where stimulation is only provided when necessary ${ }^{30}$. The idea is that adaptive DBS results in fewer stimulation-induced side-effects compared to conventional DBS.

Finally, both ESM studies highlight the role of positive affect on self-perceived severity of motor symptoms. Since most studies in PD have focused on the role of negative mood states in relation to motor symptoms, we believe that it is necessary to include positive affect in the design of future studies. Moreover, enhancing the frequency and persistence of positive affect in daily life might be a promising adjunctive nonpharmacological target for reducing the burden of motor fluctuations and increasing quality of life.

Interestingly, besides reductions in negative affect, CBT for anxiety has been associated with improvements in positive affect ${ }^{31}$. While we are still awaiting the final results of the RCT investigating the effects of $C B T$ for anxiety in PD, this study has, if proven effective, great relevance for clinical practice. It provides PD patients with behavioural and anxiety management techniques that may help them in dealing with current anxiety or new (disease-related) problems that can cause anxiety in the future. In addition, it may give lasting benefits on well-being, quality of life, and possibly on motor symptoms. It would be the first evidence-based treatment available for anxiety in PD. The manual for therapist describes the structure and content of the therapy in detail, and addresses several PD specific challenges that may occur during the course of CBT. Besides clinical and cost effectiveness, future studies could study the impact of CBT on different types of symptoms of anxiety.

\section{METHODOLOGICAL CONSIDERATIONS}

Methodological considerations, as well as strengths and limitations of the research presented in this thesis will be discussed in the following section.

\section{Study designs}

Multiple study designs were used in the studies presented in this thesis. The review in chapter 2 that described and discussed the state of the science regarding neuroanatomical and electrophysiological findings from DBS studies regarding the 
functional role of the STN in relation to OCD symptomatology, is a narrative review. Selection bias may occur since it does not use a predetermined structured method to search, select, appraise and summarise study findings, as is being done in a systematic review ${ }^{32}$. For that reason, systematic reviews are often favoured over narrative reviews. One of the reasons for performing a narrative review rather than a systematic review was the recent publication of a systematic review and meta-analysis on DBS in different target areas for $O C D^{15}$. While the systematic review provided valuable information of the treatments effects of DBS in OCD, it does not contribute to the understanding of how stimulation of a specific target area results in a reduction in OCD symptoms, and the involvement of that area in the pathophysiology of the disorder. The strength of our review is that it contributed to an increased understanding of the functional role of the STN in OCD symptomatology from a theoretical and contextual point of view. From this perspective, we believe that our review served a different purpose and should be seen as complementary to the available systematic reviews.

In chapter 4 a randomised placebo-controlled, cross-over design was used, in which both the participant and researcher were blinded for the intervention (ATD or placebo mixture). Patients were tested in four different conditions: placebo + DBS-ON, Placebo + DBS-OFF, TRP depleted + DBS-ON and TRP depleted + DBS-OFF. Crossover designs require fewer patients to be included due to increased power. Moreover, a wash-out period controlled for carry-over effects. However, a limitation of the design was that it required patients to be available for two full days. Moreover, due to the vulnerability of the research population we used strict in- and exclusion criteria. We experienced recruitment difficulties due to these requirements, which might have induced selection bias. Moreover, we underestimated the emotional impact of turning the stimulator off which not only influenced our results, but made participating in the study increasingly burdensome for patients. We had to conclude that the study was not feasible and continuation was ethically debatable. Turning the stimulator off is not uncommon for scientific purposes. Based on our findings, we argue that the emotional impact of being confronted with motor symptoms after turning the stimulator off should always be taken into account when designing a study and when interpreting its results. 
The retrospective study described in chapter 5 allowed to examine data from routine clinical care. Unfortunately, due to uncontrollable factors such as differences in followup time (e.g. neuropsychological assessment was performed at 18 months instead of 12 months following surgery), many participants had to be excluded, resulting in a smaller sample size and, subsequently, reduced power.

In chapter 6 we used an $n=1$ design of ESM data collected for a longer period of time (34 days), and in chapter 7 we used group vs. individual analyses of ESM data collected for a brief period of time (5 days). Group analyses may reveal population based patterns that may lead to more general treatment or research recommendations, such as studying the role of positive affect on the way motor symptoms are perceived, while individual analyses can be used to establish a personalised treatment plan ${ }^{33}$. While the results from the $n=1$ study and individual analyses cannot be generalised, they are very relevant for clinical practice. Using different study designs provided valuable information of the potentials of ESM in clinical practice, as well as flaws that still require improvement.

In chapter 8 we describe the rationale and methodology of a two-centre RCT to test the clinical effectiveness of the treatment programme we developed for anxiety in PD. RCTs are considered the gold standard for providing convincing evidence. However, dropout in longitudinal RCTs is common and might threaten the validity of the results, especially when dropout rates are different between study groups. Possibly, patients assigned to the control group might drop out because they want/require immediate treatment for their anxiety symptoms. To prevent dropout, and because we found it ethically not favourable to have patients wait for a period longer than 6 months after the start of the study, we decided to have the last follow-up assessment 3 months following the end of the intervention. Since we are aware that 3 months follow-up time is short to draw conclusions regarding the long-term effectiveness of the treatment, we included a 6 months within-subject follow-up assessment for the patients who were randomised to the intervention group.

\section{Approaches to data-analysis}

All statistical approaches were carefully chosen in line with the research question posed. Besides descriptive statistics, t-tests, Wilcoxon-rank order tests, multiple 
regression and repeated measures analysis of covariance (rm-ANCOVA), we also used more advanced statistical analyses, such as network analysis, multilevel linear regression analyses and permutation testing. An important limitation of the presented studies and data-analysis approaches is that we were not able to draw conclusions regarding causality. In chapter 4 , we used rm-ANCOVA. Given the small sample size, the results from this analysis should be interpreted with caution. In chapter 5, when comparing pre- and postoperative performance on neuropsychological tests, we did not correct for multiple comparison in order to reduce the risk of type II error given the importance of detecting any adverse effects of surgery. In chapter 6 and 7 we added an extra variable to control for time trends to all regression models. Given the exploratory nature of the study described in chapter 6 , we did not control for multiple comparisons. In chapter 7 , we experienced convergence issues after running the regression models. Moreover, the assumption for normality was not met for all variables. To overcome these limitations and obtain valid and interpretable results, we performed permutation testing.

\section{Interventions and measurement instruments}

The study in chapter 4 used the acute tryptophan depletion (ATD) paradigm to study the effects of a temporarily reduction of the level of available serotonin (5-HT) in the brain on mood, emotional processing and inhibitory control. While the ATD paradigm is a popular method to study the effects of low $5-\mathrm{HT}$ in humans, there is some controversy about the extent to which ATD alters 5-HT function (see ${ }^{34}$ for a review on ATD in humans). One of the most important limitations of ATD is that 5-HT depletion cannot be localised to particular regions in the brain ${ }^{34}$, which might not only impact the effect of ATD on different outcome parameters, but makes it difficult to mimic STNDBS induced 5-HT changes as observed in experimental studies. Moreover, studies have found that in many circumstances, ATD does not lower mood and the mechanisms of these differential effects are currently unknown ${ }^{34}$. It means that the results from a tryptophan depletion study should be interpret with caution.

In chapter 6 and 7 we used ESM to study associations between mood and motor symptoms and contextual factors in PD. ESM allowed us to measure the subjectively perceived severity of fluctuating mood and motor symptoms, which are usually not captured by routine cross-sectional assessments that might cause response bias. 
Combining ESM with wearable sensor data to objectify the motor symptoms could improve the validity of the method. Moreover, in both studies we experienced that assessments in off-state were limited, which reduced the power when analysing the modifying role of motor state on the associations between motor and mood states. An alternative is to programme the assessments at fixed times or to ask the patient to only answer the questionnaire when being off. However, this prevents capturing the fluctuating nature of symptoms. Moreover, reactivity due to predictability may occur, which can induce behavioural changes ${ }^{33}$.

\section{CONCLUDING REMARKS}

With this thesis we provided small pieces of the puzzle regarding the functional role of the STN in the pathophysiology of OCD, and of factors that may (or may not) contribute to changes in mood and cognition following STN-DBS in PD patients. Moreover, we showed that ESM is a promising method to study associations between motor and mood symptoms in PD patients. We provided recommendations to improve the sensitivity of the method in order to be a potential instrument to use for personalising treatment in routine patient care. Finally, we developed a treatment programme for anxiety in PD, which is currently underdiagnosed and undertreated. We do not at this time have the final results of the RCT investigating the clinical and cost effectiveness of the programme, however, preliminary analysis (not included in this thesis) showed promising results. While more research is needed in the topics of this thesis, I believe that our findings contribute to improved patient care and eventually to an increase in quality of life of patients with OCD and PD. 


\section{REFERENCES}

1. Alexander GE, DeLong MR, Strick PL. Parallel organization of functionally segregated circuits linking basal ganglia and cortex. Annu Rev Neurosci 1986;9:357-381.

2. Haynes WI, Haber SN. The organization of prefrontal-subthalamic inputs in primates provides an anatomical substrate for both functional specificity and integration: implications for Basal Ganglia models and deep brain stimulation. J Neurosci 2013;33(11):4804-4814.

3. Alegret M, Junque C, Valldeoriola F, Vendrell P, Marti MJ, Tolosa E. Obsessive-compulsive symptoms in Parkinson's disease. J Neurol Neurosurg Psychiatry 2001;70(3):394-396.

4. Tomer R, Levin BE, Weiner WJ. Obsessive-Compulsive Symptoms and Motor Asymmetries in Parkinsons-Disease. Neuropsy Neuropsy Be 1993;6(1):26-30.

5. Morris L, Mansell W. A systematic review of the relationship between rigidity/flexibility and transdiagnostic cognitive and behavioral processes that maintain psychopathology. J Exp Psychopathol 2018;9(3).

6. Remijnse PL, van den Heuvel OA, Nielen MMA, et al. Cognitive Inflexibility in ObsessiveCompulsive Disorder and Major Depression Is Associated with Distinct Neural Correlates. Plos One 2013;8(4).

7. Gu BM, Park JY, Kang DH, et al. Neural correlates of cognitive inflexibility during task-switching in obsessive-compulsive disorder. Brain 2008;131:155-164.

8. van der Velden RMJ, Broen MPG, Kuijf ML, Leentjens AFG. Frequency of mood and anxiety fluctuations in Parkinson's disease patients with motor fluctuations: A systematic review. Mov Disord 2018;33(10):1521-1527.

9. Welter ML, Burbaud P, Fernandez-Vidal S, et al. Basal ganglia dysfunction in OCD: subthalamic neuronal activity correlates with symptoms severity and predicts high-frequency stimulation efficacy. TransI Psychiatry 2011;1:e5.

10. Piallat B, Polosan M, Fraix V, et al. Subthalamic neuronal firing in obsessive-compulsive disorder and Parkinson disease. Ann Neurol 2011;69(5):793-802.

11. Burbaud $\mathrm{P}$, Clair $\mathrm{AH}$, Langbour $\mathrm{N}$, et al. Neuronal activity correlated with checking behaviour in the subthalamic nucleus of patients with obsessive-compulsive disorder. Brain 2013;136(Pt 1):304-317. 12. Haynes WI, Millet B, Mallet L. [Obsessive-compulsive disorder, a new model of basal ganglia dysfunction? Elements from deep brain stimulation studies]. Rev Neurol (Paris) 2012;168(8-9):649-654. 13. Chiken S, Nambu A. Mechanism of Deep Brain Stimulation: Inhibition, Excitation, or Disruption? Neuroscientist 2016;22(3):313-322.

14. KohI S, Schonherr DM, Luigjes J, et al. Deep brain stimulation for treatment-refractory obsessive compulsive disorder: a systematic review. Bmc Psychiatry 2014;14.

15. Alonso P, Cuadras D, Gabriels L, et al. Deep Brain Stimulation for Obsessive-Compulsive Disorder: A Meta-Analysis of Treatment Outcome and Predictors of Response. Plos One 2015;10(7):e0133591.

16. Tan SK, Hartung H, Visser-Vandewalle V, Steinbusch HW, Temel Y, Sharp T. A combined in vivo neurochemical and electrophysiological analysis of the effect of high-frequency stimulation of the subthalamic nucleus on 5-HT transmission. Exp Neurol 2012;233(1):145-153.

17. Tan SK, Janssen ML, Jahanshahi $A$, et al. High frequency stimulation of the subthalamic nucleus increases c-fos immunoreactivity in the dorsal raphe nucleus and afferent brain regions. $J$ Psychiatr Res 2011;45(10):1307-1315.

18. Hartung H, Tan SK, Temel Y, Sharp T. High-frequency stimulation of the subthalamic nucleus modulates neuronal activity in the lateral habenula nucleus. Eur J Neurosci 2016;44(9):2698-2707.

19. Reijnders JS, Ehrt U, Weber WE, Aarsland D, Leentjens AF. A systematic review of prevalence studies of depression in Parkinson's disease. Mov Disord 2008;23(2):183-189; quiz 313.

20. Marsh L. Depression and Parkinson's disease: current knowledge. Curr Neurol Neurosci Rep 2013;13(12):409.

21. Mclntyre CC, Hahn PJ. Network perspectives on the mechanisms of deep brain stimulation. Neurobiol Dis 2010;38(3):329-337. 
22. Miller KJ, Prieto T, Williams NR, Halpern CH. Case Studies in Neuroscience: The electrophysiology of a human obsession in nucleus accumbens. J Neurophysiol 2019;121(6):23362340.

23. Rappel P, Marmor O, Bick AS, et al. Subthalamic theta activity: a novel human subcortical biomarker for obsessive compulsive disorder. Transl Psychiat 2018;8.

24. Temel Y, Boothman LJ, Blokland A, et al. Inhibition of 5-HT neuron activity and induction of depressive-like behavior by high-frequency stimulation of the subthalamic nucleus. Proc Natl Acad Sci U S A 2007;104(43):17087-17092.

25. Scholtissen B, Verhey FR, Steinbusch HW, Leentjens AF. Serotonergic mechanisms in Parkinson's disease: opposing results from preclinical and clinical data. J Neural Transm (Vienna) 2006;113(1):59-73.

26. Scholtissen B, Verhey FR, Adam JJ, Weber W, Leentjens AF. Challenging the serotonergic system in Parkinson disease patients: effects on cognition, mood, and motor performance. Clin Neuropharmacol 2006;29(5):276-285.

27. Witjas T, Kaphan E, Azulay JP, et al. Nonmotor fluctuations in Parkinson's disease: frequent and disabling. Neurology 2002;59(3):408-413.

28. Dissanayaka NNW, White E, O'Sullivan JD, et al. Characteristics and Treatment of Anxiety Disorders in Parkinson's Disease. Mov Disord Clin Pract 2015;2(2):155-162.

29. Heijmans M, Habets JGV, Herff C, et al. Monitoring Parkinson's disease symptoms during daily life: a feasibility study. NPJ Parkinsons Dis 2019;5:21.

30. Habets JGV, Heijmans M, Kuijf ML, Janssen MLF, Temel Y, Kubben PL. An update on adaptive deep brain stimulation in Parkinson's disease. Mov Disord 2018;33(12):1834-1843.

31. Tirpak JW, Cassiello-Robbins C, Ametaj A, et al. Changes in positive affect in cognitivebehavioral treatment of anxiety disorders. Gen Hosp Psychiat 2019;61:111-115.

32. Greenhalgh T, Thorne S, Malterud K. Time to challenge the spurious hierarchy of systematic over narrative reviews? Eur J Clin Invest 2018;48(6):e12931.

33. Verhagen SJW, Berben JA, Leue C, et al. Demonstrating the reliability of transdiagnostic mHealth Routine Outcome Monitoring in mental health services using experience sampling technology. Plos One 2017;12(10):e0186294.

34. Young SN. Acute tryptophan depletion in humans: a review of theoretical, practical and ethical aspects. J Psychiatr Neurosci 2013;38(5):294-305. 

Summary - Samenvatting 
Neuropsychiatric symptoms are associated with a dysfunction of cortico-basal gangliathalamocortical circuits and include a broad range of psychiatric symptoms, such as depressive mood, euphoria, agitation and aggression, anxiety, sleep disturbance and disinhibition, and frequently occur in neurological, neurodegenerative, and psychiatric disorders. The basal ganglia are a group of interconnected sub-cortical nuclei and consist of the caudate nucleus, putamen, pallidal complex, nucleus accumbens, substantia nigra (pars compacta and reticulate) and the subthalamic nucleus (STN). In this thesis, we studied two disorders that are linked to alterations in cortico-basal ganglia-thalamocortical circuits: Parkinson's disease (PD) and obsessive-compulsive disorder (OCD).

$P D$ is a common neurodegenerative disorder with an estimated prevalence of $1-2 \%$ of the population of 65 years and older. Apart from the classical motor symptoms, such as resting tremor, rigidity, bradykinesia, and postural instability, many PD patients suffer from neuropsychiatric symptoms such as depression and anxiety. OCD is a neuropsychiatric disorder characterised by chronic intrusive and distressing thoughts or impulses (obsessions) and repetitive and ritualistic thoughts or mental acts (compulsions). Deep brain stimulation of the STN (STN-DBS) is a neurosurgical treatment for both PD and OCD. The first part of this thesis was aimed at increasing our understanding of the role of the STN in OCD symptomatology based on observations from DBS studies, and to study underlying mechanisms of emotional and cognitive side-effects following STN-DBS in PD. The second part of this thesis focused on mood symptoms in PD and aimed at studying the relationship between mood and motor symptoms in $\mathrm{PD}$, and to develop a psychotherapeutic treatment programme for anxiety in PD.

Chapter $\mathbf{1}$ is a general introduction into the theme and aims of this thesis. It provides information about PD and OCD, (neuropsychiatric) symptoms, treatment including DBS, as well as the research questions and outline of this thesis.

\section{Part 1}

Chapter 2 provides a narrative review on the functional role of the STN in OCD. By reviewing the literature of the last years, we believe that through its central position within the cortico-basal ganglia-thalamocortical circuits, the STN has a coordinating 
role in decision-making and action-selection mechanisms. Dysfunctional informationprocessing at the level of the STN is responsible for some of the core symptoms of OCD. Research confirms an electrophysiological dysfunction in the associative and limbic (non-motor) parts of the STN. Moreover, there is evidence for the involvement of the STN in both checking behaviour and OCD symptoms, which are both related to changes in electrophysiological activity in the non-motor STN. Through a combination of mechanisms, DBS of the STN seems to interrupt the disturbed informationprocessing, leading to a normalisation of connectivity within the cortico-basal gangliathalamocortical circuits and consequently to a reduction in symptoms. Based on the results of the studies described in this review, it can be concluded that the STN is a mechanism-based target for DBS in OCD.

Chapter 3 presents a case study of a patient with treatment-resistant OCD who underwent anteromedial STN-DBS. Despite a clear mood-enhancing effect, stimulation caused motor side-effects including bilateral hyperkinesia, dyskinesias and sudden large amplitude choreatic movements of arms and legs when stimulating at voltages above circa $1.5 \mathrm{~V}$. DBS at lower amplitudes and at other contact points failed to result in a significant reduction of obsessions and compulsions. To our knowledge, such severe and persistent motor side-effects have not yet been described for anteromedial STN-DBS. Due to the limitation in programming options, we decided to re-operate and target the ventral capsule/ventral striatum (VC/VS), which resulted in a substantial reduction in key obsessive and compulsive symptoms without serious sideeffects.

Chapter 4 aimed to investigate the role of $5-\mathrm{HT}$ on mood, emotional processing, inhibitory control and motor symptoms in PD patients with STN-DBS by temporarily reducing the level of available $5-\mathrm{HT}$ in the brain by acute tryptophan depletion (ATD) in a double-blind, placebo-controlled crossover study. Due to lagging inclusion the study was terminated after including 7 patients. In these 7 patients, we found that 5HT manipulation by ATD did not lead to dissociable effects on any of the outcome measures, suggesting that psychiatric side-effects following STN-DBS in PD patients cannot be directly linked to a reduction in 5-HT activity. These results, however, should be interpreted with caution given the small sample size. 
In Chapter $\mathbf{5}$ we aimed to explore whether characteristics of the surgical trajectory were associated with cognitive decline in PD patients one year following STN-DBS. A total of 49 PD patients who underwent bilateral STN-DBS were retrospectively analysed. Cognitive change scores were related to the number of microelectrode recording (MER) trajectories, the STN length as measured by MER, and cortical entry points. One year postoperatively, significant declines were found in several executive function tests: verbal fluency, Stroop Colour-Word test and Trail Making Test B (TMTB). Changes in TMT-B were associated with the coronal entry point in the right hemisphere. However, only 5 patients showed clinically relevant cognitive decline in TMT-B performance. These results imply that the use of MER does not impose an increased risk of cognitive decline after STN-DBS. Although the electrode passage through the right prefrontal lobe may contribute to subtle changes in executive function, only few patients showed clinically relevant cognitive decline, which implies that from a cognitive point of view, STN-DBS in PD may be considered a relatively safe procedure.

\section{Part 2}

In Chapter 6 the experience sampling method (ESM) was used to explore longitudinal associations between motor symptoms and mood states in a PD patient suffering from motor fluctuations. For this purpose, one patient collected ESM data for 34 consecutive days. Network analysis revealed that anxiety and cheerfulness had a central position within the symptom network. Higher anxiety was prospectively associated with increased rigidity and tremor, and with feeling down. Cheerfulness was associated with less tremor. Balance problems were not influenced by cheerfulness nor anxiety, but increased balance problems were associated with reduced cheerfulness at the next assessment. Feeling down did not influence self-reported motor symptom severity at the next assessment. Revealing longitudinal associations between motor and mood states based on ESM data may have relevance for treatment strategies, e.g. by establishing a personalised treatment plan.

Chapter 7 explored the usability of routinely collected ESM data over a brief period of time to detect associations between motor fluctuations, affective state, and contextual factors in PD patients with motor fluctuations on a group and individual level. Group analyses may reveal population based patterns that may lead to more general 
treatment or research recommendations, while individual analyses can be used to establish a personalised treatment plan. On a group level, multilevel analyses showed significant associations between all motor symptoms and positive affect. Being at home was associated with increased balance problems and rigidity. These associations were independent on whether a patient was in ON or OFF state. Analyses on an individual level revealed much less significant associations that mostly, but not always, were in line with the results on a group level. This exploratory study showed that ESM is able to reveal associations between affective state, motor symptoms and contextual factors in a group of PD patients with motor fluctuations, but less so in individual patients. Since the ultimate aim is to use ESM as an aid to personalise treatments, the sensitivity of the approach needs to be increased.

Chapter 8 describes the rationale and methodology of a prospective, two-centre randomised controlled trial (RCT) that aims to study the clinical effectiveness, alterations in brain circuitry, and cost-effectiveness of cognitive behavioural therapy (CBT) for anxiety in PD. PD patients with anxiety will be randomised to CBT treatment and clinical monitoring (intervention group) or to clinical monitoring only (control group). The CBT programme used in this study was specifically developed to address symptoms of anxiety in PD patients. Participants will undergo standardised clinical, cognitive and behavioural assessment at baseline and at 2 follow-up measurements, as well as resting-state functional magnetic resonance imaging and diffusion tensor imaging before and after the intervention. The primary outcome measure is changes in severity of anxiety symptoms. Secondary outcome measures involve long-term changes in anxiety symptoms, changes in functional and structural connectivity between limbic and frontal cortices, and cost-effectiveness of the treatment. If proven effective, this study will hopefully contribute to a better and evidence-based approach for these non-motor symptoms.

In chapter 9 we aimed to explore the needs of PD patients who suffer from anxiety and their caregivers regarding the treatment of anxiety. Three focus group interviews in two countries involving 12 PD patients and 8 caregivers were conducted. Besides discussing the content of the treatment, emphasis was put on the structure of the therapy and potential barriers of undergoing therapy. Based on the interviews and a review of the literature, a specialised CBT programme was developed. The programme 
focuses on general and Parkinson-specific anxiety symptoms and involves classical elements of CBT, such as psycho-education, relaxation, cognitive restructuring, problem solving strategies and exposure, but also personal habits that may influence levels of anxiety and a self-management plan to maintain gains and prevent relapse.

Chapter 10 summarises and discusses the main findings of all previous chapters in the light of current scientific research. Methodological considerations and limitations are addressed and clinical and scientific implications are provided. 
Neuropsychiatrische symptomen omvatten een breed scala aan symptomen, zoals een depressieve stemming, euforie, agitatie en agressie, angst, slaapstoornissen en disinhibitie. Deze symptomen komen veelvuldig voor in neurologische, neurodegeneratieve en psychiatrische stoornissen. Neuropsychiatrische symptomen zijn geassocieerd met een dysfunctie van cortico-basale ganglia-thalamocorticale circuits. De basale ganglia zijn een groep subcorticale kernen die met elkaar verbonden zijn, waaronder de nucleus caudatus, het putamen, de globus pallidus, de substantia nigra of zwarte kern en de nucleus subthalamicus (STN). In dit proefschrift richten we ons op twee stoornissen die geassocieerd zijn met veranderingen van cortico-basale ganglia-thalamocorticale circuits: de ziekte van Parkinson (ZvP) en obsessiefcompulsieve stoornis (OCS).

De ZvP is een veelvoorkomende neurodegeneratieve aandoening met een prevalentie van ongeveer 1-2\% bij mensen van 65 jaar en ouder. Naast de klassieke motorische symptomen, zoals rusttremor, bradykinesie, rigiditeit en posturale instabiliteit, hebben veel parkinsonpatiënten last van neuropsychiatrische symptomen zoals depressie en angst. $\mathrm{Er}$ is momenteel veel onduidelijk over de onderliggende mechanismen van stemmingssymptomen bij Parkinson. Tevens is er geen evidence-based behandeling beschikbaar voor angst bij Parkinson. OCS is een neuropsychiatrische stoornis die gekenmerkt wordt door terugkerende dwang-gedachten (obsessies) en dwanghandelingen (compulsies). Obsessies zijn ongewenste, zich opdringende en steeds terugkerende gedachten, beelden of impulsen. Compulsies zijn herhaalde handelingen of mentale acties die ten doel hebben om de obsessie onder controle te houden, ongemak en/of angst te verminderen of mogelijke negatieve gebeurtenissen te voorkomen. Diepe hersenstimulatie van de STN (STN-DBS) is een neurochirurgische ingreep voor zowel de ZvP als OCS. Het eerste deel van dit proefschrift richtte zich op het vergroten van kennis omtrent de rol van de STN bij de pathofysiologie van OCS en om de onderliggende mechanismen van emotionele en cognitieve bijwerkingen van STN-DBS bij parkinsonpatiënten te onderzoeken. Het tweede deel van dit proefschrift richtte zich op stemmingssymptomen bij de ZvP en had ten doel om de relatie tussen stemmings- en motorische symptomen bij de ZvP te onderzoeken, en om een psychotherapeutische behandeling voor angst bij Parkinson te ontwikkelen. 
Hoofdstuk 1 bevat een algemene inleiding in het onderwerp en de doelstellingen van dit proefschrift. Het bevat informatie over de achtergrond van de ZvP en OCS, (neuropsychiatrische) symptomen, behandelingen inclusief diepe hersenstimulatie, evenals de onderzoeksvragen en opbouw van dit proefschrift.

\section{Deel 1}

Hoofdstuk 2 bevat een review over de functionele rol van de nucleus subthalamicus (STN) in OCS. Op basis van literatuuronderzoek denken we dat de STN, via zijn centrale positie binnen de cortico-basale ganglia-thalamocorticale circuits, een coördinerende rol heeft bij besluitvormingsprocessen en bij de selectie van handelingen. Een verstoorde informatieverwerking op het niveau van de STN is verantwoordelijk voor de kernsymptomen van OCS. Onderzoek heeft aangetoond dat er sprake is van een elektrofysiologische dysfunctie in de associatieve en limbische (niet-motorische) gedeelten van de STN. Via een combinatie van mechanismen onderbreekt DBS de verstoorde informatieverwerking, wat leidt tot een normalisatie van de verbindingen binnen de cortico-basale ganglia-thalamocorticale circuits en uiteindelijk tot een vermindering van symptomen. Op basis van de resultaten die beschreven zijn in dit review kunnen we concluderen dan de STN een op mechanismen gebaseerde target voor DBS in OCS is.

Hoofdstuk 3 beschrijft een casus van een patiënt met therapieresistente OCS die DBS onderging in het anteromediale gedeelte van de STN. Ondanks een duidelijke stemmingsverbetering leidde stimulatie boven $1.5 \mathrm{~V}$ tot motorische bijwerkingen, inclusief bilaterale hyperkinesie, dyskinesie en plotselinge choreatische bewegingen van de armen en benen. DBS op lagere amplitudes of andere contactpunten leidde niet tot een vermindering in obsessies of compulsies. Voor zover we weten zijn zulke ernstige en persisterende motorische bijwerkingen na DBS van de anteromediale STN niet eerder beschreven. Vanwege de beperkingen in de DBS-programmering is besloten de patiënt opnieuw te opereren en de DBS te implanteren in de regio van de ventrale capsula en het ventrale striatum (VC/VS), wat resulteerde in een substantiële afname in obsessieve en compulsieve symptomen zonder significante bijwerkingen.

In Hoofstuk 4 hebben we gekeken naar de invloed van serotonine (5-HT) op stemming, emotionele informatieverwerking, inhibitie controle en motorische 
symptomen bij parkinsonpatiënten die worden behandeld met STN-DBS. Hiervoor hebben we in een dubbel-geblindeerde gerandomiseerde cross-over studie het serotoninegehalte tijdelijk kunstmatig verlaagd en stemmings- en gedragstaken afgenomen. We zijn vroegtijdig, na het includeren van 7 patiënten, met deze studie moeten stoppen, omdat weinig patiënten in aanmerking kwamen voor de studie of bereid waren deel te nemen. Bij deze 7 patiënten vonden we geen effecten van 5-HT manipulatie op stemming en gedrag, wat suggereert dat psychische bijwerkingen na STN-DBS in PD niet een direct gevolg zijn van een afname in 5-HT activiteit. De resultaten dienen echter met de nodige voorzichtigheid te worden geïnterpreteerd vanwege de lage steekproefomvang.

In Hoofdstuk 5 hebben we onderzocht of afnames in cognitief functioneren (gemeten als veranderscores op cognitieve taken) 1 jaar na STN-DBS in parkinsonpatiënten geassocieerd zijn met kenmerken van het chirurgische traject, waaronder het aantal microelectroden (MER) dat gebruikt werd voor finetuning van de doellokalisatie tijdens DBS, de anatomische lengte van het STN traject zoals gemeten door MER, en de corticale ingangspunten (coronaal en sagittaal). In deze retrospectieve studie werden 49 patiënten met ZvP die bilaterale STN-DBS hebben ondergaan geanalyseerd. Eén jaar na de operatie werden significante afnames gezien in een aantal taken die executief functioneren meten: verbal fluency, Stroop Colour-Word test en Trail Making Test B (TMT-B). Veranderingen in prestaties op de TMT-B waren geassocieerd met het coronale ingangspunt in de rechter hemisfeer. Echter bleek er maar bij 5 patiënten sprake te zijn van een klinisch relevante cognitieve afname in prestaties op de TMT-B taak. Deze resultaten impliceren dat het gebruik van MER niet tot een verhoogd risico op een afname in cognitief functioneren leidt na STN-DBS. Hoewel de passage van de DBS-electrode door de rechter prefrontale kwab mogelijk bijdraagt aan subtiele veranderingen in executief functioneren, lieten maar een klein aantal patiënten een klinisch relevante afname in cognitief functioneren zien, wat impliceert dat vanuit een cognitief oogpunt STN-DBS bij ZvP een relatief veilige procedure is.

\section{Deel 2}

In Hoofdstuk 6 hebben we gebruik gemaakt van de experience sampling methode (ESM) om longitudinale associaties tussen motorische en stemmingssymptomen te bestuderen in een parkinsonpatiënt met motorische fluctuaties. Gedurende 34 
achtereenvolgende dagen heeft 1 parkinsonpatiënt ESM data verzameld. Een netwerkanalyse toonde aan dat angst en opgewektheid een centrale positie hadden binnen het symptoomnetwerk van deze patiënt. En hogere mate van angst was prospectief geassocieerd met verhoogde stijfheid en tremor, en met zich down voelen. Opgewektheid was geassocieerd met verminderde tremor. Balansproblemen werden niet beïnvloed door opgewektheid of angst, maar een toename in balansproblemen was geassocieerd met een verminderde opgewektheid tijdens het volgende meetmoment. Zich down voelen was niet geassocieerd met de zelf-gerapporteerde ernst van motorische symptomen tijdens het volgende meetmoment. Het blootleggen van longitudinale associaties tussen motorische en stemmingssymptomen op basis van ESM data is relevant voor het bepalen van behandelstrategieën, bijvoorbeeld voor het maken van een persoonlijk behandelplan.

In Hoofdstuk 7 hebben we de bruikbaarheid van routinematig verzamelde ESM data onderzocht om op groeps- en individueel niveau associaties tussen motorische symptomen, affectieve symptomen en contextuele factoren in kaart te brengen bij parkinsonpatiënten met motorische fluctuaties. Groepsanalyses kunnen mogelijke patronen op populatieniveau aan het licht brengen die kunnen leiden tot meer algemene behandel- of onderzoeksaanbevelingen. Individuele analyses kunnen gebruikt worden om een gepersonaliseerd behandelplan tot stand te brengen. De analyse op groepsniveau liet significante associaties zien tussen alle motorische symptomen en positief affect. 'Thuis zijn' was geassocieerd met een toename in balansproblemen en rigiditeit. De associaties waren onafhankelijk van of een patiënt in een ON of OFF-fase was. De analyses op individueel niveau lieten veel minder significante associaties zien, die in de meeste gevallen, maar niet altijd, overeenkwamen met de resultaten op groepsniveau. Deze exploratieve studie toont aan dat ESM in staat is om op groepsniveau associaties tussen affectieve symptomen, motorische symptomen en contextuele factoren te weergeven, maar dat dit een stuk minder is bij individuele patiënten. Aangezien het uiteindelijke doel van ESM erop gericht is om ingezet te worden als hulpmiddel bij het personaliseren van behandelingen, is het van belang dat de sensitiviteit van de methode verhoogd wordt.

Hoofdstuk 8 beschrijft de rationale en methode van een prospectief, multi-centrum gerandomiseerd gecontroleerd onderzoek dat erop gericht is om de klinische 
effectiviteit, de kosteneffectiviteit en veranderingen in hersencircuits te onderzoeken van cognitieve gedragstherapie (CGT) voor angst bij de ZvP. Parkinsonpatiënten met angst worden verdeeld (gerandomiseerd) in twee groepen: CGT en klinische monitoring (interventie groep) of alleen klinische monitoring (controle groep). Het CGTprogramma dat in deze studie gebruikt wordt is specifiek ontwikkeld om angst bij parkinsonpatiënten te behandelen. Participanten zullen tijdens de start van de studie en tijdens twee follow-up momenten een gestandaardiseerde klinische, cognitieve en gedragsmeting ondergaan. Tevens vindt er zowel vooraf als na de studie MRIonderzoek plaats. De primaire uitkomstmaat zijn veranderingen in de ernst van angstsymptomen. Secundaire uitkomstmaten zijn veranderingen in angstsymptomen op de lange termijn, veranderingen in limbische en frontale hersencircuits, en kosteneffectiviteit. Indien aangetoond wordt dat de behandeling effectief is, zal deze hopelijk bijdragen aan een betere evidence-based behandeling voor angstsymptomen bij Parkinson.

In Hoofstuk 9 hebben we de behoeften van parkinsonpatiënten met angstklachten met betrekking tot de behandeling van angstklachten geëxploreerd. Hiervoor hebben we drie focusgroepen in twee landen georganiseerd waaraan 12 parkinsonpatiënten en 8 mantelzorgers hebben deelgenomen. Naast het bespreken van de inhoud van de behandeling zijn ook de structuur van de behandeling en mogelijke barrières om behandeling te ondergaan aan bod gekomen. Op basis van de interviews en een beoordeling van de literatuur hebben we een gespecialiseerd CGT-programma ontwikkeld. Het CGT-programma richt zich op algemene en Parkinson-specifieke angstsymptomen. Het bevat klassieke CGT-elementen, zoals psycho-educatie, ontspanningstechnieken, cognitief herstructureren, probleemoplossende strategieën en exposure, maar ook persoonlijke gewoonten die angstlevels kunnen beïnvloeden en een zelfmanagement plan om terugval te voorkomen.

In Hoofdstuk 10, de Algemene Discussie, zijn alle hoofdbevindingen uit de eerdere hoofdstukken samengevat. Daarnaast worden de methodologische aspecten, implicaties voor de klinische praktijk en aanbevelingen voor toekomstig onderzoek besproken. 


Knowledge valorisation 
This first part of this thesis focused on Deep brain stimulation (DBS) studies in obsessive-compulsive disorder (OCD) and Parkinson's disease (PD) in order to study underlying mechanisms of neuropsychiatric symptoms. The second part of this thesis focused on mood symptoms in PD by exploring the usability of the experience sampling method (ESM) in PD patients, and by developing a treatment programme for anxiety in PD patients. This valorisation paragraph describes how the obtained knowledge from the research in this thesis can be made valuable for clinical and societal use.

\section{Societal relevance}

Neuropsychiatric symptoms include a broad range of psychiatric symptoms that are believed to be mediated by changes in brain functions. Certain conditions that were once considered to be manifestations of psychodynamic conflict are now considered neuropsychiatric disorders, such as OCD. Moreover, it is increasingly recognised that neuropsychiatric symptoms, such as anxiety and a depressive mood, are an integral part of PD, the second most common neurodegenerative disorder.

Neuropsychiatric symptoms have a large impact on health-related quality of life, which reflects the impact of a disease or symptoms on several aspects of a patient's life, including their physical, mental and social well-being. Moreover, according to the World Health Organization (WHO), neuropsychiatric disorders are the third leading cause of disability-adjusted life years (DALYs). According to the National Institute of Mental Health, DALY is a measure of overall disease burden, expressed as the total number of years lost to illness, disability, or premature death within a given population. This high disease burden of neuropsychiatric symptoms has a considerable impact on the global economic burden. In PD, for example, neuropsychiatric symptoms complicate treatment and require additional and complex interventions.

Despite the impact of neuropsychiatric symptoms on the health status of patients and global health care costs, treatments specifically developed and validated for neuropsychiatric symptoms are still scarce. This is partly because underlying mechanisms of neuropsychiatric symptoms are largely unknown. The findings reported in this thesis improve our understanding of underlying mechanisms of neuropsychiatric symptoms in PD and OCD, which is crucial in order to adapt and tailor treatment. Moreover, new methods and interventions have been introduced to improve care for 
patients. Improved care will in turn contribute to an increase in quality of life of patients, and eventually to a decrease in health care costs and societal burden.

\section{Target audience}

Our findings are relevant for OCD and PD patients, clinicians, psychologists, health insurance companies and researchers.

The findings from the DBS studies (chapter 2 to 5 ) are relevant for patients, since the findings might improve the surgical procedure, and subsequently improve treatment outcome. Knowledge of the neural substrates underlying obsessive and compulsive symptoms (chapter 2), as well as observed side-effects following DBS of a specific target, the STN, (chapter 3) might help clinicians to achieve maximal therapeutic effect. The same holds for DBS for PD. Studying underlying mechanisms (chapter 4) and potential risk factors (chapter 5 ) of emotional and cognitive side-effects following DBS might be helpful for patient selection and improving the surgical procedure. In chapter 5 we show that neurosurgeons do not have to feel restrained by fear of cognitive sideeffects when using multiple MERs in order to attempt to have more possibilities for postoperative stimulation settings.

The findings from the ESM studies (chapter 6 and 7) are relevant for PD patients and their health care team, as ESM has to potential to improve care. Due to the unpredictable fluctuating nature of both motor and mood symptoms in PD, it is challenging to capture and study their association, and adjust treatment accordingly. In chapter 7 we showed that ESM allows to study associations between affective state, motor symptoms and contextual factors on a group level in PD. Findings on a group level may lead to more general treatment recommendations. Moreover, we showed that on an individual level ESM can be used to visualise and monitor symptom change over the course of the day. In chapter 6 we showed that ESM data collected over a longer period of time can be used to generate symptom networks displaying prospective associations between mood and motor symptoms. The information collected by ESM can lead to an increased awareness and understanding in patients and clinicians regarding particular circumstances in which symptoms occur or are perceived as more disabling. This might benefit collaborative decision making between the patient and clinician, help personalising treatment and give the patient a 'sense of 
control'. Our findings could also be of interest to insurance companies and policy makers, given that ESM has proven potential as an E-health tool for PD patients.

Finally, results from the CBT studies are valuable for PD patients who suffer from anxiety, psychologists working with these patients, health insurance companies and researchers. The CBT treatment, if proven effective, will hopefully provide PD patients with behavioural and anxiety management techniques that may give lasting benefits on anxiety symptoms, well-being, quality of life, and possibly on motor symptoms. The findings from the focus group interviews are particularly relevant for psychologists working with PD patients, as it provides insight into the specific needs of PD patients and potential barriers of undergoing therapy. Psychologists might tailor their method based on these findings. Moreover, the paper provides a detailed description of the CBT programme that was developed for PD patients suffering from anxiety, including factors that may complicate treatment, as well as ways how to deal with these during therapy. On top of that, the workbook and manual for therapists are available upon request in three different languages and we aim to develop a website to distribute our manual, so we hope that PD patients around the world benefit from the therapy. While the paper discussing the effectiveness of the intervention is currently in preparation, the findings are relevant for health insurance companies, as most health insurance companies apply evidence-based approaches to coverage decisions. Finally, the protocol describing the methodology of the study (chapter 8) might be relevant for researchers by facilitating the development of similar trials studying the effect of psychotherapeutic interventions for different target groups.

\section{Products/innovation}

The research described in this thesis can be considered innovative in several ways. First of all, we were the first to report severe and persistent motor side-effects following STN-DBS in an OCD patient. Reporting these side-effects are of crucial importance, as it might guide neurosurgeons in target selection.

Another innovative approach within this thesis concerned the usability of ESM in PD patients. The ESM method originated in psychiatry, and has recently been applied in a number of other diseases, such as migraine, stroke and COPD. In this thesis, we demonstrated that ESM is useful to study associations between motor and mood 
symptoms, and contextual factors on a group and individual level in PD. Moreover, we were the first to study prospective associations between motor and mood symptoms in $P D$ in an $n=1$ design. The findings demonstrate how recall bias can be prevented and they bring us one step closer to personalise treatment to the individual patient.

Despite its high prevalence, there is currently no evidence-based treatment available for anxiety in PD. Psychotherapeutic interventions that are available have not been studied in a controlled design or only in small sample sizes. The primary focus of our study was to assess the clinical effectiveness of a specialised CBT programme for anxiety in PD and possible changes in cerebral connectivity (chapter 8). The development of the CBT programme was based on literature, existing CBT modules and focus group interviews with patients, in order to tailor the programme to better serve their specific needs, concerns and circumstances (chapter 9). This study is the first in its kind to test both CBT effectiveness in a controlled trial and to record possible structural and functional brain changes induced by CBT, which may lead to an increased understanding of the underlying neurobiological mechanisms of both anxiety and response to treatment in PD patients.

\section{Implementation}

The knowledge acquired from the studies in this thesis will be implemented in health care practice on one hand, and used for continuation of research on the other hand. The findings from the OCD studies contributed to a shift in target selection for DBS in our hospital from the STN to the ventral capsule/ventral striatum. Moreover, a database has been created for all OCD patients who received DBS in our hospital, in order to collect data regarding target location, active electrodes, symptom improvement, sideeffects, medication, etc. This dataset is now used for research purposes with the aim to uncover best practices and improve outcome. The findings from the studies regarding the cognitive side-effects following STN-DBS in PD resulted in the continuation of the use of microelectrode recording as a way of optimising lead implantation. Moreover, we continue to collect data from PD patients who received STN-DBS in order to increase our sample size and apply advanced statistical methods and machine learning algorithms to discover underlying mechanisms of side-effects and clinical outcome. 
The application of ESM in the field of PD care is still in its infancy. This thesis provides a valuable contribution to our knowledge of the usability of ESM for research purposes and health care practice in PD. ESM can be used to capture the fluctuating nature of motor and mood symptoms in PD and to study their association in relation to contextual factors. Moreover, revealing population based patterns via ESM may lead to more general treatment recommendations, while individual analyses of ESM data can be used to establish a personalised treatment plan. However, we also found that the sensitivity of the method needs to be improved for further implementation, for which we have made several recommendations. In the future, ESM data may potentially serve as a parallel ground truth to objective data, such as obtained by monitoring motor symptoms by wearable sensors, since these alone may not be representative for the experienced disability. In addition, combining objective and subjective data on motor and non-motor symptoms can potentially be used as input signal for adaptive DBS. Adaptive DBS aims to automatically adapt stimulation parameters to the fluctuating clinical state of the patient, where stimulation is only provided when necessary. The idea is that adaptive DBS results in fewer stimulation-induced side-effects compared to conventional DBS.

The CBT programme aimed at treating anxiety in PD is currently in use in our hospital. To disseminate our findings among health care professionals we gave presentations and workshops at conferences. Moreover, we presented our programme at several Parkinson café's in the region in order to create awareness regarding anxiety and its treatment among PD patients and health care professionals. The CBT programme, including the workbook and manual for therapists, is available in three different languages so we hope it will find its way into widespread clinical use. We are currently finalising our paper regarding the clinical effectiveness of the programme. Meanwhile, we are studying the cost-effectiveness of the treatment, as well as changes in cerebral connectivity associated with successful treatment in order to unravel the underlying neurobiological mechanisms of anxiety in PD. 

Dankwoord 
Het is eindelijk zover, mijn proefschrift is af! Het was een bijzondere tijd met veel leerzame, stressvolle, maar vooral leuke momenten. Ik wil iedereen die heeft bijgedragen aan dit proefschrift en me gesteund heeft van harte bedanken.

Allereerst wil ik alle mensen die in de afgelopen jaren deel hebben genomen aan mijn studies heel erg bedanken. Jullie openheid, betrokkenheid, interesse, maar vooral veerkracht, was een enorme bron van inspiratie! Daarnaast ook een diepe buiging voor alle mantelzorgers die indirect hebben bijgedragen aan de studies. Zonder de vele autoritjes en het lange wachten totdat jullie dierbare klaar was met deelname aan mijn studies was dit proefschrift niet tot stand gekomen, bedankt!

Daarnaast wil ik mijn promotieteam, bestaande uit prof. dr. Yasin Temel, prof. dr. Albert Leentjens en dr. Anja Moonen hartelijk bedanken:

Beste Yasin, bedankt dat je mij de kans hebt gegeven om als student onderzoek te doen binnen jouw team en om de stap te zetten naar promotieonderzoek. Als enige 'psycholoog' in het team die vooral klinisch-georiënteerd onderzoek deed, was ik in eerste instantie een vreemde eend in de bijt en was het even zoeken naar mijn plek. Ik waardeer het ontzettend dat je mij de ruimte hebt gegeven om keuzes te maken die bij mij passen en die goed waren voor mijn toekomst.

Beste Albert, bedankt voor je begeleiding en bijdrage aan dit promotieonderzoek. Ik weet nog dat we tijdens onze eerste ontmoeting beiden afzonderlijk een lijstje hadden gemaakt met onderwerpen die we moesten bespreken: het was identiek! Je kritische blik en je vlotte en uitgebreide commentaar op papers hebben mij ontzettend geholpen. Bedankt voor je geduld en vertrouwen in mij!

Anja, onze samenwerking begon toen jij met zwangerschapsverlof was. Ik moest een groot (en leuk) project overnemen. Het was in het begin een hele uitdaging, iets mee ontwikkelen zonder goed te weten wat jij voor ogen had en het proberen zo goed mogelijk te doen. Maar naar mijn gevoel is het als vanzelf gegaan. Niet lang nadat je terug was voelde de samenwerking voor mij als een geoliede machine. Later werd je mijn co-promotor, een andere rol die ook als vanzelf gegroeid is. Het waren gezellige gesprekken met een koffie erbij (dat moet ook), maar tegelijkertijd momenten waar ik even op adem kon komen en mijn ei kwijt kon. Je hebt altijd stil gestaan bij hoe het 
met me gaat en bewaakt dat ik niet te veel hooi op mijn vork nam. Dank voor je steun en wat tof dat we nu als collega's bij de Medische Psychologie een kantoor en vele koffiemomenten delen.

Leden van de beoordelingscommissie, hartelijk dank voor het lezen en beoordelen van mijn proefschrift.

Beste Annelien, een speciaal woord van dank voor jou. Ik heb je leren kennen toen ik nog bachelor student was en vanaf het begin heb je mij onder je hoede genomen, zowel bij het begeleiden van onderzoek als bij het klinische werk. Jou zien werken met patiënten is ontzettend inspirerend. Overal waar ik kom, wordt met lof over je gesproken, en terecht! Je bent mijn grote voorbeeld. Dankjewel voor alle leerzame, licht chaotische en gezellige gesprekken en dat je me de kans hebt gegeven om te groeien op zowel persoonlijk als professioneel vlak. Ik zal onze fantastische trip naar de Davos sessies in Zwitserland nooit vergeten, wat was dat gezellig! Op nog vele toekomstige samenwerkingen!

Dank aan de overige leden van het DBS-team die me geholpen hebben bij de inclusie van patiënten voor mijn onderzoeken, het verzamelen van data en samples of het schrijven van artikelen: Nicole Bakker, Mark Kuijf, Mark Janssen, Mayke Oosterloo, Linda Ackermans, Pieter Kubben, Koen Schruers, Mirella Waber en Daisy Ramakers. Naast jullie praktische bijdrage heb ik geregeld met jullie mee mogen kijken tijdens jullie werkzaamheden. Ik heb hier ontzettend veel van geleerd. Bedankt voor deze belangrijke bijdrage!

Ik wil graag alle co-auteurs bedanken die bij hebben gedragen aan de totstandkoming van de artikelen in dit proefschrift. Marjan Drukker, jouw kennis en ervaring op het gebied van ESM was van onschatbare waarde. Bedankt voor de vlotte en gedetailleerde antwoorden op al mijn vragen en je statistische bijdrage aan de ESMstudies. Jos Adam, heel erg bedankt voor je uitgebreide uitleg bij de anti-cue task en je begeleiding bij de analyses. Marjolein de Vugt, dank voor je klinische supervisie bij de CBT-studies, waar ik voor het eerst behandelervaring als psycholoog heb opgedaan. Je begeleiding was ontzettend fijn en leerzaam! 
Ron Mengelers en Nico Rozendaal, bedankt voor jullie belangrijke ondersteuning bij de verschillende studies. Niet alleen hebben jullie geholpen met het ontwikkelen van programma's, bij iedere stap dachten jullie met ons mee. Dat was ontzettend fijn!

Janine Portier, Colin van der Heijden en Rianne Esselink: samen met Annelien hebben we met veel passie aan het Beter in Balans project gewerkt. Dit belangrijke project gericht op het verbeteren van de psychosociale zorg rondom een DBS-operatie is geen onderdeel van dit proefschrift, maar heeft wel een zeer belangrijke bijdrage geleverd aan mijn visie op de zorg. Dank jullie wel voor de ontzettend prettige en gezellige samenwerking. Jullie zijn stuk voor stuk inspirerend en ik hoop in de toekomst nog veel vaker met jullie samen te werken.

Rachel, Rowan, Anne en Laura, heel erg bedankt voor jullie hulp als student bij de verschillende projecten in dit proefschrift.

Collega's van de afdeling Neurochirurgie van MHeNS wil ik bedanken voor hun ondersteuning tijdens mijn promotieonderzoek. Gedurende mijn tijd als PhD-student is het team gegroeid van, 'we passen nog allemaal in een kantoor tijdens de Monday morning meeting' tot 'er is geen zaal beschikbaar die ruim genoeg is voor iedereen'. De afgelopen jaren heb ik vanwege mijn overige werkzaamheden wat meer afstand genomen van de groep, maar ik wil toch even stilstaan bij een paar naaste collega's en wat voor mij de mooiste momenten van mijn PhD waren: dear Sarah, dear friend. We started as roomies, somewhere along the road introduced babyshowers to the group (I will never forget Yasin's face when he walked into the MPR room with 3 other neurosurgeons he wanted to show the lab, hilarious!), and became the 'mama's of the group'. Thank you for always being there, for the good and the bad moments. Without your support, I could not have done it. Now let's finally plan that playdate with the kids!

Milaine, Millie, Mil, wat ben $\mathrm{jij}$ toch een tof mens. Je humor en gevatte opmerkingen kwamen altijd op de momenten dat ze het hardst nodig waren. Met veel bewondering kijk ik terug naar jouw doorzettingsvermogen, je veelzijdige skills (je bent ook goed met een hamer), maar vooral hoe je onverstoord door kon werken op momenten dat Fred, Gusta en ik onze mond niet konden houden. Dank je wel voor alles! 
Freddie, Fred (Fred Fred Fred Fred), monsieur! Nooit vergeet ik onze introductiedag op het lab (hier hoef ik me niet verder over uit te wijden). We zijn op dezelfde dag aan onze PhD begonnen en hebben de eerste jaren een kantoor gedeeld. Hierdoor kregen we de goede en slechte momenten van elkaar mee. Dank voor je steun en wat ik vooral onthoud is dat er niets is dat niet opgelost kan worden met een heliumballon, een fles whisky, een lekker etentje of een marsepeinen varken.

Gusta, Gustie, mijn lieve roomie, vriendin en tevens paranimf. Jij bent echt een van de meest sprankelende personen die ik ken. Ja, zelf als je nors en wild achter je laptop zat te typen, bracht je een glimlach op ons gezicht. Van veel werken kwam het niet altijd als je weer terug kwam bij ons op kantoor na een huisartsenstage (totdat je er genoeg van had en een BOSE noisecancelling headphone aanschafte), maar echt, het was onvergetelijk: het spiegelen met Fred, de congrestripjes, dat sauna bezoek in Duitsland (omg), het samen koken, de vele wijntjes, yoga op kantoor, een promotiefilm met barbies en stiekeme filters, golfen met alpaca's, maar vooral ook hele fijne gesprekken. Dank je wel voor je steun en alle gezellige momenten.

Dr. Majed, what a personality you are, so generous and always smiling! You made every birthday in the group a true feast with glitters and big cakes. The dinners at your place were over the top fantastic, and who knew you could run that fast! I will never forget that moment on the bridge at our conference trip to Cologne. I am truly happy to know you. Jeroen, dank je dat je weer wat gezelligheid terugbracht in het team nadat er zo velen waren weggegaan. Jij introduceerde weer de bbq's, etentjes, lekkere drankjes (vaak eigengemaakt) die we allen hard nodig hadden. Beth, wow, I haven't seen you for ages. I hope you are happy and shining in Amsterdam. Liancy Peng, we were all sad that you left us, but happy that you found a PhD position that better fitted your background and preferences. It was so much fun having you as a colleague. Margot, Sylvana, Jackson, Jana, Roman, Christian, Faris, Mohammed, Amee, Stijn, Faisal, Raghu and old colleagues, thank you all for the good times and support! A special word to the two ladies from Barcelona. Dear Sol, you are such a fantastic person. We shared some very special moments during your stay here that I will never forget! Thank you for your kindness, support and friendship. Maria, your presence just lighted up the room during your stay with us. You are such a smart, kind and lovely person. You are going to be a great doctor. 
Govert, dank je wel voor je oprechte interesse in ieders project en je kritische wijze van vragen stellen. Het hield de kwaliteit van onze wetenschappelijke meetings hoog en stimuleerde ons om constant up-to-date te blijven. Mark, ondanks dat je een steeds prominentere rol kreeg binnen onze groep, heb je je nooit te goed gevoeld om uren achter een computer te zitten en een database te vullen (uiteraard met een goede koffie erbij). Bedankt voor je steun, interesse en hulp bij de verschillende projecten. Ali, dear friend, from the beginning of my PhD you were so kind to me. Thank you for all your support these years!

Lieve Danique en Elles, mijn roomies in de tweede periode van mijn promotieonderzoek. We kennen elkaar al sinds onze stage bij de geheugenpoli en zijn gewoon verder gegaan waar we gebleven waren. Wat hebben we het toch gezellig met elkaar gehad: de uitspraken op de whiteboards, de krokettenteller, de mooiste Kerstversiering, de altijd aanwezige chocolade op kantoor, de fijne gesprekken. Wat fijn dat we alles met elkaar konden delen. Elles, jij verliet ons als eerste voor een nieuwe baan bij MetGGZ, maar het lot bracht ons al gauw voor de derde keer weer bij elkaar. Wat geweldig dat we nu samen de opleiding tot GZ-psycholoog volgen en weer klasgenoten zijn. Lieve Danique, mijn paranimf! Wat hebben we toch veel meegemaakt gedurende de afgelopen periode. Ik heb zo veel bewondering voor jouw doorzettingsvermogen en daadkracht. Je bent inmiddels ook klaar met je PhD en hebt al een mooie baan als psycholoog. Ergens voel ik dat we ooit weer collega's worden, maar voor nu waardeer ik onze vriendschap heel erg.

Niky, Liselot, Sanne, dank jullie wel voor de gezelligheid en de heerlijke etentjes. Liselot, wat hebben we gelachen tijdens de summer course in Toscane, die cheesecake, Ik kan nog huilen van het lachen als ik de foto's terugzie.

Mijn liefste vriendinnen: Anna, Eva, Madelon, Merel, Renee. De verhalen over mijn $\mathrm{PhD}$ waren vast niet altijd even interessant, maar bedankt voor het aanhoren en voor de zo nodige ontspanmomenten en gezelligheid tussen door.

Maarten en Lieke, bedankt voor jullie steun en hulp de afgelopen jaren. Dat jullie altijd in mij geloofd hebben, betekent veel voor me. 
Lieve pap en mam, misschien begrepen jullie niet altijd tot in detail waar mijn studies over gingen, maar jullie zijn altijd heel geïnteresseerd geweest in hoe het allemaal ging en ik heb altijd gevoeld hoe trots jullie op me zijn. Ik kan altijd bij jullie terecht en jullie steun, ook de praktische, is van onschatbare waarde voor mij. Zonder jullie was me dit niet gelukt, dank jullie wel!

Dear Ehsan, you are by far the best part of this PhD story. Who would have guessed I would find my husband at the corridor of division 3. You have been my rock throughout this period. You have always believed in me and are my biggest supporter. I can not put into words how much your support and love means to me. Thank you for everything! Sophia, my little sunshine, mijn krullebolletje, your smile always lights up the room and made me forget about all my PhD frustrations in a second. Wat ben ik toch gek op jou. 

Curriculum Vitae 
CURRICULUM VITAE 


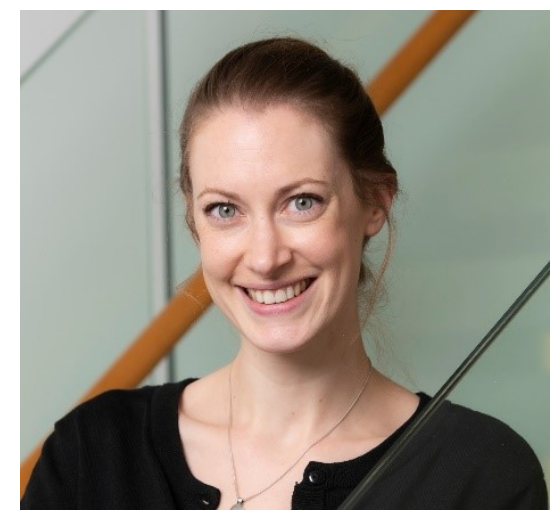

Anne Evangelia Philomena Mulders was born on December 22, 1986 in Heerlen. After graduating from high school in 2005, she started to study Pedagogical studies at Fontys University of Applied Sciences. Following her study, she worked as a support worker in an organisation that provides care and support for people with intellectual disabilities. In 2010, she started to study Health Sciences with a focus on Mental Health at Maastricht University. After obtaining her bachelor degree in 2013 (cum laude), she obtained a master degree in Neuropsychology in 2014 (cum laude) and a master degree in Mental Health in 2015 (cum laude), at Maastricht University. During her master degrees she performed a clinical internship at the memory clinic of Maastricht University Medical Center (MUMC+), as well as a research internship studying deep brain stimulation (DBS) for Parkinson's Disease (PD). In 2015, she started working as a PhD candidate at the department of Psychiatry and Neuropsychology and the department of Neurosurgery under supervision of prof. dr. Albert Leentjens, prof. dr. Yasin Temel and dr. Anja Moonen. During her PhD she focused on cognitive and emotional side-effects following DBS in PD, the relationship between motor and mood symptoms in PD, and the treatment of anxiety in PD. On top of that, she studied DBS for obsessieve-compulsive disorder. Next to her PhD trajectory, she was involved in the development and implementation of several psychosocial interventions for PD patients and their caregivers: 'Parkinson Partner in Balans', an online self management programme for caregivers of PD patients, and 'Beter in Balans', a psychosocial intervention aimed at supporting PD patients and their partners before, during and after DBS surgery. Currently she is working as a Healthcare psychologist trainee at the Medical Psychology department of MUMC+. 

Publications 
PUBLICATIONS 
Mulders AEP, Plantinga BR, Schruers $\mathrm{K}$, et al. Deep brain stimulation of the subthalamic nucleus in obsessive-compulsive disorder: Neuroanatomical and pathophysiological considerations. Eur Neuropsychopharmacol 2016;26(12):19091919.

Mulders AEP, Leentjens AFG, Schruers K, Duits A, Ackermans L, Temel Y. Choreatic Side Effects of Deep Brain Stimulation of the Anteromedial Subthalamic Nucleus for Treatment-Resistant Obsessive-Compulsive disorder. World Neurosurg 2017;104:1048 e1049-1048 e1013.

Mulders AEP*, Moonen AJH*, Dujardin $\mathrm{K}$, et al. Cognitive behavioural therapy for anxiety disorders in Parkinson's disease: Design of a randomised controlled trial to assess clinical effectiveness and changes in cerebral connectivity. J Psychosom Res 2018;112:32-39. *shared first author

Mulders AEP*, van der Velden RMJ*, Drukker M, Kuijf ML, Leentjens AFG. Network analysis of symptoms in a Parkinson patient using experience sampling data: $A n n=1$ study. Mov Disord 2018;33(12):1938-1944. *shared first author.

Mulders AEP*, van der Velden RMJ*, Drukker M, Broen MPG, Kuijf ML, Leentjens AFG. Usability of the Experience Sampling Method in Parkinson's Disease on a Group and Individual Level. Mov Disord 2020; 35(7): 1145-1152. *shared first author.

Mulders AEP, Temel Y, Tönge M, Schaper F, van Kranen-Mastenbroek V, Ackermans $L$, Knubben $P$, Janssen MLF, Duits AA. The association between surgical characteristics and cognitive decline following deep brain stimulation of the subthalamic nucleus in Parkinson's disease. (Submitted)

Mulders AEP, Leentjens AFG, Dujardin K, Flinois B, Leterme A-C, Duits AA, de Vugt $M$, Kuijf ML, Moonen AJH. Cognitive behavioural therapy for anxiety disorders in Parkinson's disease: development and content of a specialised and tailored treatment programme. (Submitted)

Roet M, Boonstra J, Sahin E, Mulders AEP, Leentjens AFG, Jahanshahi A. Deep Brain Stimulation for treatment-resistant depression: towards a more personalized treatment apprach. J. Clin. Med. 2020, 9(9), 2729.

Habets JGV, Duits AA, Sijben LCJ, Mulders AEP, de Greef B, Temel Y, Kuijf ML, Kubben PL, Herff C, Janssen MLF. Machine learning prediction of motor response after deep brain stimulation in Parkinson's disease - proof of principle in a retrospective cohort. Submitted. 

Thesis defenses from MHeNs 


\section{3}

Rob Havermans: Bipolar disorder in daily life; Mood and cortisol responses to naturally occurring events. Supervisor: Prof.dr. M. de Vries; Co-Supervisor: Dr. N. Nicolson.

Véronique Moers-Hornikx: Deep brain stimulation and the cerebellum. Supervisors: Prof.dr. J. Vles / Prof.dr. Y. Temel; Co-Supervisor: Dr. G. Hoogland.

Nicole Veldhorst-Janssen: Intranasal delivery of rapid acting drugs. Supervisors: Prof.dr. M. Marcus / Prof.dr. C. Neef; Co-Supervisor: Dr. P.H. van der Kuy.

Stéphanie Knippenberg: Vitamin D and Multiple Sclerosis: immunological and clinical outcome. Supervisor: Prof.dr. J. Cohen-Tervaert; Co-Supervisors: Dr. J. Damoiseaux / Dr. Y. Bols.

Erik D. Gommer: Dynamic Cerebral Autoregulation: from methodology towards clinical application. Supervisors: Prof.dr. W.H. Mess / Prof.dr. R.B. Panerai, UK; Co-Supervisor: Dr.ir. J.P.H. Reulen.

Olga A.H. Reneerkens: Can PDE inhibition improve cognition? Translational insights. Supervisor: Prof.dr. H.W.M. Steinbusch; Co-Supervisor: Dr. J. Prickaerts.

Lyzel S. Elias-Sonnenschein: Clinical and biomarker correlates of genetic risk factors for Alzheimer's disease. Supervisor: Prof.dr. F.R.J. Verhey; Co-Supervisor: Dr. P.J. Visser.

Diego F. Mastroeni: Epigenetic Dysregulation and the Pathophysiology of Alzheimer's Disease. Supervisors: Prof.dr. H.W.M. Steinbusch / Prof.dr. P.D. Coleman, Sun City, Arizona; Co-Supervisors: Dr. B.P.F. Rutten / Dr. D.L.A. van den Hove.

Leonidas Chouliaras: Epigenetic Regulation in Aging and Alzheimer's disease: A translational perspective. Supervisor: Prof.dr. H.W.M. Steinbusch; Co-Supervisors: Dr. B.P.F. Rutten / Dr. D.L.A. van den Hove.

Liesbeth Knaepen: Perinatal events and altered pain sensitivity in later life. Supervisors: Prof.dr. E.A.J. Joosten / Prof.dr. D. Tibboel, EUR; Co-Supervisor: Dr. J. Patijn.

Marisela Martinez-Claros: Hippocampal plasticity and corticosterone: From dendrites to behaviour. Supervisor: Prof.dr. H.W.M. Steinbusch; Co-Supervisors: Dr. J.L. Pawluski / Dr. J. Prickaerts.

Marcus D. Lancé: A circle of improvement in bleeding management: from laboratory to clinic and back. Supervisors: Prof.dr. M.A.E. Marcu / Prof.dr. J.W.M. Heemskerk; Co-Supervisor: Dr. Y.M.C. Henskens.

Hilde Braakman: Imaging the brain; neuronal correlates of cognitive impairment in children with frontal lobe epilepsy. Supervisors: Prof.dr. A.P. Aldenkamp / Prof.dr. J.S.H. Vles; Co-Supervisors: Dr.ir. W.H. Backes / Dr. P.A.M. Hofman.

Willem H. van Zwam: Aneurysmal subarachnoid hemorrhage: imaging strategies and costeffectiveness aspects in diagnostic work-up and post-therapeutic follow-up. Supervisors: Prof.dr. J.T. Wilmink / Prof.dr. J.E. Wildberger; Co-Supervisor: Dr. P.A.M. Hofman.

Klara De Cort: The Pathogenesis of Panic Disorder. Supervisors: Prof.dr. I. Myin-Germeys / Prof.dr. E.J.L. Griez; Co-Supervisors: Dr. K.R.J. Schruers / Dr. I. Van Diest, Leuven. 
Kim van Wijck: Mind the Gap; experimental studies on splanchnic hyperfusion and gastrointestinal integrity loss in man. Supervisors: Prof.dr. W.A. Buurman / Prof.dr. C.H.C. Dejong; Co-Supervisor: Dr. K. Lenaerts.

Yvette Roke: Antipsychotic-induced hyperprolactinemia in children and adolescents with mainly autism spectrum disorders. Prevalence, symptoms, clinical consequences and genetic risk factors. Supervisors: Prof.dr. P.N. van Harten / Prof.dr. J.K. Buitelaar (RUN); Co-Supervisor: Dr. A. Boot (UMCG).

Fleur Goezinne: Retinal detachment surgery: pre and postoperative prognostic factors. Supervisors: Prof.dr. F. Hendrikse / Prof.dr. C.A.B. Webers; Co-Supervisor: Dr. E.C. La Heij (Amsterdam).

Ralph L.J.G. Maassen: The Merits of Videolaryngoscopy during Glottic Visualisation for Endotracheal Intubation. Supervisors: Prof.dr. M. Marcus / Prof.dr. A. van Zundert (University of Queensland).

Maria J. de Sousa Guerreiro: The role of sensory modality in age-related distraction. Supervisor: Prof.dr. C.M. van Heugten; Co-Supervisor: Dr. P.W.M. van Gerven.

Ine Rayen: Effects of developmental fluoxetine exposure on neurobehavioral outcomes. Supervisor: Prof.dr. H.W.M. Steinbusch; Co-Supervisors: Dr. J.L. Pawluski / Dr. T.D. Charlier (Ohio University, USA).

Nynke M.G. Bodde: Psychogenic non-epileptic seizures; a separate disorder or part of a continuum? Supervisors: Prof.dr. R. van Oostenbrugge / Prof.dr. K. Vonck (UZ Gent); Co-Supervisors: Dr. R. Lazeron / Dr. A. de Louw (Epilepsiecentrum Kempenhaeghe, Heeze).

Alejandro M. Gomez: Novel strategies for making myasthenia less gravis: targeting plasma cells and the neuromuscular junction. Supervisor: Prof.dr. M.H. De Baets; Co-Supervisors: Dr. M. Losen / Dr. P. Martinez-Martinez.

Mohammad S. Rahnama'i: Prostaglandins and Phosphodiesterases in the Urinary Bladder Wall. Supervisors: Prof.dr. Ph. Van Kerrebroeck / Prof.dr. S. de Wachter (Universiteit Antwerpen); CoSupervisor: Dr. G. van Koeveringe.

Mariken B. de Koning: Studying biomarkers in populations at genetic and clinical high risk for psychosis. Supervisors: Prof.dr. T. Amelsvoort / Prof.dr. J. Booij (AMC).

Fabien Boulle: Epigenetic regulation of BDNF/TrkB signaling in the pathophysiology and treatment of mood disorders. Supervisors: Prof.dr. H.W.M. Steinbusch / Prof.dr. L. Lanfumey (Universiteit Parijs); Co-Supervisors: Dr. D. van den Hove / Dr. G. Kenis.

\section{4}

Iris Nowak-Maes: Tinnitus; assessment of quality of life \& cost-effectiveness. Supervisors: Prof.dr. M. Peters / Prof.dr. B. Kremer; Co-Supervisors: Dr. M. Joore / Dr. L. Anteunis.

Marjolein Huijts: Cognitive function in patients with cerebral small vessel disease. Supervisor: Prof.dr. R.J. van Oostenbrugge; Co-Supervisors: Dr. A.A. Duits / Dr. J. Staals.

Markus Gantert: Fetal inflammatory injury as origin of long term disease: Lessons from animal models. Supervisors: Prof.dr. B. Kramer / Prof.dr. L. Zimmermann; Co-Supervisor: Dr. A. Gavilanes. 
Elke Kuypers: Fetal development after antenatal exposures: Chorioamnionitis and maternal glucocorticoids. Supervisors: Prof.dr. B.W. Kramer / Prof.dr. H.W. Steinbusch / Prof.dr. Suhas G. Kallapur (University of Cincinnati, Ohio, USA).

Pieter Kubben: Ultra low-field strength intraoperative MRI for Glioblastoma Surgery. Supervisor: Prof.dr. J.J. van Overbeeke; Co-Supervisor: Dr. H. van Santbrink.

Laura Baijens: Surface electrical stimulation of the neck for oropharyngeal dysphagia in Parkinson's disease: therapeutic aspects and reliability of measurement. Supervisor: Prof.dr. B. Kremer; Co-Supervisor: Dr. R. Speyer, Townsville.

Janneke Hoeijmakers: Small fiber neuropathy and sodium channels; a paradigm shift. Supervisor: Prof.dr. R.J. van Oostenbrugge; Co-Supervisors: Dr. C.G. Faber / Dr. I.S.J. Merkies.

Stephanie Vos: The Role of biomarkers in preclinical and prodromal Alzheimer's disease. Supervisor: Prof.dr. F.R. Verhey; Co-Supervisor: Dr. P.J. Visser.

Muriël Doors: The Value of Optical Coherence Tomography in Anterior Segment Surgery. Supervisors: Prof.dr. R.M. Nuijts / Prof.dr. C.A. Webers; Co-Supervisor: Dr. T.T.J.M. Berendschot.

Anneke Maas: Sleep problems in individuals with genetic disorders associated with intellectual disability. Supervisors: Prof.dr. I. Curfs / Prof.dr. R. Didden.

Sebastiaan van Gorp: Translational research on spinal cord injury and cell-based therapies; a focus on pain and sensorimotor disturbances. Supervisors: Prof.dr. B. Joosten / Prof.dr. M. van Kleef; Co-Supervisors: Dr. J. Patijn / Dr. R. Deumens, KU Leuven.

Andrea Sannia: High risk newborns and brain biochemical monitoring. Supervisor: Prof.dr. J.S.H. Vles; Co-Supervisors: Dr. D. Gazzolo, Alessandria, Italy / Dr. A.W.D. Gavilanes.

Julie A.D.A. Dela Cruz: Dopamine mechanisms in learning and memory: Evidence from rodent studies. Supervisors: Prof.dr. H.W.M. Steinbusch / Prof.dr. R.J. Bodnar, New York; Co-Supervisor: Dr. B.P.F. Rutten.

René Besseling: Brain wiring and neuronal dynamics; advances in MR imaging of focal epilepsy. Supervisors: Prof.dr. A.P. Aldenkamp / Prof.dr.ir. W.H. Backes; Co-Supervisor: dr. J.F.A. Jansen.

Maria Quint-Fens: Long-term care after stroke; development and evaluation of a longterm intervention in primary care. Supervisors: Prof.dr. J.F.M. Metsemakers / Prof.dr. C.M. van Heugten / Prof.dr. M. Limburg, Almere; Co-Supervisor: dr. G.H.M.I. Beusmans.

Veronique Moulaert: Life after survival of a cardiac arrest; the heart of the matter. Supervisors: Prof.dr. J.A. Verbunt / Prof.dr. C.M. van Heugten / Prof.dr. D.T. Wade, Oxford, UK.

Feikje Smeets: The hallucinatory-delusional state: a crucial connection in the psychosis symptom network. Supervisor: Prof.dr. J. van Os; Co-Supervisor: Dr. T. Lataster.

Lies Clerx: Alzheimer's disease through the MR-eye; novel diagnostic markers and the road to clinical implementation". Supervisor: Prof.dr. F. Verhey; Co-Supervisors: Dr. P.J. Visser / P. Aalten.

Sonny Tan: The subthalamic nucleus in Parkinson's disease. Supervisors: Prof.dr. Y. Temel / Prof.dr. H.W.M. Steinbusch / Prof.dr. T. Sharp, Oxford, UK / Prof.dr. V. Visser-Vandewalle, Koln. 
Koen van Boxem: The use of pulsed radiofrequency in the management of chronic lumbosacral radicular pain. Supervisors: Prof.dr. M. van Kleef / Prof.dr. E.A.J. Joosten; Co-Supervisor: Assoc. Prof.dr. J. van Zundert.

Jérôme Waterval: Hyperostosis cranialis interna. Supervisors: Prof.dr. J.J. Manni / Prof.dr. R.J. Stokroos.

Sylvie Kolfschoten-van der Kruijs: Psychogenic non-epileptic seizures; the identification of neurophysiological correlates. Supervisors: Prof.dr. A.P. Aldenkamp / Prof.dr. K.E.J. Vonck, Universiteit Gent; Co-Supervisors: Dr. J.F.A. Jansen / Dr. R.H.C. Lazeron, Kempenhaeghe.

Wouter Pluijms: Spinal cord stimulation and pain relief in painful diabetic: polyneuropathy, a translational approach. Supervisors: Prof.dr. M. van Kleef / Prof.dr. E.A. Joosten; Co-supervisor: Dr. C.G. Faber.

Ron Handels: Health technology assessment of diagnostic strategies for Alzheimer's disease. Supervisors: Prof.dr. F.R.J. Verhey / Prof.dr. J.L. Severens (EUR); Co-Supervisor: Dr. M.A. Joore / Dr. C.A.G. Wolfs.

Evelyn Peelen: Regulatory T cells in the pathogenesis of Multiple Sclerosis: potential targets for vitamin D therapy. Supervisors: Prof.dr. R.M.M. Hupperts / Prof.dr. J.W. Cohen Tervaert; CoSupervisor: Dr. J.G.M.C. Damoiseaux / Dr. M.M.G.L.Thewissen, Diepenbeek.

Reint Jellema: Cell-based therapy for hypoxic-ischemic injury in the preterm brain. Supervisors: Prof.dr. B.W.W. Kramer / Prof.dr. H.W.M. Steinbusch; Co-Supervisor: Dr. W.T.V. Germeraad / Dr. P. Andriessen, Veldhoven.

Maria Wertli: Prognosis of Chronic Clinical Pain Conditions: The Example of Complex Regional Pain Syndrome 1 and Low Back Pain. Supervisors: Prof.dr. M. van Kleef; Co-Supervisor: Dr. F. Brunner, Zürich / Dr. R. Perez, VUmc.

Dagmar Zeef: An experimental model of Huntington's disease: Validation \& Stimulation. Supervisors: Prof.dr. Y. Temel / Prof.dr. H.W.M. Steinbusch; Co-supervisor: Dr. A. Jahanshahi.

Jeroen Decoster: Breaking Down Schizophrenia into phenes, genes and environment. Supervisors: Prof.dr. I. Myin-Germeys / Prof.dr. M. De Hert, KU Leuven; Co-Supervisor: Dr. R. van Winkel.

Eaja Anindya Sekhar Mukherjee: Fetal Alcohol Spectrum Disorders: exploring prevention and management. Supervisor: Prof.dr. L.M.G. Curfs; Co-Supervisor: Prof. S. Hollins, St. George's University of London, UK.

Catherine van Zelst: Inside out; On stereotype awareness, childhood trauma and stigma in psychosis. Supervisors: Prof.dr. Ph. Delespaul / Prof.dr. J. van Os.

Ibrahim Tolga Binbay: Extended Psychosis Phenotype in the Wider Social Environment. Supervisor: Prof.dr. J. van Os; Co-Supervisor: Dr. M. Drukker.

Frank Van Dael: OCD matters in psychosis. Supervisors: Prof.dr. J. van Os / Prof.dr. I. Myin-Germeys.

Pamela Kleikers: NOXious oxidative stress: from head toe too and back. Supervisors: Prof.dr. H.H.H.W. Schmidt / Prof.dr. H.W.M. Steinbusch; Co-Supervisor: Dr. B. Janssen.

José Luis Gerardo Nava: In vitro assay systems in the development of therapeutic interventions strategies for neuroprotection and repair. Supervisors: Prof.dr.med. J. Weis / Prof.dr. H.W.M. Steinbusch; Co-Supervisor: Dr. G.A. Brook, RWTH Aachen. 
Eva Bollen: Cyclic nucleotide signaling and plasticity. Supervisors: Prof.dr. H.W.M. Steinbusch / Prof.dr. R. D'Hooge, KU Leuven; Co-Supervisor: Dr. J. Prickaerts.

\section{5}

Jessica A. Hartmann: A good laugh and a long sleep; Insights from prospective and ambulatory assessments about the importance of positive affect and sleep in mental health. Supervisor: Prof.dr. J. van Os; Co-Supervisors: C.J.P. Simons / Dr. M. Wichers.

Bart Ament: Frailty in old age; conceptualization and care innovations. Supervisors: Prof.dr. G.I.J.M. Kempen / Prof.dr. F.R.J. Verhey; Co-Supervisor: Dr. M.E. de Vugt.

Mayke Janssens: Exploring course and outcome across the psychosis-continuum. Supervisor: Prof.dr. I. Myin-Germeys; Co-Supervisor: Dr. T. Lataster.

Dennis M.J. Hernau: Dopayours is not dopamine: genetic, environmental and pathological variations in dopaminergic stress processing. Supervisor: Prof.dr. I. MyinGermeys; Co-Supervisors: Prof.dr. F.M. Mottaghy / Dr. D. Collip.

Ingrid M.H. Brands: The adaptation process after acquired brain injury Pieces of the puzzle. Supervisors: Prof.dr. C.M. van Heugten / Prof.dr. D.T. Wade, Oxford UK; Co-Supervisors: Dr. S.Z. Stapert / Dr. S. Köhler.

Francesco Risso: Urinary and salivary S100B monitoring in high risk infants. Supervisor: Prof.dr. J.S.H. Vles; Co-Supervisors: Dr. D. Gazzolo, Genoa,Italy / Dr. A.W.D. Gavilanes.

Alessandro Borghesi: Stem and Progenitor Cells in Preterm Infants: Role in the Pathogenesis and Potential for Therapy. Supervisor: Prof.dr. L. Zimmermann; Prof.dr. B. Kramer; Co-Supervisors: Dr. D. Gazzolo, Genoa,Italy / Dr. A.W.D. Gavilanes.

Claudia Menne-Lothmann: Affect dynamics; A focus on genes, stress, and an opportunity for change. Supervisor: Prof.dr. J. van Os; Co-Supervisors: Dr. M. Wichers / Dr. N. Jacobs.

Martine van Nierop: Surviving childhood new perspectives on the link between childhood trauma and psychosis. Supervisors: Prof.dr. I. Myin-Germeys / Prof.dr. J. van Os; Co-Supervisor: Dr. R. van Winkel.

Sylvia Klinkenberg: VNS in children; more than just seizure reduction. Supervisors: Prof.dr. J. Vles / Prof.dr. A. Aldenkamp; Co-Supervisor: Dr. H. Majoie.

Anouk Linssen: Considerations in designing an adult hearing screening programme. Supervisor: Prof.dr. B. Kremer; Co-Supervisors: Dr. L. Anteunis / Dr. M. Joore.

Janny Hof: Hearing loss in young children; challenges in assessment and intervention. Supervisors: Prof.dr. B. Kremer / Prof.dr. R. Stokroos / Prof.dr. P. van Dijk, RUG; Co-Supervisor: Dr. L. Antheunis.

Kimberly Cox-Limpens: Mechanisms of endogenous brain protection; Clues from the transcriptome. Supervisors: Prof.dr. J. Vles / Prof.dr. L. Zimmermann; Co-Supervisor: Dr. A. Gavilanes.

Els Vanhoutte: Peripheral Neuropathy outcome measures; Standardisation (PeriNomS) study part 2: Getting consensus. Supervisors: Prof.dr. C. Faber / Prof.dr. P. van Doorn; Co-Supervisor: Dr. I. Merkies, Spaarne ziekenhuis Hoofddorp. 
Mayienne Bakkers: Small fibers, big troubles; diagnosis and implications of small fiber neuropathy. Supervisors: Prof.dr. C. Faber / Prof.dr. M. de Baets; Co-Supervisor: Dr. I. Merkies, Spaarne ziekenhuis Hoofddorp.

Ingrid Kramer: Zooming into the micro-level of experience: An approach for understanding and treating psychopathology. Supervisor: Prof.dr. J. van Os; Co-Supervisors: Dr. M. Wichers, UMC Groningen / Dr. C. Simons.

Esther Bouman: Risks and Benefits of Regional Anesthesia in the Perioperative Setting. Supervisors: Prof.dr. M. van Kleef / Prof.dr. M. Marcus, HMC, Qatar / Prof.dr. E. Joosten; Co-Supervisor: Dr. H. Gramke.

Mark Janssen: Selective stimulation of the subthalamic nucleus in Parkinson's disease; dream or near future. Supervisors: Prof.dr. Y. Temel / Prof.dr. V. Visser-Vandewalle, Keulen / Prof.dr. A. Benazzouz, Bordeax, France.

Reina de Kinderen: Health Technology Assessment in Epilepsy; economic evaluations and preference studies. Supervisors: Prof.dr. S. Evers / Prof.dr. A. Aldenkamp; Co-Supervisor: Dr. H. Majoie / Dr. D. Postulart, GGZ O-Brabant.

Saskia Ebus: Interictal epileptiform activity as a marker for clinical outcome. Supervisors: Prof.dr. A. Aldenkamp / Prof.dr. J. Arends, TUE / Prof.dr. P. Boon, Universiteit Gent, België.

Inge Knuts: Experimental and clinical studies into determinants of panic severity. Supervisor: Prof.dr. I. Myin-Germeys; Co-Supervisor: Dr. K. Schruers; Influencing panic.

Nienke Tielemans: Proactive coping post stroke: The Restored4Stroke Self-Management study. Supervisors: Prof.dr. C. van Heugten / Prof.dr. J. Visser-Meily, UMC Utrecht; Co-Supervisor: Dr. V. Schepers, UMC Utrecht.

Tom van Zundert: Improvements Towards Safer Extraglottic Airway Devices. Supervisors: Prof.dr. A.E.M. Marcus / Prof.dr. W. Buhre / Prof.dr. J.R. Brimacombe, Queensland, Australia / Prof.dr. C.A. Hagberg.

Tijmen van Assen: Anterior Cutaneous Nerve Entrapment Syndrome Epidemiology and surgical management. Supervisors: Prof.dr. G.L. Beets / Prof.dr. M. van Kleef / Dr. R.M.H. Roumen / Dr. M.R.M. Scheltinga, MMC Veldhoven.

Rohit Shetty: Understanding the Clinical, Immunological and Genetic Molecular Mechanisms of Keratoconus. Supervisors: Prof.dr. R.M.M.A. Nuijts / Prof.dr. C.A.B. Webers.

Christine van der Leeuw: Blood, bones and brains; peripheral biological endophenotypes and their structural cerebral correlates in psychotic disorder. Supervisor: Prof.dr. J. van Os; Co-supervisor: Dr. M. Marcelis.

Sanne Peeters: The Idle Mind Never Rests; functional brain connectivity across the psychosis continuum. Supervisor: Prof.dr. J. van Os; Co-supervisor: dr. M. Marcelis.

Nick van Goethem: $\alpha 7$ nicotinic acetylcholine receptors and memory processes: mechanistic and behavioral studies. Supervisor: Prof.dr. H.W.M. Steinbusch; Cosupervisor: Dr. J. Prickaerts.

Nicole Leibold: A Breath of fear; a translational approach into the mechanisms of panic.

Supervisor: Prof.dr. H.W.M. Steinbusch; Co-supervisors: Dr. K.R.J. Schruers / Dr. D.L.A. van den Hove. 
Renske Hamel: The course of mild cognitive impairment and the role of comorbidity. Supervisor: Prof.dr. F.R.J. Verhey; Co-supervisors: Dr. I.H.G.B. Ramakers / Dr. P.J. Visser.

Lucia Speth: Effects of botulinum toxin A injections and bimanual task-oriented therapy on hand functions and bimanual activities in unilateral Cerebral Palsy. Supervisors: Prof.dr. J. Vles; Prof.dr. R. Smeets; Co-supervisor: Dr. Y. Janssen-Potten, Adelante Hoensbroek.

Yuan Tian: The effects of Lutein on the inflammatory pathways in age-related macular degeneration (AMD). Supervisors: Prof.dr. C. Webers; Prof.dr. A. Kijlstra, WUR; Cosupervisor: Dr. M. Spreeuwenberg; Dr. H. Tange.

Peggy Spauwen: Cognition and Type 2 diabetes; the interplay of risk factors. Supervisors: Prof.dr. F. Verhey; Prof.dr. C. Stehouwer; Co-supervisor: Dr. M. van Boxtel

Marc Hilhorst: Crescentic glomerulonephritis in ANCA associated vasculitis. Supervisors: Prof.dr. J. Cohen-Tervaert; Co-supervisor: Dr. P. van Paassen Martin Gevonden: The odd one out: exploring the nature of the association between minority status and psychosis. Supervisors: Prof.dr. J-P. Selten; Prof.dr. J. Booij, Uva; Prof.dr. I. Myin-Germeys

Bart Biallosterski: Structural and functional aspects of sensory-motor Interaction in the urinary bladder. Supervisors: Prof.dr. Ph. Van Kerrebroeck; Prof.dr. S. De Wachter, UvAntwerpen; Cosupervisors: Dr. G. van Koeveringe; Dr. M. Rahnama'i.

Alexandra König: The use of information and communication technologies (ICT) for the assessment of patients with Alzheimer's Disease and related disorders. Supervisors: prof.dr. F. Verhey; prof.dr. Ph. Robert, Nice, Fr; Co-supervisors: dr. P. Aalten; dr. R. David, Nice. Fr.

Michelene Chenault: Assessing Readiness for Hearing Rehabilitation. Supervisors: prof.dr. M.P.F. Berger; prof.dr. B. Kremer; Co-supervisor: dr. L.J.C. Anteunis.

Anand Vinekar: Retinopathy of Prematurity. Recent advances in tele-medicine screening, risk factors and spectral domain optical coherence tomography imaging. Supervisor: prof.dr. C.A.B. Webers; Co-supervisor: dr. N.J. Bauer

Fleur van Dooren: Diabetes and Depression: exploring the Interface between Pathophysiological and Psychological factors. Supervisors: prof.dr. F.R.J. Verhey; prof.dr. J.K.L. Denollet, UvT; prof.dr. F. Pouwer, UvT; Co-supervisor: dr. M.T. Schram.

Gabriëlla Pons van Dijk: Taekwondo and physical fitness components in middle-aged healthy volunteers; the Sekwondo study. Supervisors: prof.dr. J. Lodder; prof.dr. H. Kingma; Co-supervisor: dr. A.F. Lenssen.

Yara Pujol López: Development and psychoneuroimmunological mechanisms in depression. Supervisor: prof.dr. H.W.M. Steinbusch; Co-supervisors: Dr. G. Kenis; Dr. D. van den Hove; Dr. Aye Mu Myint, München.

Romina Gentier: UBB ${ }^{+1}$; an important switch in the onset of Alzheimer's disease. Supervisors: Prof. H. Steinbusch; Prof. D. Hopkins; Co-supervisor: Dr. F. van Leeuwen.

Sanne Smeets: Insights into insight: studies on awareness of deficits after acquired brain injury. Supervisor: Prof. C. van Heugten; Prof. R. Ponds; Co-supervisor: Dr. I. Winkens

Kim Beerhorst: Bone disease in chronic epilepsy: fit for a fracture. Supervisor: Prof. A. Aldenkamp; Prof. R. van Oostenbrugge; Co-supervisor: Dr. P. Verschuure.

Alex Zwanenburg: Cerebral and cardiac signal monitoring in fetal sheep with hypoxicischemic encephalopathy. Supervisor: Prof. T. Delhaas; Prof. B. Kramer; Co-supervisors: Dr. T. Wolfs; Dr. P. Andriessen, MMC. 
Ismail Sinan Guloksuz: Biological mechanisms of environmental stressors in psychiatry. Supervisor: Prof. J. van Os; Co-supervisors: Dr. B. Rutten; Dr. M. Drukker.

Seyed Ehsan Pishva MD: Environmental Epigenetics in mental health and illness. Supervisor: Prof.dr. J. van Os; Co-supervisors: Dr. B.P.F. Rutten; Dr. G. Kenis.

Ankie Hamaekers: Rescue ventilation using expiratory ventilation assistance; innovating while clutching at straws. Supervisors: Prof.dr. W.F. Buhre; Prof.dr. M. van Kleef.

Rens Evers. 22q11.2 deletion syndrome: intelligence, psychopathology and neurochemistry at adult age. Supervisors: Prof.dr. L.M.G. Curfs; Prof.dr. T. v. Amelsvoort.

Sarah-Anna Hescham. Novel insights towards memory restoration. Supervisor: Prof.dr. Y. Temel; Co-supervisor: Dr. A. Blokland; Dr. A. Jahanshahi.

João P. da Costa Alvares Viegas Nunes. Insulin receptor sensitization improves affective pathology in various mouse models. Supervisor: Prof.dr. H.W.M. Steinbusch; Cosupervisors: Dr. KP. Lesch; Dr. T. Strekalova; Dr.B.H. Cline, Oxford.

Yanny Ying-Yee Cheng. Clinical Outcomes After Innovative Lamellar Corneal Transplantation Surgery. Supervisor: Prof.dr. R.M.M.A. Nuijts; Co-supervisor: Dr. J.S.A.G. Schouten.

\section{6}

Oliver Gerlach. Parkinson's disease, deterioration during hospitalization. Supervisor: Prof.dr. R. van Oostenbrugge; Co-supervisor: Dr. W. Weber.

Remo Arts. Intracochlear electrical stimulation to suppress tinnitus. Supervisor: Prof.dr. R.J. Stokroos; Co-supervisor: Dr. E.L.J. Georg.

Mitchel van Eeden. The $€$ - Restore4stroke study: Economic evaluation of stroke care in the Nethrelands. Supervisors: prof.dr. mr. S.M.A.A. Evers; prof.dr. C.M.v. Heugten; Co-supervisor: dr. G.A.P. v. Mastrigt.

Pim Klarenbeek. Blood pressure and cerebral small vessel disease. Supervisor: Prof.dr. R.J. van Oostenbrugge; Co-supervisor: Dr. J. Staals.

Ramona Hohnen. Peripheral pharmacological targets to modify bladder contractility. Supervisor: Prof.dr. Ph.E.V. van Kerrebroeck; Co-supervisors: Dr. G.A. van Koeveringe; Dr. M.A. Sahnama'i; Dr. C. Meriaux.

Ersoy Kocabicak. Deep brain stimulation of the subthalamic nucleus: Clinical and scientific aspects. Supervisors: Prof.dr. Y. Temel; Prof.dr. K. van Overbeeke; Co-supervisor: Dr. A. Jahanshahi.

Sven Akkerman. Temporal aspects of cyclic messenger signaling in object recognition memory; a pharmalogical approach. Supervisor: Prof.dr. H.W.M. Steinbusch; Co-supervisors: dr. J. Prickaerts; dr. A. Blokland.

Anja Moonen. Emotion and Cognition in Parkinson's disease; etiology and neurobiological mechanisms. Supervisor: Prof.dr. F.R.J. Verhey; Co-supervisor: dr. A.F.G. Leentjens.

Anna Schüth. Three-dimensional bladder tissue morphology. Supervisors: Prof.dr. G.A. van Koeveringe; Prof.dr. M. v. Zandvoort, Aachen; Prof.dr. Ph. V. Kerrebroeck.

Elisabeth van der Ven. Ethnic minority position as risk indicator for autism- Spectrum and psychotic disorders. Supervisors: Prof.dr. J.P. Selten; Prof.dr. J. van Os.

Zuzana Kasanova. Environmental reactivity for better or worse; The impact of stress and reward on neurochemistry, affect and behavior across the psychosis continuum. Supervisor: Prof.dr. I. Myin-Germeys, KU Leuven/UM; Co-supervisor: dr. D. Collip. 
Danielle Lambrechts. Ketogenic diet therapies; treatment for children and adults with refractory epilepsy. Supervisors: Prof.dr. H.J.M. Majoie; Prof.dr. J.S.H. Vles; Prof.dr. A.P. Aldenkamp; Cosupervisor: dr. A.J.A. de Louw, Kempenhaghe, Heeze.

Frank van Bussel. Advanced MRI in diabetes; cerebral biomarkers of cognitive decrements. Supervisors: Prof.dr.ir. W.H. Backes; Prof.dr. P.A.M. Hofman; Co-supervisor: dr. J.F.A. Jansen.

Lisa Schönfeldt. Neurostimulation to treat brain injury? Supervisors: Prof.dr. Y. Temel; Prof.dr. S. Hendrikx, Hasselt; Co-supervisor: dr. A. Jahanshahi.

Rianne Geerlings. Transition in patients with childhood-onset epilepsy; a long way to adulthood. Supervisor: Prof.dr. A.P. Aldenkamp; Co-supervisors:dr. A.J.A. de Louw, dr. L.M.C. Gottmer, Kempenhaeghe.

Nele Claes. B cells as multifactorial players in multiple sclerosis pathogenesis: insights from therapeutics. Supervisors: Prof.dr. V. Somers, Hasselt; Prof.dr. R. Hupperts Co-supervisors: Prof.dr. P. Stinissen, dr. J. Fraussen, Hasselt.

Olaf Schijns. Epilepsy surgery and biomarkers from history to molecular imaging. Supervisors: Prof.dr. J.J. van Overbeeke; Prof.dr. H. Clustermann, Aachen; Co-supervisors: dr. G. Hoogland; dr. M.J.P. v. Kroonenburgh.

Lizzy Boots. Balanced and Prepared; development and evaluation of a supportive ehealth intervention for caregivers of people with early-stage dementia. Supervisors: Prof.dr. F.R.J. Verhey; Prof.dr. G.I.J.M. Kempen; Co-supervisor: dr. M.E. de Vugt.

Wouter Donders. Towards patient-specific (cerebro-) vascular model applications. Supervisors: Prof.dr. T. Delhaas; Prof.dr.ir. F.N. van de Vosse, TUE; Co-supervisor: dr.ir. W. Huberts.

Sizzle Vanterpool. The implications of intrauterine invasion by microbes for placental Pathology and the occurrence of adverse pregnancy outcomes. Supervisor: Prof.dr. B.W. Kramer. Cosupervisors: dr. J.V. Been, Erasmus MC Rotterdam, dr. U von Rango.

Manuela Heins. The Relationship between Social Adversity, Psychosis, and Depression across an Individual's Life Span. Supervisor: Prof.dr. I. Myin-Germeys.

Christianus van Ganzewinkel. NEONATAL PAIN; Out of Sight, Out of Mind? Supervisor: Prof.dr. B.W.W. Kramer; Co-supervisor: dr. P. Andriessen, MMC Veldhoven.

Anne-Hilde Muris. Hype or hope? Vitamin D in multiple sclerosis; A clinical and immunological perspective. Supervisor: Prof.dr. R.M.M. Hupperts; Co-supervisor: dr. J.G.M.C. Damoiseaux.

Gerard Bode. The link between ceramide transporters, innate Immunity and Alzheimer's disease. Supervisor: Prof.dr. M.H.V. de Baets; Co-supervisors: dr. P. Martinez, dr. M. Losen.

Jo Stevens. Advanced diagnostics and therapeutics for Alzheimer's disease. Supervisor: Prof.dr. M. de Baets; Co-supervisors: dr. M. Losen, dr. P. Martinez-Martinez.

Rosan Luijcks. Stress and pain in muscles and brain; developing psychophysiological paradigms to examine stress and pain interactions. Supervisors: Prof.dr. J.J. van Os; Prof.dr.ir. H.J. Hermens, UT; Co-supervisor: dr. R. Lousberg.

M.C. Haanschoten. Towards efficient cardiac surgery - the integrating role of anesthesiology and intensive care. Supervisors: Prof. dr. W. Buhre; Prof. dr. A. van Zundert (Queensland); Co-supervisors: Dr. M.A. Soliman Hamad; Dr. A. van Straten (Catharina zkhs.)

Harmen Jan van de Haar. Microvascular and blood-brain barrier dysfunction in Alzheimer's disease. Supervisor: Prof.dr.ir. W. Backes; Prof.dr. F. Verhey; Co-supervisor: Dr. J. Jansen; Dr.ir. M. v. Osch, LUMC. 
Coenraad Itz. Chronic low back pain, considerations about: Natural Course, Diagnosis, Interventional Treatment and Costs. Supervisor: Prof.dr. M. van Kleef; Prof.dr. F. Huygen, EUR; Cosupervisor: Dr. B. Ramaekers.

Willemijn Jansen. The Path of Alzheimer's disease: from neuropathology to clinic. Supervisor: Prof.dr. F. Verhey; Co-supervisors: Dr. P.J. Visser; Dr. I. Ramakers.

Ligia dos Santos Mendes Lemes Soares. Phosphodiesterase inhibitors: a potential therapeutic approach for ischemic cerebral injury. Supervisor: Prof.dr. H.W.M. Steinbusch; Co-supervisors: Dr. R.M. Weffort de Oliveira, Brazil; Dr. J. Prickaerts

Martijn Broen. Anxiety and depression in Parkinson's disease. Supervisor: Prof.dr. R.J. van Oostenbrugge; Co-supervisors: Dr. A.F.G. Leentjens; Dr. M.L. Kuijf.

Sandra Schipper. Extrasynaptic receptors as a treatment target in epilepsy. Supervisor: Prof.dr. J.H.S. Vles; Co-supervisors: Dr. G. Hoogland; Dr. S. Klinkenberg; Dr. M.W. Aalbers, RUG.

João Casaca Carreira. Making sense of Antisense Oligonucleotides Therapy in Experimental Huntington's disease. Supervisor: Prof.dr. Y. Temel; Co-supervisors: Dr. A. Jahanshahi; Dr. W. van Roon-Mom, LUMC.

Dominique IJff. Trick or Treat? Cognitive side-effects of antiepileptic treatment. Supervisors: Prof.dr. A.P. Aldenkamp; Prof.dr. M. Majoie; Co-supervisors: Dr. J. Jansen; Dr. R. Lazeron, Kempenhaeghe.

Alfredo Ramirez. Neurogenetic approach in neurodegenerative disorders. Supervisors: Prof.dr. B.P.F. Rutten; Prof.dr. H.W.M. Steinbusch; Prof.dr. M.M. Nöthen, University of Bonn.

Nienke Visser. Toric Intraocular lenses in cataract surgery. Supervisor: Prof.dr. R.M.M.A. Nuijts; Cosupervisor: Dr. N.J.C. Bauer.

Jakob Burgstaller. Prognostic indicators for patients with degenerative lumbar spinal stenosis. Supervisor: Prof.dr. M. van Kleef; Co-supervisors: Dr. M.M. Wertli, University of Zurich; Dr. H.F. Gramke.

Mark van den Hurk. Neuronal Identity and Maturation: Insights from the Single-Cell Transcriptome. Supervisors: Prof.dr. H.W.M. Steinbusch; Prof.dr. B.P.F. Rutten; Cosupervisors: Dr. G. Kenis; Dr. C. Bardy, Adelaide.

Maria Nikiforou. Prenatal stress and the fetal gut. Potential interventions to prevent adverse outcomes. Supervisors: Prof.dr. B.W. Kramer; Prof.dr. H.W. Steinbusch; Cosupervisor: Dr. T.G. Wolfs.

Janneke Peijnenborgh. Assessment of cognition, time perception, and motivation in children. Supervisors: Prof.dr. J.S.H. Vles; Prof.dr. A.P. Aldenkamp; Co-supervisors: Dr. J. Hendriksen; Dr. P. Hurks.

Joany Millenaar. Young onset dementia; towards a better understanding of care needs and experiences. Supervisors: Prof.dr. F. Verhey; Prof.dr. R. Koopmans, RUN; Cosupervisors: Dr. M. de Vugt; Dr. C. Bakker, RUN.

\section{7}

Adriana Smits. Perinatal factors and hearing outcome. Supervisors: Prof.dr. R.J. Stokroos; Prof.dr. B.W. Kramer; Prof.dr. B. Kremer.

Angela Bouwmans. Transcranial sonography in parkinsonian disorders: clear window or blurred vision. Supervisor: Prof.dr. W.H. Mess; Co-promotores: Dr. W.E.J. Weber; Dr. A.F.G. Leentjens.

Björn K. Stessel. Patient centred care after day surgery: scope for improvement. Supervisors: Prof.dr. W. Buhre; Prof.dr. B. Joosten. Co-supervisor: Dr. A.H. Gramke. 
Jan Guy Bogaarts. Quantitative EEG and machine learning methods for the detection of epileptic seizures and cerebral asymmetry. Supervisor: Prof.dr. W.M. Mess; Co-supervisor: Dr.ir. J.P.H. Reulen; Dr.ir. E.D. Gommer.

Martin M. Müller. Pregnancy derived products for treatment of perinatal brain injuries. Supervisors: Prof.dr. B.W.W. Kramer; Prof.dr. D. Surbek, Bern; Co-supervisors: Dr. T. Wolfs; Dr. G. Gavilanes.

Daan Ophelders. Novel treatment strategies for the protection of the preterm brain; Rebalancing inflammation and regeneration. Supervisor: Prof.dr. B. Kramer; Co-supervisor: Dr. T. Wolfs; Dr. R. Jellema.

Rosalie van Knippenberg. Experience sampling in dementia care; an innovative intervention to support caregivers in daily life. Supervisors: Prof.dr. F. Verhey; Prof.dr. R. Ponds; Prof.dr. I. MyinGermeys, KU Leuven; Co-supervisor: Dr. M. de Vugt.

Claudia Vingerhoets. Investigating neurobiological mechanisms underlying comorbid cognitive symptoms in psychosis and substance use. Supervisors: Prof.dr. T. van Amelsvoort; Prof.dr. J. Booij, UvA; Co-supervisor: Dr. O. Bloemen

Dennis Oerlemans. Evolution of Neuromodulation for Lower Urinary Tract Dysfunction; Past, Present and Future. Supervisors: Prof.dr. Ph. van Kerrebroeck; Prof.dr. G. van Koeveringe. Cosupervisors: Dr. E. Weil; Dr. T. Marcelissen.

Marion Levy. Evaluation of BDNF/TrkB signaling as a common target in the treatment of major depression and Alzheimer's disease. Supervisors: Prof.dr. H. Steinbusch; Prof. L. Lanfumey, Université Paris Descartes, France. Co-supervisors: Dr. G. Kenis; Dr. D. van den Hove.

Patrick Domen. Stay connected: a family-based diffusion imaging study in psychotic disorder. Supervisor: Prof.dr. J. van Os. Co-supervisor: Dr. M. Marcelis

Geor Bakker. Innovative Approaches to Understanding the Neurobiology of Psychosis. Supervisors: Prof.dr. T. van Amelsfoort; Prof.dr. J. Booij, UvA. Co-supervisor: dr. M. Caan, UvA; dr. O. Bloemen.

Wilma Boevink. HEE! Over Herstel, Empowerment en Ervaringsdeskundigheid in de psychiatrie. Supervisors: Prof.dr. J. van Os; Prof.dr. Ph. Delespaul. Co-supervisor: dr. H. Kroon.

Nataliia Markova . Modified swim test as a mouse depression paradigm of enhanced Cognitive processing: the role of GSK3 $\beta$. Supervisor: Prof.dr. H. Steinbusch; Prof.dr. K-P. Lesch, University of Wuerzburg. Co-supervisor: Dr. T. Strekalova.

Merijn van de Laar. Individual differences in insomnia; implications of Psychological factors for diagnosis and treatment. Supervisor: Prof.dr. A. Aldenkamp; Prof.dr. D. Pevernagie, Universiteit Gent. Co-supervisor: Dr. S. Overeem, TUE.

Willem Buskermolen. If only I could tell ...; Measuring predictors for challenging behaviour in people with both intellectual disability and hearing impairment. Supervisor: Prof.dr. A. Aldenkamp. Co-supervisor: Dr. J. Hoekman, UL.

Kay Deckers. The role of lifestyle factors in primary prevention of dementia; an epidemiological perspective. Supervisor: Prof.dr. F. Verhey. Co-supervisor: Dr. M. van Boxtel; Dr. S. Köhler.

Brechje Dandachi-FitzGerald. Symptom validity in clinical assessments. Supervisors: Prof.dr. R. Ponds; Prof.dr. F. Verhey.

Maurice Theunissen. Understanding factors affecting postoperative Quality of Life. Supervisors: Prof.dr. M. Peters, Prof.dr. M. Marcus. Co-supervisor: Dr. H. Gramke. 
Anna Cleutjens. COgnitive-Pulmonary Disease? Neuropsychological functioning in patients with COPD. Supervisors: Prof.dr. E. Wouters, Prof.dr. R. Ponds. Co-supervisors: Dr. D. Janssen, Horn, Dr. J. Dijkstra.

Laura Serpero. Next Generaton Biomarkers in Perinatal Medicine: S100B Protein. Supervisors: Prof.dr. D. Gazzalo, Alessandria, Italy; Prof.dr. B..W.W. Kramer. Co-supervisor: Dr. A.W.D. Gavilanes.

Alessandro Varrica. S100B Protein and Congential Heart Diseases: Brain Aspects. Supervisors: Prof.dr. D. Gazzalo, Alessandria, Italy; Prof.dr. J.S.H. Vles; Prof.dr. L.J.I. Zimmermann. Co-supervisor: Dr. A.W.D. Gavilanes.

Pim R.A. Heckman. Targeting phosphodiesterase type $\mathbf{4}$ for improving cognitive frontostriatal function: a translational approach. Supervisor: Prof.dr. J.G. Ramaekers. Cosupervisors: Dr. J.H.H.J.. Prickaerts; Dr. A. Blokland.

Sven van Poucke. Platelets, form sample to big data; exploring granularity in platelet research. Supervisors: Prof.dr. M.A.E. Marcus; Prof.dr. W. Buhre. Co-supervisor: Dr. M. Lancé.

Désirée M.J. Vrijens. Dysfunctions of the Lower Urinary Tract and Affective Symptoms. Supervisors: Prof.dr. Ph.E.V. van Kerrebroeck; Prof.dr. G.A. van Koeveringe. Co-supervisors: Dr. C. Leue.

Tamar van Veenendaal. Neurotransmitters \& Networks. An MR view on epilepsy and antiepileptic drugs. Supervisors: Prof.dr.ir. W.H. Backes; Prof.dr. A.P. Aldenkamp. Cosupervisor: Dr. J.F.A. Jansen.

Evelien M. Barendse. Autism Spectrum Disorders in High functioning Adolescents; Diagnostic considerations (AHA). Supervisors: Prof.dr. A.P. Aldenkamp; Prof.dr. R.P.C. Kessels, Radboud University.

Roy Lardenoije. A venture into the epigenetics of aging and Alzheimer's Disease. Supervisors: Prof.dr. B.P.F. Rutten; Prof.dr. H.W.M. Steinbusch. Co-supervisors: Dr. D. van den Hove; Dr. C.A. Lemere, USA.

Charlotte L. Mentzel. The course recognition and treatment of movement disorders in severe mental illness. Supervisors: Prof.dr. P.N. van Harten; Prof.dr. M.A.J. de KoningTijssen, UMCG. Cosupervisor: Dr. P.R. Bakker.

Tim Batink. Third Wave Behaviour Therapy: Process Measures and Contextual Interventions. Supervisors: Prof.dr. F.P.M.L. Peeters; Prof.dr. J.J. van Os; Prof.dr. M.C. Wichers, UMC Groningen.

Kevin L.J. Rademakers. Detrusor Underactivity: From Theory To Clinical Assessment. Supervisors: Prof.dr. G.A. van Koeveringe; Prof.dr. Ph.E.V. van Kerrebroeck. Co-supervisor: Dr. M. Oelke.

Iris M.J. Lange. Should I stay or should I go ? Brain mechanisms underlying fear and safety learning, and explosure therapy outcome. Supervisors: Prof.dr. K.R.J. Schruers; Prof.dr. T.A.M.J. van Amelsfoort. Co-supervisor: Dr. L. Goossens.

Ruben G.F. Hendriksen. Evidence for a dystrophin-associated encephalopathy in Duchenne Muscular Dystrophy. Supervisor: Prof.dr. J.S.H. Vles. Co-supervisors: Dr. G. Hoogland; Dr. M.W. Aalbers, UMC Groningen.

Michael Gofeld. Strengths and limitations of the lumbar spine ultrasound-guided interventions. Supervisor: Prof.dr. M. van Kleef. Co-supervisor: Dr. M. Sommer.

Willem A.R. Zwaans. Strategies for chronic inguinal pain. Supervisor: Prof.dr. M. van Kleef. Cosupervisors: Dr. R.H.M. Roumen; Dr. M.R.M. Scheltinga, MMC Veldhoven.

Linda M. Rolf. Mapping the effects of vitamin D in multiple sclerosis A 3D Perspective. Supervisor: Prof.dr. R.M.M. Hupperts. Co-supervisors: Dr. J.G.M.C. Damoiseaux; Dr. J.J.F.M. Smolders, CWZ Nijmegen. 
Maarten van Beek. Spinal Cord Stimulation in Clinical and Experimental Painful Diabetic Polyneuropathy. Supervisors: Prof.dr. E.A. Joosten; Prof.dr. M. van Kleef. Cosupervisor: Dr. S.M.J. van Kuijk.

Melina Barkhuizen. Genetic and perinatal risk factors for movement disorders. Supervisors: prof.dr. B.W.W. Kramer, prof.dr. H.W.M. Steinbusch, Prof.dr. A.F. Grobler. Cosupervisor: dr. A.W.D.GavilanesJimenez.

Renske Uiterwijk. Cognitive function and cerebral small vessel disease in hypertension. Supervisor: prof.dr. R.J. van Oostenbrugge. Co-supervisor: Dr. J.E.A. Staals.

Elles Douven. Depression and apathy after stroke. Supervisor: prof.dr. F.R.J. Verhey. Cosupervisors: Dr. P. Aalten, dr. J. Staals.

Mauro Pessia. Brain K+ Channels: from molecular and physiological features to autism spectrum disorder and intellectual disability. Supervisors: prof.dr. H.W.M. Steinbusch, prof.dr. M.B. Donati, It.

Carsten Leue. Hyperarousal in the Hospital and what to do about it: the MED-PSYCHNET - a transitional network approach fostering personalized care in psychosomatic medicine. Supervisors: Prof.dr. J. van Os, Prof.dr. A. Masclee. Co-supervisors: Dr. J. Strik, Dr. J. Kruimel

Andrea S. Herrera Soto. Aminochrome, an endotoxin for inducing a new rat model of Parkinson's Disease. Supervisor: prof.dr. H.W.M. Steinbusch. Co-supervisors: Prof.dr. Juan Segura-Aquilar; prof. G. Diaz-Veliz, Santiago of Chile

Eline E.B. de Clerck. Ocular neurodegenerative changes and macular cysts in prediabetes and type 2 diabetes. Supervisors: Prof.dr. C.A.B. Webers, Prof.dr. C.D.A. Stehouwer. Co-supervisor: Dr. J.S.A.G. Schouten

Steven T.H. Honings. Exploring psychosis and multidirectional violence: a prospective study in the general population. Supervisor: Prof.dr. J. van Os. Co-supervisor: Dr. M. Drukker

\section{8}

Sau May Wong. Advances in Microvasculair MRI Techniques: Breaking the Pathophysiological Barriers in Cerebral Small Vessel Disease. Supervisor: Prof.drir. W.H. Backes, Porf.dr. R.J. van Oostenbrugge. Co-supervisor: Dr. J.F.A. Jansen

Mark B.N. van Winkel. Lonely at heart and stressed in company of Others; the influence of daily life social experiences and emotions on depression. Supervisors: prof.dr. F. Peeters; prof.dr. I. MyinGermeys, KU Leuven/UM; prof.dr. M. Wichers, UMC Groningen

Harsha Birur Laxmana Rao. Revisiting the vascular theory of glaucoma using optical coherence tomography angiography. Supervisors: prof.dr. C.A.B. Webers; prof.dr. R.N. Weinreb, University of California, San Diego

Babette L.R. Reijs. Cognitive correlates of cerebrospinal fluid biomarkers for Alzheimer's disease. Supervisor: prof.dr. F.R.J. Verhey. Co-supervisors: Dr. P.J. Visser; dr. I.H.G.B. Ramakers

Rachel Slangen. Spinal cord stimulation in painful diabetic peripheral Neuropathy. Clinical- and cost-effectiveness. Supervisors: prof.dr. M. van Kleef; Prof.dr. C. Dirksen; prof.dr. C. Faber

Ganne Chaitanya. Epilepsy: A network disorder. Supervisors: prof.dr. A.P. Aldenkamp; prof. P. Satishchandra, NIMHANS, Bangalore, India. Co-supervisors: Dr. J.F.A. Jansen; Dr. S. Zinger, TUE

Sumitha Rajendrarao. New Insight into the Multifaceted Pathogenic Mechanisms of Sporadic Amyotrophic Lateral Sclerosis. Supervisors: prof.dr. B.W. Kamer; prof.dr. H.W. Steinbusch. Cosupervisor: prof. T.R. Raju, NIMHANS, Bangalore, India

Suzanne Roggeveen. Interference of mobile phone with electrophysiology and emotions; results from short-term experimental studies. Supervisor: Prof.dr. J. van Os. Co-supervisor: Dr. R. Lousberg. 
Matthias Walter. Multi-methodological approaches to investigate lower urinary tract function in health and disease. Supervisors: Prof.dr. Ph.E.V.A. van Kerrebroek; Prof.dr. G.A. van Koeveringe; Prof.dr. A. Curt, Zürich, CH.

Lalit Gupta. Inhomogeneities in spontaneous brain fluctuations. Supervisors: Prof.dr.ir. WH. Backes; Prof.dr. P.A.M. Hofman. Co-supervisor: Dr. J.F.A. Jansen.

Chaitra Jayadev. Impact of imaging the pediatric retina. Supervisor: Prof.dr. C.A.B. Webers. Cosupervisor: Dr. N.J.C. Bauer; Dr. A. Vinekar.

Annelie Klippel. Navigating through complexity; processes and mechanisms underlying the development of psychosis. Supervisors: Prof.dr. I. Myin-Germeys, KU-Leuven; Prof.dr. M.C. Wichers, UMC Groningen. Co-supervisor: Dr. U. Reininghaus.

Kürşat Altinbaş. Reconstructing The Diagnostic Framework of Bipolarity. Supervisor: Prof.dr. J. van Os. Co-supervisor: Dr. I.S. Gülöksüz.

Andrea J.R. Balthasar. Eyes of the needle; Spectral tissue sensing, an innovative technology for detecting various tissue types during percutaneous needle-based procedures in locoregional anesthesia and pain medicine. Supervisor: Prof.dr. M. van Kleef. Co-supervisor: Dr. G-J. van Geffen, Radboud UMC Nijmegen.

Walmari Pilz. Shedding light on oropharyngeal dysphagia in myotonic dystrophy type 1. Supervisor: Prof.dr. B. Kremer. Co-supervisors: Dr. L.W.J. Baijens; Dr. V. Lima Passos.

Nynke J. van den Hoogen. Repetitive painful procedures in the neonate: Treatment and adult pain sensitivity. Supervisors: Prof.dr. E.A.J. Joosten, Prof.dr. D. Tibboel, Erasmus MC-Sophia, Rotterdam. Co-supervisor: Dr. J. Patijn.

Carlota Mestres Gonzalvo. Medication optimisation; Methodological aspects and new strategies. Supervisors: Prof.dr. F.R.J. Verhey, Prof.dr. P.H.M. van der Kuy, Erasmus MC Rotterdam. Cosupervisors: Dr. R. Janknegt, Zuyderland MC.

Carolin Hoffmann. The Brain under Attack: Autoantibodies in Psychotic Disorders. Supervisors: Prof.dr. P. Martinez, Prof.dr. B. Rutten, Prof.dr. J. van Os, UU/UM.

Jindra M. Bakker. On the bumpy road of happiness: Mechanisms of daily life reward processing and how it can be changed. Supervisors: Prof.dr. M. Wichers, UMC Groningen, Prof.dr. I. MyinGermeys, KU Leuven/UM. Co-supervisor: Dr. L. Goossens.

Marasha-Fiona de Jong. Between mood and matter; studies on the interface between mood disorders and physical conditions. Supervisor: Prof.dr. F.P.M.L. Peeters. Cosupervisors: Prof.dr. Mischoulon.

Anouk Smeets. New insights in deep brain stimulation for Tourette syndrome. Supervisor: Prof.dr. Y. Temel. Co-supervisors: Dr. L. Ackermans, Dr. A.A. Duits, de. A.F.G. Leentjens.

Margaretha Skowron. Cisplatin resistance in urothelial carcinoma; Understanding and targeting inherent and acquired mechanisms. Supervisors: Prof.dr. G.A. van Koeveringe, Prof.dr. P. Albers, Heinrich-Heine Univ. Düsseldorf. Co-supervisors: Dr. J.G.H. van Roermund, Dr. A. Romano.

Thierry Mentzel. Capturing the cacophony of movement. Supervisors: Prof.dr. P.N. van Harten, Prof.dr. H.A.M. Daanen, VUA. Co-supervisor: Dr.mr. O.J.N. Bloemen, GGZ Hilversum/UM.

Petronella de Meij. Quality indicators for the assessment of pain clinic care: A step forward? Quality from professionals and pain patients' perspective (QiPPP). Supervisors: Prof.dr. G.D.E.M. van der Weijden, Prof.dr. M. v. Kleef. Co-supervisor: Dr. A.J.A. Köke.

Thomas Vaessen. Stress sensitivity in psychosis: assessment, mechanism \& intervention. Supervisor: Prof.dr. I. Myin-Germeys, KU Leuven/UM. 
Yori van der Steen. Dissecting the psychosis continuum; risk factors along the pathway from experiences to disorder. Supervisor: Prof.dr. I. Myin-Germeys, KU Leuven/UM, Prof.dr. R. van Winkel, KU Leuven.

Aryo Zare. Unveiling the sensory connections between the bladder and the brain that involve the periaqueductal gray matter. Supervisor: Prof.dr. G.A. van Koeveringe; Cosupervisor: Dr. A. Jahanshahi.

Magdalena Weidner. Brain serotonin throughout development - for better and for worse. Supervisors: Prof.dr. H.W.M. Steinbusch, Prof.dr. K.P. Lesch, JM.Univ. Würzburg. Cosupervisor: Dr. D.L.A. van den Hove.

Catherine Vossen. Cortical processing of pain; the role of habituation. Supervisors: Prof.dr. E.A. Joosten, Prof.dr. J. van Os, UU/UM. Co-supervisor: Dr. R. Lousberg.

Whitney Freeze. Microvascular contributions to dementia; Exploring the role of bloodbrain barrier leakage in cerebral small vessel disease and Alzheimer disease. Supervisors: Prof.dr. F.R.J. Verhey, Prof.dr.ir. W.H. Backes. Co-supervisor: Dr. H.I.L. Jacobs.

Simone Schüller. Characterization of Stem and Immune Cell Ontogeny to Inform Prevention and Treatment of Infections in Preterm Newborns. Supervisors: Prof.dr. B.W.W. Kramer, Prof.dr.med. A. Berger, Wien. Co-supervisor: Dr. E. Villamor.

Michael J. Kemna. Predicting relapses in ANCA associated vasculitis. Supervisor: Prof.dr. J.W. Cohen Tervaert. Co-supervisors: Dr. J. Damoiseaux, Dr. P. van Paassen.

Artemis latrou. Epigenetics in mental and neurodegenerative disorders. Supervisor: Prof.dr. B.P.F. Rutten. Co-supervisors: Dr. D.L.A. van den Hove, Dr. G. Kenis.

Laura Wielders. Prevention \& Treatment of Cystoid Macular Edema after Cataract Surgery. Supervisor: Prof.dr. R.M.M. Nuijts. Co-supervisors: Dr. J.S.A.G. Schouten, CWZ Nijmegen, Dr. B. Winkens.

Daisy Hoofwijk. The way to understanding Chronic Postsurgical Pain; From clinical and psychological predictors to incorporating genetics. Supervisor: Prof.dr. W.F.F.A. Buhre; Prof.dr. E.A.J. Joosten; Co-Supervisor: dr. H.-F. Gramke; dr. A.A.A. Fiddelers.

Loes Leenen. Self-management in Epilepsy; The Goal is: "Live with a Z(s)mile. Supervisors: Prof.dr. H.J.M. Majoie; Prof.dr.mr. S.M.A.A. Evers; Prof.dr. C.M. van Heugten.

Chiara Peila. 'Effects of Pasteurization and Refrigerated Storage on Human Milk Neurobiomarkers Concentrations. Supervisors: Prof.dr. D. Gazzallo, Alessandria, It./MUMC+; Prof.dr. G. Visser, UU; Prof.dr. E. Bertino, Alessandria, It.

Raymond van de Berg. The Vestibular Implant: Feasibility in humans. Supervisor: Prof.dr. H. Kingma; Co-supervisor: dr. J.-P. Guyot, Université de Genève, $\mathrm{CH}$.

Nils Guinand. The Vestibular Implant: a more stable horizon for patients with a bilateral vestibular deficit? Supervisors: prof.dr. H. Kingma; Prof.dr. J.-P. Guyot, Université de Genève, CH.

Jasper Smit. Exploring deep brain stimulation as a treatment for tinnitus. Supervisors: Prof.dr. R.J. Stokroos; Prof.dr. Y. Temel; Co-supervisor: dr. Jahanshahianvar.

Bindu Paravil Sankaran. Brain MRI in Mitochondrial Disorders: Correlating the Phenotype with Genotype. Supervisor: Prof.dr. H. Smeets; Prof.dr. A. Taly, NIMHANS, Bangalore, India.

Syenna Schievink. Vascular cognitive impairment; at the heart of the matter. Supervisor: Prof.dr. F.R.J. Verhey; Prof.dr. R.J. van Oostenbrugge; Co-supervisor: dr. S. Köhler. 
Isabelle Bos. Biomarkers of Alzheimer's disease; relations with vascular factors and cognition in the pre-dementia stages. Supervisor: Dr. P.J. Visser; Prof.dr. F.R.J. Verhey; Co-supervisor: dr. S.J.B. Vos.

Stijn Michielse. Road work ahead; cerebral pathways mediating Psychological mechanisms underlying the psychosis spectrum. Supervisor: Prof.dr. J.J. van Os; Cosupervisor: dr. M.C. Marcelis.

Georgios Schoretsanitis. Risperidone-based therapeutic regimens; Drug interactions and adverse drug reactions. Supervisor: prof.dr. K.R.J. Schruers; Co-supervisor: dr. M. Bak .

Alieske Dam. INLIFE; An innovative online social support intervention for caregivers of persons with dementia. Supervisor: Prof.dr. M.E. de Vugt; Prof.dr. F.R.J. Verhey; Cosupervisor: Dr. M.P.J. van Boxtel.

Roel Haeren. Vascular ventures; Analysis of vascular structures and function in epilepsy. Supervisor: Prof.dr. Y Temel; Co-supervisor: dr. K. Rijkers; Dr. G. Hoogland.

Chiara Fabbri. Pharmacogenomics of antidepressant drugs: perspectives for the personalization of treatment in depression. Supervisors: Prof.dr. K. Schruers; Prof.dr. A. Serretti, Bologna.

Esther van Duin. Dancing in the (B)rain'; neurobiology of reward, stress \& Information processing in 22q11.2 deletion syndrome. Supervisors: Prof.dr. T. van Amelsvoort; Prof.dr. J. Booij, UvA. Cosupervisor: dr. D. Hernaus.

Rob Verdonschot. Oropharyngeal dysphagia and its psychiatric Comorbidities; The prevalence of affective symptoms and the unmet clinical need for integrated care in medically unexplained symptoms. Supervisor: Prof.dr. B. Kremer; Co-supervisors; Dr. L. Baijens; dr. S. Vanbelle.

Lisanne Breuer. Accelerated Cognitive Ageing in Epilepsy' Does it Exists? Supervisors: Prof.dr. A. Aldenkamp; Prof.dr. P. Boon, UZ Gent; Co-supervisors: dr. A. de Louw, Kempenhaeghe, Heeze; dr.ir. S. Zinger, TUE.

Liselot Kerpershoek. Access to formal dementia care; A European perspective. Supervisors: Prof.dr. F. Verhey; Prof.dr. M. de Vugt; Prof. B. Woods, Bangor University, UK Co-supervisor: Dr. C. Wolfs.

Henrietta Steinhart. Same Same but Different; Psychological Interventions and how to Mind the Knowledge Practice Gap. Supervisor: Prof.dr. I. Myin-Germeys. Co-supervisor: Dr. U. Reininghaus.

Ulrich Mehnert. The management of urine storage dysfunction in the neurological patient. Supervisors: Prof.dr. G. van Koeveringe; Prof.dr. Ph.van Kerrebroeck; Prof.dr. S. Wachter, Antwerpen; Prof.dr E. Chartier-Kastler, Sorbonne, Paris.

Giovanne B. Diniz. Weaning-induced alterations on neuropeptidergic populations of the rat hypothalamus. Supervisors: Prof.dr. H. Steinbusch; Prof.dr. J. Bittencourt, ICB/USP, Brasil.

Rajani Ravindra Battu. Inherited Retinal Diseases: New Imaging and Molecular Genetics. Supervisor: Prof.dr. C.A.B. Webers. Co-supervisors:Dr. J.S.A.G. Schouten, CWZ; dr. T.T.J.M. Berendschot.

\section{9}

Jans van Ool. Diagnostic and neuropsychiatric considerations in epilepsy and intellectual disability; Psychological perspectives. Supervisor: Prof.dr. A. Aldenkamp. Co-supervisors: Dr. J. Hendriksen; Dr. H. Schelhaas, Kempenhaeghe.

Eveline Janssen. Depression in the elderly: focus on high risk groups. Supervisors: Prof.dr. F. Verhey; Prof.dr. M. de Vugt. Co-supervisor: Dr. M. Schram. 
Cécile Kicken. Extreme blood coagulation; investigating the influence of physiological extremes on thrombin generation and platelet activation. Supervisor: Prof.dr. W. Buhre Co-supervisors; Dr. B. de Laat; Dr. M. Lancé, Qatar.

Martinus van Eerd. Diagnosis and Interventional Pain Treatment of Cervical Facet Joint Pain. Supervisor: Prof.dr. M. van Kleef. Co-supervisor; Dr. J. Patijn, Eindhoven; Dr. M. Sommer.

Chenxing E. Zhang. Novel insights in the pathophysiology of cerebralsmall vessel disease - a study using advanced imaging techniques. Supervisors: Prof.dr. R.J. van Oostenbrugge; Prof.dr.ir. W.H. Backes; Co-supervisor: dr. J. Staals.

Ivo Eijkenboom. A zebrafish model of small-fiber neuropathy. Supervisors: Prof.dr. H.J.M. Smeets; Prof.dr. C.G. Faber; Co-supervisor: dr. J. Vanoevelen.

Bianca de Greef. Small fiber neuropathy: from underlying conditions to treatment. Supervisor: Prof.dr. C.A. Faber; Co-supervisor: Dr. I.S.J. Merkies; Dr. J.G.J. Hoeijmakers.

Lotte Berk. MINDFULNESS AND AGING: Exploring Mechanisms and Interventions. Supervisors: Prof.dr. J. van Os; Prof.dr. M.W. de Vugt; Co-supervisor: dr. M.P.J. van Boxtel.

Mor Dickman. Practice patterns and outcomes of corneal transplantation. Supervisor: Prof.dr. R.M.M.A. Nuijts; Co-supervisors: Dr. T.J.M. Berendschot; dr. F.J.H.M. van den Biggelaar.

Thyagi Ponnamperuma. Mental Health Problems in Sri Lankan Adolescents Exposed to the Tsunami and Other Traumatic Events. Supervisor: Prof.dr. M.W. De Vries; Co-supervisor: Dr. N.A. Nicolson.

Robbert C. Maatman. Anterior cutaneous nerve entrapment syndrome (acnes): an analysis of various subtypes and alternative treatment modalities. Supervisor: Prof.dr. M. van Kleef; Cosupervisors: Dr. R.M.H. Roumen, dr. M.R.M. Scheltinga.

Mari Elshout. Neovascular Age-Related Macular Degeneration in the Era of Value-Based Health Care. Supervisor: Prof.dr. C.A.B. Webers; Co-supervisor: Dr. J.S.A.G. Schouten.

Jeroen Deenik. Thinking inside the box; Changing lifestyle to improve the health status of inpatients with severe mental illness. Supervisor: Prof.dr. P.N. Harten; Co-supervisors: Dr. D.E. Tenback; dr. I.J.M. Hendriksen.

Thomas Draak. Peripheral Neuropathy outcome measures Standardisation (PeriNomS) study part 3: Capturing the Patient's Voice. Supervisor: Prof.dr. C.G. Faber; Co-supervisor: Dr. I.S.J. Merkies.

Ana Luisa Gil Martínez. Neuroprotection in neurodegenerative processes associated with Parkinsonism and aging. Correlation between dopaminergic neuronal death and glial activation. Supervisor: Prof.dr. H.W.M. Steinbusch, Prof.dr. Maria-Trinidad Herrero Ezquerro, University of Murcia.

Bernice J.A. Gulpers. Anxiety in older adults; Correlates, comorbidities and prognosis with lifespan perspectives. Supervisor: Prof.dr. F.R.J. Verhey, Prof.dr. R.C. Oude Voshaar; Co-supervisor: Dr. S. Köhler.

Elke Devocht. Combining a cochlear implant and a hearing aid in opposite ears: The best of both worlds. Supervisor: prof.dr. H. Kingma; co- supervisor: dr. E.I.J. George.

Gillian Townend. Rett Syndrome: Recognising the Communication Challenges, Needs and Potential of Individuals Living with a Rare Disease. Supervisor: Prof.dr. L.M.G. Curfs; co- supervisor: Dr. P.B. Marschik, Med. University of Graz, Austria.

Takashi Koizumi. Genetic and neuroinflammatory components of familial and sporadic cerebral Small Vessel Disease. Supervisor: Prof.dr. H. Steinbusch,Prof.dr. T. Mizuno,Japan; co-supervisor: Dr. S. Foulquier. 
Muhammad Ali. Integrative network-based approaches for modelling Human disease. Supervisor: Prof.dr. J. Kleinjans; co-supervisor: Dr. D. van den Hove; Dr. E. Pishva.

Guillaume Durand. The adaptive side of psychopathy. Investigating adaptive characteristics associated with the psychopathic personality. Supervisor: Prof.dr. B. Rutten; co-supervisor: Dr J. Lobbestael.

Darius C. Henatsch. Honey: A Novel Treatment in Chronic Ear Infections. Supervisor: Prof.dr. R.J. Stokroos; UMC Utrecht/UM; co-supervisor: Dr. J.J. Briedé.

Reinhilde J. Melles. Vaginal penetration: pain or pleasure? The role of fear and sexual arousal. Supervisor: Prof.dr. M.L. Peters; co-supervisor: Dr. M. ter Kuile, LUMC, Dr. M. Dewitte.

Raul Felipe Abella Antón. Cardiac Surgery Biochemical Monitoring in Congenital Heart Diseases Infants. Supervisors: Prof. dr. D. Gazzolo, Prof. dr. L.J.I. Zimmermann, Prof. dr. J.S.H. Vles, cosupervisor; Dr. A.W.D. Gavilanes.

Francesca M. Snoeijen-Schouwenaars. Diagnostic, neuropsychiatric and therapeutic considerations in epilepsy and intellectual disability - medical perspectives -. Supervisor: prof.dr. A.P. Aldenkamp, co-supervisors: Dr. H.J. Schelhaas, SEIN Zwolle; dr. J.G.M. Hendriksen, Kempenhaeghe, Heeze.

Mariëlle H.J. Pruppers. Peripheral Neuropathies: Standardizing Functional Assessment. Supervisors: prof.dr. C.G. Faber; prof.dr. N.C. Notermans, UU;Dr. I.S.J. Merkies, ius promovendi.

Shenghua Zong. Autoantibodies in disorders of the brain: expanding the spectrum. Supervisor: prof.dr. P. Marinez; co-supervisor: dr. M. Losen; dr. R. Rouhl.

Jan-Willem Kallewaard. Diagnosis and minimally invasive treatment of chronic discogenic low back pain. Supervisor: prof.dr. M. van Kleef; co-supervisors: prof.dr. H. van Santbrink; dr. P. Willems.

Simone M. Crivelli. Sphingolipid metabolism in the pathophysiology and treatment of Alzheimer's disease. Supervisors: prof.dr. P. Martinez-Martinez; prof.dr. E. de Vries, VUmc. Co-supervisors: dr. M. Losen; dr. M. Mulder, Rotterdam.

Natasha Pahuja. Etiopathogenesis, advanced imaging and treatment outcomes in Asian Indians with keratoconus. Supervisor: prof.dr. R. Nuijts, co-supervisor: dr. R. Shetty, Bengaluru.

Pooja Khamar Mayur Raksha. Clinical, Molecular and Biomechanical outcomes of SMILE (small incision lenticule extraction) and other refractive surgery techniques. Supervisor: prof.dr. R. Nuijts, co-supervisor: dr. R. Shetty, Bengaluru.

Niels Janssen. Patterns and pathways. Indicators for potential improvements of dementia care. Supervisors: prof.dr. F. Verhey; prof.dr.mr. S. Evers; Co-supervisor: dr. R. Handels.

Giovanni Mansueto. Childhood adversities and Psychosis: investigation of the potential aetiopathogenetic mechanisms. Supervisor: prof.dr. K. Schruers; co-supervisors: prof.dr. F. Cosci, University of Florence, It; prof.dr. R. van Winkel, KU Leuven.

Joke Debruyne. Cochlear implantation in adults with early-onset deafness. Supervisors: prof.dr. B. Kremer; prof.dr.ir. T. Francart, KU Leuven; Co-supervisor: dr.ir. J. Brokx.

Koenraad Meuwissen, Burst Spinal Cord Stimulation in a Rat Model of Chronic Neuropathic Pain: Spinal and Supraspinal Mechanisms. Supervisors: prof. dr. E.A.J. Joosten; prof. dr. M. van Kleef.

Lisa Schmiedek, Episodic memory in ageing and AD: a possible target for electrical stimulation ? Supervisors: prof. dr. F.R.J. Verhey; prof. dr. A.T. Sack; co-supervisor: dr. H.I.L. Jacobs 
Paolo Maino, Implantable Intrathecal Drug Delivery in Treatment of Chronic Intractable Pain and Spasticity: Improvement of Safety and the Use of Imaging Techniques. Supervisors: prof. dr. E.A. Joosten; prof. dr. M. van Kleef.

José Geurts, Chronic Pain; Impact of Chronic Pain on a Societal, Personal, and Treatment Level. Supervisors: prof. dr. C.D. Dirksen; prof.dr. M. van Kleef; co-supervisor: dr. P.C. Willems.

Brigitte Brouwer, Painful Small Fiber Neuropathy; Symptoms, assessments and interventions. Supervisor: prof. dr. C.F. Faber; co-supervisors: dr. I.S.J. Merkies, Willemstad, Curaçao; dr. J.G.J. Hoeijmakers.

Ruth Gussenhoven, Antenatal inflammatory insults and preterm brain injury: Pathophysiology and therapeutic strategies. Supervisors: prof. dr. B.W. Kramer; prof. dr. L.J.I. Zimmermann; Dr. T.G.A.M. Wolffs.

Adriana (Janine) Collet, Specific Care on the Interface of Mental health and Nursing home "SpeCIMeN". Supervisors: prof. dr. M.E. de Vugt; prof. dr. J.M.G.A. Schols; Prof. dr. F.R.J. Verhey.

Fares Nigim, Glioblastoma and Meningioma Biology, Targeted Therapy and Oncolytic Virus Therapy. Supervisors: prof. dr. Y. Temel; prof. dr. S.D. Rabkin, Harvard; cosupervisors: dr. H. Wakimoto, Harvard; dr. L. Ackermans.

Leonie Banning, Neuropsychiatric symptoms in Alzheimer's disease; Associations with biomarkers. Supervisor: prof. dr. F.R.J. Verhey; co-supervisors: dr. P. Aalten; Dr. I.H.G.B. Ramakers.

Johan Haumann, Prevalence and pharmacological treatment of pain in patients with cancer; The role of opioids with and without NMDA receptor affinity. Supervisor: prof.dr. E.A. Joosten; cosupervisors: Prof.dr. M.H.J. van den Beuken-van Everdingen; dr. S.M.J. Van Kuijk.

Joost Riphagen, Vascular matters in aging and dementia. Supervisor: prof.dr. F.R.J. Verhey; cosupervisor: Dr. H.I.L. Jacobs.

\section{0}

Nikos Priovoulos, Structural and functional imaging of the locus coeruleus at $7 \mathrm{~T}$ : from methodological to clinical application. Supervisor: prof.dr. F.R.J. Verhey; co-supervisors: Dr. H.I.L. Jacobs; dr. B.A. Poser.

Simone Verhagen, The power of individual landscapes; A clinical exploration of personal experience sampling and new horizons. Supervisors: prof.dr. P.A.E.G. Delespaul; prof.dr. J.J. van Os, UM/UU; co-supervisor: dr. C.J.P. Simons.

Nagy Youssef, Epigenetics, resilience and brain stimulation: advances in the mechanistic and therapeutic utility in patients with affective (PTSD and mood) disorders. Supervisor: Prof.dr. B.P.F. Rutten; co-supervisor: Prof. dr. P. Sienaert, KU Leuven.

Abhishek Appaji, Retinal vascular features as a biomarker for psychiatric disorders. Supervisor: Prof. Dr. C.A.B. Webers; co-supervisor: Dr. T.T.J.M. Berendschot, Dr. Naren P. Rao.

Koos Hovinga, Angiogenesis Inhibition in Glioblastoma. Supervisor: prof. dr. Y. Temel; cosupervisor: Prof. V. Tabar, New York, USA.

Gerhard Drenthen, Myelin and networks, Magnetic Resonance Imaging in Epilepsy. Supervisors: prof.dr.ir. W.H. Backes; Prof.dr. A.P. Aldenkamp; co-supervisor: dr. J.F.A. Jansen.

Anna Gorlova, Understanding the Molecular Mechanisms of Aggression in BALB/C and TPH2Deficient Mice. Supervisor: prof.dr. K. Lesch, Universitätsklinikum Würzburg, cosupervisors: dr. T. Strekalova; prof.dr. L. Bettendorff, University of Liège.

Ekaterina Veniaminova, The impact of the 'Western Diet' on Emotional, Social and Cognitive Behaviours as revealed by a study on conventional and serotonin Transporter-Deficient Mice. 
Supervisor: prof.dr. K. Lesch, Universitätsklinikum Würzburg, co-supervisors: dr. T. Strekalova; prof. D.C. Anthony, Oxford.

Dmitrii Pavlov, The contribution of CNS inflammation and Glycogen Synthase Kinase-3 (GSK-3)cascades on adverse memory learning on mouse models of emotional stress. Supervisor: prof.dr. K. Lesch, Universitätsklinikum Würzburg, co-supervisors: dr. T. Strekalova; prof.dr. L. Bettendorff, University of Liège.

Eric Fonseca Wald, Absence Epilepsy and Panayiotopoulos Synrome: Neurocognition and Brain Development. Supervisor: prof.dr. R.J. Vermeulen; co-supervisors: Dr. S. Klinkenberg; dr. M.J.A. Debeij-van Hall; Dr. J.G.M. Hendriksen, Epilepsiecentrum Kempenhaeghe.

Kimberley S. Noij, Cervical vestibular evoked myogenic potentials; Toward optimizing clinical use. Supervisors: prof.dr. H. Kingma; prof. S.D. Rauch, MD, Massachusetts Eye and Ear, Harvard; cosupervisor: Dr. R. van de Berg.

Mark J. van Tilburg, Advancement in cVEMP's. Supervisors: prof.dr. H. Kingma; prof.dr. S. Rauch, Harvard; co-supervisors: dr. R. van de Berg; dr. B. Herrmann, Boston.

Nalini Atcharayam, Duchenne Muscular Dystrophy: The NIMHANS Experience. Supervisors: prof.dr. T. Delhaas; prof.dr. B.W. Kramer.

Murat L Atagün, Cognitive neurophsysiology and neurochemistry in bipolar disorder. Supervisor: Prof. Dr. Therese van Amelsvoort; co-supervisors: Dr. Sinan Guloksuz; Dr. Marian Drukker.

Majed Aldehri, Deep brain stimulation, memory functions and mechanisms. Supervisor: Prof. dr. Y. Temel; co-supervisors: dr. S. Hescham; dr. A. Jahanshahianvar. 